Old Dominion University

ODU Digital Commons

Physics Theses \& Dissertations

Physics

Winter 2018

\title{
Crab Cavity Requirements for the Jefferson Lab Electron-Ion Collider
}

Salvador Isaac Sosa Güitrón

Old Dominion University

Follow this and additional works at: https://digitalcommons.odu.edu/physics_etds

Part of the Elementary Particles and Fields and String Theory Commons, and the Nuclear Commons

\section{Recommended Citation}

Sosa Güitrón, Salvador I.. "Crab Cavity Requirements for the Jefferson Lab Electron-Ion Collider" (2018). Doctor of Philosophy (PhD), Dissertation, Physics, Old Dominion University, DOI: 10.25777/z0kq-ms51 https://digitalcommons.odu.edu/physics_etds/18

This Dissertation is brought to you for free and open access by the Physics at ODU Digital Commons. It has been accepted for inclusion in Physics Theses \& Dissertations by an authorized administrator of ODU Digital Commons. For more information, please contact digitalcommons@odu.edu. 


\title{
CRAB CAVITY REQUIREMENTS FOR THE JEFFERSON LAB ELECTRON-ION COLLIDER
}

\author{
by \\ Salvador Isaac Sosa Güitrón \\ B.S. February 2012, Benemérita Universidad Autónoma de Puebla, Mexico. \\ M.S. May 2014, Old Dominion University
}

\begin{abstract}
A Dissertation Submitted to the Faculty of
Old Dominion University in Partial Fulfillment of the

Requirements for the Degree of

DOCTOR OF PHILOSOPHY

PHYSICS

OLD DOMINION UNIVERSITY
\end{abstract}

December 2018

Approved by:

Jean R. Delayen (Director)

Geoffrey Krafft (Member)

Todd Satogata (Member)

Mark Havey (Member)

Mohammad Zubair (Member) 


\title{
ABSTRACT \\ CRAB CAVITY REQUIREMENTS FOR THE JEFFERSON LAB ELECTRON-ION COLLIDER
}

\author{
Salvador Isaac Sosa Güitrón \\ Old Dominion University, 2018 \\ Director: Dr. Jean R. Delayen
}

An Electron-Ion Collider (EIC) has been proposed to be the next large facility to explore the dynamics of quarks and gluons inside nuclei. A conceptual design for a Jefferson Lab Electron-Ion Collider (JLEIC) is being developed. It is based on the CEBAF 12 GeV machine as a full energy electron beam injector. Two crucial requirements of JLEIC are high luminosity in the $10^{34} \mathrm{~cm}^{-2} \mathrm{~s}^{-1}$ range, and full acceptance detection of particles. The full acceptance requirement relies on having the beams collide at a relatively large crossing angle of $50 \mathrm{mrad}$. Such a large crossing angle, however, can reduce the luminosity by as much as an order of magnitude. One way to compensate for geometric luminosity loss due to the beam crossing angle is using crab cavities. Crab cavities are superconducting radio-frequency structures optimized to produce a transverse force that "tilts" the beam bunches in such a way that a head-on collision is restored at the collision point, thus restoring the luminosity. A second set of crab cavities is placed downstream of the collision point and is used to cancel the initial bunch tilt, ideally suppressing any crab-induced effects anywhere else in the rings. In this dissertation, we study the dynamics of JLEIC beams with a bunch crabbing scheme using particle tracking simulations. We first evaluate the beam stability when crab cavities are included in the collider rings and then use this stable model to study effects of crab cavity multipoles and RF noise. We also study the role of crab cavity higher-order modes (HOM) in driving coupled-bunch instability and identify dangerous HOM which need to be damped. A specific crab cavity for JLEIC has not yet been selected. It is the purpose of this dissertation to provide general requirements and tolerances to make future crab cavity designs compatible with JLEIC. 
Copyright, 2018, by Salvador Isaac Sosa Güitrón, All Rights Reserved. 


\section{ACKNOWLEDGEMENTS}

I would like first to thank my $\mathrm{PhD}$ advisor, Dr. Jean R. Delayen, for suggesting I work in this project, and specially for his patience and financial support during my time at CAS. Similarly, I'm very grateful to the members of my committee: Dr. Geoffrey Krafft, Dr. Todd Satogata, Dr. Mark Havey and Dr. Mohammad Zubair, for their time in reading this dissertation, for their valuable comments and feedback.

My entire appreciation goes to Dr. Vasiliy Morozov, for accepting me in CASA and guiding me through the beam dynamics and use of accelerator codes used in this work, for his invaluable help in the developing of this dissertation and many hours of discussion. Similarly, to Dr. Fanglei Lin and Dr. Rui Li, for their direct input and feedback in the development of this work, and to all members of CASA for valuable comments. I'm also very grateful to Dr. Grigory Eremeev for his mentorship during my time at the SRF Institute, he had a lot of patience to teach me experimental physics tools for SRF cavities. I would also like to thank Dr. Carlos Hernández-García and his family, for their unconditional friendship and support. Special recognition to Carlos' efforts to connect Mexican students with the accelerator physics field at Jefferson Lab. To my advisors in Mexico: Dr. Arturo Fernández Tellez and Dr. Daniel Tapia Takaki for always showing their interest and support in my PhD career.

To all my friends in CAS, for sharing more than the office space and hours of fun commuting to JLab. In a very special way to Suba and Hyekyong, for their friendship and for providing very valuable data used in this dissertation. To Junki, Alex, Jay, Sajini, Kevin, Chris and everyone else who has come through CAS doors for their friendship. To Giovanni and Gabriel for being the best roommates and friends in many adventures. To Victoria, Alejandra, Tomás and Esly for all the laughs during cookouts at "Little Mexico". To all my friends back home. To my parents and sisters, for their laughs, love, understanding and support during these years, specially through the most difficult times. To Clio, I'm really proud of all you've accomplished so far, I love you, thank you for your support always.

Finally, I would like to acknowledge the Consejo Nacional de Ciencia y Tecnología (CONACYT) and the Consejo de Ciencia y Tecnología del Estado de Puebla (CONCYTEP)

for financially supporting me from 2012 to 2016 with a "5 de Mayo" doctorate scholarship to study in the United States. 


\section{TABLE OF CONTENTS}

Page

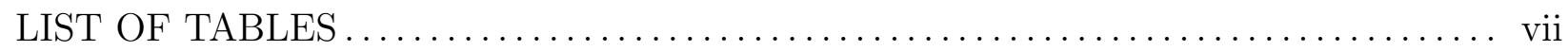

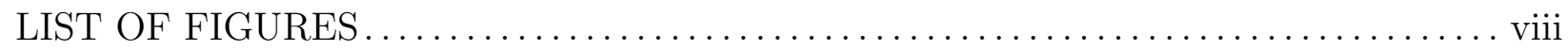

Chapter

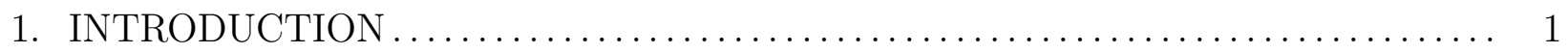

2. JEFFERSON LAB ELECTRON-ION COLLIDER $\ldots \ldots \ldots \ldots \ldots \ldots \ldots \ldots \ldots \ldots$

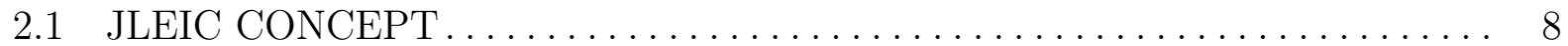

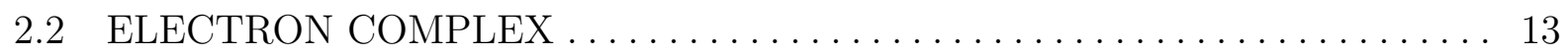

$2.3 \quad$ ION COMPLEX $\ldots \ldots \ldots \ldots \ldots \ldots \ldots \ldots \ldots \ldots \ldots \ldots \ldots \ldots \ldots \ldots$

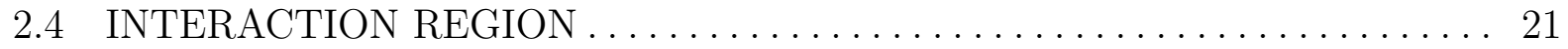

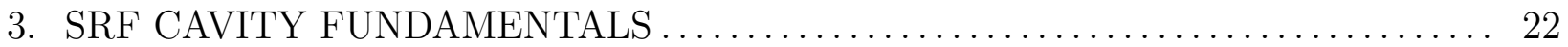

3.1 ELECTROMAGNETIC WAVES IN CAVITIES $\ldots \ldots \ldots \ldots \ldots \ldots \ldots \ldots \ldots 22$

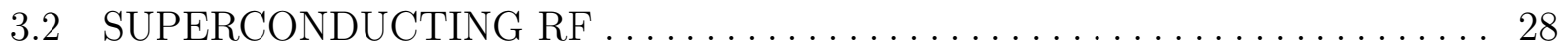

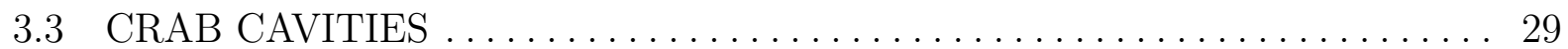

4. BEAM DYNAMICS AND CRAB CROSSING $\ldots \ldots \ldots \ldots \ldots \ldots \ldots \ldots \ldots \ldots \ldots \ldots$

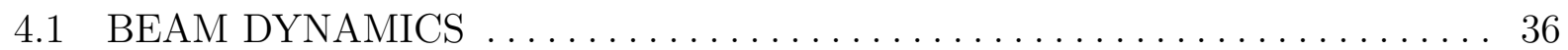

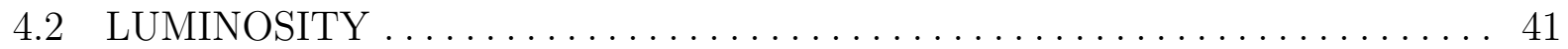

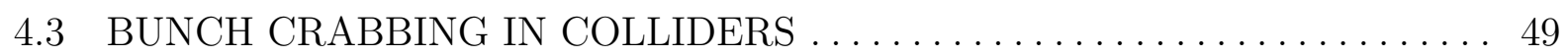

$4.4 \quad$ JLEIC CRAB CROSSING SCHEME $\ldots \ldots \ldots \ldots \ldots \ldots \ldots \ldots \ldots \ldots \ldots \ldots$

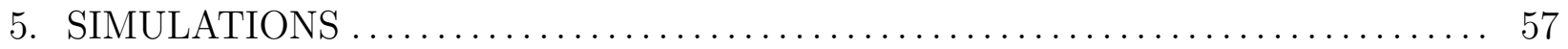

5.1 PARTICLE TRACKING $\ldots \ldots \ldots \ldots \ldots \ldots \ldots \ldots \ldots \ldots \ldots \ldots \ldots \ldots$

5.2 JLEIC MODEL WITH BUNCH CRABBING $\ldots \ldots \ldots \ldots \ldots \ldots \ldots \ldots \ldots \ldots .63$

5.3 CRAB CAVITY MULTIPOLES $\ldots \ldots \ldots \ldots \ldots \ldots \ldots \ldots \ldots \ldots \ldots \ldots \ldots$

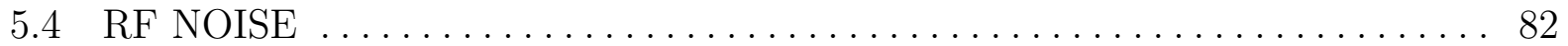

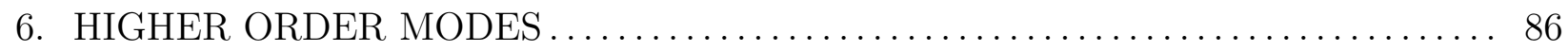

6.1 WAKEFIELD AND IMPEDANCE $\ldots \ldots \ldots \ldots \ldots \ldots \ldots \ldots \ldots \ldots \ldots$

6.2 HIGHER ORDER MODES $\ldots \ldots \ldots \ldots \ldots \ldots \ldots \ldots \ldots \ldots \ldots \ldots \ldots \ldots$

6.3 COUPLED BUNCH INSTABILITY $\ldots \ldots \ldots \ldots \ldots \ldots \ldots \ldots \ldots \ldots \ldots$

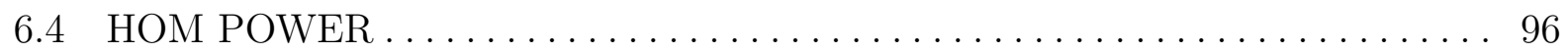

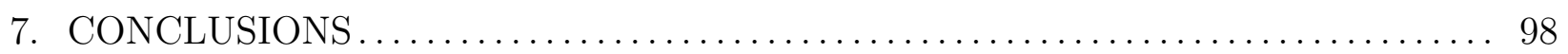

$7.1 \quad$ CRAB CAVITY REQUIREMENTS $\ldots \ldots \ldots \ldots \ldots \ldots \ldots \ldots \ldots \ldots \ldots$

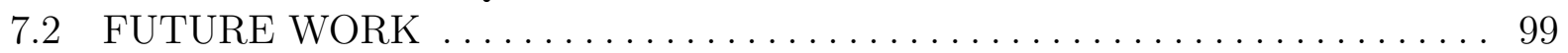




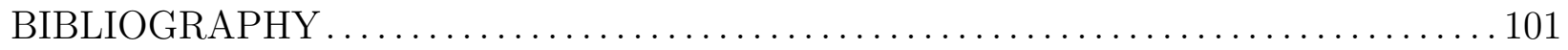
APPENDICES

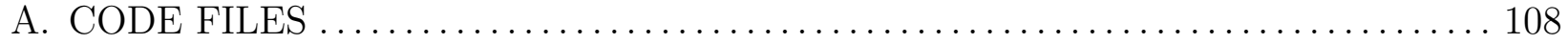
A.1 ELEGANT FILE TO GENERATE A CRABBED BUNCH. . . . . . . . . . . . . 108 A.2 ELEGANT CONFIGURATION FILE TO TRACK A CRABBED BUNCH IN

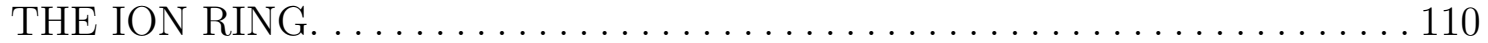
A.3 ELEGANT FILE TO CALCULATE THE DYNAMIC APERTURE. . . . . . . . . 111 A.4 PYTHON SCRIPT FOR LONGITUDINAL CBI CALCULATION. . . . . . . . . . 112

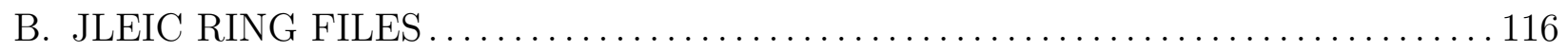

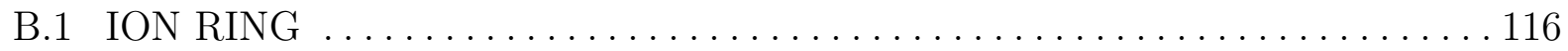

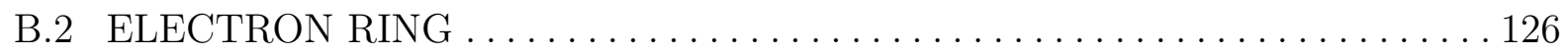

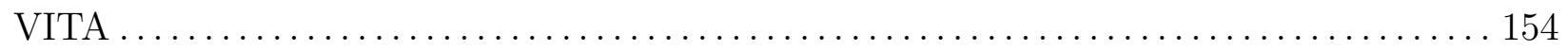




\section{LIST OF TABLES}

Table

Page

1 Parameters of JLEIC in three relevant center-of-mass energy ranges and the ex-

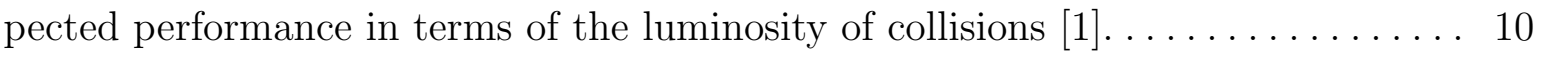

$2 \quad$ Superconducting materials of interest for $\mathrm{rf}$ applications. . . . . . . . . . . . . 29

$3 \quad$ Parameters used for crabbing of electron and positron bunches in KEKB [2]. . . . 32

$4 \quad$ Luminosity reduction factor, $R_{\Theta}$, of different particle colliders [3]. . . . . . . . . 48

5 Bunch crabbing parameters of interest for both electron and proton beams in

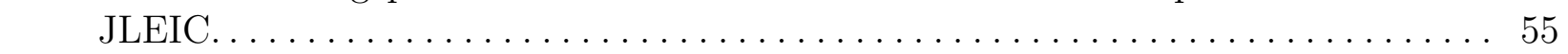

$6 \quad$ Adjusted crab cavity voltage due to a lower $\beta_{\text {crab }}^{x}$ in the baseline ion ring. . . . . . 63

$7 \quad$ Normalized multipole coefficients for 1-cell, 3-cell and squashed elliptical crab cavities. The last row shows the resulting dynamic aperture. $\ldots \ldots \ldots \ldots \ldots \ldots .79$

$8 \quad$ Normalized multipole coefficients that reduce the horizontal DA to $\pm 50 \sigma_{x} . . . .80$

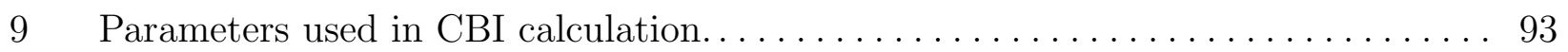

$10 \quad$ Parameters used to estimate the threshold impedance assuming the state-of-art

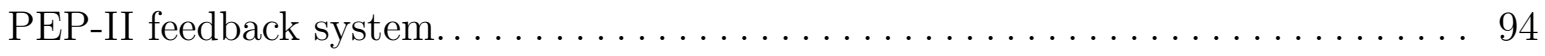

11 Beam spectrum frequencies matched to crab cavity HOMs. Top (bottom) values

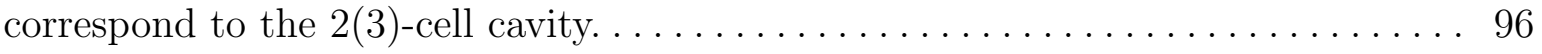

12 Crabbing parameters of the ion ring with a higher betatron function value $\beta_{x}=$

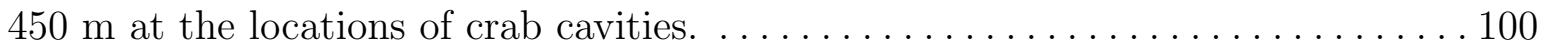

13 Crabbing parameters of the electron and ion rings with $476.3 \mathrm{MHz}$ crab cavities. 100 


\section{LIST OF FIGURES}

Figure

1 Concept of eRHIC at Brookhaven National Lab. An electron ring will be added

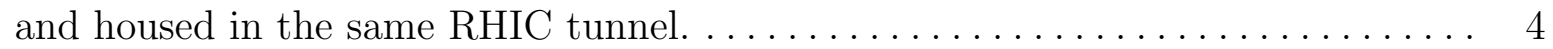

2 Concept of EIC at Jefferson Lab with CEBAF $12 \mathrm{GeV}$ as an electron beam injector. Unique figure-8 rings will be added and are designed to achieve high-

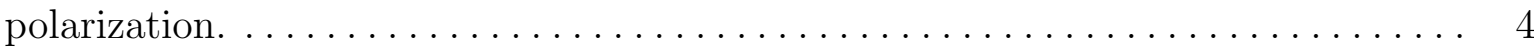

3 QCD Physics enabled by a high luminosity EIC. New frontiers are enabled with the baseline concept of JLEIC. $\ldots \ldots \ldots \ldots \ldots \ldots \ldots \ldots \ldots \ldots \ldots \ldots \ldots \ldots \ldots \ldots \ldots \ldots \ldots \ldots \ldots$

$4 \quad$ First crabbing of a proton bunch at the Super Proton Synchrotron (SPS) accel-

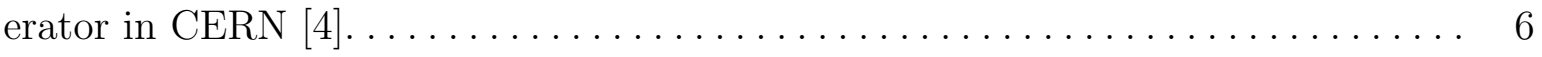

5 Aerial view of the Jefferson Lab site. It shows the proposed facilities to house the $\sim 2.5 \mathrm{~km}$ figure- 8 rings of JLEIC. $\ldots \ldots \ldots \ldots \ldots \ldots \ldots \ldots \ldots$

$6 \quad$ Expected luminosity of JLEIC as a function of the center-of-mass energy. Different curves correspond to different beam bending (dipole) magnets [1]. . . .... 12

7 Diagram of the Continuous Electron Beam Accelerator Facility (CEBAF) at Jefferson Lab and the elements added for the $12 \mathrm{GeV}$ upgrade. . . . . . . . . . 14

8 A $500 \mathrm{MHz}$ RF separator cavity is used at CEBAF to separate and send the

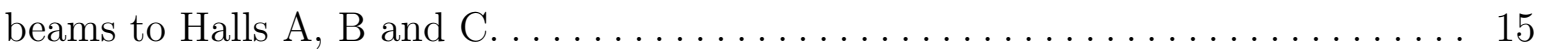

9 Diagram of the beam path and its energy in CEBAF $12 \mathrm{GeV}$, from the electron gun to the experimental Halls $\mathrm{A}, \mathrm{B}, \mathrm{C}$ and $\mathrm{D} . \ldots \ldots \ldots \ldots \ldots \ldots \ldots$

$10 \quad$ Figure-8 electron collider ring for JLEIC and its main optical elements. . . . . . . 17

11 Ion source and linac for the first-step acceleration of the ion beam. . . . . . . . 18

12 Footprint of the ion booster ring and its corresponding optics. . . . . . . . . 18

13 Layout of the ion collider ring, which is matched to the electron ring. This ring has reserved $10 \mathrm{~m}$ of space for crab cavities at each side of the IR. . . . . . . . 19

14 Concept of bunched beam cooler for the high energy ion beam in JLEIC. . . . . . 20

15 Diagram of the interaction region of JLEIC. The ion beam meets the electron ring plane for the collision. The detector is aligned with the electron beam. . . . . . 21 


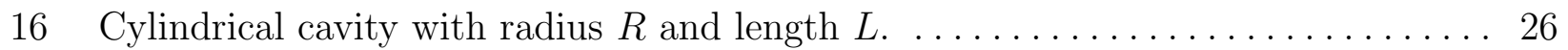

17 In a deflector, the rf phase is such that the maximum transverse kick happens at

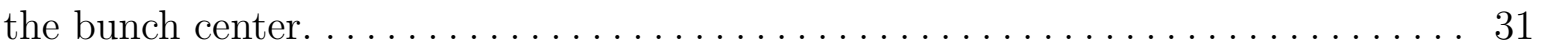

18 In a bunch crabber, the phase is set up such that the field crosses zero at the

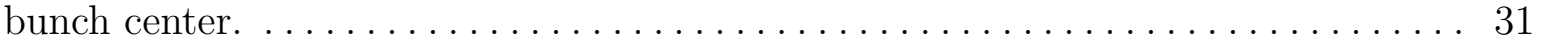

19 Diagram of the squashed elliptical crab cavity used in KEKB. . . . . . . . . . . . 33

20 Crab cavity concepts for the HL-LHC upgrade, DQW and RFD cavities for vertical and horizontal bunch crabbing. $\ldots \ldots \ldots \ldots \ldots \ldots \ldots \ldots \ldots \ldots \ldots \ldots \ldots$

$21 \quad$ Prototype of a $750 \mathrm{MHz}$ rf-dipole crab cavity for MEIC. . . . . . . . . . . . . 34

22 Multi-cell rf-dipole crab cavity concept for JLEIC modeled in CST Microwave Studio [5], the arrows show the electric field lines. $\ldots \ldots \ldots \ldots \ldots \ldots \ldots \ldots \ldots$

23 System of coordinates where $s$ is aligned to the design beam trajectory. . . . . . . 37

24 The beam "paints" an area in phase space, characterized by the Twiss parameters. 40

25 Idealized collision of two cylindric bunches with $n_{1}$ and $n_{2}$ particles and interaction cross section $\sigma_{\text {int }} . \ldots \ldots \ldots \ldots \ldots \ldots \ldots \ldots \ldots \ldots \ldots \ldots \ldots \ldots \ldots \ldots \ldots$

26 Beams crossing in the plane $x z$ with a crossing angle $\alpha_{x}$ and a transverse offset

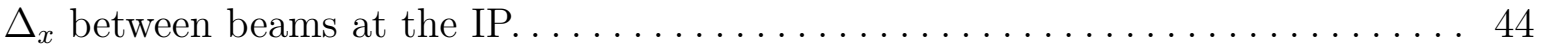

27 Qualitative dependence of the luminosity reduction factor with the Piwinski angle. 48

28 Local bunch crabbing scheme for a symmetric interaction region of an electronion collider. The betatron phase advance between each crab cavity and IP is

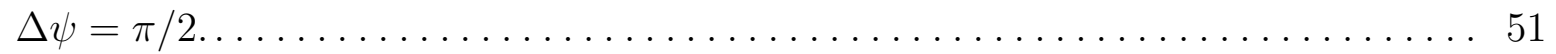

29 Global vs. local bunch crabbing scheme. The local crabbing scheme acts as a

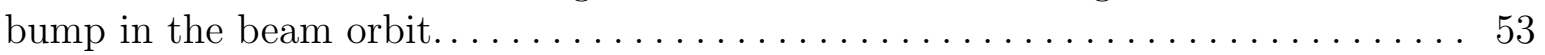

30 Optics of the Chromatic Compensation Blocks of the electron ring [6]. . . . . . . . 54

31 Optical sections of the CCB for the ion ring. This includes $10 \mathrm{~m}$ space reserved for crab cavities at each side of the interaction region $[6] . \ldots \ldots \ldots \ldots \ldots \ldots 4$

32 Injection optics of the ion ring. $\ldots \ldots \ldots \ldots \ldots \ldots \ldots \ldots \ldots \ldots \ldots \ldots \ldots \ldots \ldots$

33 First version of the JLEIC ion ring where local crabbing was implemented, the circumference of the ring is $2153.9 \mathrm{~m} . \ldots \ldots \ldots \ldots \ldots \ldots \ldots \ldots \ldots \ldots \ldots \ldots \ldots \ldots \ldots$ 
34 Optics of the ion ring lattice with the vertical CCB closer to IP. . . . . . . . . . 61

35 Collision optics of the JLEIC lattice with switched horizontal/vertical CCB. . . . . 62

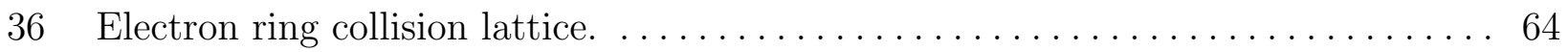

37 Initial distribution of a $100 \mathrm{GeV}$ protons bunch with 10000 particles. . . . . . . . 65

$38 \quad$ Crabbed electron and ion bunches at IP at different energies. . . . . . . . . . 66

$39 \quad$ Crab angle at the IP in the baseline ion ring lattice, Lattice $1 . \ldots \ldots \ldots \ldots 67$

$40 \quad$ Emittance growth due to crab cavities in the baseline ion ring lattice. . . . . . . . 68

41 Crab angle evolution at the IP in the lattice with switched CCB. Angle is shown

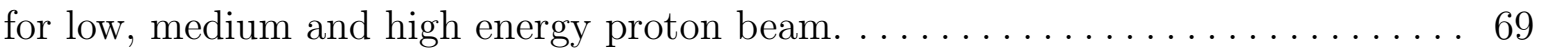

$42 \quad$ Crab angle evolution in the electron ring at $5 \mathrm{GeV} . \ldots \ldots \ldots \ldots \ldots \ldots \ldots \ldots$

43 Correlation of $x z$ coordinate at the location of the first crab cavity. . . . . . . . 70

44 Fractional horizontal and vertical betatron tunes. . . . . . . . . . . . . . . 71

45 The crab cavity voltage is linearly ramped over 1000 turns. . . . . . . . . . . . . 72

46 Projected normalized horizontal beam emittance in the modified lattice. The growth is due to the establishment of a new matched ellipse. The voltage on the crab cavities is ramped over 10, 100, 500, 1000 and 2000 turns for comparison. Emittance can be preserved as long as the turn-on rate is longer than 500 turns. . 73

47 Calculation of the ring dynamic aperture using elegant. $\ldots \ldots \ldots \ldots \ldots \ldots \ldots$

48 Beam DA of the ion ring with the crab cavity voltage set to 0 and $20.82 \mathrm{MV}$ for

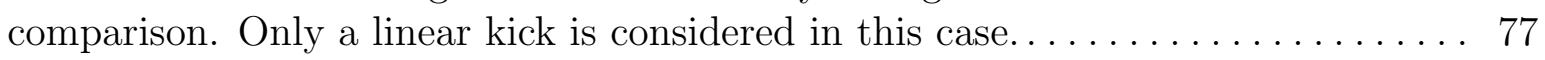

49 Crab cavity concepts for JLEIC used for multipole studies. Shown are single-cell, 3-cell crab and squashed elliptical cavity designs with $60 \mathrm{~mm}$ and $70 \mathrm{~mm}$ apertures. 78

50 Different inner pole geometries used on the single-cell and 3-cell rf-dipole crab cavities. Left figure shows a flat pole tip, right figures shows a curved pole tip. . . . 78

51 The resulting DA corresponding to the crab cavity designs shown in Fig. 49 .. . . . 80

52 Resulting DA from multipole coefficients of single-cell and 3-cell rf-dipole crab cavity concepts with different apertures and pole tip geometries. . . . . . . . . 81

53 Resulting DA from multipole coefficients of squashed cavities with $60 \mathrm{~mm}$ and 70

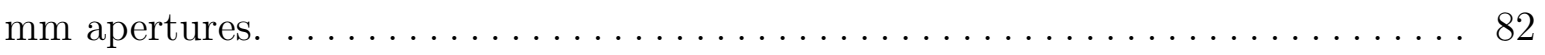


54 An error in the crab cavity voltage amplitude produces an error in the tilt angle

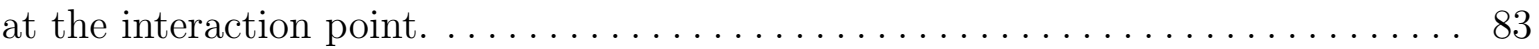

55 Distribution of the crab angle at IP over $5 \times 10^{4}$ turns. Fractional $\mathrm{rms}$ noise $\Delta V / V=0.1 \%, 0.05 \%$ and $0.01 \%$ are assumed on the amplitude of the crab

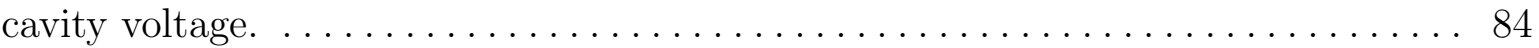

56 Emittance increase due to different noise levels in the amplitude of the crab cavity

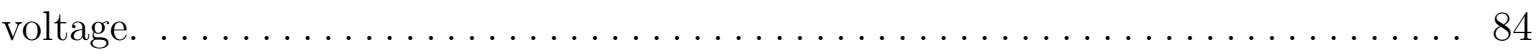

57 A crab cavity voltage phase error, or bunch arrival time error, produce a trans-

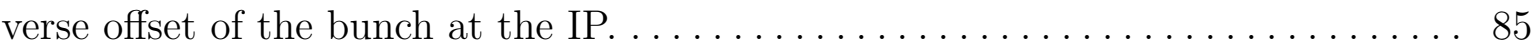

58 Beam emittance as a function of turns for different phase noise levels in the crab

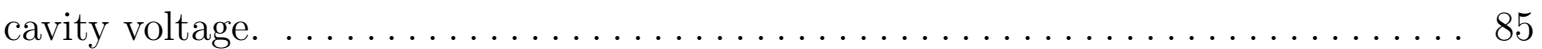

59 Crab cavity concepts with hooks and probes for study of HOMs: 2-cell and 3-cell

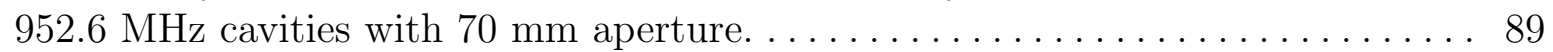

60 Longitudinal HOM impedance from 2 and 3-cell crab cavity rf-dipole designs. The solid line shows the threshold impedance assuming the state-of-art rf feedback system used in PEP-II. $\ldots \ldots \ldots \ldots \ldots \ldots \ldots \ldots \ldots \ldots \ldots \ldots \ldots \ldots$

61 Bunches in a beam can become coupled by a long-ringing wakefield in an rf cavity. 90

62 Diagram of the calculation to identify cavity HOMs driving coupled-bunch oscil-

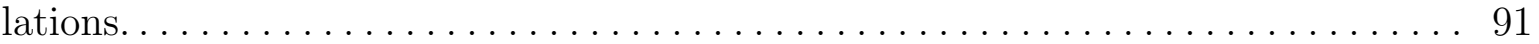

63 Beam spectrum of an uniformly-spaced train of bunches with Gaussian and

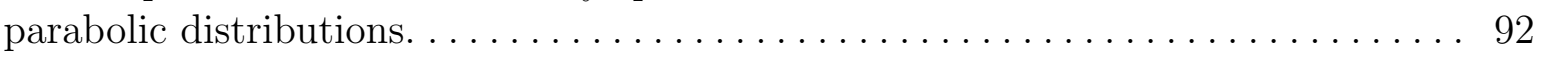

64 Coupled-bunch instability growth rate vs. mode number corresponding to the 2 sets of crab cavity longitudinal HOMs. $\ldots \ldots \ldots \ldots \ldots \ldots \ldots \ldots \ldots \ldots$

65 Power from HOMs estimated for on-resonance excitation case. . . . . . . . . . . . 97 66 Crabbed bunch at IP using $476.3 \mathrm{MHz}$ and $952.6 \mathrm{MHz}$ crabbing parameters. . . . 101 


\section{CHAPTER 1}

\section{INTRODUCTION}

Nuclear science is concerned with the study of atomic nuclei, which practically accounts for all the observed mass in the Universe. After the discovery of protons and neutrons at the beginning of the twentieth century, it was universally agreed that they were the basic components of the nucleus in an atom, even helping explain the chemistry of elements. With the advent of high energy colliders came the Standard Model of particles and fields, in which nucleons (protons and neutrons) are no longer fundamental particles, but rather have internal structure. In the Standard Model, nucleons are formed by fundamental particles called quarks and held together by gluons: the mediating particles of the strong interaction. In this framework, protons are composed of two quarks of the type " $u p$ " and one quark of the type "down", while neutrons are formed of two quarks "down" and one quark "up". In general, a particle that is composed by 2 or 3 quarks is called a hadron.

Quantum Chromodynamics (QCD) is a theory that describes the strong interaction between quarks and gluons inside the nucleon. A fundamental goal of modern nuclear physics is to explain the emergence of nucleons and their properties, such as mass and spin, from the dynamics of quarks and gluons within QCD. One of the main characteristics of matter in QCD is that both quarks and gluons carry a "color" charg€1. Because the gluons carry color charge, the dynamics becomes very rich, as not only do they hold the quarks together, but they can also interact with other gluons.

The mass of individual quarks is significantly smaller than the mass of the composite nucleon, and thus the interaction between gluons must play an important role in the emerging properties of nucleons. However, the regime of matter where gluons dominate the interactions in QCD has not been thoroughly explored. This is mainly because of the technological limitation of available particle colliders. In the Continuous Electron Beam Accelerator Facility (CEBAF) at Jefferson Lab, for example, a high energy electron beam is used to probe the nuclei in stationary targets. The available center of mass energy of the collision with a fixed target, however, scales with the square-root of the beam energy, thus limiting the energy reach of the interactions. In a conventional particle collider like the Large Hadron

\footnotetext{
${ }^{1}$ In contrast, this is a property not present in Quantum Electrodynamics (QED), where the mediating particle of the electromagnetic force, the photon, does not carry electric charge.
} 
Collider (LHC) at CERN, or the Relativistic Heavy Ion Collider (RHIC) at Brookhaven National Lab, the target is itself a high energy beam. Hadron beams are brought into collision with each other and thus the center of mass energy of the collision is higher. This is still not ideal. Take proton-proton $(p p)$ collisions, for example, where the fundamental interactions during the collision process occur among the components of the proton, i.e. each of the proton components carry only a fraction of the total energy of the proton beam. The effective interaction energies of $p p$ collisions are on the order of $10 \%$ of the total center of mass energy; a $14 \mathrm{TeV}$ center of mass energy collision at the LHC can only probe an energy range less than $2 \mathrm{TeV}$.

Electrons on the other hand, are point-like, fundamental particles. In an Electron-Ion Collier (EIC), electron and ion beams are brought into collision. Not only is the entire energy of the electron beam used to probe the high energy nuclei, but the quantum state of the electron is also well understood, making the EIC an ideal machine to study the gluon dominated regime of QCD. An EIC will expand the QCD science programs at Jefferson Lab and BNL.

The Department of Energy's Nuclear Science Advisory Committee (NSAC) oversees the most important nuclear science projects in the U.S. In recent years, these included the CEBAF energy upgrade from $6 \mathrm{GeV}$ to $12 \mathrm{GeV}$, as well as the construction and commissioning of the Facility for Rare Isotope Beams (FRIB) in Michigan. As a part of the long term science plan, the NSAC recognizes the need of an EIC facility as the next step to explore QCD. From the NSAC 2007 Long Range Plan [7]:

An Electron-Ion Collider (EIC) with polarized beams has been embraced by the U.S. nuclear science community as embodying the vision for reaching the next QCD frontier. EIC would provide unique capabilities for the study of QCD well beyond those available at existing facilities worldwide and complementary to those planned for the next generation of accelerators in Europe and Asia.

Similarly, the EIC was recommended as a high priority project in the NSAC 2015 Long Range Plan [8]:

We recommend a high-energy high-luminosity polarized EIC as the highest priority for the new facility construction following the completion of FRIB.

The physics case and general requirements on an EIC are presented in the so-called EIC White Paper [9]. An EIC should be able to shed some light into the most fundamental questions at the frontier of QCD:

- What are the space and momentum distributions of quarks, gluons and their spin inside 
the nucleon?

- How is the emergence of nucleon properties like mass, spin, etc. explained from the quark-gluon and gluon-gluon interactions?

- How is nuclear binding mechanism explained in terms of quark-gluon interactions?

- Study the gluon density at high energy, in the density saturation regime and unravel whether the saturation happens at a definite boundary and if so, what happens to the quark and gluon distributions at this boundary?

An EIC is suitable to tackle these questions for the following reasons: a collider is required to reach the kinetic energy in the gluon-dominated regime, electron beams are needed as high precision electromagnetic interaction probes, and polarized ion beams enable the study of the correlations of quark and gluon distributions with the spin. It is not the purpose of this dissertation to delve any deeper in the fundamentals of QCD, but rather, on the requirements of the scientific instrument that will enable the further exploration of QCD, the EIC. The EIC requirements extend the probing reach of HERA $2^{2}$ [9], a former lepton-ion collider that operated in Germany until 2007 . General EIC requirements stated on the EIC White Paper are:

- Electron and nucleon beams should be highly polarized, at least $70 \%$ polarization.

- Beams of diverse ion species; from protons and deuterons to heavier Uranium or lead.

- Variable center of mass energies from around 20 to $100 \mathrm{GeV}$, with room for upgrade to $150 \mathrm{GeV}$.

- High collision luminosity above $10^{34} \mathrm{~cm}^{-2} \mathrm{~s}^{-1}$.

- Possibility of having more than one collision point for multiple detectors.

Two national laboratories have brought forward independent proposals for an EIC. Brookhaven National Laboratory (BNL) is developing a machine called eRHIC [10], which among many upgrades, adds an electron ring to the existing Relativistic Heavy Ion Collider. A diagram of eRHIC is shown in Fig. 1. The Thomas Jefferson National Accelerator Facility (TJNAF, JLab) is developing the concept of an EIC based on CEBAF $12 \mathrm{GeV}$ as a highenergy electron beam injector. This machine is called the Jefferson Lab Electron-Ion Collider

\footnotetext{
${ }^{2}$ In German, Hadron-Elektron Ring Anlage.
} 


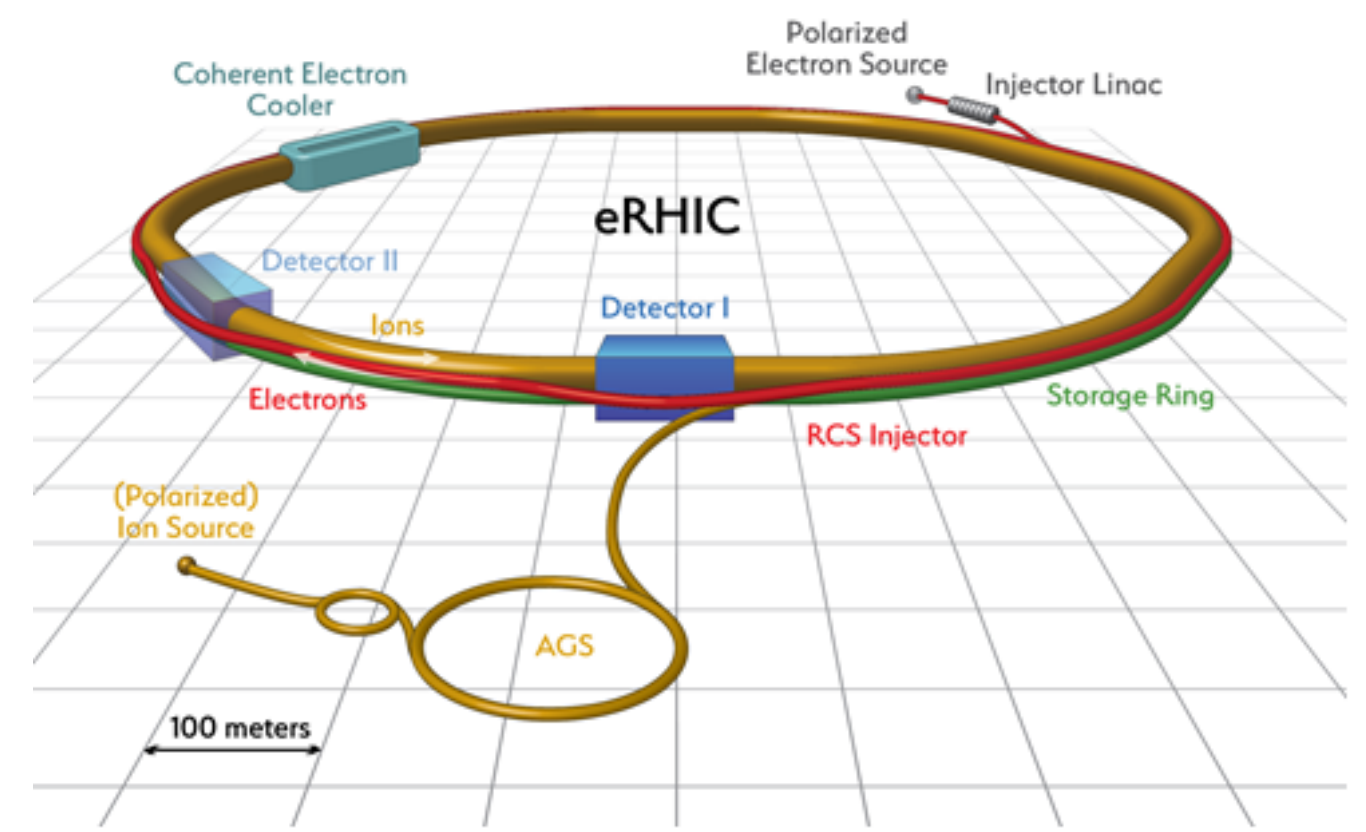

FIG. 1: Concept of eRHIC at Brookhaven National Lab. An electron ring will be added and housed in the same RHIC tunnel.

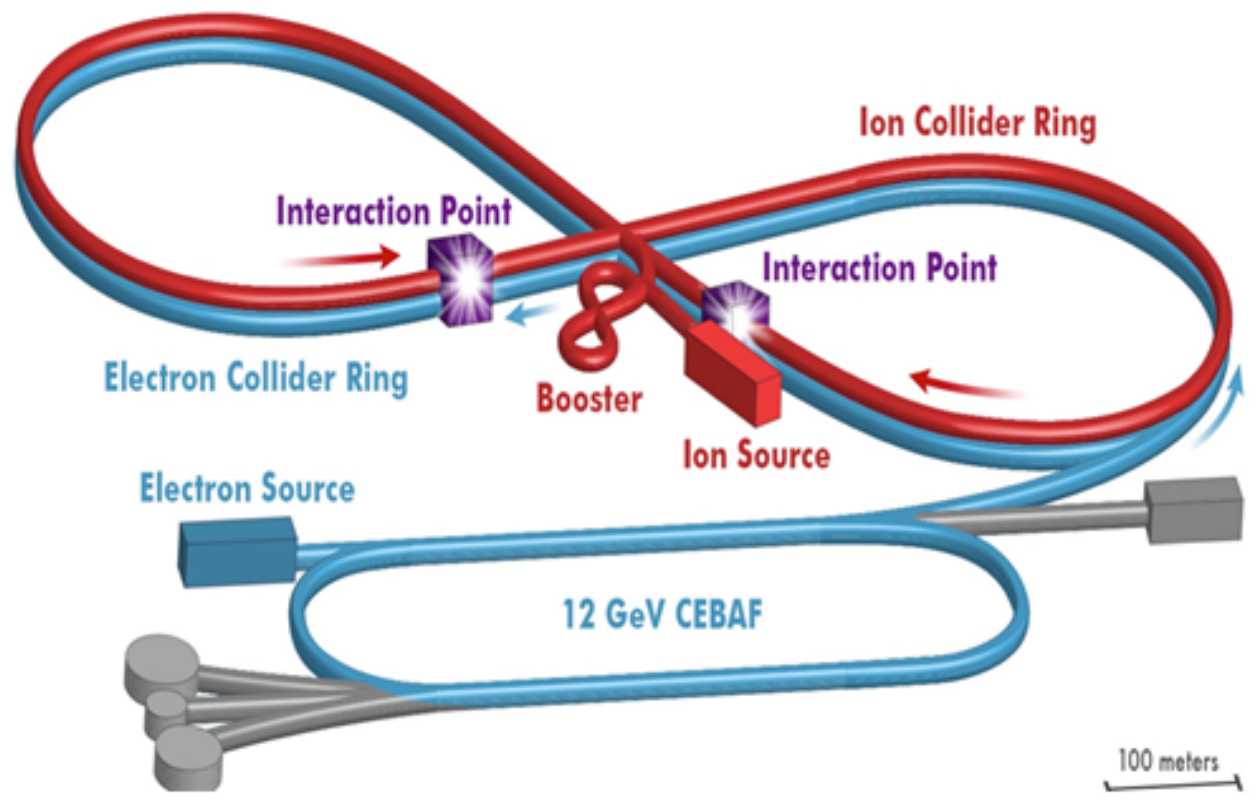

FIG. 2: Concept of EIC at Jefferson Lab with CEBAF $12 \mathrm{GeV}$ as an electron beam injector. Unique figure-8 rings will be added and are designed to achieve high-polarization. 
(JLEIC) [11] and is shown in Fig. 2, Each of these designs comes with its own challenges, as well as common issues that need to be resolved in order to demonstrate technological feasibility and cost effectiveness of the machine. This dissertation represents a specific study within the general Jefferson Lab effort towards the design of JLEIC.

Two of the key requirements on JLEIC are high luminosity collisions and full acceptance detection. Figure 3 shows the relevant QCD physics enabled by the $10^{34} \mathrm{~cm}^{-2} \mathrm{~s}^{-1}$ luminosity of JLEIC. A full acceptance detector, on the other hand, allows for the detection of

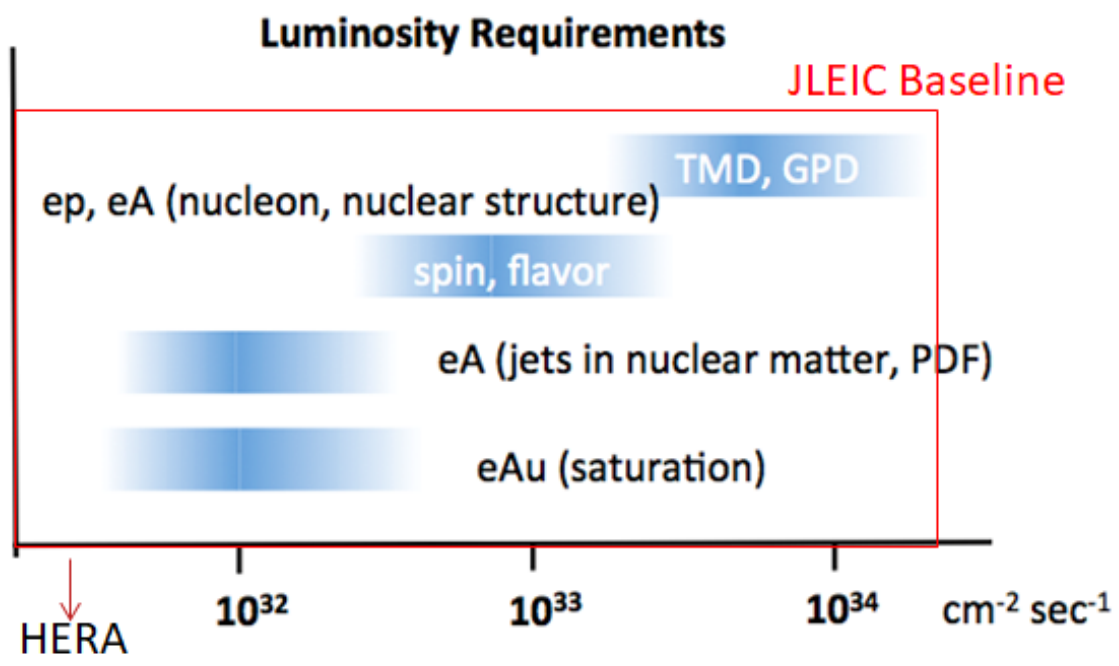

FIG. 3: QCD Physics enabled by a high luminosity EIC. New frontiers are enabled with the baseline concept of JLEIC.

collision products in the forward direction. As it will be discussed in Chapter 4, these two requirements counter each other. The full acceptance detection relies on having the beams collide with a large crossing angle at the interaction point. At the same time, a large beam crossing angle can drastically reduce the collider luminosity. This dissertation concerns the bunch crabbing mechanism that allows preservation of the high luminosity of collisions while beams cross each other at a large crossing angle. The concept of bunch crabbing was first proposed by R. Palmer [12] for linear colliders and soon after by K. Oide and K. Yokoya 13. for circular colliders. In a nutshell, a tilt is induced in both electron and ion bunches previous to the collision such that a head-on collision scenario is recovered at the interaction point, therefore suppressing the geometric loss of luminosity due to the crossing angle.

The crabbing of bunches can be achieved through the use of specially designed superconducting radio-frequency (SRF) structures known as crab cavities. Crab cavities are optimized 
to work on a pure transverse electric (TE) mode, with the phase adjusted such that the electric field is zero at the center of the bunch but is transverse and opposite in the head and the tail of the bunch, thus producing a tilt. The technology of SRF crab cavities was first implemented in 2008, for physics beam runs at the KEKB collider in Japan. KEKB was a $B$ meson factory and implemented squashed elliptical crab cavities for the crabbing of electron beams. The High Luminosity LHC (HL-LHC) upgrade envisions vertical beam crabbing for the ATLAS experiment and horizontal crabbing for the CMS experiment [14]. Two different crab cavities are currently being developed for the HL-LHC project: a double quarter-wave (DQW) crab cavity for ATLAS and rf-dipole (RFD) crab cavity for CMS. At the beginning of 2018, a dedicated test using two DQW prototype cavities at the Super Proton Synchrotron (SPS) in CERN demonstrated for the first time the crabbing of a single $26 \mathrm{GeV}$ proton bunch [4]. Figure 4 shows the tilt in the proton bunch from a head-tail monitor due to a $1 \mathrm{MV}$ crabbing voltage.

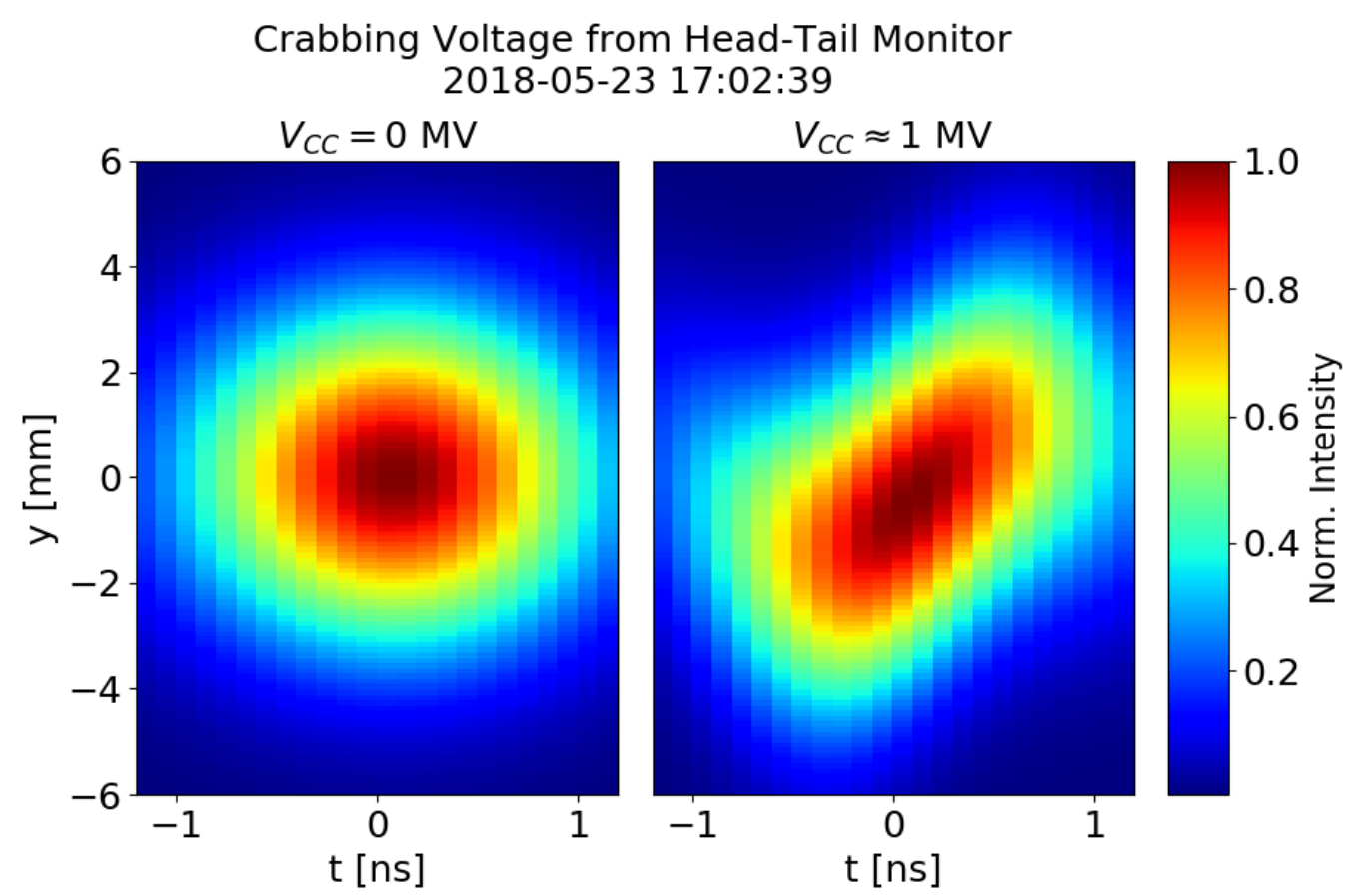

FIG. 4: First crabbing of a proton bunch at the Super Proton Synchrotron (SPS) accelerator in CERN [4.

Similar tests using the rf-dipole prototype cavity are planned for the near future. In Jefferson Lab, the development of the crab cavity systems for JLEIC is a priority, and it is the purpose of this dissertation to support these efforts in the form of accompanying beam dynamics studies. 


\subsubsection{STRUCTURE OF THIS DISSERTATION}

This dissertation is organized in the following way: Chapter 2 describes the main components and unique characteristics of JLEIC existing and proposed facilities, as well as critical technologies being developed. It is intended to sketch the current status of the project as well as to point where this dissertation fits within the JLEIC development effort. Chapter 3 serves as a review of the SRF science, technology and its application to particle accelerators, with the objective to identify the relevant parameters of a crab cavity to be investigated in following chapters. Chapter 4 discusses the beam dynamics in JLEIC rings and is divided in three major sections. In Section 4.1, we introduce general concepts of beam dynamics and specific concepts relevant to the crab crossing scheme, in Section 4.2 we discuss the concept of luminosity in a collider and how it is degraded by a finite crossing angle; in Section 4.3, the general concept of bunch crabbing in a collider is discussed, together with the particulars of a bunch crabbing scheme that is compatible with JLEIC. In Chapter 5, we use the simulation model of JLEIC rings and add the bunch crabbing scheme described in Chapter 4. We then proceed to describe several particle tracking studies designed to get an insight on the beam dynamics induced by crab cavities. With the design of a crab cavity for JLEIC in mind, we study the effects of cavity multipoles and rf field noise on the beam, from which we can determine acceptable multipoles and tolerable noise levels to preserve beam stability in JLEIC. In Chapter 6, the focus is on the instabilities driven by crab cavity

higher order modes (HOM), where we address the identification of dangerous cavity HOM and recommend appropriate HOM damping levels. Finally in Chapter 7 we summarize specific requirements and tolerances on a general crab cavity to make it compatible with JLEIC rings, identify potential issues to be addressed and discuss relevant future studies. 


\section{CHAPTER 2}

\section{JEFFERSON LAB ELECTRON-ION COLLIDER}

The Jefferson Lab Electron-Ion Collider (JLEIC) is designed to satisfy the QCD physics requirements presented in the EIC White Paper [9]. JLEIC is a ring-ring collider concept based on the CEBAF $12 \mathrm{GeV}$ machine as a high energy electron beam injector. This Chapter will describe the major components of JLEIC and it is intended to show where this dissertation fits within the general R\&D effort towards JLEIC. The chapter is arranged in the following way: Section 2.1 discusses the main parameters and characteristics of JLEIC. Section 2.2 describes the electron beam facilities: the existing CEBAF $12 \mathrm{GeV}$ and the electron ring. In a similar way, Section 2.3 describes the ion beam facilities: ion linac, booster and colliding ring. Finally in Section 2.4 we describe the interaction region of the collider, where the detector, and the crab cavities, will be located. The development of JLEIC is a very active and a constantly evolving project. For the purposes of this dissertation, the information contained in this chapter concerns the JLEIC status at the time of the JLEIC Collaboration Meeting in Spring 2018. A more comprehensive and up-to-date description of JLEIC will be available later in the form of a JLEIC pre-Conceptual Design Report (pCDR).

\subsection{JLEIC CONCEPT}

The study of an Electron-Ion Collider (EIC) machine in Jefferson Lab as a future upgrade to CEBAF has been ongoing for many years. Initially, it was conceptualized as an Energy Recovery Linac (ERL) and ring scheme [15], but in 2006, a ring-ring concept was adopted on the grounds of luminosity optimization and minimization of technical risks. This machine was known as the Medium Energy Electron-Ion Collider. For the 2015 MEIC Design Report [11, [16], a considerable change was made by changing the length of the rings. This modification on the length of the rings changed the frequency of the machine from 750 to $476.3 \mathrm{MHz}$, which is now known as JLEIC. This simple but significant modification allowed JLEIC re-use components from the PEP-I] High Energy Ring (HER) [11] in the JLEIC electron ring. By reusing components such as magnets, vacuum chambers and radio-frequency (RF) cavities, both the technical risks and machine cost were reduced. The length increase also allowed

\footnotetext{
${ }^{1}$ PEP-II was an electron-positron collider at SLAC. It was formed by a high-energy electron ring and a low-energy positron ring.
} 
the ion ring to now be based on super-ferric magnet technology, enabling higher energy ion beams.

The general concept of JLEIC is based on the recently upgraded CEBAF $12 \mathrm{GeV}$ machine as a full energy electron beam injector. The EIC must provide a center-of-mass energy in the range from 15 to $65 \mathrm{GeV}$. Since the electron beam energy is essentially locked to the output energy of CEBAF as is, the JLEIC ion ring must provide the remaining energy. The ion ring must be capable of accelerating proton beams up to $100 \mathrm{GeV}$, or in general, ion beams up to $40 \mathrm{GeV}$ per nucleon. Ion beams of interest include polarized light ions, such as protons, deuterons and Helium-3 $\left({ }^{3} \mathrm{He}\right)$, as well as unpolarized heavy ions, up to the element Lead $(\mathrm{Pb})$. JLEIC is formed by two collider rings that are stacked one on top of the other and are housed inside the same tunnel. This machine fits within the Jefferson Lab site, as depicted in the artist's conception in Fig. 5.

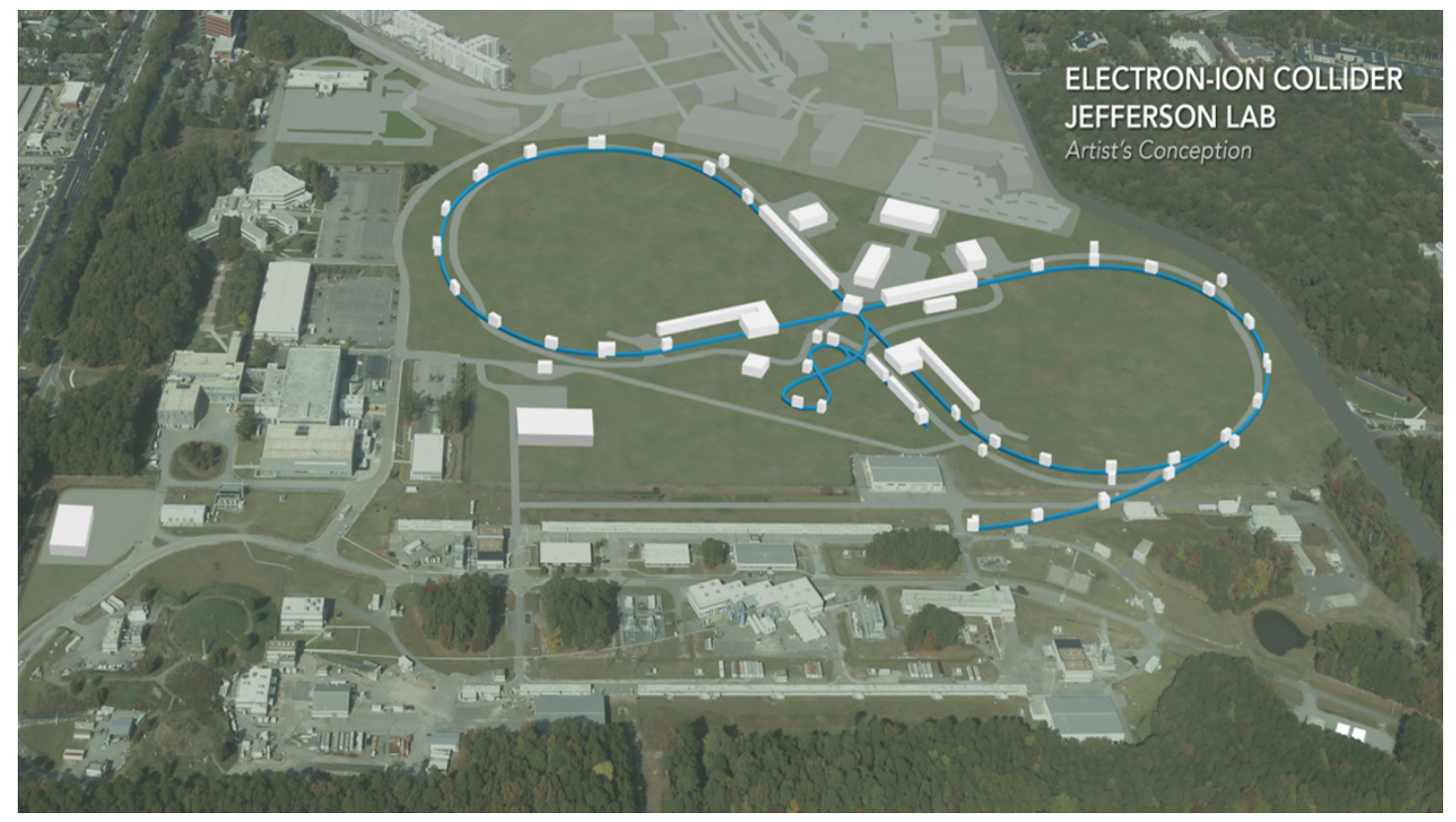

FIG. 5: Aerial view of the Jefferson Lab site. It shows the proposed facilities to house the $\sim 2.5 \mathrm{~km}$ figure-8 rings of JLEIC.

Both collider rings have a unique figure- 8 shape formed by two arcs and two connecting straight sections that cross each other forming a $69^{\circ}$ angle. The arcs are formed by the beam bending magnets and FODO optics. The straight sections have the RF accelerating cavities, the bunched beam cooler, the injection/ejection kickers, the interaction region and space for a second detector to be added in a future upgrade. Table 1 shows general JLEIC parameters and the corresponding performance in terms of the expected luminosity of collisions corresponding to three relevant center-of-mass collision energies [1]. 
TABLE 1: Parameters of JLEIC in three relevant center-of-mass energy ranges and the expected performance in terms of the luminosity of collisions [1].

\begin{tabular}{|c|c|c|c|c|c|c|c|}
\hline Center-of-mass energy & $\mathrm{GeV}$ & \multicolumn{2}{|c|}{21.9 (low) } & \multicolumn{2}{|c|}{44.7 (medium) } & \multicolumn{2}{|c|}{63.3 (high) } \\
\hline Beam type & - & $\mathrm{p}$ & $\mathrm{e}$ & $\mathrm{p}$ & $\mathrm{e}$ & $\mathrm{p}$ & $\mathrm{e}$ \\
\hline Beam energy & $\mathrm{GeV}$ & 40 & 3 & 100 & 5 & 100 & 10 \\
\hline Collision frequency & $\mathrm{MHz}$ & \multicolumn{2}{|c|}{476} & \multicolumn{2}{|c|}{476} & \multicolumn{2}{|c|}{$476 / 4=119$} \\
\hline Particles per bunch & $10^{10}$ & 0.98 & 3.7 & 0.98 & 3.7 & 3.9 & 3.7 \\
\hline Beam current & $\mathrm{A}$ & 0.75 & 2.8 & 0.75 & 2.8 & 0.75 & 0.71 \\
\hline Polarization & $\%$ & 80 & 80 & 80 & 80 & 80 & 75 \\
\hline Bunch length, rms & $\mathrm{cm}$ & 3 & 1 & 1 & 1 & 2.2 & 1 \\
\hline Normalized emittance, hor./vert. & $\mu \mathrm{m}$ & $0.3 / 0.3$ & $24 / 24$ & $0.5 / 0.1$ & $54 / 10.8$ & $0.9 / 0.18$ & $432,86.4$ \\
\hline Horizontal and vertical $\beta^{*}$ & $\mathrm{~cm}$ & $8 / 8$ & $13.5 / 13.5$ & $6 / 1.2$ & $5.1 / 1$ & $10.5 / 2.1$ & $4 / 0.8$ \\
\hline Vert. beam-beam parameter & - & 0.015 & 0.092 & 0.015 & 0.068 & 0.008 & 0.034 \\
\hline Laslett tune-shift & - & 0.06 & $7 \times 10^{-4}$ & 0.055 & $6 \times 10^{-4}$ & 0.056 & $7 \times 10^{-5}$ \\
\hline Detector space, up/down & $\mathrm{m}$ & $3.6 / 7$ & $3.2 / 3$ & $3.6 / 7$ & $3.2 / 3$ & $3.6 / 7$ & $3.2 / 3$ \\
\hline Hourglass (HG) reduction & - & \multicolumn{2}{|r|}{1} & \multicolumn{2}{|c|}{0.87} & \multicolumn{2}{|c|}{0.75} \\
\hline Luminosity per IP, w/HG & $10^{33} \mathrm{~cm}^{-2} \mathrm{~s}^{-1}$ & \multicolumn{2}{|c|}{2.5} & \multicolumn{2}{|c|}{21.4} & \multicolumn{2}{|c|}{5.9} \\
\hline
\end{tabular}


The collider is subject to many single-bunch and multi-bunch instabilities that depend on the beam energy. In the low-energy range, the main limitation is the space charge of the low energy beam. In the medium-energy range, the main limitation to the machine is the strong beam-beam effect. In the high-energy, it is the synchrotron radiation of the electron beam, which needs to be scaled down in order to avoid significant radiation loading.

\section{Future upgrades}

The JLEIC collider is designed to have room for significant future upgrades. For example, depending on the magnet technology used, the ion ring could be upgraded to store higherenergy beams: up to $250 \mathrm{GeV}$ proton beams, or ion beams with $100 \mathrm{GeV}$ per nucleon. Also, the current JLEIC configuration has enough space for two high-luminosity, full-acceptance detectors, one in each of the straight sections, although only one interaction region and detector is being designed for the JLEIC starting configuration. Further upgrades could see JLEIC provide positron-ion collisions, although this is not considered in the baseline design.

\subsubsection{LUMINOSITY}

One of the critical goals of JLEIC is to provide high-luminosity collisions on the order of $10^{34} \mathrm{~cm}^{-2} \mathrm{~s}^{-1}$. To put this number in context, the record for highest luminosity is $2.108 \times 10^{34}$

$\mathrm{cm}^{-2} \mathrm{~s}^{-1}$ [2], and was achieved on June 2009 at the KEKB collider in Japan. This highluminosity requirement is a technological challenge and represents a significant $\mathrm{R} \& \mathrm{D}$ effort that relies on the development of two critical technologies: bunched beam electron cooling for the high-energy ion beam and the use of crab cavities in both electron and ion rings. Figure 6 shows the luminosity goal of JLEIC as a function of the center-of-mass energy and assuming different bending magnet fields for comparison.

\section{JLEIC high-luminosity strategy}

The JLEIC strategy to reach high-luminosity can be summarized in the following schemes or technologies:

- Use of short bunch lengths of approximately $1 \mathrm{~cm}$ in both beams.

- Small transverse beam size and small transverse beam emittance.

- High collision frequency.

- Staged electron cooling of the ion beam. 


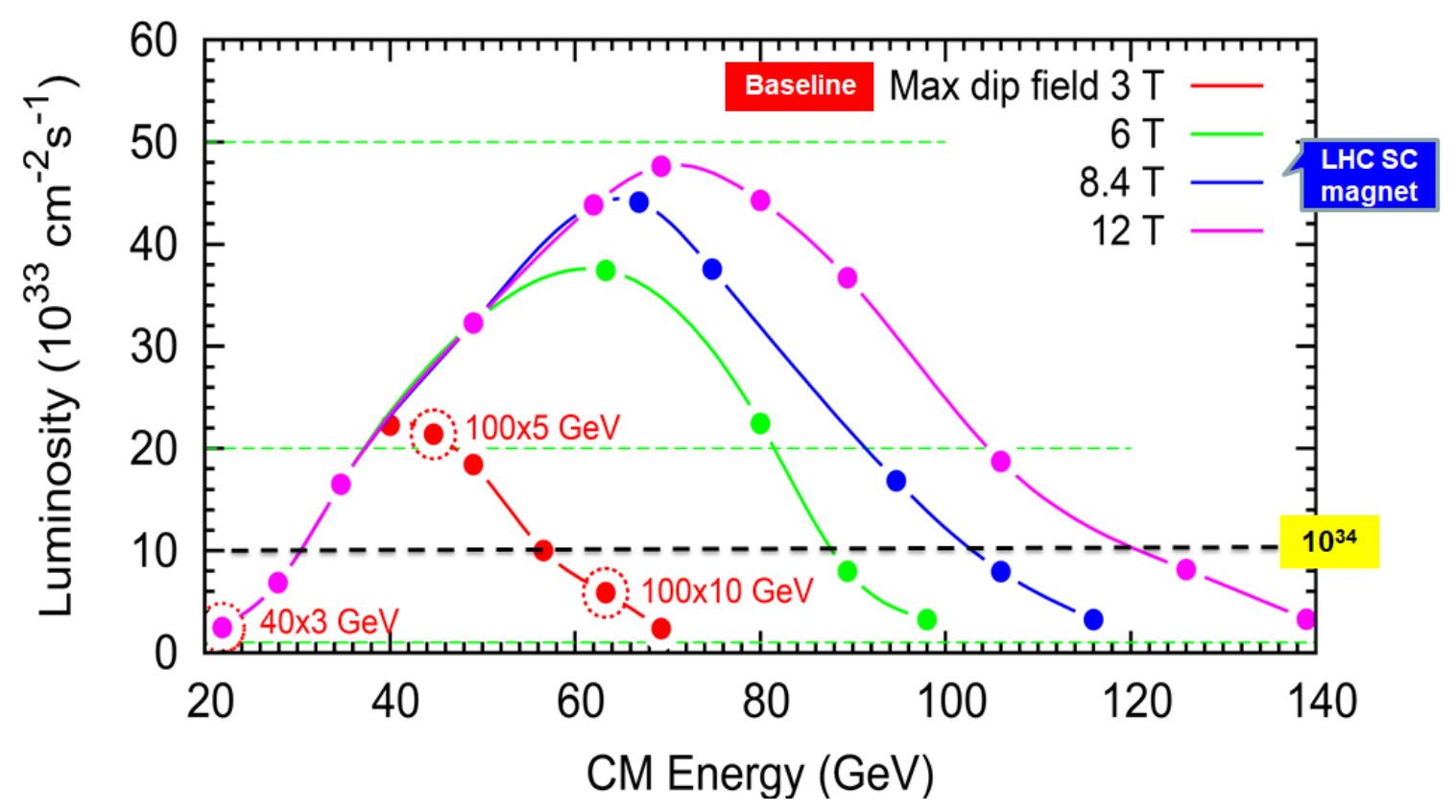

FIG. 6: Expected luminosity of JLEIC as a function of the center-of-mass energy. Different curves correspond to different beam bending (dipole) magnets [1].

- Small final focusing beta function at the collision point, $\beta^{*}$.

- Large beam-beam tune shift.

- Crab crossing of colliding beams.

The first three points specify requirements on the beams in terms of their phase space structure: the bunch length, beam emittance and time structure. The staged cooling of the ion beam is needed to achieve the small transverse beam emittance of the high energy ion beam. The last three items specify requirements on the interaction region that take advantage of the high repetition rate CW beam. Finally, the crab crossing of beams is required to compensate for luminosity loss due to a beam crossing. This dissertation is concerned with the crab crossing at JLEIC, which will be further described in Section 4.4 .

\subsubsection{POLARIZATION}

Another important requirement on JLEIC is the high polarization of beams over long times. This is required to study the spin distributions within QCD. In particular, the JLEIC polarization requirements are: 
- Over 70\% longitudinal polarization of both electron and light-ion beams at the interaction point.

- Transverse polarization and spin-flip of both beams are extremely desirable.

- Sufficiently long polarization times to sustain physics runs.

- Polarization of bunches can be switched at high frequency (polarization flipping).

- High precision (1-2\%) ion polarimetry.

The overall high-polarization approach at JLEIC relies on having figure-8 collider rings, which help preserve the starting polarization of the beams, particularly of the ion beam. The idea behind the figure- 8 rings [17] is the full cancellation of the spin precession at both arcs, therefore having a zero spin tune. A figure- 8 ring also provides better control of the electron beam polarization. The spin can further be controlled with spin rotators, which are arrangements of low-field solenoid mangets used to align the electron spins in the longitudinal direction near the interaction point and in the vertical direction at the arcs.

CEBAF is capable of producing fully polarized electron beams, which means that for the purpose of JLEIC, it is already a polarized electron beam injector. For the ion beam, however, polarized ion sources are required.

The polarization in the electron ring is preserved and enhanced through the SokolovTernov effect, in which a high-energy beam self-polarizes after traveling along a vertical strong magnetic field, through synchrotron radiation. In JLEIC, this corresponds to the beams traveling through the strong bending magnets in the arcs. In a figure- 8 ring, the spin rotates in one direction after passing through one of the arcs, but then it rotates back again when crossing the second arc. The resulting effect of both strong fields in the arcs is then effectively canceled, thus the zero spin tune. The figure-8 layout thus enables energyindependent spin tune and effectively eliminates depolarization at all spin resonances. This also means that during acceleration, the polarization of the beams is also preserved. For the ion beam, however, since there is no synchrotron radiation, there is no self-polarizing Sokolov-Ternov effect, but the spin can be controlled with Siberian snakes for protons and ${ }^{3} \mathrm{He}$, or special magnetic inserts for deuterons.

We have presented in this section the general JLEIC characteristics. We now present an overview of the required JLEIC facilities, which can be divided according to the beam particle species: electron and ion beam facilities. 


\subsection{ELECTRON COMPLEX}

The electron beam facilities for JLEIC consist of two general elements: the existing Continuous Electron Beam Accelerator Facility (CEBAF), which was recently upgraded to double its beam energy to $12 \mathrm{GeV}$, and the proposed JLEIC electron collider ring.

\subsubsection{CEBAF 12 GEV}

CEBAF is the main accelerator at Jefferson Lab [18. It is a $1.5 \mathrm{~km}$ long recirculating linac shaped as a race-track. It is formed by two $180^{\circ}$ arcs connected by two superconducting linacs. It delivers beam to four experimental halls, labeled A through D, where the electron beam is brought into collision with stationary targets to study the nuclei. CEBAF has recently started operations after an upgrade to double the electron beam energy from 6 to $12 \mathrm{GeV}$ [19]. Major aspects of the upgrade project included the upgrade of the injector, recommissioning of existing cryogenic accelerating modules and the addition of 10 new ones, upgrade of existing experimental halls and the addition of the new experimental Hall D, new arc sections were added, the spreaders and recombiners at the beginning and end of the arcs were rebuild and a new Helium liquifying plant was built. Figure 7 shows a layout of CEBAF and its upgraded components.

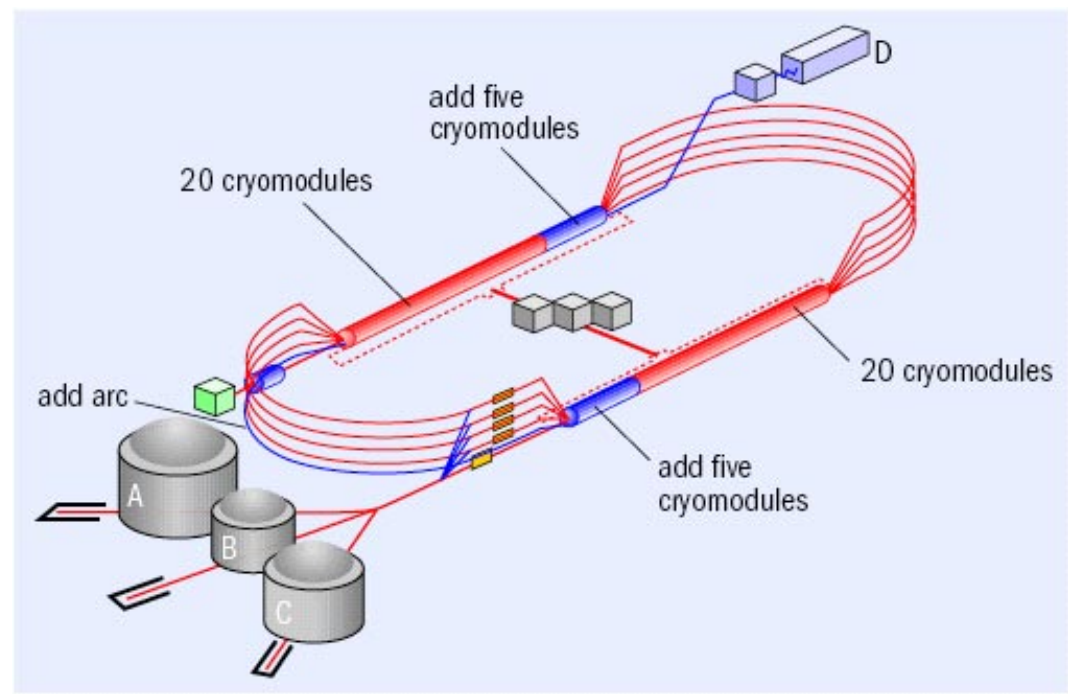

FIG. 7: Diagram of the Continuous Electron Beam Accelerator Facility (CEBAF) at Jefferson Lab and the elements added for the $12 \mathrm{GeV}$ upgrade.

CEBAF is a fixed target complex, which means the center-of-mass collision energy scales as $\sqrt{E_{\mathrm{b}}}$ and thus the probing energy reach is about a $1 \mathrm{GeV}$. On the other hand, it has the unique capability of simultaneously deliver beams with different polarization to each of the 
experimental Halls. This capability is based on the use of RF separators. This is illustrated in Fig. 8, where a $500 \mathrm{MHz}$ RF separator cavity is used to "divide" the $1.5 \mathrm{GHz}$ repetition rate beam and send each beam component into different paths leading to Halls A, B and C.

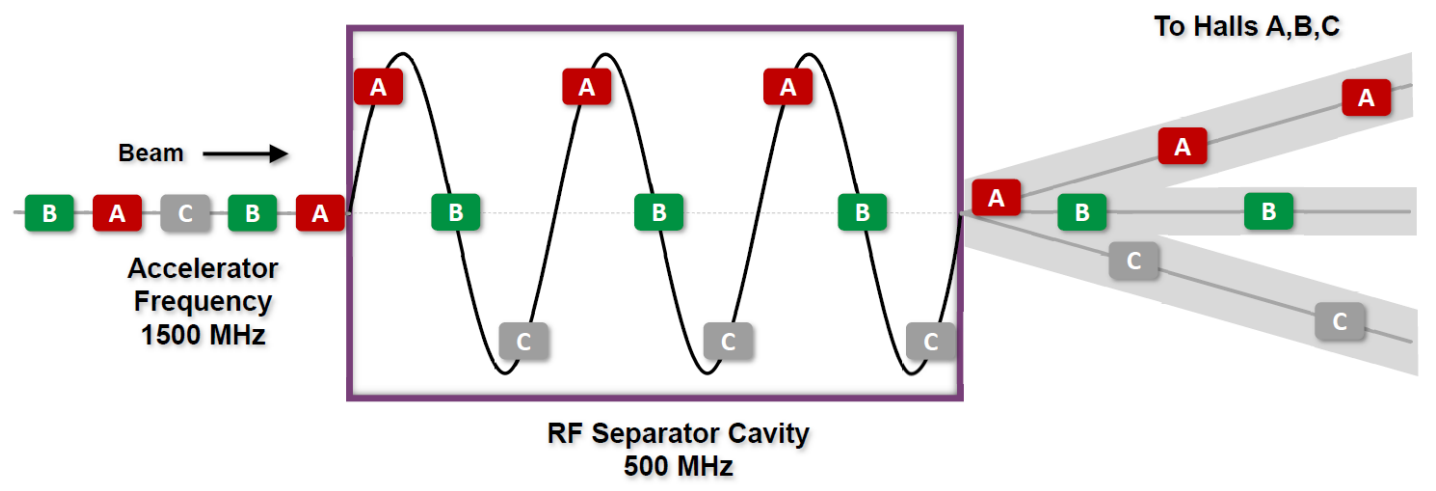

FIG. 8: A $500 \mathrm{MHz}$ RF separator cavity is used at CEBAF to separate and send the beams to Halls A, B and C.

A map of the beam path trough CEBAF is presented in Fig. 9, where the electron beam is created at the $130 \mathrm{keV}$ polarized electron gun. Electrons are stripped out of a GaAs cathode through the photo-electric effect. The beam is then accelerated up to $123 \mathrm{MeV}$ and sent through the injector chicane and into the North Linac. With each passage through a Linac, the beam gains up to $1090 \mathrm{MeV}$.

The arcs are formed by vertically stacked beam tubes with dipole magnets that bend the beam through the arcs. At the beginning of each arc the beam is sent through different paths depending on its energy. Similarly, at the end of each arcs, the beam is recombined before going into the linac. Low energy electron beams are sent through the upper beam-pipe in the arc. With each turn, the beam gains more energy and is sent through lower and lower arcs. Beams intended for Halls A, B and C circulate 5 times through CEBAF, for a total kinetic energy of $11 \mathrm{GeV}$, before being sent through the Hall switchyard, where the beam is separated to its respective Hall. The beam going to experimental Hall D is sent through the North linac an extra time, therefore achieving $12 \mathrm{GeV}$ energy beam only at Hall D.

For JLEIC, upgrades on the CEBAF RF systems and the electron beam injector are planned. Also, an extraction beam line will be added near Hall D, which will send the electron beam into the JLEIC electron collider ring. 


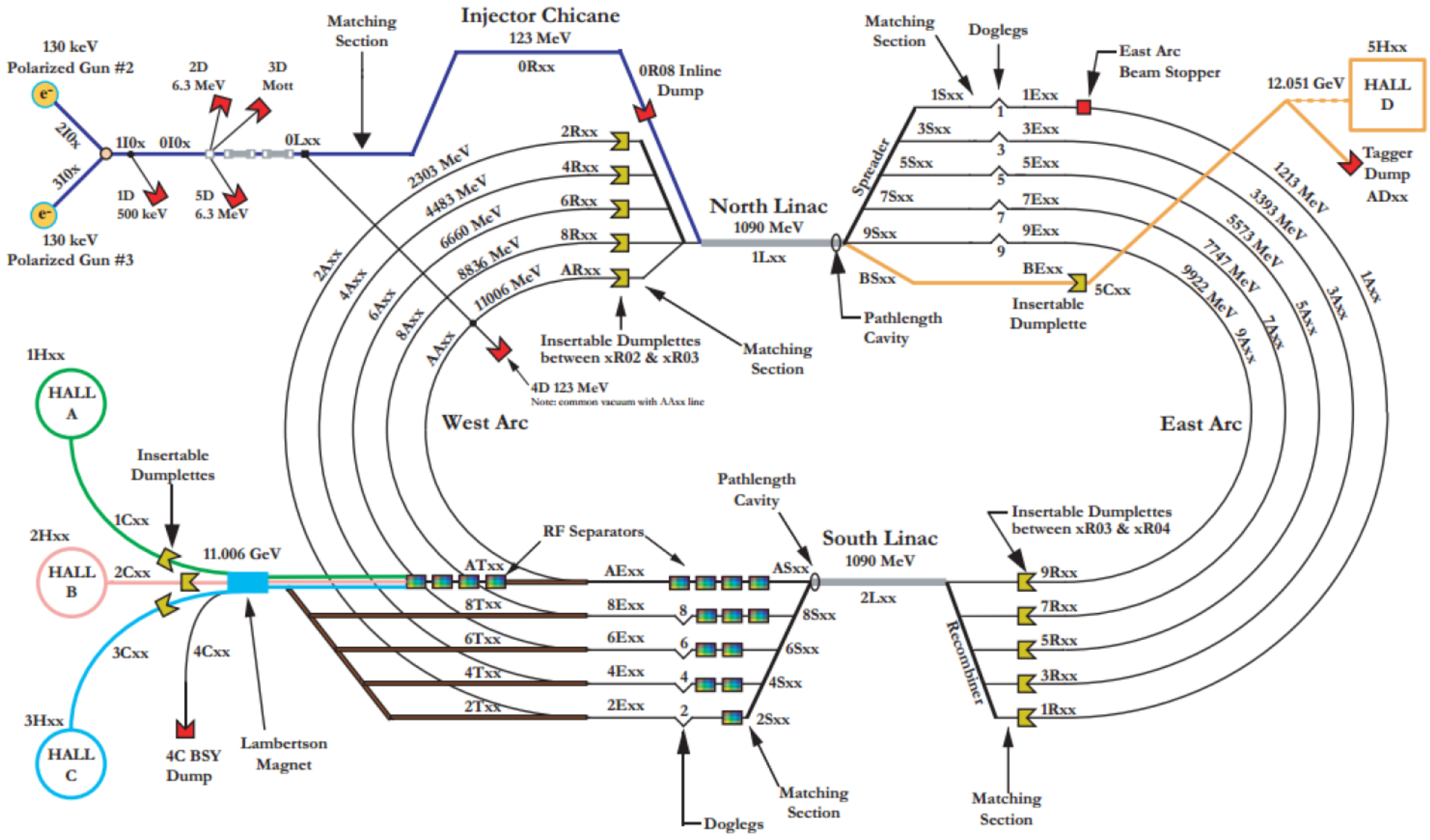

FIG. 9: Diagram of the beam path and its energy in CEBAF $12 \mathrm{GeV}$, from the electron gun to the experimental Halls A, B, $\mathrm{C}$ and D. 


\subsubsection{ELECTRON COLLIDER RING}

The JLEIC electron ring is designed with CEBAF $12 \mathrm{GeV}$ as a full energy electron beam injector. Although the change of frequency for JLEIC was motivated to reuse the PEP-II $\mathrm{RF}$ components, the most recent electron ring is based on new warm magnets and only the PEP-II RF cavities are now expected to see a second life at JLEIC. The electron ring has a circumference of $2498.64 \mathrm{~m}$, of which each arc is $956.24 \mathrm{~m}$ and each straight section is 293.1 $\mathrm{m}$ long. The arcs are formed by arrangements of focusing/defocusing quadrupole magnets, known as FODO cells, and warm bending magnets. The straight sections intersect at a $69^{\circ}$ angle. One of the straights has the space for the interaction region, while the other hosts the rf cavities and space for a future second detector. The FODO cell have a length of 11.4 m. Figure 10 shows the electron collider ring footprint with its main optical elements.

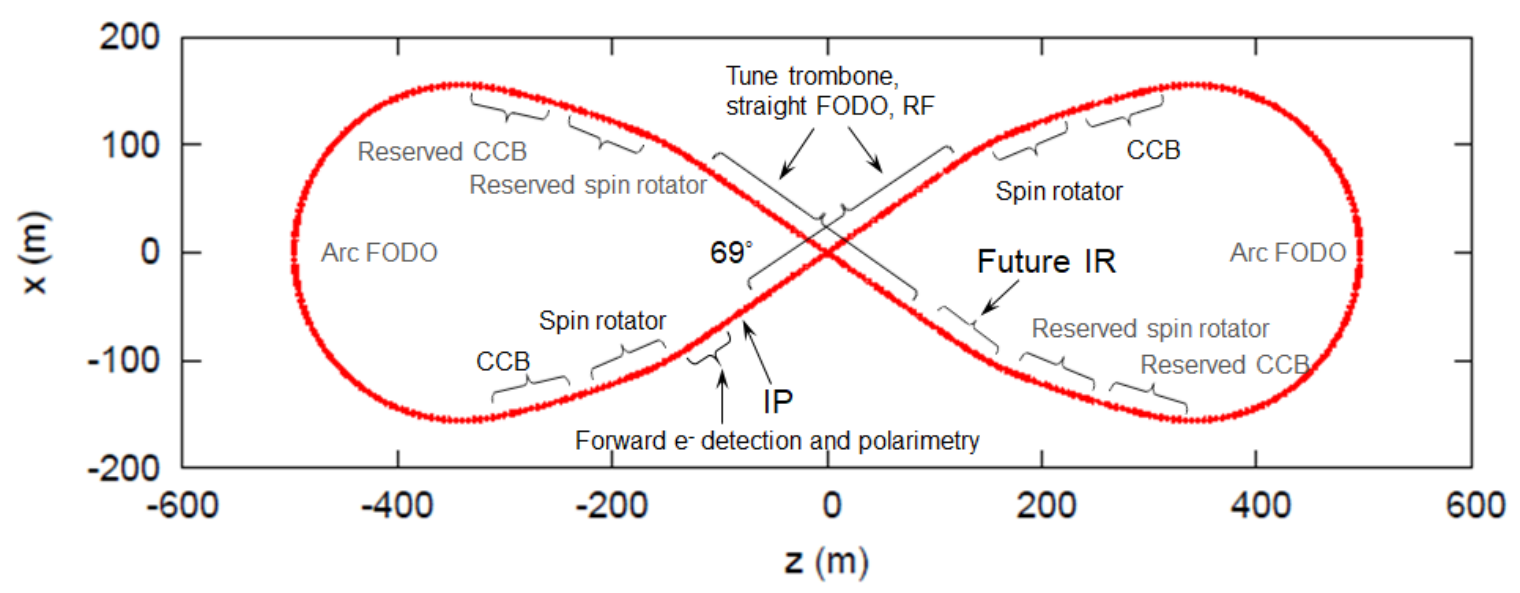

FIG. 10: Figure-8 electron collider ring for JLEIC and its main optical elements.

\subsection{ION COMPLEX}

Jefferson Lab is currently an electron beam science facility, and there are no existing ion beam facilities on-site. Because of this, the proposed infrastructure to support the JLEIC ion beam is being designed from the ground up. It can be divided into four major components: the ion source and linac, the ion ring booster, the ion collider ring and the ion beam cooler.

\subsubsection{ION SOURCE AND LINAC}

For the ion beam, two sources are being considered: a polarized ion source for proton and light-ions, and a non-polarized ion source for heavier ion species. These sources are available. 
After the ions are extracted from the source, they must be accelerated as quickly as possible to minimize space-charge effects, and especially to have a good beam emittance when the beam is injected into the booster ring. The first acceleration stage of the ion beam is done with a combination of normal conducting and superconducting linacs. Figure 11 shows the ion sources and linac for JLEIC [1].

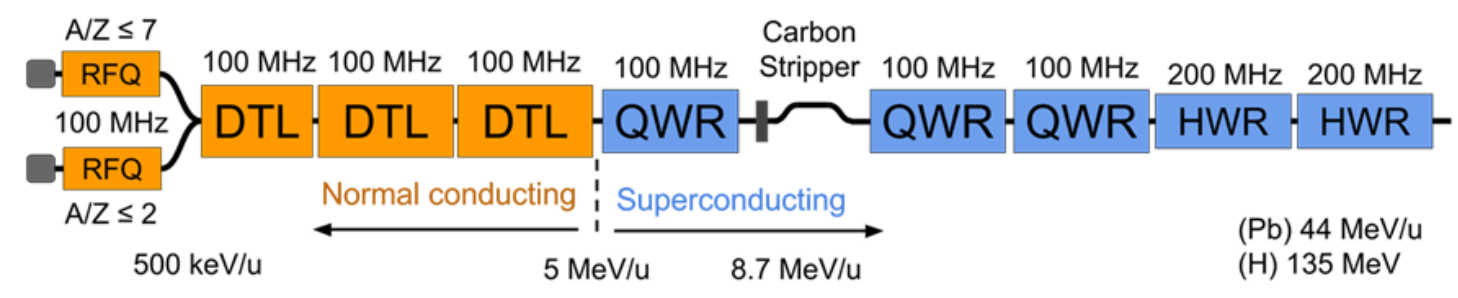

FIG. 11: Ion source and linac for the first-step acceleration of the ion beam.

The normal conducting part of the linac is based on $100 \mathrm{MHz}$ Drift Tube Linac, while the superconducting section uses a combination of $100 \mathrm{MHz}$ Quarter Wave Resonators (QWR) and $200 \mathrm{MHz}$ Half Wave Resonators (HWR).

\subsubsection{ION BOOSTER RING}

The linac injects the beam into the booster ring to further accelerate protons to $8 \mathrm{GeV}$, or ions to $3.2 \mathrm{GeV}$ per nucleon. The booster ring is also shaped to a figure- 8 on the same grounds previously discussed. It is based on super-ferric magnets. The booster ring was designed to
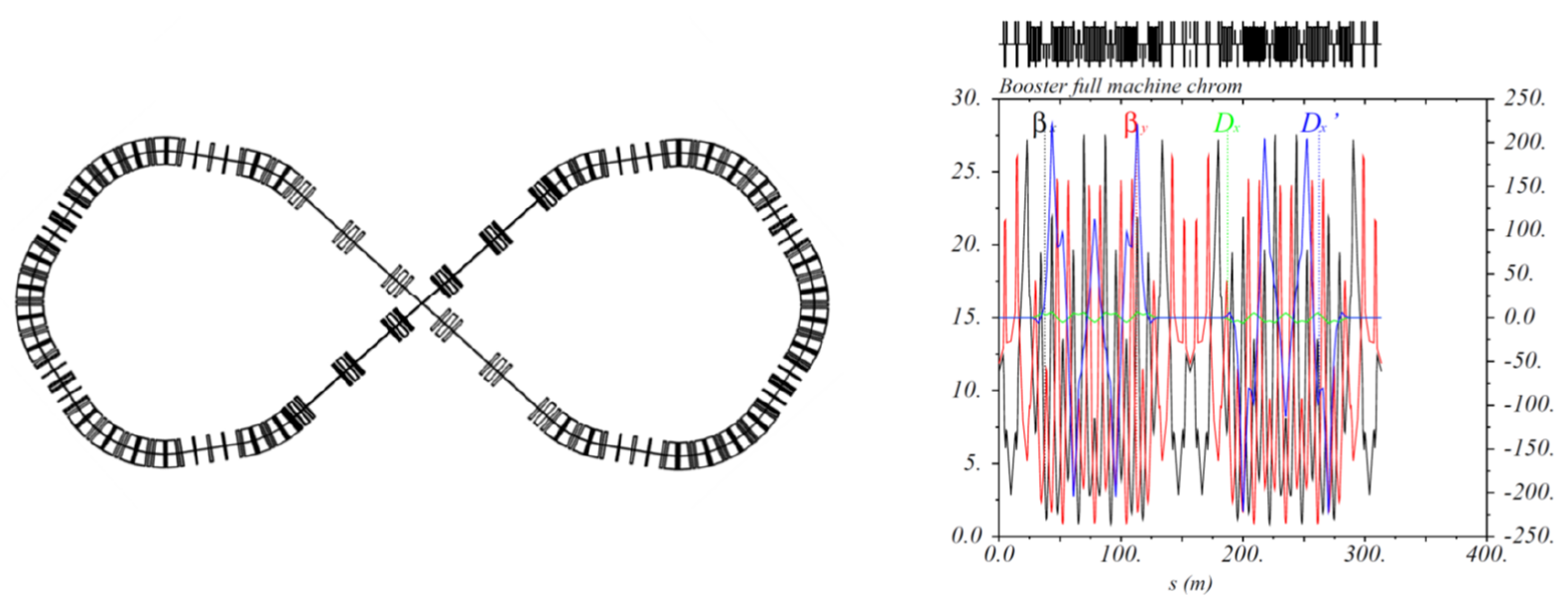

FIG. 12: Footprint of the ion booster ring and its corresponding optics.

avoid the crossing of transition energy $\gamma_{t}$ for any ion beam species, which minimizes the loss of particles during acceleration [20]. As part of the JLEIC high-luminosity plan, the use of 
ion beam cooling is needed to provide low-emittance ion beams for collision. A first stage of the cooling is done in the booster ring, where a conventional DC cooler is used to reduce the beam emittance to an appropriate level before being sent into the collider ring.

\subsubsection{ION COLLIDER RING}

The ion ring is where the ion beams will be accelerated to their higher energy, stored and brought into collision with the electron beam. It has a figure- 8 shape to match the electron ring tunnel and has a circumference of $2498.64 \mathrm{~m}$. It is formed by two arc sections and two straights, where the arcs are mainly FODO sections of $22.8 \mathrm{~m}$ each, which is twice as long as the FODO sections of the electron ring. The arc also has the bending magnets, which are based on super-ferric magnet technology. One of the straights hosts the interaction region, while the other one hosts the RF cavities and space for a future second detector. In the interaction region, the ion beam is diverted from the ion ring plane to meet the electron beam in its own plane. Figure 13 shows the ion ring footprint with its main elements, it also shows the overlap with the electron ring. In this case, the superconducting accelerating cavities

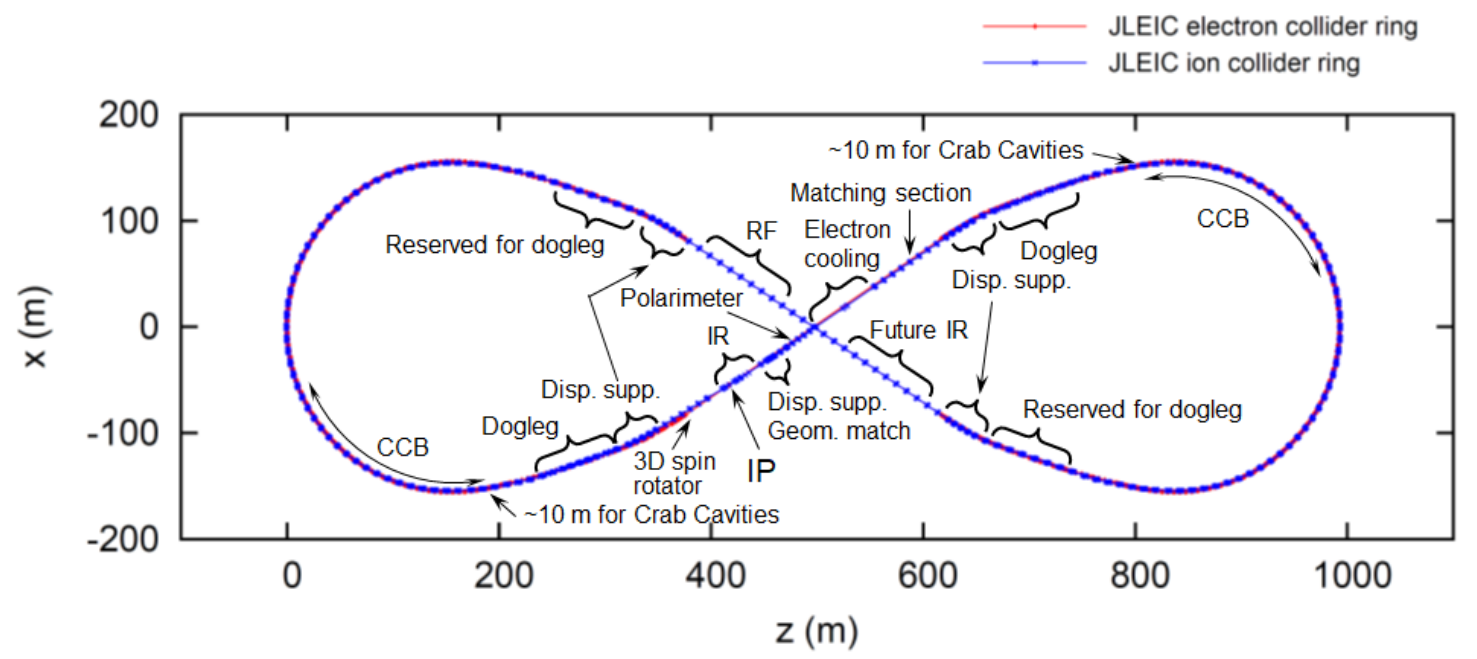

FIG. 13: Layout of the ion collider ring, which is matched to the electron ring. This ring has reserved $10 \mathrm{~m}$ of space for crab cavities at each side of the IR.

are being designed for this specific application, i.e., no reusing from a former machine. Since there is no self-polarization through Sokolov-Ternov effect because synchrotron radiation is minimum for the ion beam, the spin is controlled with spin rotators. Similarly to the electron ring, the ion ring has reserved space for a second detector to be added in a future upgrade. Together with the integration of crab cavities, which will be further discussed in Section 4.4 , 
the cooling of the high-energy beam in the ion collider ring is one of the most critical features to be implemented in JLEIC. It will be described in the following section.

\subsubsection{BEAM COOLING}

The JLEIC luminosity strategy relies on the use of staged electron cooling of the ion beam. A conventional DC cooler is used in the ion booster ring and a state-of-art bunched beam cooler is used in the ion collider ring. The bunched beam cooler is a small electron beam accelerator on its own. It is based on an energy recovery linac (ERL) concept and is used in the ion collider ring to lower the transverse beam emittance of the high-energy ion beam, which otherwise will grow over time due to intra-beam scattering (IBS).

The idea is to have an electron beam travel together with the high-energy ion beam along a section of the ion collider ring. By having both beams travel together, it is expected that the electromagnetic interaction with the electron beam will bring down the ion beam emittance. By reducing the beam emittance, smaller ion beams are brought into collision, thus increasing the luminosity. Figure 14 shows a diagram of the bunched beam cooler concept.

top ring: $\mathrm{CCR}$

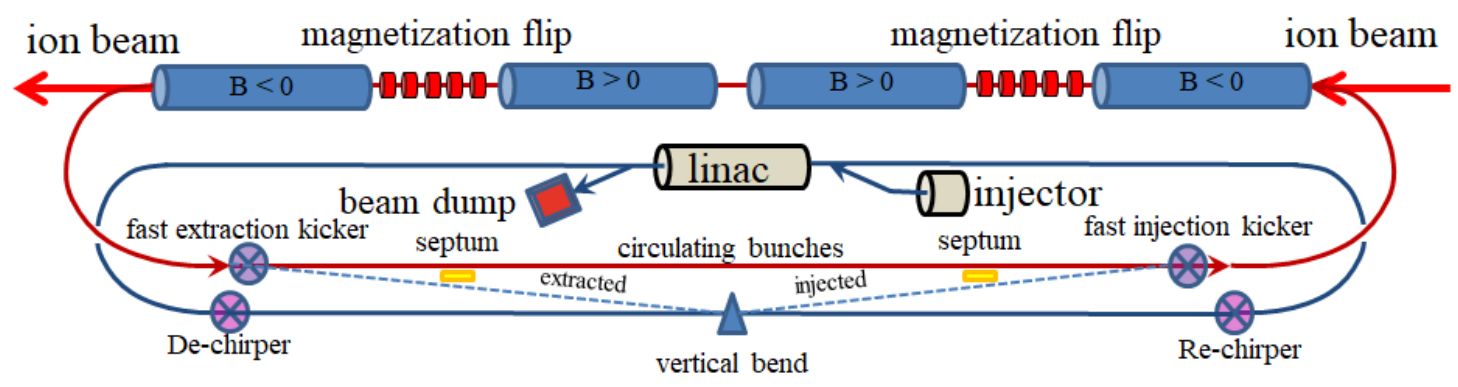

bottom ring: ERL

FIG. 14: Concept of bunched beam cooler for the high energy ion beam in JLEIC.

The bunched beam cooler is being designed as an Energy Recovery Linac (ERL), which allows the beam to deposit part of its power back into the linac cavities after being used and before being dumped. One of the technical challenges on the cooler technology is the generation of magnetized beam at the electron gun, which can be achieved by placing a strong solenoid magnet just outside of the electron gun. 


\subsection{INTERACTION REGION}

The interaction region of JLEIC is designed to achieve the high-luminosity collisions together with a full acceptance detector. The acceptance of a detector characterizes its ability to register collision products in the full solid angle spanning from the axis of the collisions. This, however, means placing detector components very close to the beam elements. By a careful design of the interaction region, an optimal compromise between detector and accelerator elements can be determined. Figure 15 shows a diagram of the interaction region, the detector and its solenoid magnet is aligned to the electron beam pipe and the ion beam meets the plane of the electron ring for the collision.

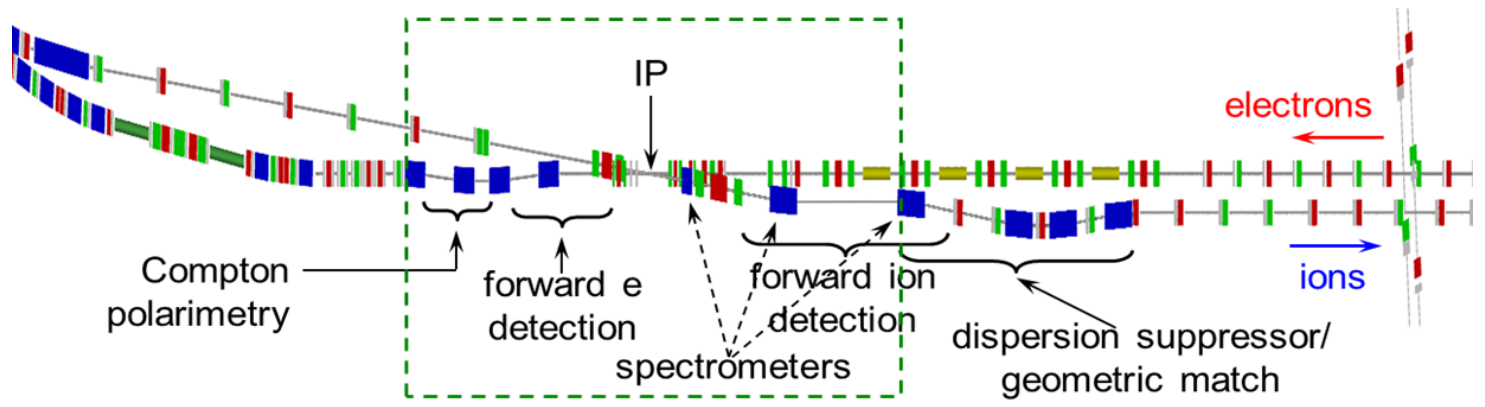

FIG. 15: Diagram of the interaction region of JLEIC. The ion beam meets the electron ring plane for the collision. The detector is aligned with the electron beam.

A relatively large crossing angle $\phi_{\text {cross }}=50 \mathrm{mrad}$ is adopted in the JLEIC design, which allows for a rapid separation of the two colliding beams near the interaction point. This also allows to place the final focusing quadrupoles (FFQ) closer to the interaction point. The FFQ are strong quadrupole field magnets used near the collision point that provide the final "squeeze" to the bunches before collision. The current interaction region design showcases a small $\beta^{*}$ of $10 \mathrm{~cm}$ at the interaction point, which is also one of the highluminosity strategy points, this is achieved by the FFQ. The need for strong fields at the FFQ magnets has another consequence, as the FFQ can introduce chromatic aberrations in the beam. The JLEIC interaction region is also designed with optical sections known as Chromaticity Compensation Blocks (CCB), which are pairs of sextupoles used to correct aberrations introduced in the beam by the FFQ. The interaction region also features the use of crab cavities, which are implemented in order to compensate the luminosity loss due to the $50 \mathrm{mrad}$ crossing angle. As it will be discussed in Section 4.4, the locations of horizontal CCB are compatible with the bunch crabbing parameters for JLEIC. 


\section{CHAPTER 3}

\section{SRF CAVITY FUNDAMENTALS}

This chapter introduces the fundamental physics behind radio-frequency cavities used in particle accelerators. It is intended to serve as a review of relevant concepts that will be discussed in following chapters. It is organized in the following way: we will discuss first in Section 3.1 the nature of the electromagnetic fields inside a resonant cavity and parameters of interest of a radio-frequency cavity. Section 3.2 is an overview of the superconducting radio-frequency (SRF) science and technology and the advantages of using superconducting over normal conducting materials. Finally, Section 3.3, presents specific applications of SRF crab cavities in colliders.

\subsection{ELECTROMAGNETIC WAVES IN CAVITIES}

Electrodynamics is a field theory that describes the complex interactions between an electric charge or current and its environment through electromagnetic fields. At its core, the dynamics is elegantly contained within the four Maxwell's equations [21]:

1. Gauss's Law. It states that given any surface enclosing an electric charge distribution $\rho$, the flux of electric field passing through the surface is proportional to the total electric charge contained:

$$
\nabla \cdot \mathbf{D}=\rho
$$

2. Null divergence of the magnetic field. This states that the magnetic field lines close around themselves forming loops, which in turn implies the non-existence of magnetic monopoles, i.e., there is no isolated "magnetic charge":

$$
\nabla \cdot \mathbf{B}=0
$$

3. Faraday's law. Also known as the induction law, it describes a spacial change of the electric field due to a time-varying magnetic field and vice versa:

$$
\nabla \times \mathbf{E}=-\frac{\partial \mathbf{B}}{\partial t} .
$$


4. Ampère's law. The law for circuits, it describes the spacial variations of a magnetic field due to an electric current and a displacement current term:

$$
\nabla \times \mathbf{H}=\mathbf{J}+\frac{\partial \mathbf{D}}{\partial t}
$$

In these equations, $\mathbf{E}$ is the electric field, $\mathbf{B}$ is the magnetic induction, $\rho$ is an electric charge distribution and $\mathbf{J}$ is a current distribution. The quantities $\mathbf{D}=\epsilon \mathbf{E}$ and $\mathbf{H}=\mu^{-1} \mathbf{B}$ describe the fields in a medium and are called the displacement field and magnetic field, respectively, with $\epsilon$ the electric permittivity and $\mu$ the magnetic permeability of the medium.

For our purpose of describing the electromagnetic fields inside a cavity, we are concerned about the fields in vacuum, where $\epsilon=\epsilon_{0} \approx 8.854 \times 10^{-12} \mathrm{C}^{2} / \mathrm{Nm}^{2}, \mu=\mu_{0}=4 \pi \times 10^{-7} \mathrm{~N} / \mathrm{A}^{2}$ are the permittivity and permeability of free space and

$$
c=\frac{1}{\sqrt{\epsilon_{0} \mu_{0}}} \approx 299,792,458 \mathrm{~m} / \mathrm{s}
$$

is the speed of light in free space. Furthermore, if there are no charge sources or currents present ( $\rho=0$ and $\mathbf{J}=0$ ), Maxwell's equations become

$$
\begin{aligned}
\nabla \cdot \mathbf{E} & =0 \\
\nabla \cdot \mathbf{B} & =0 \\
\nabla \times \mathbf{E} & =-\frac{\partial \mathbf{B}}{\partial t} \\
\nabla \times \mathbf{B} & =\frac{1}{c^{2}} \frac{\partial \mathbf{E}}{\partial t} .
\end{aligned}
$$

Other than the symmetric appearance of these equations, it can be noted that they form a system of coupled first-order differential equations for the fields $\mathbf{E}$ and $\mathbf{B}$. In order to find solutions, they can be decoupled in the following way: take the rotational on both sides of Eq. (8),

$$
\nabla \times(\nabla \times \mathbf{E})=-\frac{\partial}{\partial t}(\nabla \times \mathbf{B})
$$

use of vector identities ${ }^{1}$ on the left side term and substitute Eq. (9) on the right side to get

$$
\nabla(\nabla \cdot \mathbf{E})-\nabla^{2} \mathbf{E}=-\frac{1}{c^{2}} \frac{\partial^{2} \mathbf{E}}{\partial t^{2}}
$$

since $\nabla \cdot \mathbf{E}=0$ from Eq. (6), then we arrive at

$$
\nabla^{2} \mathbf{E}-\frac{1}{c^{2}} \frac{\partial^{2} \mathbf{E}}{\partial t^{2}}=0
$$

\footnotetext{
${ }^{1} \nabla \times \nabla \times \mathbf{A}=\nabla(\nabla \cdot \mathbf{A})-\nabla^{2} \mathbf{A}$
} 
A similar procedure starting with Eq. (9) yields an analogous expression for the magnetic induction,

$$
\nabla^{2} \mathbf{B}-\frac{1}{c^{2}} \frac{\partial^{2} \mathbf{B}}{\partial t^{2}}=0
$$

The differential equations (12) and (13) are now decoupled but of second-order. Furthermore, note that they both are wave equations, meaning the electromagnetic fields in vacuum propagate as waves.

\section{EM Waves at conducting boundaries}

In the boundary between vacuum and a conductor, the electromagnetic fields satisfy the following boundary conditions

$$
\begin{aligned}
\hat{n} \cdot \mathbf{D} & =\Sigma, \\
\hat{n} \times \mathbf{H} & =\mathbf{K}, \\
\hat{n} \cdot\left(\mathbf{B}-\mathbf{B}_{c}\right) & =0, \\
\hat{n} \times\left(\mathbf{E}-\mathbf{E}_{c}\right) & =0,
\end{aligned}
$$

where $\mathbf{E}_{c}$ and $\mathbf{B}_{c}$ are the electric field and magnetic induction inside the conductor; $\hat{n}$ is a unit vector that is normal to the surface of the conductor. A good conductor behaves similar to an idealized perfect conductor, where a charge $\Sigma$ and surface current $\mathbf{K}$ are distributed through a small, finite thickness at the surface, characterized by a skin depth $\delta$. Resistive losses in the surface can then be calculated, provided the fields of idealized (perfect conductor) are calculated [21].

\subsubsection{RESONANT CAVITIES}

At the radio frequency wavelengths that we are interested (frequencies in the $\mathrm{MHz}-\mathrm{GHz}$ range), the only practical way of generating and transmitting waves is through metallic structures of comparable dimensions.

It makes sense to propose plane wave solutions to the wave equations 12 and $(13)$, of the form:

$$
\begin{aligned}
& \mathbf{E}=\mathbf{E}(\rho, \phi) \exp [i(k z-\omega t)] \\
& \mathbf{B}=\mathbf{B}(\rho, \phi) \exp [i(k z-\omega t)]
\end{aligned}
$$

where $k$ is the wave number, $\omega$ is the angular frequency and they are both related through the dispersion relation $\omega=k c$. Substitution of Eqs. (18) and (19) into the wave equations 
(12) and (13) gives

$$
\begin{aligned}
& \nabla_{\perp}^{2} \mathbf{E}+\left(\frac{\omega^{2}}{c^{2}}-k^{2}\right) \mathbf{E}=0, \\
& \nabla_{\perp}^{2} \mathbf{B}+\left(\frac{\omega^{2}}{c^{2}}-k^{2}\right) \mathbf{B}=0 .
\end{aligned}
$$

The symbol $\nabla_{\perp}$ refers to the transverse component of the Laplace differential operator. Also, in selecting cylindrical coordinates $(\rho, \phi, z)$ for the plane wave solutions, we have already assumed the axial symmetry present on the most common radio-frequency cavity structures.

Because the boundary conditions need to be satisfied, the wave equations (inside the cavity) can only have a discrete set of solutions [22]. The wave solutions are known as the eigenmodes of the cavity, each with its characteristic eigenfrequency. These electromagnetic modes can be distinguished in two types: transverse electric (TE) and transverse magnetic (TM), according to which field is transversely oriented. Each electromagnetic mode is uniquely identified by using three numeric integers, one for each coordinate axis.

In general, radio-frequency cavities can have very complicated geometries and analytic solutions are not always available. There are, however, many computer codes that can be used to calculate the electromagnetic fields of cavities, for example CST Microwave Studio [5], which uses finite element methods to evaluate the electromagnetic properties. In the next section, we present a simple, yet educational cavity geometry that allows for an analytic solution.

\subsubsection{CYLINDRICAL CAVITY}

Consider the cylindrical cavity with radius $R$ and height $L$ shown in Fig. 16. The solutions for transverse magnetic modes $\mathrm{TM}_{m n p}$ are given by

$$
\begin{aligned}
& E_{z}=E_{0} \cos \left(\frac{p \pi z}{L}\right) J_{m}\left(\frac{x_{m n} \rho}{R}\right) \cos (m \phi), \\
& E_{\rho}=-E_{0} \frac{p \pi R}{L x_{m n}} \sin \left(\frac{p \pi z}{L}\right) J_{m}^{\prime}\left(\frac{x_{m n} \rho}{R}\right) \cos (m \phi), \\
& E_{\phi}=E_{0} \frac{m p \pi R^{2}}{\rho L x_{m n}^{2}} \sin \left(\frac{p \pi z}{L}\right) J_{m}\left(\frac{x_{m n} \rho}{R}\right) \sin (m \phi), \\
& H_{z}=0, \\
& H_{\rho}=i E_{0} \frac{m \omega_{m n p} R^{2}}{\eta c \rho x_{m n}^{2}} \cos \left(\frac{p \pi z}{L}\right) J_{m}\left(\frac{x_{m n} \rho}{R}\right) \sin (m \phi), \\
& H_{\phi}=i E_{0} \frac{\omega_{m n p} R}{\eta c x_{m n}} \cos \left(\frac{p \pi z}{L}\right) J_{m}^{\prime}\left(\frac{x_{m n} \rho}{R}\right) \cos (m \phi) .
\end{aligned}
$$




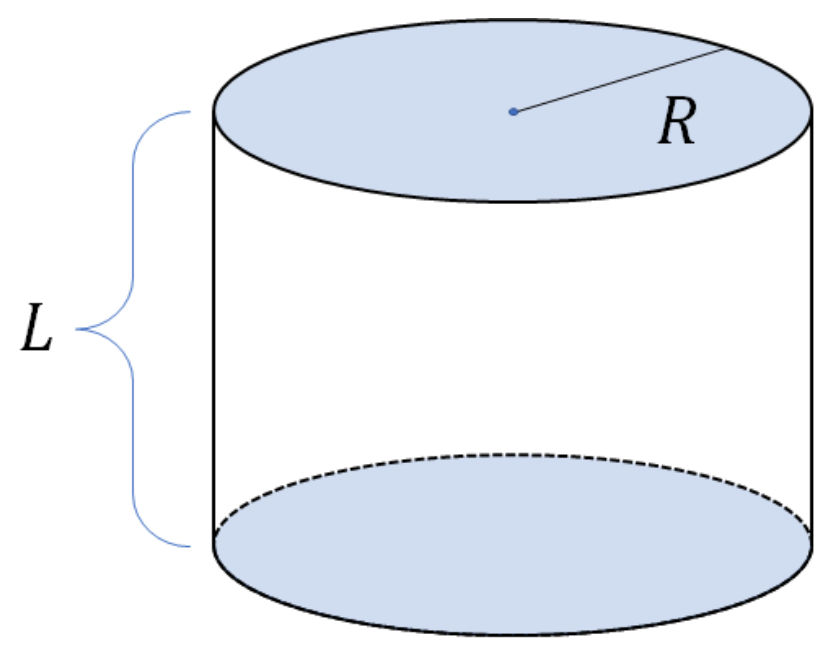

FIG. 16: Cylindrical cavity with radius $R$ and length $L$.

Similarly, the solutions for transverse electric modes $\mathrm{TE}_{m n p}$ are

$$
\begin{aligned}
H_{z} & =H_{0} \sin \left(\frac{p \pi z}{L}\right) J_{m}\left(\frac{x_{m n}^{\prime} \rho}{R}\right) \cos (m \phi), \\
H_{\rho} & =H_{0} \frac{p \pi R}{L x_{m n}^{\prime}} \cos \left(\frac{p \pi z}{L}\right) J_{m}^{\prime}\left(\frac{x_{m n}^{\prime} \rho}{R}\right) \cos (m \phi), \\
H_{\phi} & =-H_{0} \frac{m p \pi R^{2}}{\rho L x_{m n}^{\prime 2}} \cos \left(\frac{p \pi z}{L}\right) J_{m}\left(\frac{x_{m n}^{\prime} \rho}{R}\right) \sin (m \phi), \\
E_{z} & =0, \quad \\
E_{\rho} & =i H_{0} \frac{m \eta \omega_{m n p} R^{2}}{c \rho x_{m n}^{\prime 2}} \sin \left(\frac{p \pi z}{L}\right) J_{m}\left(\frac{x_{m n}^{\prime} \rho}{R}\right) \sin (m \phi), \\
E_{\phi} & =i H_{0} \frac{\eta \omega_{m n p} R}{c x_{m n}^{\prime}} \sin \left(\frac{p \pi z}{L}\right) J_{m}^{\prime}\left(\frac{x_{m n}^{\prime} \rho}{R}\right) \cos (m \phi), \\
\gamma_{m n} & =\frac{u_{m n}}{R}, \quad \omega_{m n p}=c \sqrt{\gamma_{m n}^{2}+\left(\frac{p \pi}{d}\right)^{2}},
\end{aligned}
$$

with $u_{m n}$ the $\mathrm{n}$-th root of the first-kind Bessel functions $J_{m}(x)$ and $J_{m}^{\prime}(x)$ is the derivative of $J_{m}(x)$ with respect to $x$.

\subsubsection{FIGURES OF MERIT}

Because of the rf fields in the cavity, currents are induced through a thin layer of the cavity surface in order to satisfy the boundary conditions. This rf currents dissipate energy in the cavity wall through Joule heating. The energy loss in the cavity is characterized by 
the surface resistance $R_{s}$, which is defined through the power dissipated per unit area [22]

$$
\frac{d P_{c}}{d s}=\frac{1}{2} R_{s}|\mathbf{H}|^{2}
$$

where $\mathbf{H}$ is the localized magnetic induction. Typical values of the surface resistance range from milli-ohms in Copper $(\mathrm{Cu})$ cavities to a few nano-ohms in a well prepared Niobium $(\mathrm{Nb})$ superconducting cavity. In general, the surface resistance may depend on the coordinates describing the cavity surface.

An important parameter of resonant cavities is the quality factor $Q_{0}$

$$
Q_{0}=\frac{\omega_{0} U}{P_{c}}
$$

where $U$ is the stored energy in the cavity and $P_{c}$ is the dissipated power in the cavity walls due to Joule heating. The quality factor can be interpreted as $2 \pi$ times the number of rf cycles needed to dissipate the stored energy in the cavity by a factor $e^{-1}$. The energy stored in the cavity can be calculated by

$$
U=\frac{1}{2} \mu_{0} \int_{V}|\mathbf{H}|^{2} d v=\frac{1}{2} \epsilon_{0} \int_{V}|\mathbf{E}|^{2} d v,
$$

where the integrated volume is defined by the cavity geometry. The dissipated power in the cavity surface can be estimated from Eq. (35)

$$
P_{c}=\frac{1}{2} \int_{S} R_{s}|\mathbf{H}|^{2} d s
$$

with $S$ the surface of the cavity. The quality factor can then be rewritten as

$$
Q_{0}=\frac{\omega_{0} \mu_{0} \int_{V}|\mathbf{H}|^{2} d v}{\int_{S} R_{s}|\mathbf{H}|^{2} d s} .
$$

In the special case where the surface resistance is constant everywhere on the cavity surface, it is convenient to write

$$
Q_{0}=\frac{G}{R_{s}}
$$

with

$$
G=\frac{\omega_{0} \mu_{0} \int_{V}|\mathbf{H}|^{2} d v}{\int_{S}|\mathbf{H}|^{2} d s}
$$

depends only on the cavity shape and is known as the geometry constant [22], $R_{s}$ on the other hand, depends only on material properties, thus the convenience of this simplified expression.

Other important cavity parameters are the operating frequency, which is the frequency of the mode in which the cavity is designed to work, and the beam aperture. The operating 
frequency determines the radius of the cavity cross section. In general, a larger beam aperture degrades the cavity performance, but a small aperture can lead to trapped modes in the cavity, which can in degrade the beam, as it will be explained in Chapter 6.

\subsubsection{SHUNT IMPEDANCE}

Another quantity used to characterize the losses on the cavity is the shunt impedance $R_{a}$, defined as [22]

$$
R_{a}=\frac{V_{c}^{2}}{P_{c}}
$$

in units of Ohm per cell. This is known as the accelerator definition, and is not to be confused with the circuit theory definition, which has a factor $1 / 2$ in front,

$$
R_{a}=\frac{V_{c}^{2}}{2 P_{c}}
$$

or the linac definition

$$
r_{a}=\frac{V_{c}^{2}}{P_{c}^{\prime}},
$$

where $P_{c}^{\prime}$ is the power dissipated per unit length.

The quantity $R_{a} / Q_{0}$ (read "R over Q") is independent of the cavity size. It determines how excitable a cavity mode is by a particular charge distribution passing through the cavity.

\subsection{SUPERCONDUCTING RF}

Two observed properties of superconductors are the zero resistance to DC currents and the expulsion of magnetic induction below a critical temperature $T_{\mathrm{c}}$. For AC currents, there exists a small, but finite resistance. The physics of superconducting materials is described by the theory of superconductivity proposed in 1957 by Bardeen, Cooper and Schrieffer [23]. In this theory, superconductivity is explained by the condensation of free electrons in the metal into Cooper pairs below $T_{\mathrm{c}}$. In the BCS theory, the surface resistance $R_{s}$ is proportional to the density of unpaired (normal) electrons in the metal, which increases exponentially with temperature. In the region $T<T_{\mathrm{c}} / 2$ and at low frequencies, a good approximation of the surface resistance is 22$]$

$$
R_{s}=A\left(\frac{1}{T}\right) f^{2} \exp \left[-\frac{\Delta(T)}{k T}\right]+R_{0}
$$

where $A\left(\frac{1}{T}\right)$ is a function of the material, $\Delta(T)$ is the superconducting energy gap: the energy needed to break a Cooper pair; $k$ is the Boltzmann constant and $R_{0}$ the residual resistance, which accounts for any other sources of dissipation. 
The two relevant properties of superconducting cavities that made them a good choice for particle acceleration are the high accelerating field $E_{a c c}$ and a high quality factor $Q_{0}$. A well-prepared, typical $1.5 \mathrm{GHz}$ accelerating cavity has $Q_{0}$ in the order of $10^{10}$ at $2 \mathrm{~K}[22$. The quality factor of an SRF cavity is inversely proportional to the surface resistance $R_{s}$ of the metal, which follows from Eq. (40). The surface resistance quantifies the amount of rf power being dissipated in the surface of the cavity. A low surface resistance, or high $Q$ is ideal, since less power dissipated in the cavity means less cryogenic load to keep the cavity at operating temperature. Most, if not all of the surface treatments in the SRF field have the purpose of reducing the surface resistance of a cavity, therefore reducing the cryogenic needs and cost of operation of an accelerator.

For a material to be of practical use for particle accelerators, it must have a high critical temperature $T_{\mathrm{c}}$, as well as a high super-heating magnetic field $H_{\mathrm{sh}}$, which defines the threshold for vortex penetration through the superconducting material. These vortices generate local regions of normal conducting material, thus becoming significant sources of dissipation in the surface. Table 2 shows superconducting parameters of the most common materials used for SRF applications. Of these, Nb has the highest lower critical field and is technologically the better-understood. It is the standard material of choice for SRF cavities.

TABLE 2: Superconducting materials of interest for rf applications.

\begin{tabular}{|c|c|c|c|c|c|c|}
\hline & $T_{c}[\mathrm{~K}]$ & $H_{c}[\mathrm{mT}]$ & $H_{c 1}[\mathrm{~T}]$ & $H_{c 2}[\mathrm{~T}]$ & $\lambda[\mathrm{nm}]$ & $\Delta[\mathrm{meV}]$ \\
\hline $\mathrm{Nb}$ & 9.2 & 0.2 & 170 & 0.4 & 40 & 1.5 \\
\hline $\mathrm{NbN}$ & 16.2 & 0.23 & 20 & 15 & 200 & 2.6 \\
\hline $\mathrm{Nb}_{3} \mathrm{Sn}$ & 18 & 0.5 & 40 & 30 & 85 & 3.1 \\
\hline
\end{tabular}

\subsection{CRAB CAVITIES}

In this section, we present a very specific application of srf technology in accelerators: the superconducting crab cavities used to increase the luminosity in particle colliders. Although the research on superconducting crab cavities for colliders is very active, only a few cavities have been integrated to accelerator rings: the crab cavity at KEKB for electron beams starting in 2007 and the recent crab cavity tests for the high luminosity LHC upgrade in 2018. We will discuss these together with the crab cavity for JLEIC.

Crabbing or deflecting cavities are superconducting rf structures designed to work either on a TE or TM mode. In essence, crab cavities are used to change the transverse momentum 
of the particles in a bunch. The transverse momentum gained by a charge $q$ particle is given by

$$
\Delta p_{\perp}=\int_{-\infty}^{\infty} \mathbf{F}_{\perp} d t
$$

where $\mathbf{F}_{\perp}=q\left[\mathbf{E}_{\perp}+(\mathbf{v} \times \mathbf{B})_{\perp}\right]$ is the transverse component of the Lorentz force. If the longitudinal velocity is larger than the transverse component, then

$$
\Delta p_{\perp}=\frac{q}{v} \int_{-\infty}^{\infty}\left[\mathbf{E}_{\perp}+(\mathbf{v} \times \mathbf{B})_{\perp}\right] d z
$$

where the integral now runs along the axis of the cavity. In a TE crab cavity like the rf-dipole, the transverse voltage is given by

$$
V_{\mathrm{T}}=\int_{-\infty}^{\infty}\left[E_{x} \cos \left(\frac{\omega z}{c}\right)+c B_{y} \sin \left(\frac{\omega z}{c}\right)\right] d z
$$

with $E_{x}$ is the horizontal electric field and $B_{y}$ is the vertical magnetic induction along the axis.

The length $L$ of the cavity is chosen to be multiples of half wavelengths $L=n \lambda / 2$, with $n$ the number of cavity cells and $\lambda$ the wavelength. This constraint on the cavity length allows to maximize the effect the cavity has on the particles, by ensuring they always see the electromagnetic field in the same direction.

\section{Panofksy-Wenzel theorem}

The Panofsky-Wenzel theorem neatly relates the transverse momentum kick acting on the particles to the transverse gradient of the accelerating field of the cavity [24],

$$
\Delta p_{\perp}=\frac{1}{v} \int_{-\infty}^{\infty} \mathbf{F}_{\perp} d z=-i \frac{q}{v} \int_{-\infty}^{\infty} \nabla_{\perp} \mathbf{E}_{z} d z
$$

\subsubsection{CRABBING OR DEFLECTING CAVITIES}

Crabbing or deflecting cavities are optimized according to its particular machine requirements and application, but in a general sense, the main difference between them is the phase of the rf field, which in turn determines the field that the bunch "sees" when it gets to the center of the cavity. Figures 17 and 18 illustrate the difference between a deflecting and crabbing cavity. In the deflector case, the phase of the cavity is such that the field is maximum at the bunch center, effectively imparting the same transverse kick along the bunch, therefore deflecting it. The rf separator of CEBAF shown in Fig. 8 is an example of a deflecting cavity. 


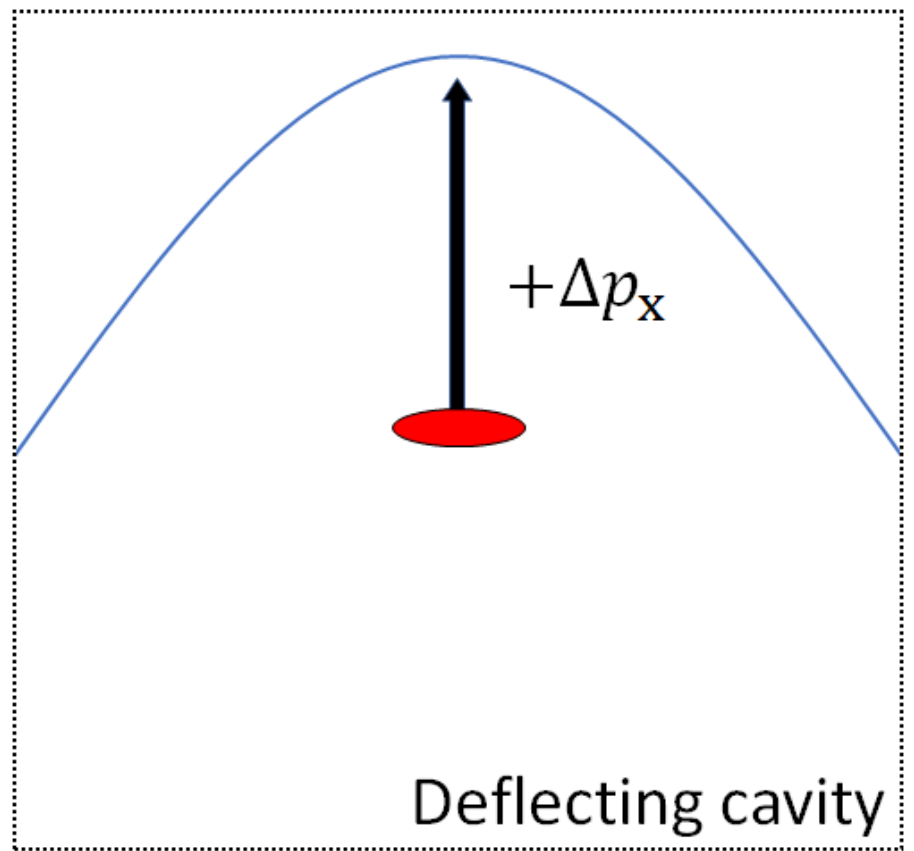

FIG. 17: In a deflector, the rf phase is such that the maximum transverse kick happens at the bunch center.

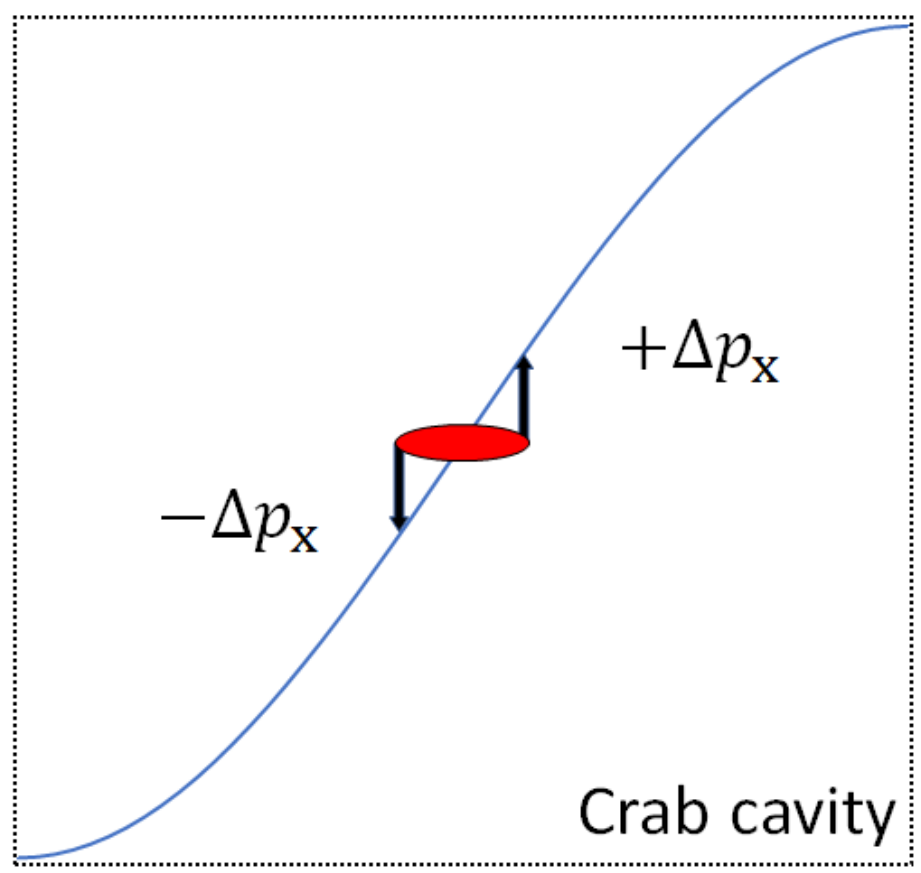

FIG. 18: In a bunch crabber, the phase is set up such that the field crosses zero at the bunch center. 
In the crab cavity case, the phase of the cavity is set up so that the field is zero at the bunch center. Because the field crosses zero at the center of the bunch, the head and tail get a transverse kick with the same magnitude but in opposite directions. The net effect is to produce a tilt of the bunch with no deflection.

\subsubsection{CRAB CAVITY EXPERIENCE AT KEKB}

KEKB was a B-factory in Japan that operated from December 1998 to June 2010 [2]. It was an energy-asymmetric $e^{+} e^{-}$collider composed of a low-energy ring (LER) for $3.5 \mathrm{GeV}$ positrons and a high-energy ring (HER) for $8 \mathrm{GeV}$ electrons. Beams were collided with an $11 \mathrm{mrad}$ crossing angle.

Crab cavities were first considered as a back-up strategy, and the KEKB high-luminosity strategy relied on a careful selection of the betatron tunes in order to have a high vertical beam-beam tune shift $\xi_{y}[2]$. Later, however, crab cavities were installed in February 2007 with the motivation of increasing the beam-beam parameter from its design value 0.052 to 0.15 , although a lower 0.129 was actually achieved. Table 3 summarizes the crabbing parameters used in KEKB.

TABLE 3: Parameters used for crabbing of electron and positron bunches in KEKB [2].

\begin{tabular}{|c|c|c|c|}
\hline Parameter & LER & HER & Unit \\
\hline Crossing angle & \multicolumn{2}{|c|}{22} & $\mathrm{mrad}$ \\
\hline$\beta_{x}^{*}$ & 1.2 & 1.2 & $\mathrm{~m}$ \\
\hline$\beta_{x}^{\text {crab }}$ & 51 & 122 & $\mathrm{~m}$ \\
\hline$\nu_{x}$ & 45.506 & 44.511 & $\mathrm{~m}$ \\
\hline$\psi_{x}^{\text {crab }} / 2 \pi$ & 0.25 & 0.25 & $\mathrm{~m}$ \\
\hline Crab voltage & 0.97 & 1.45 & MV \\
\hline Frequency & \multicolumn{2}{|c|}{508.89} & $\mathrm{MHz}$ \\
\hline
\end{tabular}

KEKB became the first collider to use crab cavities for increasing the luminosity of collisions and achieved the highest luminosity so far: $2.108 \times 10^{34} \mathrm{~cm}^{-2} \mathrm{~s}^{-1}$ during June of 2009 [2]. A local crabbing scheme was initially considered, but a global crabbing scheme was ultimately adopted on the grounds of saving cavity cost and taking advantage of available cryogenic infrastructure for the superconducting accelerating cavities [2]. The KEKB crab cavity was a single $508.89 \mathrm{MHz}$ superconducting squashed cavity, operating in the $\mathrm{TM}_{110}$ mode. A diagram of the cavity geometry is shown in Fig. 19. 


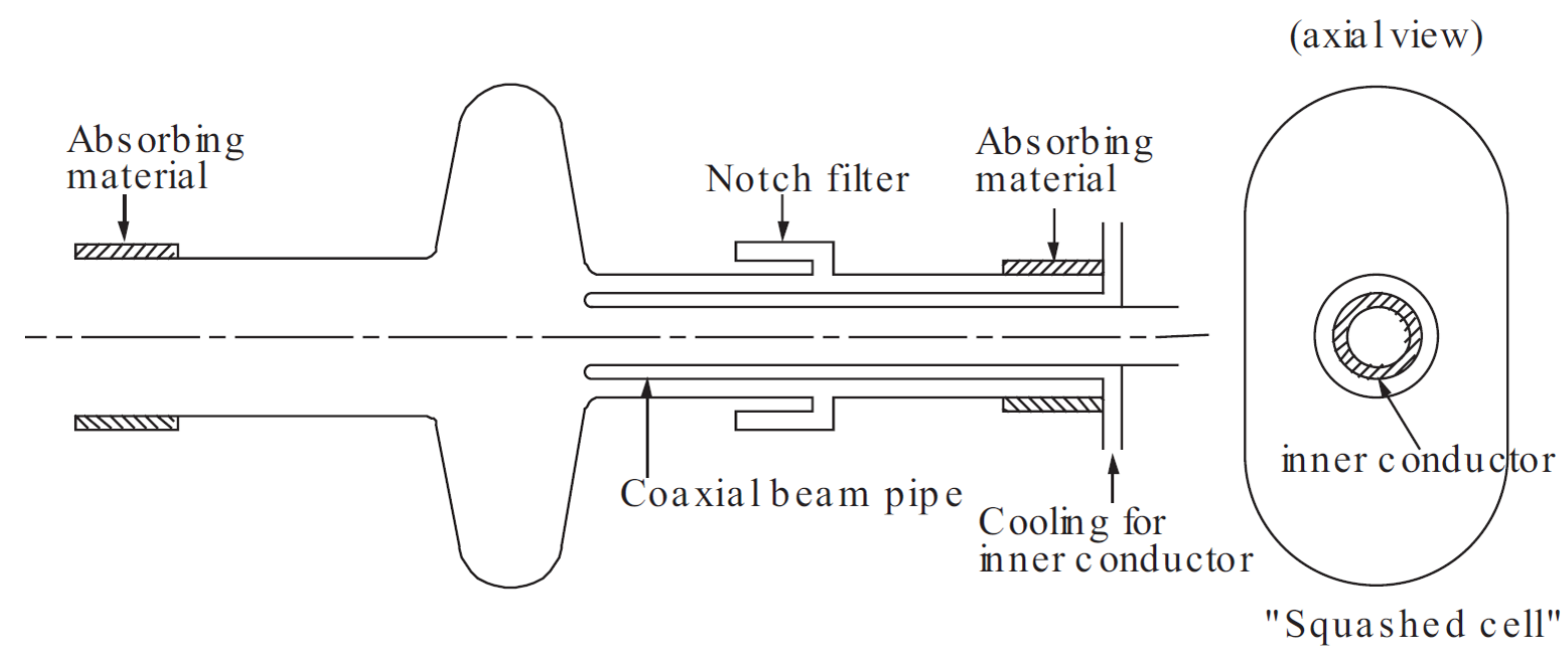

FIG. 19: Diagram of the squashed elliptical crab cavity used in KEKB.

\subsubsection{CRAB CAVITY TEST IN SPS}

The High-Luminosity upgrade of the Large Hadron Collider (HL-LHC) [14] includes the use of crab cavities for ATLAS and CMS. Two different concepts of crab cavities are being developed for this project, the double quarter wave (DQW) crab cavity [25] and the rf-dipole (RFD) cavity [26]. Both cavities are shown in Fig. 20.
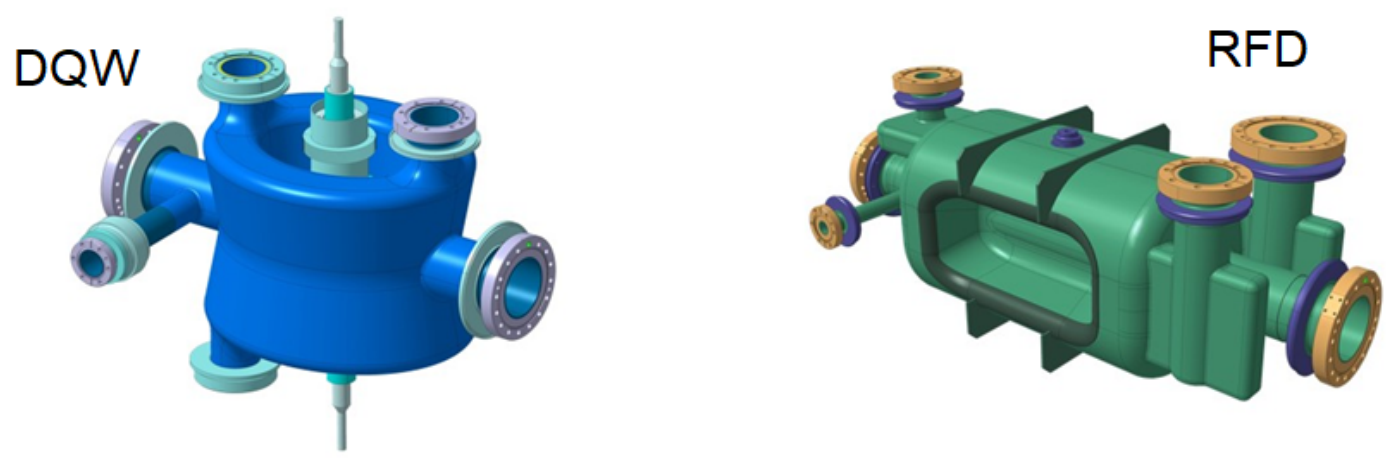

FIG. 20: Crab cavity concepts for the HL-LHC upgrade, DQW and RFD cavities for vertical and horizontal bunch crabbing.

As part of the crab cavity R\&D efforts for HL-LHC, numerous tests using $400 \mathrm{MHz}$ crab cavities in the Super Proton Synchrotron (SPS) accelerator ring are planned. The first beam tests will be done using two DQW crab cavities. On May $23^{\text {rd }} 2018$, the very first dedicated beam test using a single $26 \mathrm{GeV}$ proton bunch was performed, which served to demonstrate 
the crabbing of ions for the first time. With only one of the two crab cavities operating at $10 \%$ of its nominal voltage, the crabbing of a proton bunch was confirmed using a special monitor to track the tilt along the bunch length, see Fig. 4. The nominal voltage for the SPS tests using both crab cavities will be commissioned over several months. One of the main goals of the SPS test is to demonstrate the "transparency" of the crab cavities when detuned, as crab cavity testing should not interfere with normal LHC operation.

Similar tests using rf-dipole crab cavities in the SPS ring are also planned in coming years.

\subsubsection{CRAB CAVITY FOR JLEIC}

Crab cavities will have a central role on the delivery of high-luminosity collisions at JLEIC, and substantial research has been done in the Center for Accelerator Science at ODU. Different single-cell rf-dipole crab cavities have been designed and tested at ODU and Jefferson Lab, like a $400 \mathrm{MHz}$ HL-LHC prototype [27] and the $750 \mathrm{MHz}$ prototype for MEIC [28, 29], shown in Fig. 21. These cavities were tested vertically at Jefferson Lab and found to have good srf properties [30, 31].

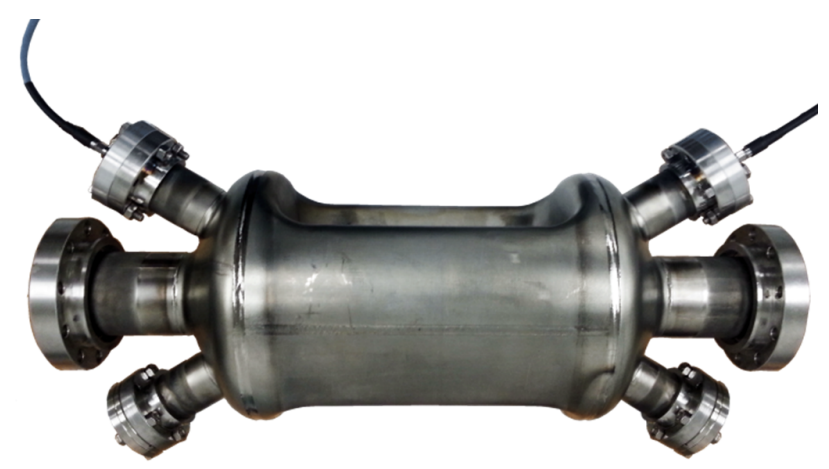

FIG. 21: Prototype of a $750 \mathrm{MHz}$ rf-dipole crab cavity for MEIC.

A crab cavity for the new frequency requirement of JLEIC has not yet been built, but many cavity designs are being studied. Two main things drive the general design geometry of the JLEIC crab cavity: one is the high voltage required for the crabbing of high-energy ion bunches, and the second is the limited space in the ion ring to host them. If we take for example a single-cell $952.6 \mathrm{MHz}$ rf-dipole cavity, the number of single-cell cavities required to produce the crabbing voltage makes this design prohibitive. A clever turn around to have a relatively low number of cavities and still produce a high crabbing voltage is the use of multi-cell structures [32]. Different 2-cell and 3-cell rf-dipole crab cavity concepts for JLEIC 
are currently being studied [33]. For a 3-cell cavity, the effective cavity length is $3 \lambda / 2$ with $\lambda$ the wavelength. Figure 22 shows a longitudinal cut of a 3-cell cavity with the electric field lines corresponding to the fundamental $\mathrm{TE}_{110}$ mode.

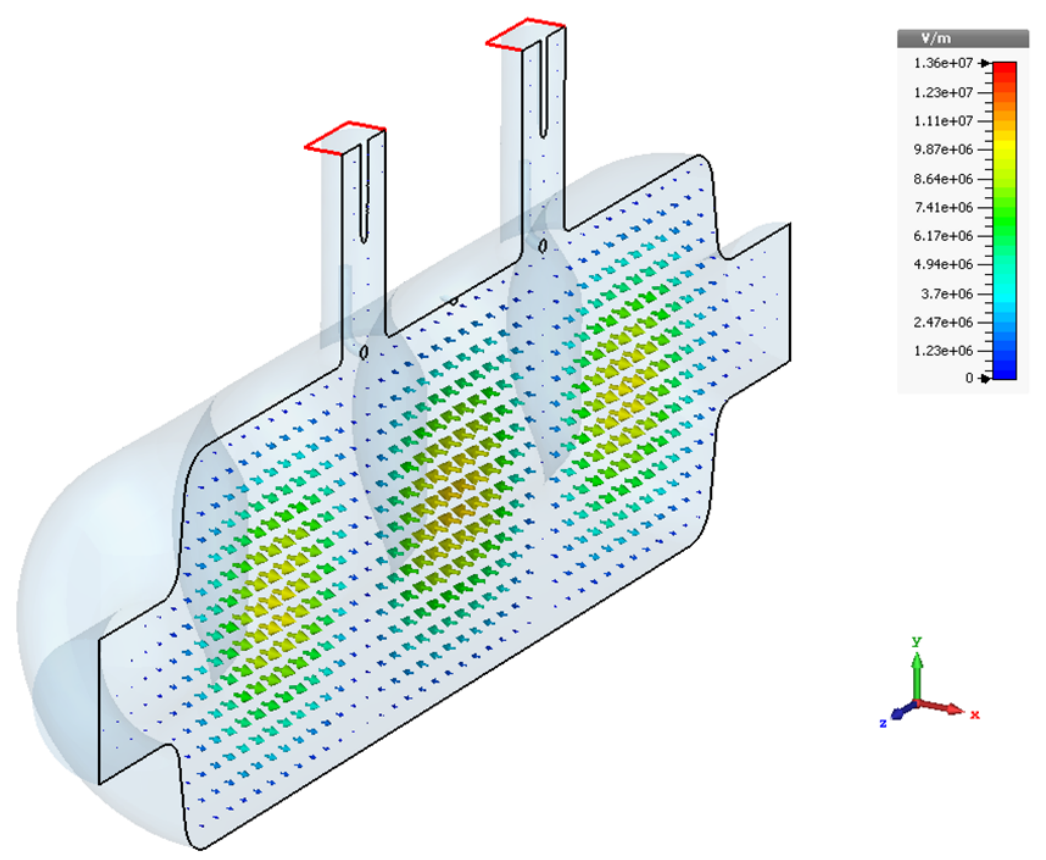

FIG. 22: Multi-cell rf-dipole crab cavity concept for JLEIC modeled in CST Microwave Studio [5], the arrows show the electric field lines. 


\section{CHAPTER 4}

\section{BEAM DYNAMICS AND CRAB CROSSING}

The goal of this chapter is to present a bunch crabbing scheme that is compatible with the JLEIC collider rings. With this objective in mind, Section 4.1 presnts an overview of the relevant concepts of beam dynamics like the transverse motion of particles in an accelerator and the beam emittance. Section 4.2 discusses the concept of luminosity of a particle collider, which is needed to present the luminosity reduction due to a beam crossing angle at the interaction point. Section 4.3 discusses the bunch crabbing scheme used in colliders to compensate geometric luminosity loss due to the beam crossing angle. Finally, in Section 4.4 we describe the characteristics of the local crabbing scheme under considerations for JLEIC.

\subsection{BEAM DYNAMICS}

All the dynamics of a beam of particles contained in a particle accelerator relies on the interaction of charged particles with both static and time-varying electromagnetic fields. Particles in an electromagnetic field are subject to the Lorentz force

$$
\mathbf{F}=q[\mathbf{E}+\mathbf{v} \times \mathbf{B}]
$$

with $q$ is the charge of the particle, $\mathbf{E}$ is the electric field, $\mathbf{v}$ is the particle velocity and $\mathbf{B}$ is the magnetic induction. We will see that based on this interaction, a beam of particles in a particle accelerator can be manipulated in a similar way as a light beam can be manipulated using for example lenses or collimators.

In modern accelerators, particles gain their energy from time-varying electric fields, while static magnetic fields are used to bend, focus and defocus the beam of particles. In this sense, dipole magnets are used to bend the beam into any desired direction, e.g. to follow an arc section of a ring. Quadrupole magnets are used to both focus or defocus the beam, depending on the beam design. Modern synchrotron rings are built with basic "blocks" known as FODO cells, which are arragnements of focusing and defocusing quadropoles that have a net fosusing effect of the beam, this principle is called strong focusing [34]. Sextupole, octupole or decapole magnets can also be used to correct higher order optical aberrations. 
When designing a particle accelerator, one first designs an ideal beam trajectory. Of course in reality, particles will generally not follow this precise trajectory, but rather it will be shown that the motion of particles can be described relative the the ideal beam trajectory. The motion of a beam can be separated between the longitudinal and the transverse dynamics of the beam. Figure 23 shows an arbitrary design trajectory for a particle at the origin. For convenience, we define a system of coordinates such that the longitudinal coordinate $s$ follows the design beam trajectory and the horizontal and vertical components $x(s), y(s)$ describe the particle's motion relative to the design path.

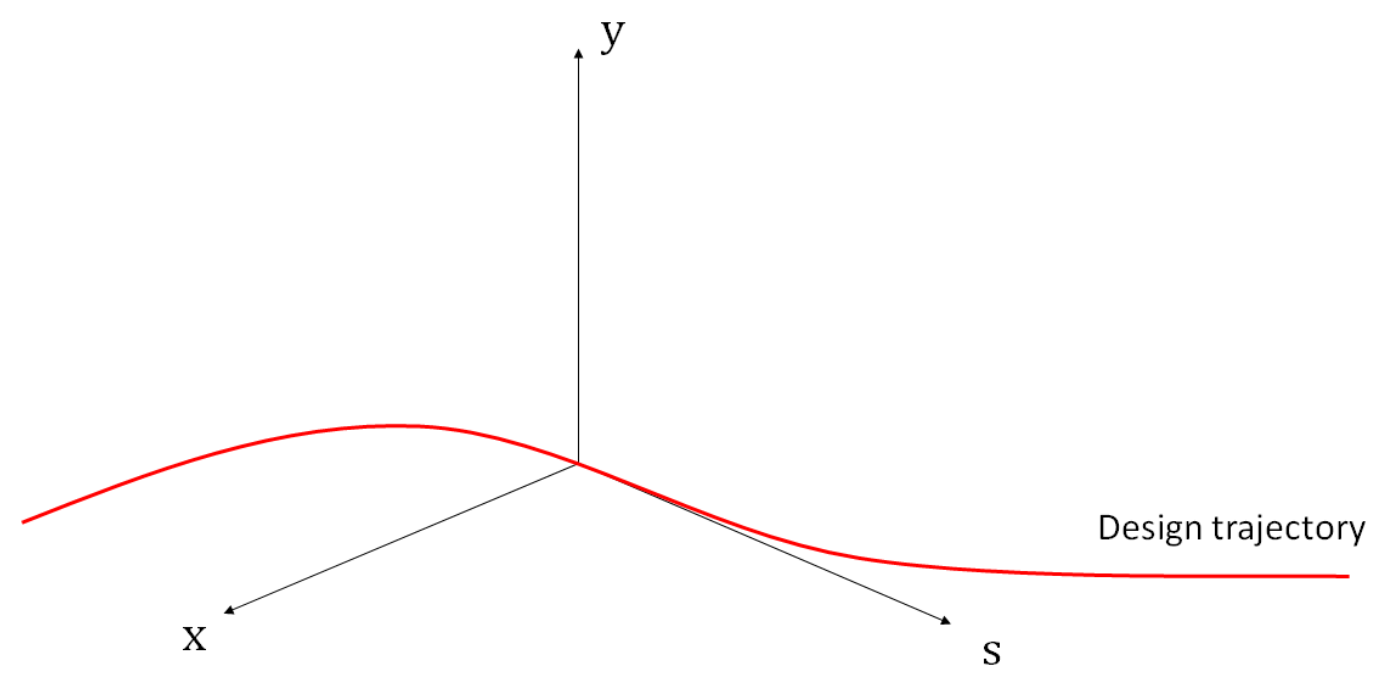

FIG. 23: System of coordinates where $s$ is aligned to the design beam trajectory.

\subsubsection{TRANSVERSE MOTION}

\section{Linear optics}

The transverse magnetic field is

$$
\mathbf{B}=B_{x} \hat{x}+B_{y} \hat{y}
$$

The multipolar expansion in complex notation is

$$
B_{x}+i B_{y}=B_{0} \sum_{n=0}^{\infty}\left(a_{n}+i b_{n}\right)\left(\frac{x+i y}{a}\right)^{n},
$$

where the index $n=0$ corresponds to dipole, $n=1$ to quadrupole, etc. The coefficients $b_{n}$ and $a_{n}$ are called the normal and skew multipoles, correspondingly. The solution of the 
equation of motion of a particle that passes along each element can be more conveniently described through their corresponding transport matrix.

The stable transverse motion of particles is described in terms of oscillations around the design trajectory. This type of motion is also known as betatron motion [34], since it was in a betatron machine where it was first observed. The equations of motion in the horizontal and vertical planes are respectively

$$
\begin{aligned}
x^{\prime \prime}+K_{x} x & =0, & K_{x} & \equiv \frac{e}{p} \frac{\partial B}{\partial x}+\frac{1}{\rho^{2}}, \\
y^{\prime \prime}+K_{y} y & =0, & K_{y} & \equiv-\frac{e}{p} \frac{\partial B}{\partial x},
\end{aligned}
$$

where $x$ and $y$ are the horizontal and vertical displacements relative to the ideal trajectory and no vertical bending, i.e. the reference trajectory is assumed to lie in the $x s$ plane, with $\rho$ is the radius of curvature due to a vertical static magnetic field. $K_{x}(s)$ and $K_{y}(s)$ are both functions of $s$ and carry the description of accelerator lattice through the magnetic fields along the machine. The solutions to Eqs. (54) can be expressed as [34]

$$
\begin{aligned}
x & =A \sqrt{\beta} \cos (\psi+\delta) \\
x^{\prime} & =-\frac{A}{\sqrt{\beta}}[\alpha \cos (\psi+\delta)+\sin (\psi+\delta)],
\end{aligned}
$$

with $A$ and $\delta$ constants of integration and $\alpha \equiv \frac{1}{2} d \beta(s) / d s$. Writing the constant $A$ in terms of the coordinates gives

$$
A^{2}=\gamma x^{2}+2 \alpha x x^{\prime}+\beta x^{2}
$$

with $\gamma \equiv\left(1+\alpha^{2}\right) / \beta$. Equation (57) is known as the Courant-Snyder invariant. The parameters $\alpha, \beta$ and $\gamma$ are known as the Twiss parameters and are used to describe the accelerator machine at any position $s$. A similar set of solutions are found for the $y$ motion.

Since particle accelerators are in a general sense modular machines, it is advantageous to use a matrix formalism when describing the optics of the machine. For example, in advancing the horizontal coordinates from a location $s_{1}$ to a location $s_{2}$, we can use a transformation

$$
\left[\begin{array}{l}
x\left(s_{2}\right) \\
x\left(s_{2}\right)^{\prime}
\end{array}\right]=\mathbb{M}\left(s_{1}, s_{2}\right) \cdot\left[\begin{array}{l}
x\left(s_{1}\right) \\
x\left(s_{1}\right)^{\prime}
\end{array}\right]_{1}
$$

where the transport matrix is 35

$$
\mathbb{M}\left(s_{1}, s_{2}\right)=\left[\begin{array}{cc}
\sqrt{\frac{\beta_{2}}{\beta_{1}}}\left(\cos \Delta \psi+\alpha_{1} \sin \Delta \psi\right) & \sqrt{\beta_{1} \beta_{2} \sin \Delta \psi} \\
-\frac{1+\alpha_{1} \alpha_{2}}{\sqrt{\beta_{1} \beta_{2}}} \sin \Delta \psi+\frac{\alpha_{1}-\alpha_{2}}{\sqrt{\beta_{1} \beta_{2}}} \cos \Delta \psi & \sqrt{\frac{\beta_{1}}{\beta_{2}}}\left(\cos \Delta \psi-\alpha_{2} \sin \Delta \psi\right.
\end{array}\right]
$$


and depends on the Twiss parameters at the $s_{1}$ and $s_{2}$ locations in the accelerator. The phase advance $\Delta \psi$ is given by

$$
\Delta \psi=\tan ^{-1}\left(\frac{m_{12}}{\beta_{1} m_{11}-\alpha_{1} m_{12}}\right),
$$

with $m_{i j}$ are elements of $\mathbb{M}$.

For a circular machine, the lattice repeats itself every circumference $C$, and for one turn, the matrix $\mathbb{M}$ reduces to

$$
\mathbb{M}=\mathbb{I} \cos \psi_{C}+\mathbb{J} \sin \psi_{C},
$$

with $\mathbb{I}$ the identity matrix and

$$
\mathbb{J}=\left[\begin{array}{cc}
\alpha & \beta \\
-\gamma & -\alpha
\end{array}\right] \text {. }
$$

A stability condition for the beam motion is then [35]

$$
\left|\frac{1}{2} \operatorname{tr} \mathbb{M}\right| \leq 1 .
$$

Similarly, if the matrix elements of $\mathbb{M}$ are known, the Twiss parameters from $s_{1}$ to $s_{2}$ transform according to

$$
\left[\begin{array}{l}
\beta_{2} \\
\alpha_{2} \\
\gamma_{2}
\end{array}\right]_{2}=\left[\begin{array}{ccc}
m_{11}^{2} & -2 m_{11} m_{22} & m_{12}^{2} \\
-m_{11} m_{21} & m_{11} m_{22}+m_{12} m_{21} & -m_{12} m_{22} \\
m_{21}^{2} & -2 m_{21} m_{22} & m_{22}^{2}
\end{array}\right] \cdot\left[\begin{array}{c}
\beta_{1} \\
\alpha_{1} \\
\gamma_{1}
\end{array}\right],
$$

An important parameter is the number of betatron oscillations per turn, known as the betatron tune $\nu$

$$
\nu \equiv \frac{\Delta \psi_{C}}{2 \pi}=\frac{1}{2 \pi} \oint \frac{d s}{\beta},
$$

where we are interested in the fractional number.

\section{Momentum dispersion}

In general, particles will differ on momentum with respect to the design particle by a momentum deviation

$$
\delta \equiv \frac{\Delta p}{p_{0}} \ll 1 .
$$

This offset introduces an inhomogeneous term in the equations of motion

$$
x^{\prime \prime}+K_{x} x=\frac{\delta}{\rho},
$$

which is called the dispersion term. 


\section{Beam emittance}

Because of differing momenta of particles in the beam, it occupies a certain area at any location $s$, see Fig. 24.

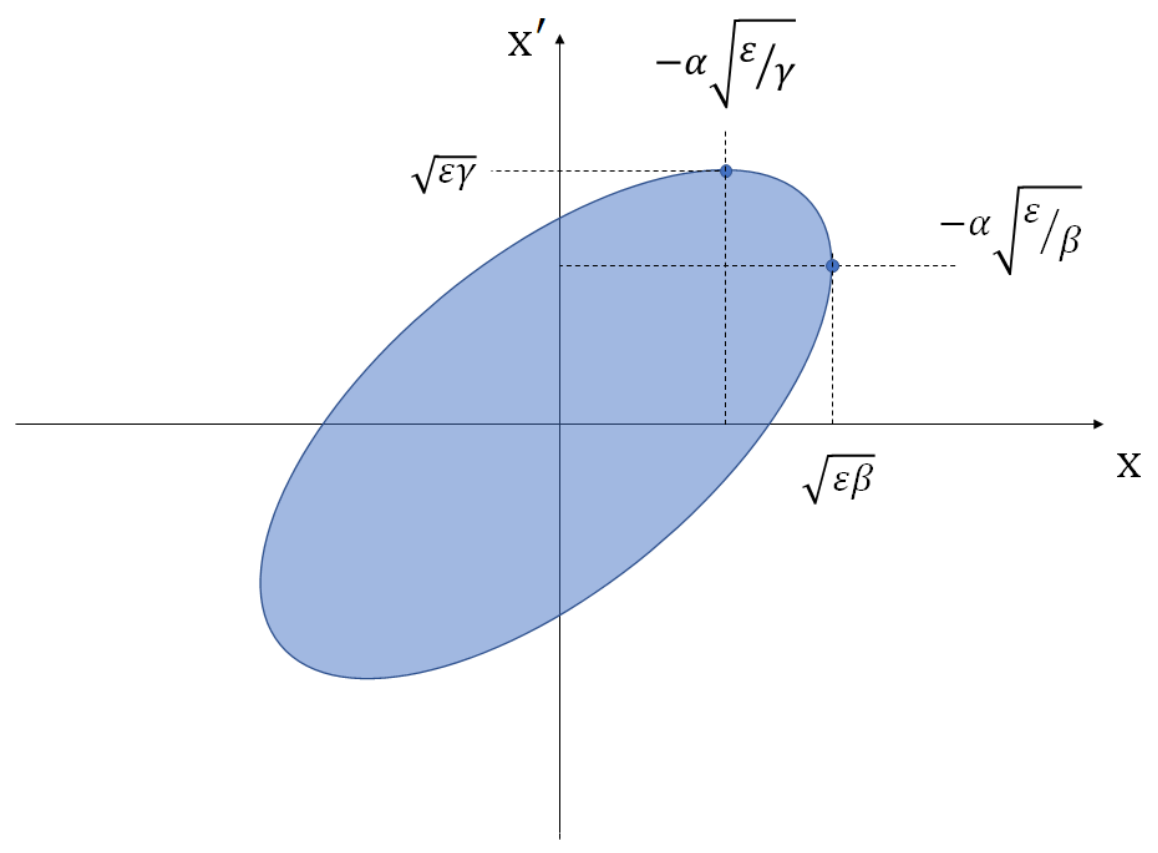

FIG. 24: The beam "paints" an area in phase space, characterized by the Twiss parameters.

The beam emittance is defined as the area in phase space $x x^{\prime}$ that circumscribes a certain fraction $F$ of the beam

$$
\epsilon=-\frac{2 \pi \sigma^{2}}{\beta} \ln (1-F),
$$

where $\sigma$ is usually chosen to correspond to the rms $\sigma$ of a 2D Gaussian distribution

$$
f(x)=\frac{1}{\sqrt{2 \pi} \sigma} \exp \left(-\frac{x^{2}}{2 \sigma^{2}}\right) .
$$

The beam emittance is an important parameter that characterizes the brightness of the beam and it can be used to calculate the beam size $\sigma(s)$ at a location $s$ in the ring using [35]

$$
\sigma(s)=\sqrt{\epsilon \beta(s)+\left[\eta(s) \frac{\Delta p}{p}\right]^{2}} .
$$

The emittance by itself is not an invariant during acceleration of particles, but it scales with the relativistic factor. It is therefore convenient to talk about the normalized beam emittance

$$
\epsilon^{N}=\beta \gamma \epsilon_{g}
$$


with $\epsilon_{g}$ the geometric emittance.

\subsubsection{LONGITUDINAL MOTION}

The stable longitudinal motion of particles around the design energy is described in terms of small oscillations known as synchrotron oscillations. The synchrotron oscillation tune is given by 35

$$
\nu_{s}=\sqrt{\frac{h \eta}{2 \pi \beta^{2} E_{\mathrm{b}}} e V_{0} \cos \phi_{s}},
$$

where $h=f_{\mathrm{rf}} / f_{\mathrm{rev}}$ is the harmonic number, $\eta$ is the phase slip factor, $V_{0}$ is the rf accelerating voltage, $\phi_{s}$ is the synchrotron phase and $E_{\mathrm{b}}$ is the particle energy.

\subsection{LUMINOSITY}

Because of the time-varying nature of the rf accelerating fields, the beams in a collider are not a continuous "filament" of particles but rather a train of bunches of particles. In a particle collider, bunches traveling in opposing directions are brought together into collision at a point where detectors are located [36].

Besides the energy of the beams, a parameter of interest in a particle collider is the luminosity. The luminosity is defined as the rate of collisions $R$ per unit of interaction cross section $\sigma_{\text {int }}$ :

$$
L \equiv \frac{R}{\sigma_{\mathrm{int}}},
$$

The rate of collisions depends on the geometry of the beams, their density and energy. A very simple example is that of a beam going through a fixed target. In this situation, the rate of collisions is proportional to the number of particles per unit time going into the target, the density of particles in the target and its depth. The proportionality factor is called the cross section of the particular interaction and has units of area.

We will now present a general expression of a collider luminosity [37], where the target is not fixed but rather a beam traveling in opposite direction. Consider the collision of two bunches with particle densities $\rho_{1}(x, y, z)$ and $\rho_{2}(x, y, z)$ respectively. The interaction cross section between any two particles in opposing bunches is $\sigma_{\text {int }}$. The probability of a single particle in bunch 1 to interact with any particle in bunch 2 is given by $\sigma_{\text {int }} /$ (bunch cross section), times the total number of particles in bunch 2 ,

$$
\left(\frac{\sigma_{\text {int }}}{d x d y}\right) \cdot \rho_{2}(x, y, z) d x d y d z .
$$


The total probability of any interaction between the two bunches is obtained by multiplying by the total number of particles in bunch 1 ,

$$
\left(\frac{\sigma_{\text {int }}}{d x d y}\right) \cdot \rho_{2}(x, y, z) d x d y d z \cdot \rho_{1}(x, y, z) d x d y d z
$$

The rate of collisions is given by the total number of collisions over the time it takes both bunches to cross each other, this can be accounted for with a kinematic factor ${ }^{1}$ $2 \cos ^{2}\left(\phi_{\text {cross }} / 2\right)$. If bunches collide at a frequency $f$, then the collision rate is

$$
R=\sigma_{\mathrm{int}} 2 c f \cos ^{2}\left(\phi_{\mathrm{cross}} / 2\right) \int_{V} \int_{t} \rho_{1}(x, y, z) \cdot \rho_{2}(x, y, z) d x d y d z .
$$

From the luminosity definition given by Eq. (73), we obtain an expression for the luminosity

$$
L=2 c f \cos ^{2}\left(\phi_{\text {cross }} / 2\right) \int_{V} \int_{t} \rho_{1}(x, y, z) \rho_{2}(x, y, z) d x d y d z d t .
$$

Note from the definition, Eq. (73), that the collision rate can be separated as the product of two terms: the interaction cross section and the luminosity. This distinction is important because the interaction cross section $\sigma_{\text {int }}$ is a quantity that depends entirely on the fundamental physics processes behind the collision of particles, i.e. the dynamics of quarks and gluons in the collision between two nucleons. From a high-energy physics user perspective, the luminosity can also be used to normalize experimental measurements. The luminosity $L$ on the other hand, depends only on machine parameters, and as such, can be engineered to a design value, mainly limited by the technological capabilities. In particular, the number of particles in the bunches relates to the beam intensity, or the beam current; the frequency of collisions is determined by the selected rf systems; the cross section of the bunches is related to the beam injector capabilities, the beam emittance and optical beta function.

\footnotetext{
${ }^{1}$ In general, the kinematic factor of the collision of two beams can be calculated from the scalar product of the corresponding 4 -velocities of the beams $\mathbf{u}_{1}=\left(1, \mathbf{v}_{1}\right)$ and $\mathbf{u}_{2}=\left(1, \mathbf{v}_{2}\right)$ :

$$
\begin{aligned}
\mathbf{u}_{1} \cdot \mathbf{u}_{2} & =g^{\mu \nu} v_{\mu} v_{\nu} \\
& =1-\mathbf{v}_{1} \cdot \mathbf{v}_{2}
\end{aligned}
$$
}

If the beams move such that $\mathbf{v}_{1}=-\mathbf{v}_{2}$ and there is a crossing angle $\phi_{\text {cross }}$ between them, then the kinematic factor is

$$
\begin{aligned}
1-\mathbf{v}_{1} \cdot \mathbf{v}_{2} & =1-v_{1} v_{2} \cos \phi_{\text {cross }} \\
& =1+\beta^{2} \cos \phi_{\text {cross }} \\
& =1+\beta^{2}\left[2 \cos ^{2}\left(\phi_{\text {cross }} / 2\right)-1\right] \\
& \approx 2 \cos ^{2}\left(\phi_{\text {cross }} / 2\right)
\end{aligned}
$$

in the limit where $\beta \simeq 1$. 
The luminosity is expressed in units of (area $\cdot$ time $)^{-1}$ and typical luminosity values in colliders like LHC, RHIC and KEK are in the $10^{30} \mathrm{~cm}^{-2} \mathrm{~s}^{-1}$ range. Although this sounds large, the interaction cross sections are typically very small quantities, on the order of physical nuclear cross sections of $10^{-24} \mathrm{~cm}^{2}$.

What I have referred to so far is also known as the instantaneous luminosity of collisions. The integrated luminosity over time has units of inverse area and gives the overall collisions produced in a machine over a certain period of time. It is usually used to predict the physics output of a collider over a certain time period of operations.

\subsubsection{LUMINOSITY OF HEAD-ON COLLISION}

We will now consider the collision of two perfectly aligned bunches, where we assume for simplicity that their distributions are uniformly cylindrical with transverse cross section $A$, where each bunch is formed by $n_{1}$ and $n_{2}$ particles correspondingly, as shown in Fig. 25. If

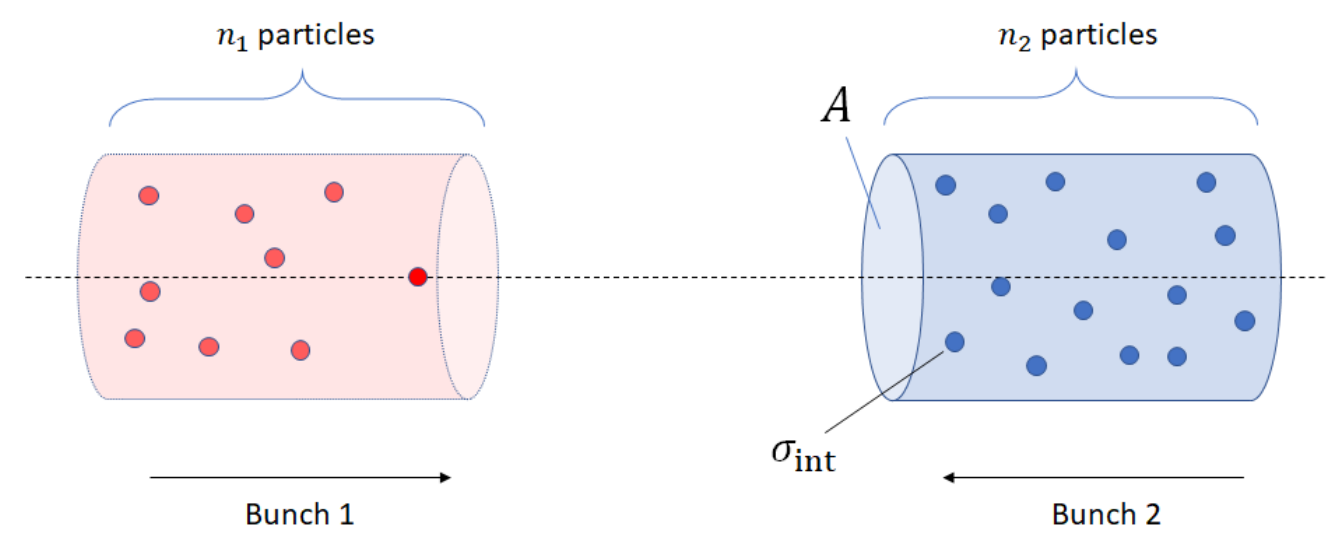

FIG. 25: Idealized collision of two cylindric bunches with $n_{1}$ and $n_{2}$ particles and interaction cross section $\sigma_{\text {int }}$.

the interaction cross section between any 2 particles in opposite beams is $\sigma_{\text {int }}$, the probability of a particle of beam 1 to interact with any particle in beam 2 is $n_{2} \sigma_{\text {int }} / A$. Since there are also $n_{1}$ particles in bunch 1 , then the number of interactions when the bunches cross each other is $n_{1} n_{2} \sigma_{\text {int }} / \mathrm{A}$. If in addition the two beams are formed by many identical bunches, and the frequency of collisions is $f$, then the rate of collisions $R$ is

$$
R=f \frac{n_{1} n_{2}}{A} \sigma_{\mathrm{int}},
$$

and the luminosity in this case becomes

$$
L=f \frac{n_{1} n_{2}}{A} .
$$


If instead we use bunches with symmetric Gaussian transverse profiles with standard deviation $\sigma_{0}$, the luminosity becomes

$$
L=f \frac{n_{1} n_{2}}{4 \pi \sigma_{0}^{2}}
$$

as will be shown in following sections.

\subsubsection{GENERAL EXPRESSION FOR THE LUMINOSITY}

We now discuss a more realistic collision scenario, where in the $x z$ plane, the beams cross each other at a finite angle $\alpha_{x}$, and there may be a small offset $\Delta_{x}$ between the beams at the interaction point in $z=0$. The geometry of this collision is presented in Fig. 26. The situation is similar in the plane $y z$, where the beams cross each other at an angle $\alpha_{y}$ and there may be an offset $\Delta_{y}$ at the interaction point. The trajectories of the beams are given

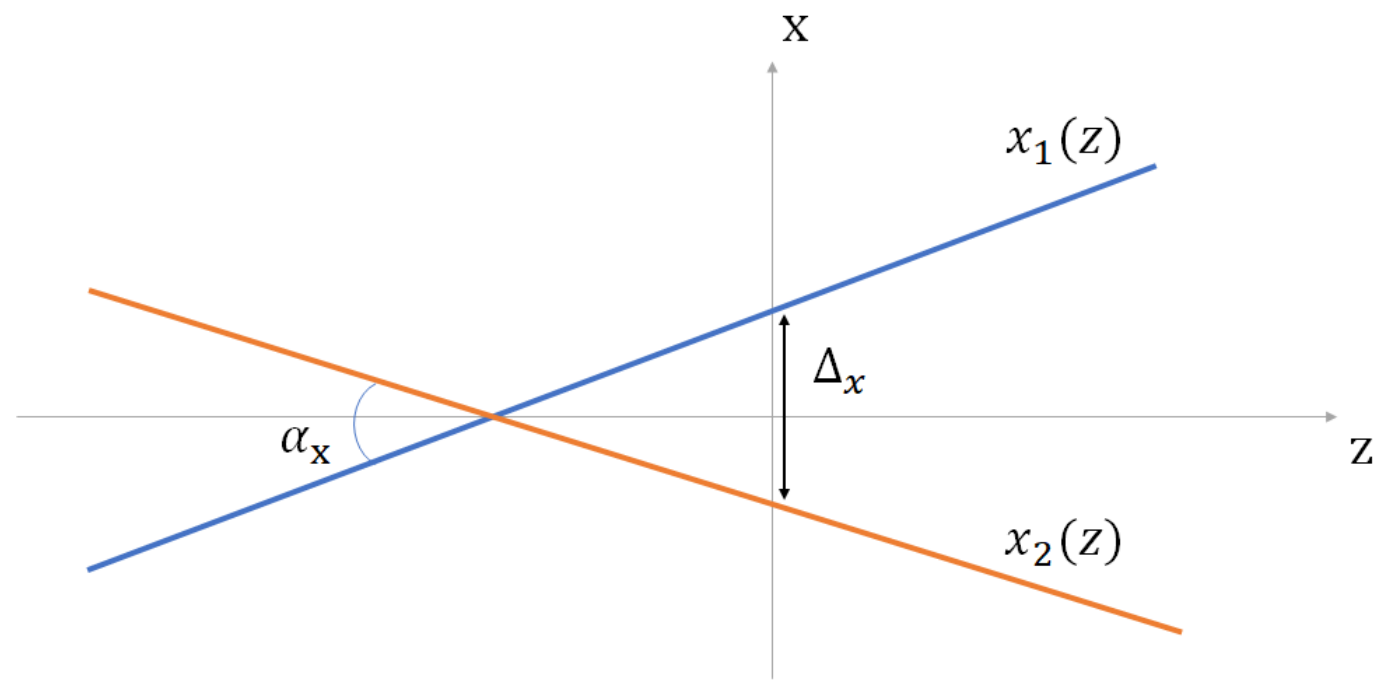

FIG. 26: Beams crossing in the plane $x z$ with a crossing angle $\alpha_{x}$ and a transverse offset $\Delta_{x}$ between beams at the IP.

by $x_{1}(z), y_{1}(z), x_{2}(z)$ and $y_{2}(z)$. In explicit form, the trajectories are given by

$$
\begin{array}{cc}
x_{1}(z)=\frac{\alpha_{x}}{2} z+\frac{\Delta_{x}}{2}, & y_{1}(z)=\frac{\alpha_{y}}{2} z+\frac{\Delta_{y}}{2} \\
x_{2}(z)=-\left(\frac{\alpha_{x}}{2} z+\frac{\Delta_{x}}{2}\right), & y_{2}(z)=-\left(\frac{\alpha_{y}}{2} z+\frac{\Delta_{y}}{2}\right) .
\end{array}
$$

Let us now make assumptions on the colliding bunches. Assume there are $n$ particles in a bunch, and their density follows Gaussian distributions in the $x, y$ and $z$ coordinates, with 
corresponding standard deviations $\sigma_{x}, \sigma_{y}$ and $\sigma_{z}$, then the density of particles is

$$
\rho(x, y, z)=\frac{n}{(2 \pi)^{3 / 2} \sigma_{x} \sigma_{y} \sigma_{z}} \exp \left\{-\frac{\left(x-\mu_{x}\right)^{2}}{2 \sigma_{x}^{2}}\right\} \exp \left\{-\frac{\left(y-\mu_{y}\right)^{2}}{2 \sigma_{y}^{2}}\right\} \exp \left\{-\frac{\left(z-\mu_{z}\right)^{2}}{2 \sigma_{z}^{2}}\right\} .
$$

Now the bunch centers, given by $\mu_{x}, \mu_{y}$ and $\mu_{z}$, travel along the beam trajectories given by Eqs. (81), then the particle distributions corresponding to each bunch are

$$
\begin{aligned}
& \rho_{1}(x, y, z)=\frac{n_{1}}{(2 \pi)^{3 / 2} \sigma_{x} \sigma_{y} \sigma_{z}} \exp \left\{-\frac{\left[x-x_{1}(z)\right]^{2}}{2 \sigma_{x}^{2}}-\frac{\left[y-y_{1}(z)\right]^{2}}{2 \sigma_{y}^{2}}-\frac{[z-c t]^{2}}{2 \sigma_{z}^{2}}\right\}, \\
& \rho_{2}(x, y, z)=\frac{n_{2}}{(2 \pi)^{3 / 2} \sigma_{x} \sigma_{y} \sigma_{z}} \exp \left\{-\frac{\left[x-x_{2}(z)\right]^{2}}{2 \sigma_{x}^{2}}-\frac{\left[y-y_{2}(z)\right]^{2}}{2 \sigma_{y}^{2}}-\frac{[z+c t]^{2}}{2 \sigma_{z}^{2}}\right\} .
\end{aligned}
$$

We now calculate the luminosity using Eq. (77). Substitution of the bunch densities given by Eqs. 83 and 84 gives

$$
\begin{array}{r}
L=2 c f \cos ^{2}\left(\phi_{\text {cross }} / 2\right) \int_{V} \int_{t} \frac{n_{1} n_{2}}{(2 \pi)^{3} \sigma_{x}^{2} \sigma_{y}^{2} \sigma_{z}^{2}} \exp \left\{-\frac{\left[x-x_{1}(z)\right]^{2}}{2 \sigma_{x}^{2}}-\frac{\left[y-y_{1}(z)\right]^{2}}{2 \sigma_{y}^{2}}-\frac{[z-c t]^{2}}{2 \sigma_{z}^{2}}\right\} \\
\cdot \exp \left\{-\frac{\left[x-x_{2}(z)\right]^{2}}{2 \sigma_{x}^{2}}-\frac{\left[y-y_{2}(z)\right]^{2}}{2 \sigma_{y}^{2}}-\frac{[z+c t]^{2}}{2 \sigma_{z}^{2}}\right\} d x d y d z d t,
\end{array}
$$

to get

$$
\begin{gathered}
L=c f \cos ^{2}\left(\phi_{\operatorname{cross}} / 2\right) \frac{n_{1} n_{2}}{(2 \pi)^{3} \sigma_{z}^{2}} \int \exp \left[-\frac{c^{2} t^{2}}{\sigma_{z}^{2}}\right] d t \\
\cdot \frac{1}{\sigma_{x}} \int \exp \left[-\frac{x^{2}}{\sigma_{x}^{2}}\right] d x \cdot \frac{1}{\sigma_{y}} \int \exp \left[-\frac{y^{2}}{\sigma_{y}^{2}}\right] d y \cdot \frac{1}{\sigma_{x} \sigma_{y}} \int \exp \left[-\frac{x_{1}^{2}+x_{2}^{2}}{2 \sigma_{x}^{2}}-\frac{y_{1}^{2}+y_{2}^{2}}{2 \sigma_{y}^{2}}-\frac{z^{2}}{\sigma_{z}^{2}}\right] d z
\end{gathered}
$$

and

$$
L=2 f \cos ^{2}\left(\phi_{\mathrm{cross}} / 2\right) \frac{n_{1} n_{2}}{4 \pi \sqrt{\pi} \sigma_{z}} \int\left\{\frac{1}{\sigma_{x} \sigma_{y}} \exp \left[-\frac{x_{1}^{2}+x_{2}^{2}}{2 \sigma_{x}^{2}}-\frac{y_{1}^{2}+y_{2}^{2}}{2 \sigma_{y}^{2}}-\frac{z^{2}}{\sigma_{z}^{2}}\right]\right\} d z
$$

Substitute now the explicit centroid trajectories given by Eqs. (81), together with the $\sigma_{x}(z)$ and $\sigma_{y}(z)$,

$$
\begin{aligned}
& \sigma_{x}^{2}(z)=\frac{\epsilon \beta_{x}^{*}}{6 \pi \gamma}\left[1+\left(\frac{z-z_{x 0}}{\beta_{x}^{*}}\right)^{2}\right], \\
& \sigma_{y}^{2}(z)=\frac{\epsilon \beta_{y}^{*}}{6 \pi \gamma}\left[1+\left(\frac{z-z_{y 0}}{\beta_{y}^{*}}\right)^{2}\right],
\end{aligned}
$$

where the betatron function is assumed to have a parabolic dependence around the interaction point. We arrive at a general expression for the luminosity of two Gaussian bunches 
with finite crossing angles and offset from the IP

$$
L=\frac{f n_{1} n_{2}}{4 \pi \sigma_{0}^{2}}\left(\frac{\beta^{*}}{\sqrt{\beta_{x}^{*} \beta_{y}^{*}}}\right) \frac{\cos ^{2}\left(\phi_{\mathrm{cross}} / 2\right)}{\sqrt{\pi} \sigma_{z}} \int_{-\infty}^{\infty} \frac{\exp \left\{-\left[\frac{\Delta_{x}+\alpha_{x} z}{2 \sigma_{x}(z)}\right]^{2}+\left[\frac{\Delta_{y}+\alpha_{y} z}{2 \sigma_{y}(z)}\right]^{2}\right\}}{\sqrt{\left[1+\left(\frac{z-z_{x 0}}{\beta_{x}^{*}}\right)^{2}\right]\left[1+\left(\frac{z-z_{y} 0}{\beta_{y}^{*}}\right)^{2}\right]}} e^{-z^{2} / \sigma_{z}^{2}} d z
$$

where $\beta^{*}$ are the design values of $\beta$ at the interaction point and $\sigma_{0}$ is the design value of the $r m s$ beam size, given by the $95 \%$ emittance. Note that for an ideal case of 2 Gaussian bunches with $n_{1}$ and $n_{2}$ particles each, with transverse dimensions $\sigma_{x}=\sigma_{y}=\sigma_{0}$, with no crossing angle and aligned to each other, the luminosity takes a similar form to the one in simplified collision of cylindrical bunches.

$$
L_{0}=\frac{f n_{1} n_{2}}{4 \pi \sigma_{0}^{2}}
$$

\subsubsection{LUMINOSITY WITH BEAM CROSSING ANGLE}

The particular case we are interested in this dissertation if that of a collision of two beams with a crossing angle, specifically in the horizontal plane, as this is the scenario being designed for JLEIC. A crossing angle might not seem ideal from the point of view of luminosity but it also has various advantages. It ensures a rapid beam separations, reducing parasitic collisions away from the interaction point, it also allows more space for interaction region magnets. Parting from the general expression of luminosity in Eq. (87), the case we are interested is that of $\Delta_{x}=\Delta_{y}=0$, no offset and $\alpha_{y}=0$, no vertical crossing angle,

$$
L=\frac{f n_{1} n_{2}}{4 \pi \sigma_{0}^{2}} \cdot\left(\frac{\beta^{*}}{\sqrt{\beta_{x}^{*} \beta_{y}^{*}}}\right) \frac{\cos ^{2}\left(\phi_{\text {cross }} / 2\right)}{\sqrt{\pi} \sigma_{z}} \int_{-\infty}^{\infty} \frac{\exp \left\{-\left[\frac{\alpha_{x} z}{2 \sigma_{x}(z)}\right]^{2}-\frac{z^{2}}{\sigma_{z}^{2}}\right\}}{\sqrt{\left[1+\left(\frac{z-z_{x 0}}{\beta_{x}^{*}}\right)^{2}\right]\left[1+\left(\frac{z-z_{y 0}}{\beta_{y}^{*}}\right)^{2}\right]}} d z
$$

reduces to

$$
L=L_{0} \cdot \frac{\cos ^{2}\left(\phi_{\text {cross }} / 2\right)}{\sqrt{\pi} \sigma_{z}} \int_{-\infty}^{\infty} \exp \left\{-\frac{\alpha^{2} z^{2}}{4 \sigma_{0}^{2}}\right\} \exp \left\{-\frac{z^{2}}{\sigma_{z}^{2}}\right\} d z
$$


which can be evaluated straightforward using a change of variable,

$$
\begin{aligned}
L & =L_{0} \cdot \frac{\cos ^{2}\left(\phi_{\text {cross }} / 2\right)}{\sqrt{\pi} \sigma_{z}} \int_{-\infty}^{\infty} \exp \left\{-\frac{\alpha^{2} z^{2}}{4 \sigma_{0}^{2}}-\frac{z^{2}}{\sigma_{z}^{2}}\right\} d z \\
& =L_{0} \cdot \frac{\cos ^{2}\left(\phi_{\text {cross }} / 2\right)}{\sqrt{\pi} \sigma_{z}} \int_{-\infty}^{\infty} \exp \left\{-\frac{1}{\sigma_{z}^{2}}\left[1+\left(\frac{\alpha \sigma_{z}}{2 \sigma_{0}}\right)^{2}\right] z^{2}\right\} d z \\
& =L_{0} \cdot \frac{\cos ^{2}\left(\phi_{\text {cross }} / 2\right)}{\sqrt{1+\left(\frac{\alpha \sigma_{z}}{2 \sigma_{0}}\right)^{2}}} \frac{\sqrt{1+\left(\frac{\alpha \sigma_{z}}{2 \sigma_{0}}\right)^{2}}}{\sqrt{\pi} \sigma_{z}} \int_{-\infty}^{\infty} \exp \left\{-\frac{\left[1+\left(\frac{\alpha \sigma_{z}}{2 \sigma_{0}}\right)^{2}\right] z^{2}}{\sigma_{z}^{2}}\right\} d z \\
& =L_{0} \cdot \frac{\cos ^{2}\left(\phi_{\text {cross }} / 2\right)}{\sqrt{1+\left(\frac{\alpha \sigma_{z}}{2 \sigma_{0}}\right)^{2}}} \frac{1}{\sqrt{\pi} z_{r m s}} \int_{-\infty}^{\infty} \exp \left\{-\frac{z^{2}}{z_{r m s}^{2}}\right\} d z
\end{aligned}
$$

where

$$
z_{r m s}=\frac{\sigma_{z}}{\sqrt{1+\left(\frac{\alpha \sigma_{z}}{2 \sigma_{0}}\right)^{2}}},
$$

determines the luminous region in the case of long bunches.

We arrive now at the luminosity produced by 2 (short) Gaussian bunches colliding at a crossing angle $\phi_{\text {cross }}$

$$
L=L_{0} \cdot \frac{\cos ^{2}\left(\frac{\phi_{\text {cross }}}{2}\right)}{\sqrt{1+\left(\frac{\phi_{\text {cross }} \sigma_{z}}{2 \sigma_{0}}\right)^{2}}} .
$$

For small crossing angles in the order of mrad, the kinematic term $\cos ^{2}\left(\phi_{\text {cross }} / 2\right) \approx 1$, but the luminosity reduction factor [3]

$$
R_{\Theta}=\frac{1}{\sqrt{1+\Theta^{2}}}
$$

is in general $R_{\Theta} \leq 1$. The parameter $\Theta$ is known as the Piwinski angle,

$$
\Theta \equiv \frac{\phi_{\text {cross }}}{2} \frac{\sigma_{z}}{\sigma_{x}^{*}}
$$

and roughly describes the aspect ratio of the bunches at the collision point. In Fig. 27, the luminosity reduction factor $R_{\Theta}$ is plotted as a function of the Piwinski angle.

As it was mentioned before, two unique characteristics of the JLEIC design are the high luminosity requirement, in the order of $10^{34} \mathrm{~cm}^{-2} \mathrm{~s}^{-1}$, and the integration of a full acceptance detector in the interaction region. For this detector, a relatively large crossing angle $\phi_{\text {cross }}=$ 


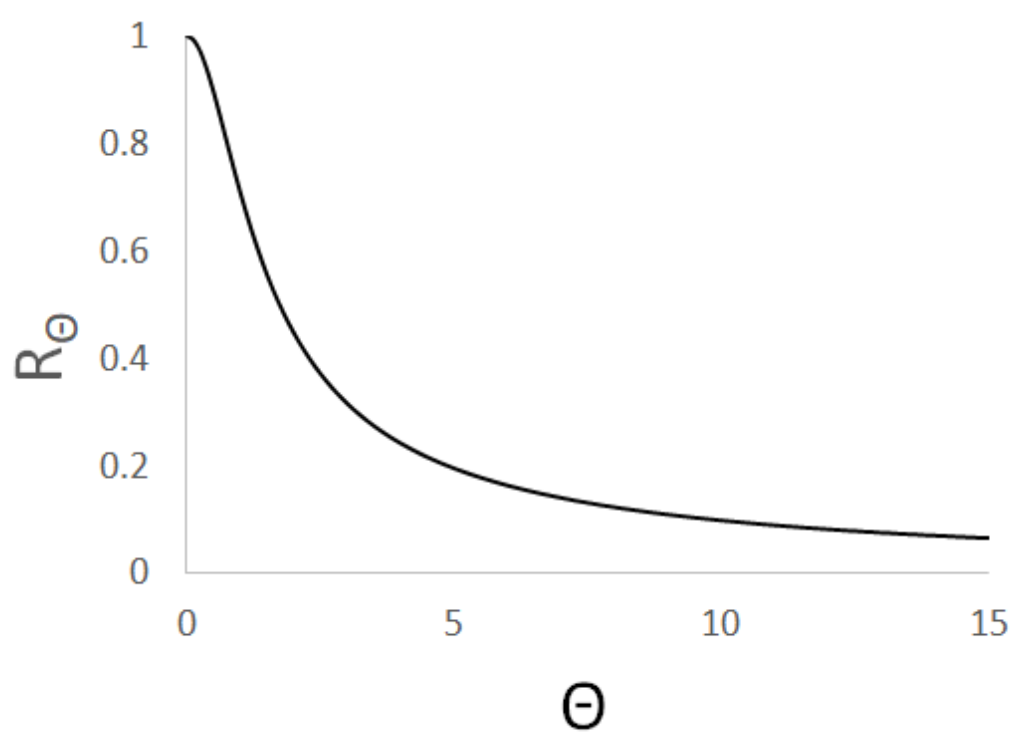

FIG. 27: Qualitative dependence of the luminosity reduction factor with the Piwinski angle.

$50 \mathrm{mrad}$ is required. Using JLEIC ion beam design parameters: $\sigma_{x}^{*}=30 \mu \mathrm{m}, \sigma_{s}=1.2 \mathrm{~cm}$, and the $50 \mathrm{mrad}$ crossing angle, results in a reduction factor $R_{\Theta}=0.11$. This results in an order of magnitude reduction from the available head-on collision luminosity. Table 4 compares the JLEIC luminosity reduction factor with different colliders in other labs.

TABLE 4: Luminosity reduction factor, $R_{\Theta}$, of different particle colliders [3].

\begin{tabular}{|c|c|c|c|c|c|}
\hline Colliders & $\sigma_{z}[\mathrm{~mm}]$ & $\sigma_{x}^{*}[\mu \mathrm{m}]$ & $\phi_{\text {cross }}[\mathrm{mrad}]$ & $\Theta$ & $R_{\Theta}$ \\
\hline DORIS-1 & 10 & 230 & 24 & 0.52 & 0.89 \\
\hline DA $\Phi$ NE & 18 & 700 & 30 & 0.39 & 0.93 \\
\hline KEK-B & 7 & 103 & 22 & 0.75 & 0.8 \\
\hline RHIC & 140 & 177 & \pm 0.5 & 0.20 & 0.98 \\
\hline LHC & 75.5 & 16.6 & 0.285 & 0.65 & 0.84 \\
\hline JLEIC & 12 & 30 & 50 & 10 & 0.1 \\
\hline
\end{tabular}

In particular, note that JLEIC has the largest design crossing angle. Also, RHIC does not have a design crossing angle, but rather operates with crossing angles of $\pm 0.5 \mathrm{mrad}$ due to limited resolution on the BPMs and diurnal orbit motion [3]. 


\subsection{BUNCH CRABBING IN COLLIDERS}

As mentioned in previous section, the effect on luminosity of introducing a crossing angle between the two beams is a geometric reduction factor $R_{\Theta}=\frac{1}{\sqrt{1+\Theta^{2}}}$. Crab cavities can be used to recover a head on collision of the beams, thus effectively making the Piwinski angle $\Theta$ equal zero, and recevering the otherwise lost luminosity. The concept of crabbing was first proposed by Robert Palmer in 1988 for linacs [12] and was soon after adapted for storage rings [13] as well. A time-dependent transverse kick is given to the bunch to tilt it, effectively having a head-on collision at the interaction point [3], see Fig. 28 .

The tilt is produced by setting the rf phase so that a transverse force is applied to the head of the bunch and then in the opposite direction to the tail of the bunch. With the kick given at the right phase advance relative to the interaction point, this method recovers the luminosity lost to the crossing angle. In standard accelerator coordinates, the crab cavity introduces a kick

$$
\begin{aligned}
\Delta p_{x} & =-\frac{\partial H_{\mathrm{crab}}}{\partial x}, \\
& =-\frac{q V_{\mathrm{crab}}}{E_{b}} \cdot \sin \left(\frac{\omega z}{c}+\phi_{s}\right),
\end{aligned}
$$

where $q$ is the charge of the particle and $E_{b}$ its energy, $V_{\text {crab }}$ is the voltage, $\omega$ the angular rf frequency and $\phi_{s}$ the synchronous phase of the crab cavity, $z$ is the longitudinal and $x$ the transverse position of a particle with respect to the bunch center and $H_{\text {crab }}$ is the Hamiltonian describing a thin crab cavity [39]

$$
H_{\mathrm{crab}}=\frac{q V}{E_{b}} \cdot \sin \left(\frac{\omega z}{c}+\phi_{s}\right) \cdot x .
$$

Note that if the phase of the cavity fields is adjusted such that the field is maximum at the bunch center, then the cavity acts as a deflector.

A bunch crabbing scheme can be implemented in two different configurations: a local and global crabbing scheme.

\subsubsection{LOCAL CRABBING}

In the local crabbing scheme, crab cavities are placed at points before and after the interaction point. The idea is to have the first crab cavity produce a tilt on the bunches, such that at the interaction point the two beams collide head-on and thus increase the luminosity. The second crab cavity, located after the interaction point, cancels the initial tilt such as to remove the induced oscillation anywhere else in the ring, canceling any crab 
cavity induced effects. In this sense, crab cavities create a local, phase-dependent bump on the beam orbit. Each beam needs to be tilted by half the crossing angle. In order to produce the correct tilt on the bunch, crab cavities need to be able to produce a transverse voltage

$$
V_{\mathrm{C} 1}=\frac{c E_{\mathrm{b}}}{e 2 \pi f \sqrt{\beta_{x}^{\mathrm{crab}} \beta_{x}^{*}} \sin \left(\Delta \psi_{C C \rightarrow I P}\right)} \cdot \tan \left(\frac{\phi_{\text {cross }}}{2}\right),
$$

where $E_{\mathrm{b}}$ is the beam energy, $\phi_{\text {cross }}$ is the total beam crossing angle, $\beta_{x}^{\text {crab }}$ is the horizontal beta function at the crab cavity location, $\beta_{x}^{*}$ is the horizontal beta function at the IP, $\Delta \psi_{C C \rightarrow I P}$ is the horizontal betatron phase advance from the crab cavity location to the interaction point. Note the crab voltage is minimized if cavities are placed at points where

$$
\Delta \psi_{C C \rightarrow I P}=\frac{n \pi}{2}
$$

for integers $n$. Additionally, the required voltage for the second crab cavity to remove the bunch tilt is

$$
V_{C 2}=-R_{22} V_{C 1}
$$

where $R_{22}$ is the $(2,2)$ transport matrix element between the two crab cavities.

Figure 28 shows a diagram of the local crabbing scheme to recover head-on collisions at the interaction point; electron and ion beams approach the interaction region, when passing through the crab cavities, bunches experiences a transverse field that is zero at the bunch center and opposite at the head and tail. In this example, crab cavities are placed at a distance corresponding to $\Delta \psi_{C C \rightarrow I P}=\pi / 2$ both before and after the interaction point, this is a $\pi$-bump crab crossing, since the induced oscillation corresponds to half of the betatron oscillation. 


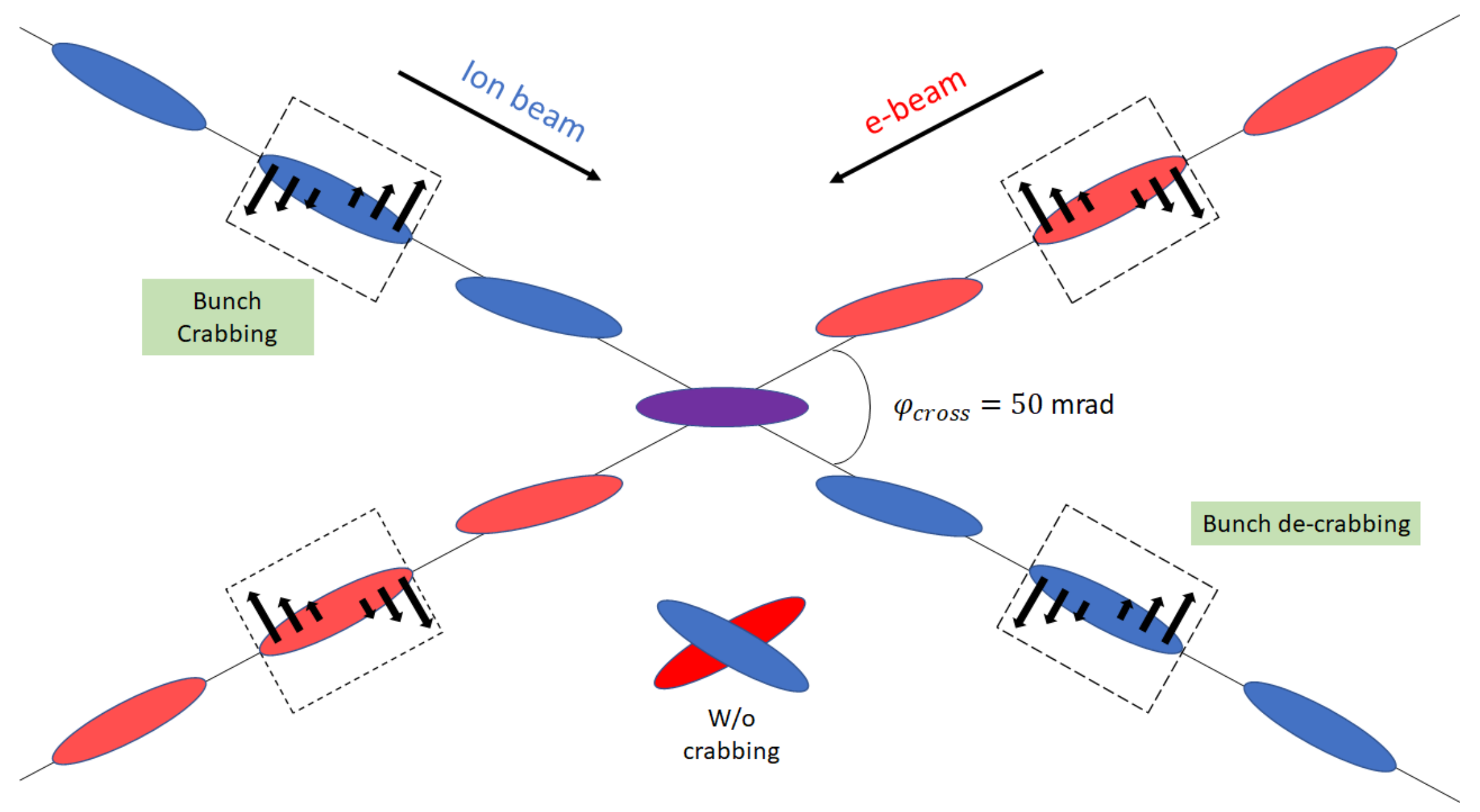

FIG. 28: Local bunch crabbing scheme for a symmetric interaction region of an electron-ion collider. The betatron phase advance between each crab cavity and IP is $\Delta \psi=\pi / 2$. 


\subsubsection{NON-ZERO DISPERSION AT CRAB CAVITY LOCATIONS}

The local crabbing scheme relies on the full cancellation of the transverse kick effects, this involves the cancellation of the energy change due to beam beam interaction. However, if there is dispersion at the crab cavity locations, this energy change may not be completely cancelled. A stability criterion on the tolerable values of $\eta$ and $\eta^{\prime}$ at the crab cavity location is 38

$$
\eta \eta^{\prime} \leq \frac{\sigma_{x}^{*}}{2 \sigma_{s}} \frac{\alpha C}{\phi_{\text {crab }}}
$$

with $\eta, \eta^{\prime}$ the dispersion and its derivative at the crab cavity locations, $\sigma_{x}^{*}$ is the horizontal transverse bunch size at IP, $\sigma_{s}$ is the rms length of the bunch, $\alpha$ is the momentum compaction factor, $C$ is the circumference of the ring and $\phi_{\text {crab }}$ is the crabbing angle.

\subsubsection{GLOBAL CRABBING}

In the global scheme, the crabbing region is effectively extended so that only one crab cavity is needed for the interaction point. The cavity is placed at a location that satisfies

$$
\cos \left(\psi_{C C \rightarrow I P}-\pi \nu_{\beta}\right)=1
$$

where $\nu_{\beta}$ is the betatron tune, in this crabbing scheme, the required voltage can be calculated from

$$
V=\frac{c^{2} E_{b} \tan \left(\frac{\phi_{\text {cross }}}{2}\right)}{q \omega \sqrt{\beta^{*} \beta_{\text {crab }}}} \cdot\left|\frac{2 \sin \left(\pi \nu_{\beta}\right)}{\cos \left(\psi_{C C \rightarrow I P}-\pi \nu_{\beta}\right)}\right|
$$

The bunch will be tilted and an oscillation would be present everywhere around the ring, but at the interaction point it will be head-on. This was the scheme implemented in KEK-B.

For an argument of completeness, we mention that the crabbing of bunches can be implemented in two different ways, a dispersive and deflective mechanism. In the dispersive crabbing, the bunch tilt is produced by the longitudinal field of a with a conventional bunching, accelerating cavity placed in a dispersion $(\eta \neq 0)$ region in the ring. In this case, the required crabbing voltage (assuming the correct phase advance) is given by

$$
V_{\text {disp. }}=\frac{c E_{\mathrm{b}}}{e 2 \pi f \sqrt{\beta_{x}^{\text {crab }} \beta_{x}^{*}} \eta_{x}^{\prime}} \cdot \tan \left(\frac{\phi_{\text {cross }}}{2}\right),
$$

with $\eta_{x}^{\prime}$ the derivative of dispersion at the crab cavity location.

On the other hand, in a deflective cavity approach, the tilt is produced by a specifically designed cavity operating in a transverse mode. The voltage requirement on the dispersive 


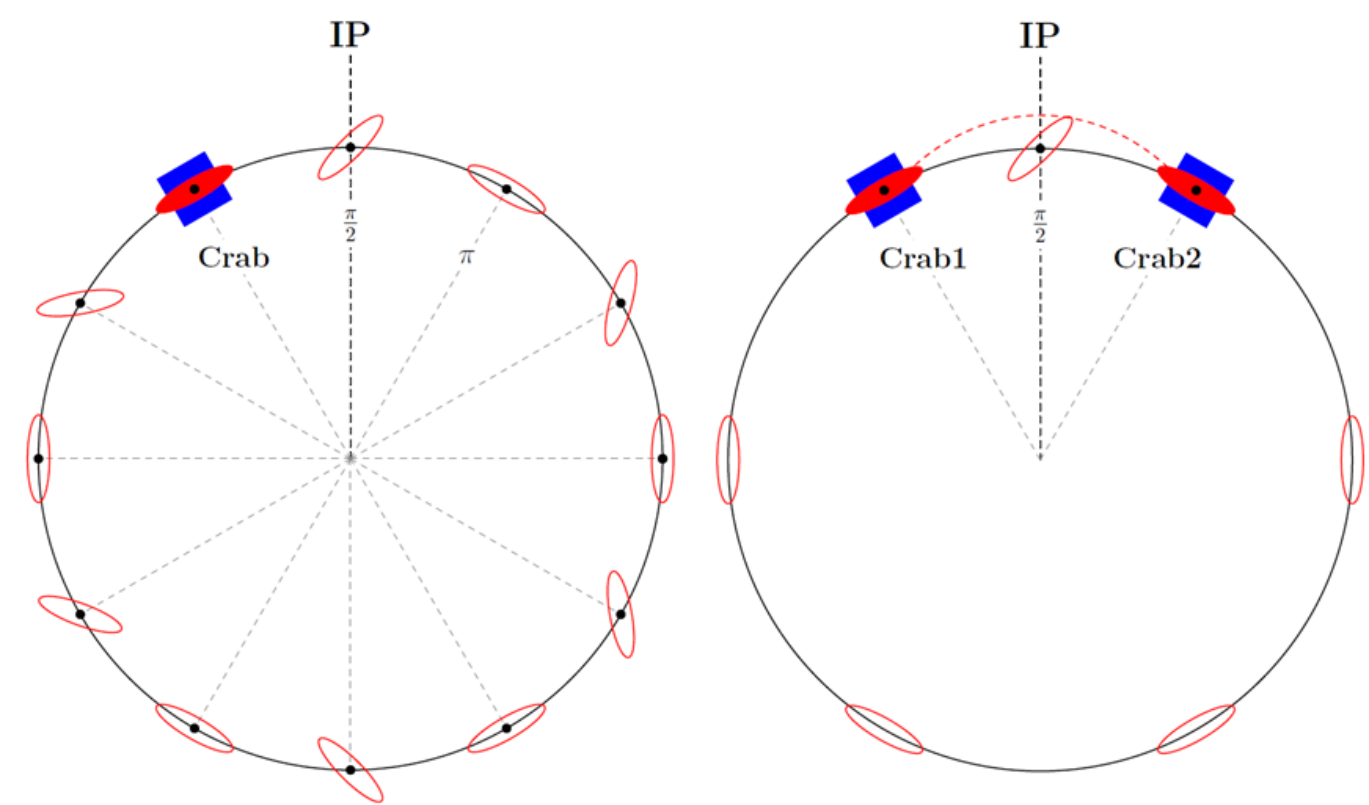

FIG. 29: Global vs. local bunch crabbing scheme. The local crabbing scheme acts as a bump in the beam orbit.

scheme is found to be significantly higher than the deflective scheme: a factor 4.7 in the case of JLEIC ion ring. A deflective crabbing scheme is therefore adopted for JLEIC.

\subsection{JLEIC CRAB CROSSING SCHEME}

This work is a continuation of the previous work on the design of the crabbing scheme for JLEIC [40, 41]. A local crabbing scheme involves the use of crab cavities at both sides of the IR. The upstream cavity imparts the required kick on the beam to recover head-on collisions at the IP. The downstream cavity cancels the initial kick on the beam, effectively constraining the crabbed beam to a region around IP; thus limiting beam effects induced by the transverse kick along the ring. A relative phase advance $\Delta \phi=n \pi$ between the two cavities is required for a full kick cancellation.

The design of the IR includes horizontal and vertical Chromaticity Compensation Blocks (CCB) [42, 43]. Here, sextupole magnets are used to cancel the chromatic kick from the final focusing quadrupoles. These CCB locations have high $\beta$ function values and phase advance suitable for crabbing. Figures 30 and 31 show the CCB optical section of the electron and ion rings, showing the ideal location for crab cavities.

In a local crabbing scheme, the voltage required to tilt the bunch by an angle $\phi_{\text {crab }}$ at 
(1) (3) (2)

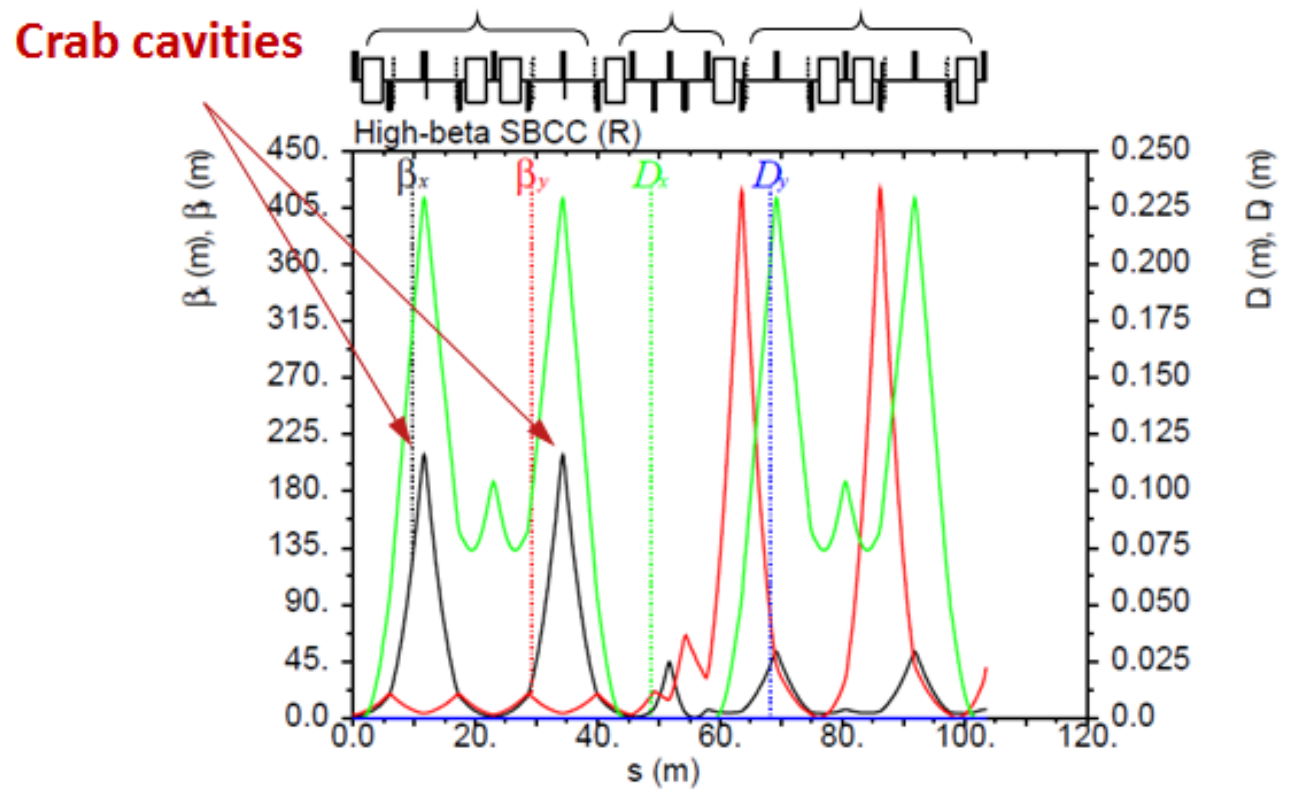

FIG. 30: Optics of the Chromatic Compensation Blocks of the electron ring [6].

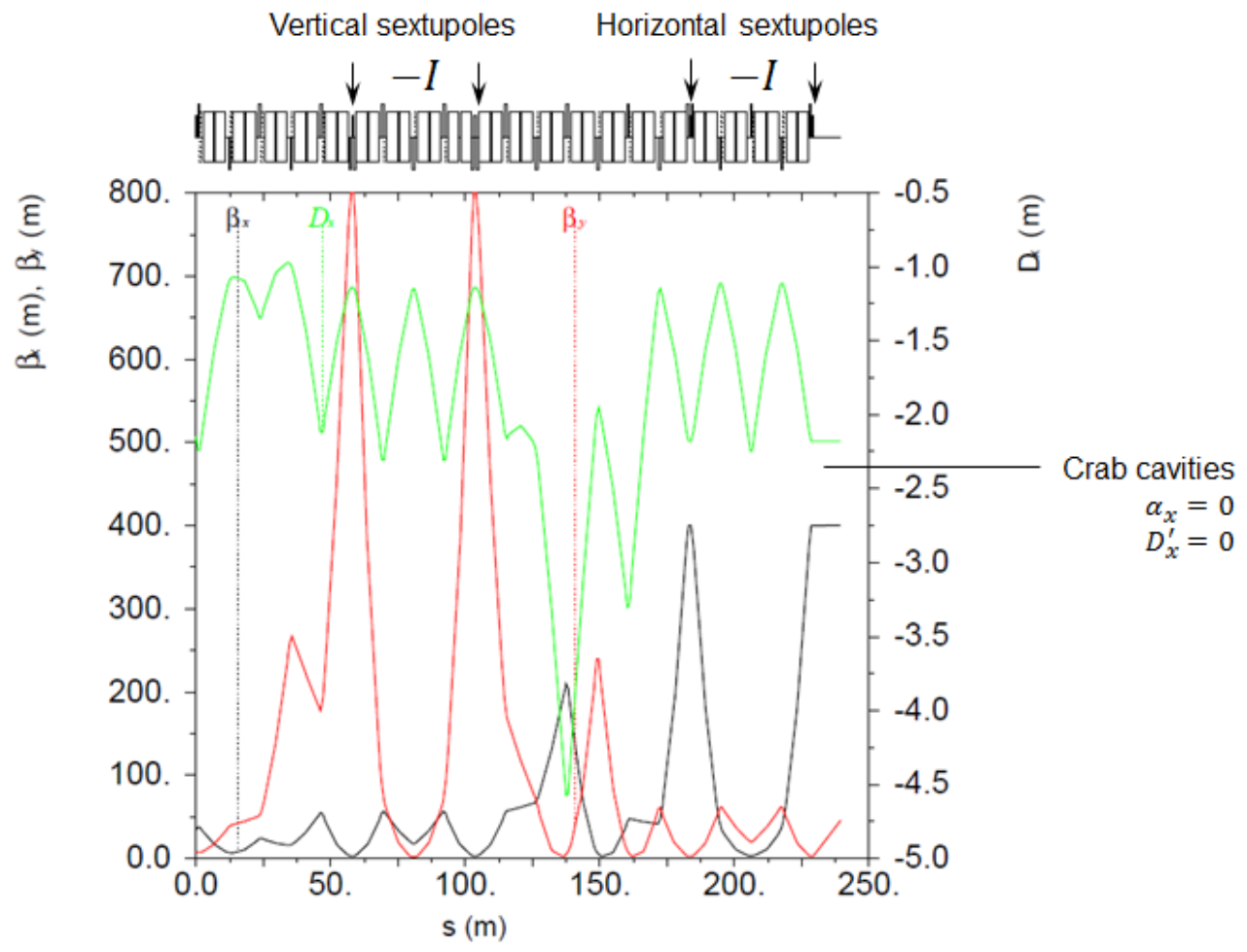

FIG. 31: Optical sections of the CCB for the ion ring. This includes $10 \mathrm{~m}$ space reserved for crab cavities at each side of the interaction region [6]. 
the IP is [39]

$$
V=\frac{c E_{b}}{e \omega \sqrt{\beta_{\text {crab }} \beta^{*}} \sin \left(\psi_{C C \rightarrow I P}\right)} \cdot \tan \left(\frac{\phi_{\text {cross }}}{2}\right)
$$

where $E_{b}$ is the beam energy, $\phi_{\text {crab }}$ is half the crossing angle, $\beta_{\text {crab }}$ is the horizontal beta function at the crab cavity location and $\beta^{*}$ is the horizontal beta function at the IP. General crabbing parameters for JLEIC are presented in Table 5 .

TABLE 5: Bunch crabbing parameters of interest for both electron and proton beams in JLEIC.

\begin{tabular}{|l|c|c|c|c|c|c|}
\hline Parameter & Electron ring & \multicolumn{3}{|c|}{ Ion ring } & Units \\
\hline Energy & 5 & 10 & 20 & 60 & 100 & $\mathrm{GeV}$ \\
\hline Frequency & \multicolumn{5}{|c|}{952.6} & $\mathrm{MHz}$ \\
\hline Crossing angle & \multicolumn{5}{|c|}{50} & $\mathrm{mrad}$ \\
\hline$\beta_{x}^{*}$ & \multicolumn{5}{|c|}{0.1} & $\mathrm{~m}$ \\
\hline$\beta_{x}^{\text {crab }}$ & \multicolumn{2}{|c|}{200} & \multicolumn{3}{|c|}{363.44} & $\mathrm{~m}$ \\
\hline$\eta_{x}^{\text {crab }}(\mathrm{crab} 1 / \mathrm{crab} 2)$ & \multicolumn{2}{|c|}{$0.25 /-0.25$} & \multicolumn{3}{|c|}{$-2.15 / 2.15$} & $\mathrm{~m}$ \\
\hline$\Delta \psi_{\mathrm{CC} \rightarrow \mathrm{IP}}($ crab 1/crab 2) & $7.52 \pi / 10.52 \pi$ & \multicolumn{3}{|c|}{$4.5 \pi / 9.5 \pi$} & - \\
\hline Crab voltage & 1.4 & 2.8 & 4.18 & 12.50 & 20.82 & $\mathrm{MV}$ \\
\hline
\end{tabular}

At the CCB locations where the crab cavities are placed, the tolerance criterion on the dispersion of the ring given in Eq. (106) is well satisfied: $\left|\eta \eta^{\prime}\right|=0.4565 \mathrm{~m}<5.0763 \mathrm{~m}$.

\subsubsection{CRAB CAVITY APERTURE}

The cavity aperture corresponds to the cavity smallest transverse size, and it determines the minimum space allowance for the beam to pass. The aperture must be compatible with the beam during beam injection, when the beam has the largest size [44]. Figure 32 shows the injection optics for the ion ring.

At injection, the normalized beam emittance is $\epsilon_{x}=1 \mu \mathrm{m}$, and the momentum spread $\Delta p / p=1 \times 10^{-3}$. At the CCB locations, the horizontal beta function and dispersion values are: $\beta_{x}=37.5 \mathrm{~m}$ and $\eta_{x}=2.2 \mathrm{~m}$. The beam size can then be calculated using Eq. [70), which gives $\sigma_{x} \simeq 3.1 \mathrm{~mm}$ at the location of crab cavities. A cavity aperture of $10 \times \sigma_{\max }$ does not leave space for closed orbit excursion of the beam. A conservative aperture value of 70 $\mathrm{mm}$ is recommended, which gives room for further optimization on the optics near the crab cavity sections. Careful orbit control and beam collimation in this region will be required [45]. 


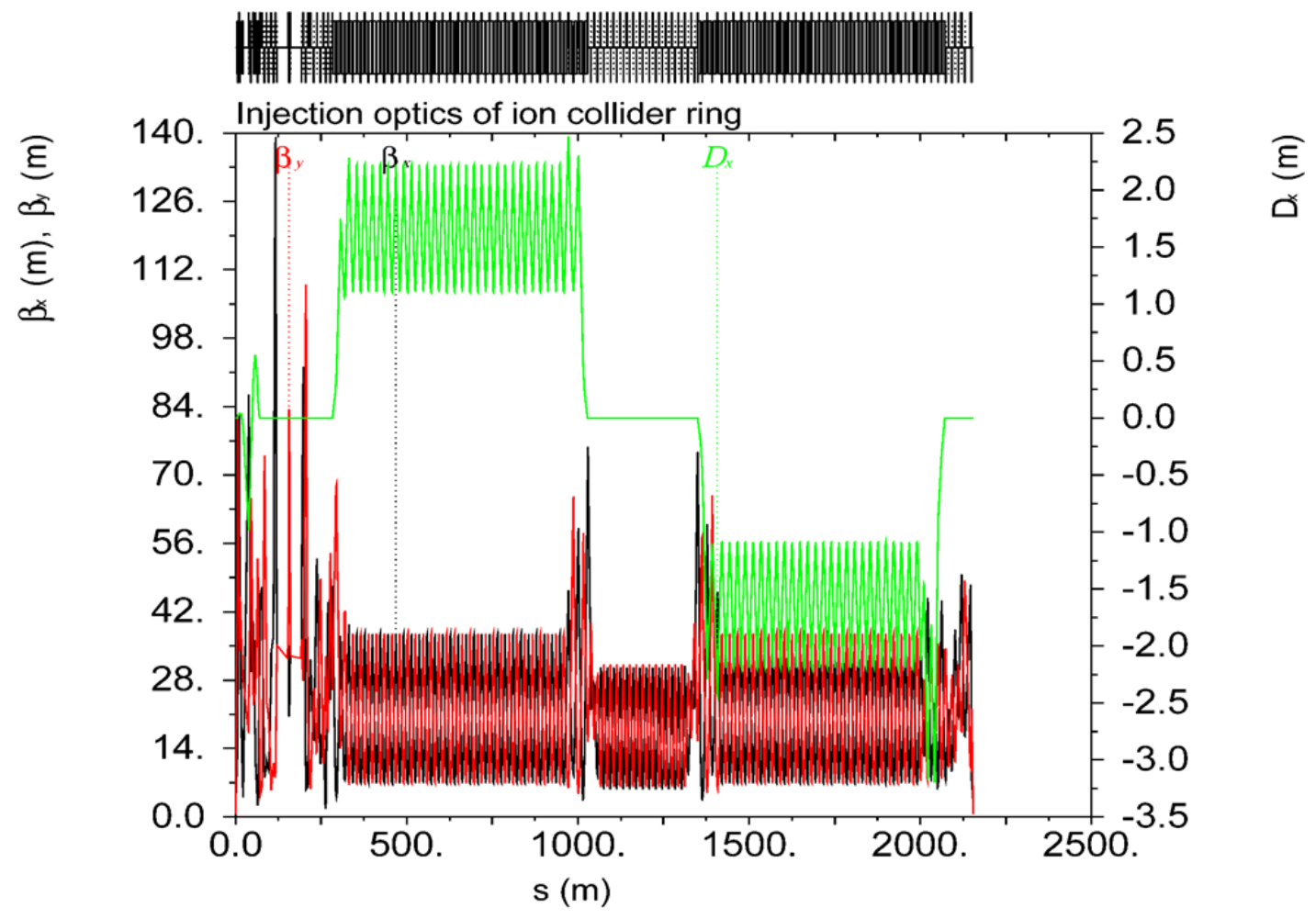

FIG. 32: Injection optics of the ion ring. 


\section{CHAPTER 5}

\section{SIMULATIONS}

As it was described at the beginning of the previous chapter, the design of a particle accelerator is an iterative process, where one starts with an ideal beam trajectory that may evolve multiple times into the final machine. Accelerators are in general constructed from well-understood components, namely dipole or quadrupole magnets, rf cavities, collimators, etc. It is possible to model all these elements in a computer to make an accurate representation of the particle accelerator, and then make a beam of particles travel through it in order to get an insight into the dynamics of the beam in the machine. There are currently many different accelerator codes, each suitable for specific applications and may include numerous physics environments happening in a real accelerator.

The results from the SPS test with crab cavities are very important and may give important information for the design of crabbing regions for EIC. At the time of writing this dissertation, however, dedicated beam runs for crabbing of proton beams at SPS have just started. For the purposes of present JLEIC studies, we rely on particle tracking studies as a starting point into the dynamics of JLEIC beams when the rings include crab cavities. Privous beam tracking simulations using rf deflecting cavities for CEBAF [46], and crab cavities for MEIC have been performed [47]. In the MEIC simulations, a transfer matrix was used to propagate the beam through the MEIC ring. In this dissertation, we use full description lattices of the most recent version of the rings and track individual particles at every location.

This chapter is arranged in the following way: Section 5.1 discusses the tracking of particles using elegant and describes the JLEIC rings and crab cavity models used. In Section 5.2 , we introduce bunch crabbing elements into the JLEIC rings to simulate a local crabbing scheme that is consistent with the considerations in Section 4.4. In Section 5.3, we study the effect of cavity multipoles on the beam dynamics of JLEIC, in which the multipoles correspond to actual crab cavity designs modeled in CST Microwave Studio. Finally, in Section 5.4, we study the effect of crab voltage noise in the stability of JLEIC beams.

\subsection{PARTICLE TRACKING}


The present work relies principally on two accelerator codes: MAD-X [48] and elegant [49]. MAD-X (Methodical Accelerator Design) is code developed at CERN and widely used for optical design of a particle accelerator. Elegant was developed at Argonne National Laboratory (AND) and is a code used for particle tracking studies. The lattices corresponding to JLEIC rings used in this dissertation were created using MAD-X by Vasiliy Morozov and Fanglei Lin. They were then translated into elegant format using a Python script. These ring lattice files are presented in Appendix B.

\subsubsection{ELEGANT}

The name elegant stands for ELEctron Generation ANd Tracking, although its capabilities have been extended since its original release [49]. Elegant tracks the 6D phase space coordinates $\left(x, y, x^{\prime}, y^{\prime}, s, \delta\right)$ of individual particles in the beam after each of the accelerator components defined by the user. Here, $x$ and $y$ are the horizontal and vertical displacements of a particle with respect to the ideal beam path, $x^{\prime}=d x / d s$ and $y^{\prime}=d y / d s$ are the slopes, $s$ is the total distance traveled by the particle and $\delta=\Delta p / p_{0}$ is the fractional momentum deviation. Elegant allows one to define both the particle beam and the lattice array that makes up the machine. Accelerator elements can be represented either by matrices or by canonical elements up to a certain order.

Elegant uses Self Describing Data Sets (SDDS), which is a versatile file protocol with a variety of capabilities. Together with the SDDS toolkit, which was also developed and supported at ANL, the processing of elegant output becomes very flexible, as it is possible to easily manipulate data within the output file itself, from plotting output files, to performing statistical analysis or Fast Fourier Transforms. It also includes tools to convert elegant output file format into other accelerator codes, like Astra, or regular text files. It is very easy to implement routines using Python and automate processes involving elegant.

\subsubsection{CRAB CAVITY MODEL}

A crab cavity is modeled in elegant as a thin element that changes the transverse momentum of particles in a bunch, where the added transverse momentum depends on the particle location relative to the bunch center. In elegant, this can be represented by an RF DeFlector (RFDF) thin element, where the changes in the momentum components, in units 
of $m c$, gained by a particle after passing through the cavity element are

$$
\begin{aligned}
& \Delta p_{x}=\frac{q V}{m c^{2}} \cos \omega t, \\
& \Delta p_{y}=0, \\
& \Delta p_{z}=\frac{q V}{m c^{2}} k x \sin \omega t,
\end{aligned}
$$

with $V$ the crabbing voltage. The deflection of a particle in the bunch is given by $\Delta x_{k}^{\prime}=$ $\Delta p_{k} / p_{z}$. The term $\Delta p_{z}$ follows from the Panofsy-Wenzel theorem [24] presented in Section 3.3. The crab cavity is modeled as a thin element with length $\Delta L=0$. For the time being, none of the JLEIC rings available for crabbing simulations has reserved space for the crab cavities. Nevertheless, the thin cavity approximation should be acceptable as long as the betatron wavelength does not change significantly near the cavity locations, i.e., long betatron wavelength. A future study of the crabbing dynamics with updated rings would include a finite length cavity. By having a dedicated space for the cavity, a field map can be used, enabling more realistic cavity modeling.

Another assumption in modeling the crab cavities is that a single crab cavity element provides the full required voltage. In reality, however, the voltage that an individual crab cavity can provide is of course limited by the peak surface fields in the cavity. For a multi-cell rf-dipole crab cavity type [32], the number of cavities needed is between 10 and 12 per side of the interaction point for the ion beam, for a total of 20 to 24 cavities in the ion ring. In the case of the electron beam, only about 2 cavities per side of the interaction point are needed. These numbers take into consideration the cavities required to produce the tilt plus spare cavities to ensure luminosity production in the case a cavity trips.

We now describe the different models of the JLEIC rings used in the particle tracking simulations of Section 5.2 .

\subsubsection{ION RING}

We consider two different versions of the ion ring: the first one has the vertical CCB closer to the interaction point. In the second one, the horizontal CCB are closer to the interaction point. Both ring configurations are described in this section.

\section{Ion ring lattice with switched hor./vert. CCB}

At the beginning of this project, the JLEIC ion ring lattice available for simulations was the one depicted in Fig. 33. This ion ring is an 8-shaped ring with a circumference of 2183.59 
$\mathrm{m}^{1}$. It is formed by two $261.7^{\circ}$ arcs and two connecting straight sections that cross each other forming an $81.7^{\circ}$ angle. One of the straight sections hosts the interaction region, while the other one hosts the SRF systems, as well as space for a second interaction region in a future upgrade. The collision optics of this ring is also presented in Fig. 34, which shows the horizontal and vertical betatron functions, as well as the dispersion function at locations along of the ring. Through the rest of the dissertation, we refer to this lattice as either the old version of the ion ring, or lattice 1 ion ring. We reserve the name baseline ion ring for the lattice described at the end of Section 5.1.3.

To be more specific, the lattice in Fig. 34 corresponds to the collision optics of the ion ring, which is where bunch crabbing is required. Traveling along the lattice, the position at $s=0 \mathrm{~m}$ corresponds to a position at the center of a quadrupole located in the middle of the straight section opposing the interaction region. From $s=0 \mathrm{~m}$ to about $s=100 \mathrm{~m}$ there is a part of the straight section. Then comes one of the arc sections from about $s=100 \mathrm{~m}$ to $s=500 \mathrm{~m}$. The interaction region spans from $s=500 \mathrm{~m}$ to about $s=1500 \mathrm{~m}$, with the interaction point being near the $s=1000 \mathrm{~m}$ mark. The second arc section spans from about $s=1500 \mathrm{~m}$ to $s=2000 \mathrm{~m}$, coming back to the initial straight section and closing the ring lattice.

The $s=0 \mathrm{~m}$ location is not a particularly special location of the ring, but it is rather chosen as a starting point for the crabbing simulations for practical reasons. First, because it is a point outside the crabbing region, i.e., this allows us to define a particle distribution outside the crabbing region and then study the effects of the crabbing system. Second, $s=0$ $\mathrm{m}$ corresponds to a point at the center of a quadrupole, where the Twiss parameter $\alpha$ is zero. This is convenient because in elegant, a distribution at this point corresponds to an upright ellipse, which is straightforward to define.

Important features to recognize in Fig. 34 are the locations of the CCBs, where the chromatic sextupoles are located. These locations correspond to the pairs of high beta function values, or peaks. There is a pair of horizontal beta peaks before and after the IP, and similarly, a pair of vertical beta peaks before and after the IP. Furthermore, it should be noted that in this ring, the vertical CCB are closer to the IP than the horizontal CCB. The betatron phase advances at the locations of horizontal chromatic sextupoles are $\Delta \psi_{1}=7.5 \pi$ from the first CCB to IP and $\Delta \psi_{2}=12.5 \pi$ from the IP to the second horizontal CCB as pointed in Fig. 34 with blue arrows. These phase advances are consistent with the required phase advance condition, Eq. (104), in the local crabbing scheme.

\footnotetext{
${ }^{1}$ As can be noted, this ring is a bit shorter than the newer version of the ring presented in Chapter 2
} 


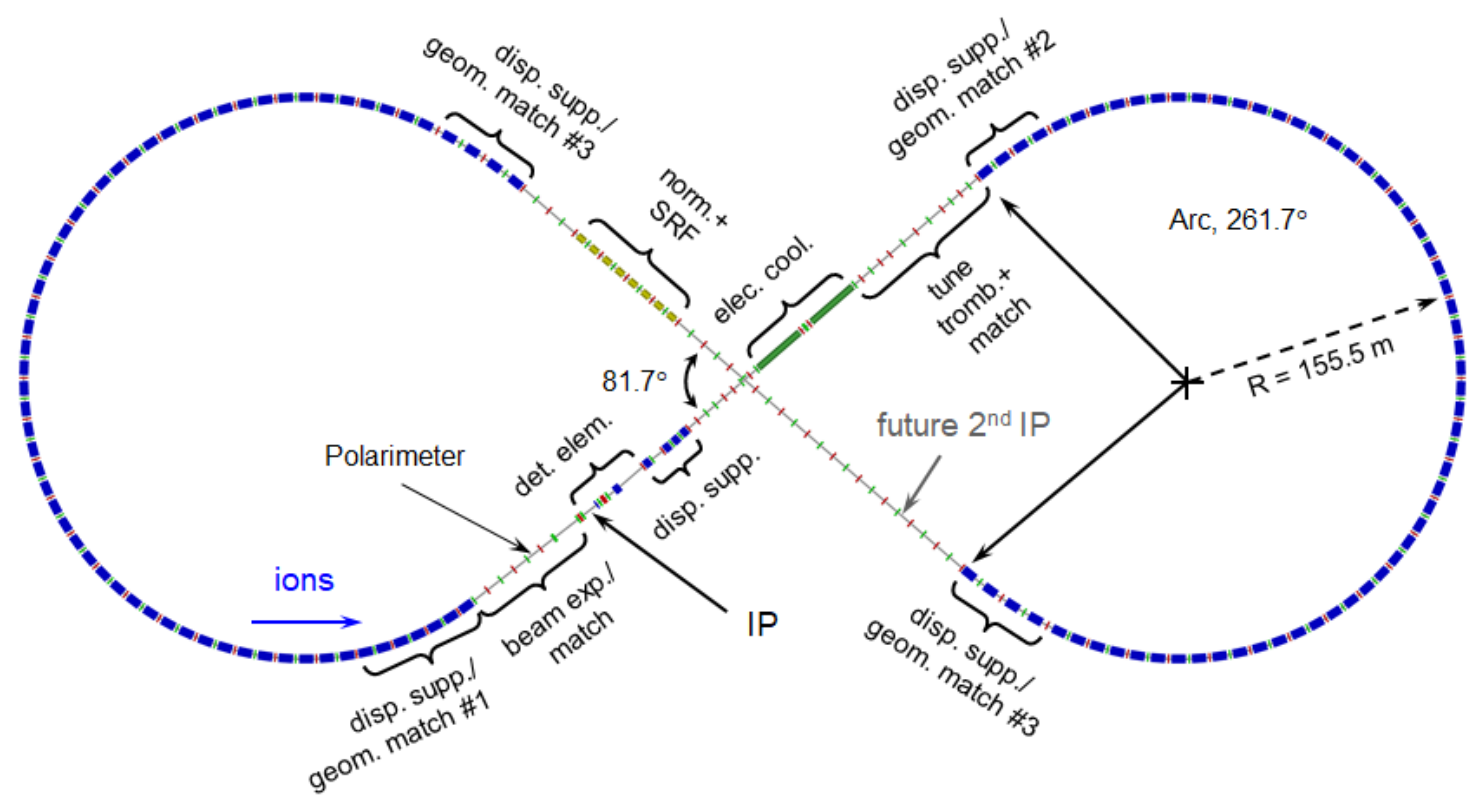

FIG. 33: First version of the JLEIC ion ring where local crabbing was implemented, the circumference of the ring is $2153.9 \mathrm{~m}$.

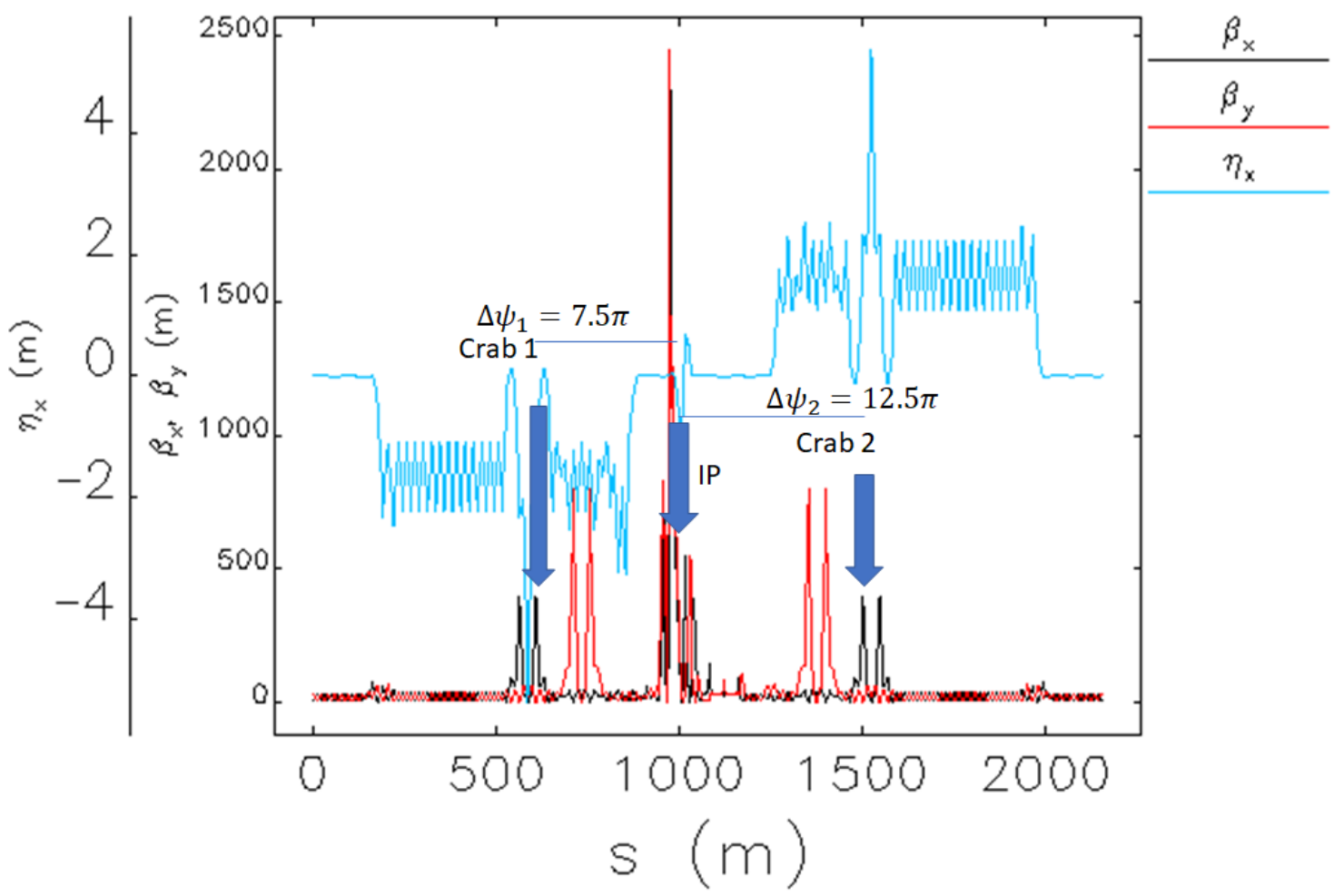

FIG. 34: Optics of the ion ring lattice with the vertical CCB closer to IP. 
A local crabbing scheme consistent with the parameters in Table 5 was first implemented in this lattice for a $100 \mathrm{GeV}$ proton beam. Since the crab cavities are placed at the locations of the two horizontal chromatic sextupoles that are closest to the IP, the bunches are already crabbed when they travel across the vertical CCBs. As will be discussed in Section 5.2 , this configuration introduces non-linear effects from the vertical CCS in the crabbed bunch, which increases the beam emittance significantly. In order to avoid this, a new version of the ion ring with different CCB arrangement was developed.

\section{Ion ring baseline lattice}

Figure 35 shows a modified collision optics of the old ion ring, where the vertical and horizontal CCB locations have been switched. By switching the vertical CCS outside of the

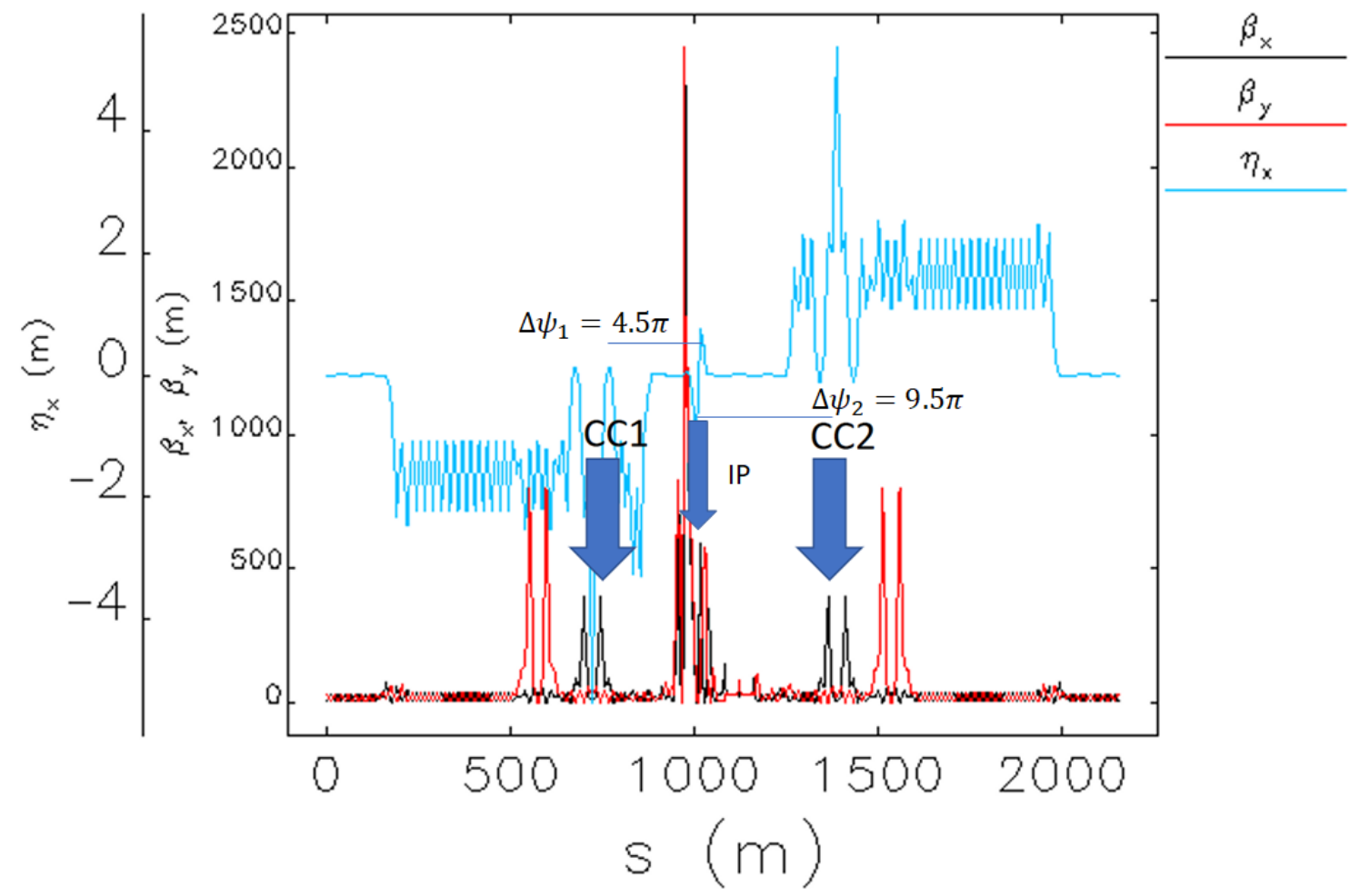

FIG. 35: Collision optics of the JLEIC lattice with switched horizontal/vertical CCB.

region where the bunches are crabbed, the non-linear effects of the vertical CCS are now avoided. This change however, comes at a price. In this new configuration, the horizontal beta functions at the $\mathrm{CCB}$ are reduced to $\beta_{x}=363.44 \mathrm{~m}$, which in turn increases the voltage requirement of the crabbing system. Adjusted voltage values for this lattice are presented in Table 6 for different ion beam energies. 
TABLE 6: Adjusted crab cavity voltage due to a lower $\beta_{\mathrm{crab}}^{x}$ in the baseline ion ring.

\begin{tabular}{|c|c|}
\hline Energy $[\mathrm{GeV}]$ & Crab Voltage $[\mathrm{MV}]$ \\
\hline 20 & 4.18 \\
\hline 60 & 12.50 \\
\hline 100 & 20.82 \\
\hline
\end{tabular}

Similarly, the betatron phase advances of the horizontal CCB with respect to the IP are now $\Delta \psi_{1}=4.5 \pi$ for the upstream CCB and $\Delta \psi_{2}=9.5 \pi$ for the downstream CCB. These phase advances are also in agreement with a local crabbing scheme.

Most of the results presented in further sections of the present Chapter correspond to crabbing simulations using this version of the ion ring lattice.

\subsubsection{ELECTRON RING}

The optics of the available electron ring is shown in Fig. 36. This ring is still being developed and has not yet been optimized for crabbing; thus only preliminary results are presented. It is expected, however, that once a stable version of this ring becomes available, the analysis presented for the ion ring would translate almost directly into the electron ring. In this lattice, the horizontal CCB are closer to the IP than the vertical ones, which avoids non-linear coupling from the vertical sextupoles and the crabbed bunch. The beta functions at this CCB peaks are $\beta_{x}=200 \mathrm{~m}$. Even though this value is lower than the beta functions in the ion ring, the parameters for the crabbing of electrons are less stringent than for ions. For example, the required crabbing voltage is only a few $\mathrm{MV} / \mathrm{m}$, which can easily be achieved with just a couple of crab cavities per side of the IP. In the electron ring, the starting point is selected at the opposing straight section to the interaction region for the exact same arguments as in the ion ring. The arc sections are also formed by FODO lattices.

We now proceed to describe the implementation of a bunch crabbing system in the three lattices discussed in this section.

\subsection{JLEIC MODEL WITH BUNCH CRABBING}

Elegant can record the phase space of particles through WATCH elements. These can be placed anywhere in the ring without altering the dynamics in any way. We can place a WATCH element at the beginning of the ring to record the initial particle distribution in the ion ring for a $100 \mathrm{GeV}$ proton bunch, shown in Fig. 37. This bunch corresponds to a 


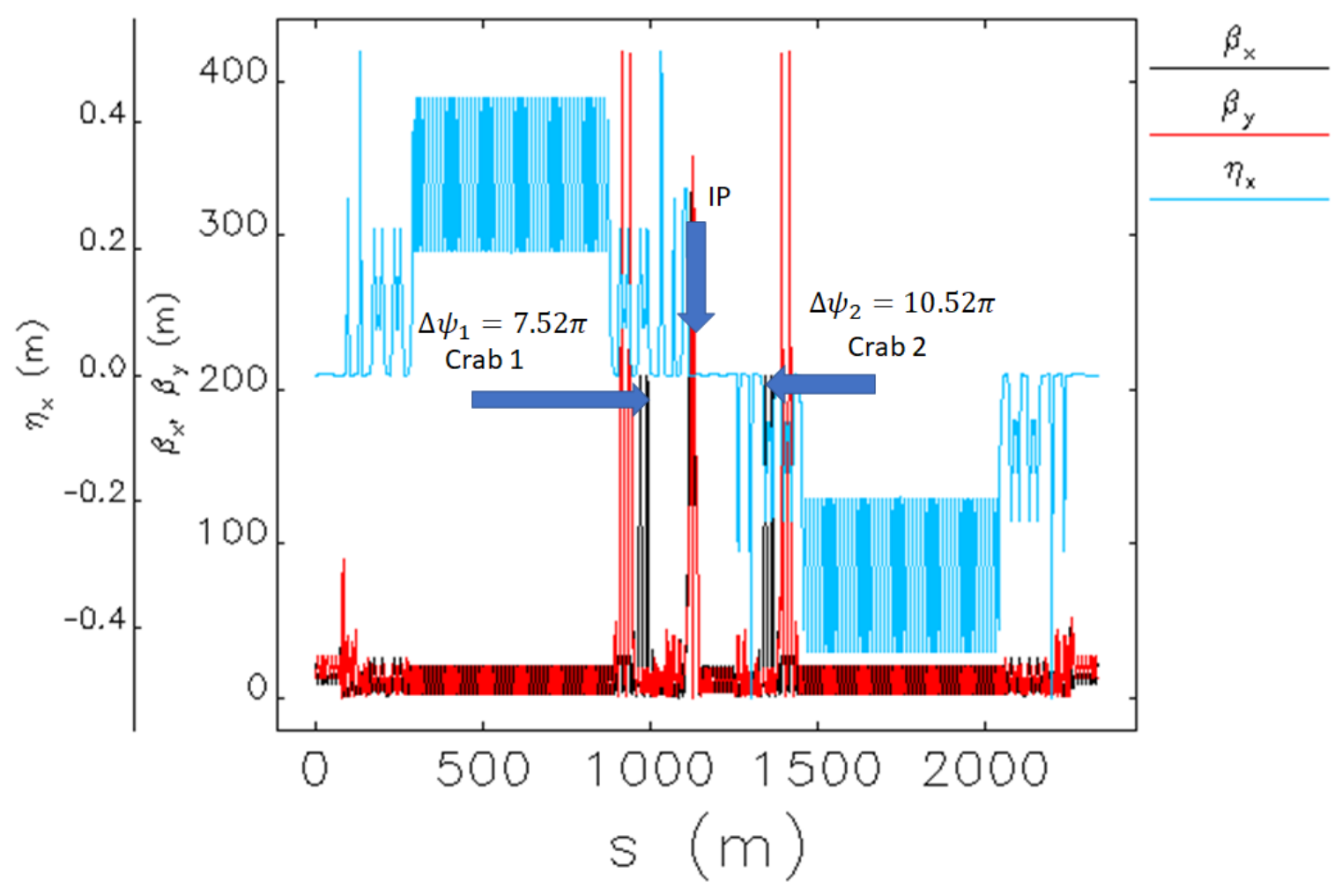

FIG. 36: Electron ring collision lattice.

Gaussian distribution of 10000 particles generated at $s=0 \mathrm{~m}$.

The bunch length is taken to be the rms standard deviation of the distribution and $\sigma_{z}=1 \mathrm{~cm}$. Each particle is tracked individually along the ring for exactly 1 turn. The normalized horizontal and vertical emittance of the ion beam are $\epsilon_{x}=0.35$ and $\epsilon_{y}=0.07$ $\mu \mathrm{m} \cdot \mathrm{rad}$, which correspond to an ion beam with strong cooling.

We first implement crab cavity elements as described in Section 5.1.2. A first step is to verify that we are, in fact, producing the required tilt at the interaction point. Figure 38 shows a snapshot of crabbed electron and ion bunches at the interaction point. We include a bunch produced when the crab cavities are turned off (0 MV crabbing voltage) to compare the bunch tilt at IP due to the crab cavities.

Note in Fig. 38 that even though the bunches shown correspond to both electrons and protons at different energies, the produced tilt is still the required $25 \mathrm{mrad}$ angle for each of them. The relationship between crabbing voltage and beam energy comes from Eq. (109). 


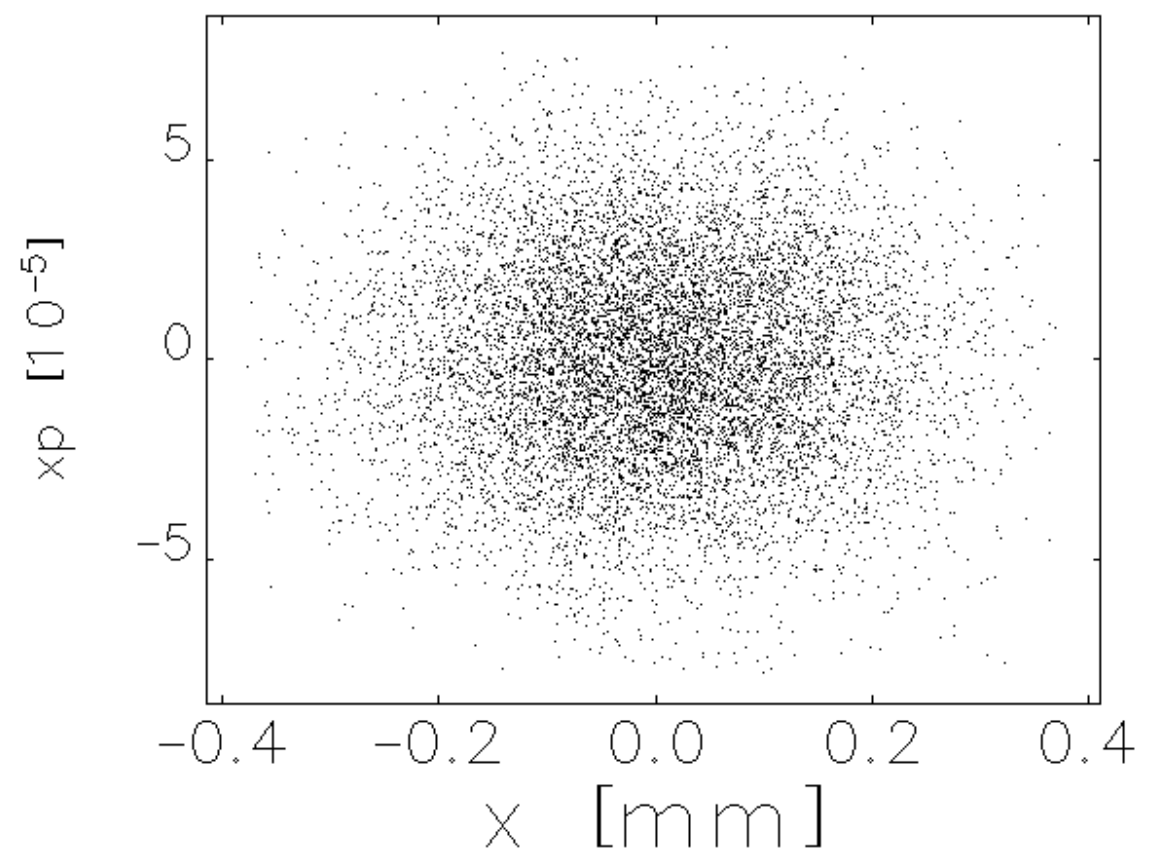

FIG. 37: Initial distribution of a $100 \mathrm{GeV}$ protons bunch with 10000 particles.

We next use smaller Gaussian bunches of 1000 particles assuming the same parameters and track them over many turns in order to evaluate the stability of beams when the crabbing system is turned on.

Figure 39 corresponds to the lattice 1 ion ring set up for a $100 \mathrm{GeV}$ proton beam and shows the calculated bunch angle at the interaction point as a function of the number of turns. The crab angle $\phi_{\text {crab }}$ is calculated at each turn through the statistical average of the transverse and longitudinal correlations,

$$
\phi_{\text {crab }}=\frac{\langle x z\rangle}{\left\langle z^{2}\right\rangle} .
$$

given the coordinates of each particle in the beam.

In the following, all the rings considered include crab cavities and the convention on the labels is as follows: in "Crab On" situations, the crabbing voltage is set up accordingly, whereas for "Crab Off" scenarios, the RFDF element is present in the ring, but its voltage is kept at $0 \mathrm{~V}$, i.e., no effect on the beam. It is possible to see in Fig. 39 that the angle is zero when the crab cavity voltage is set to zero and close to $25 \mathrm{mrad}$ when the crab cavity voltage is on. It is noticeable however, that the crab angle exhibits some level of variation over time. 


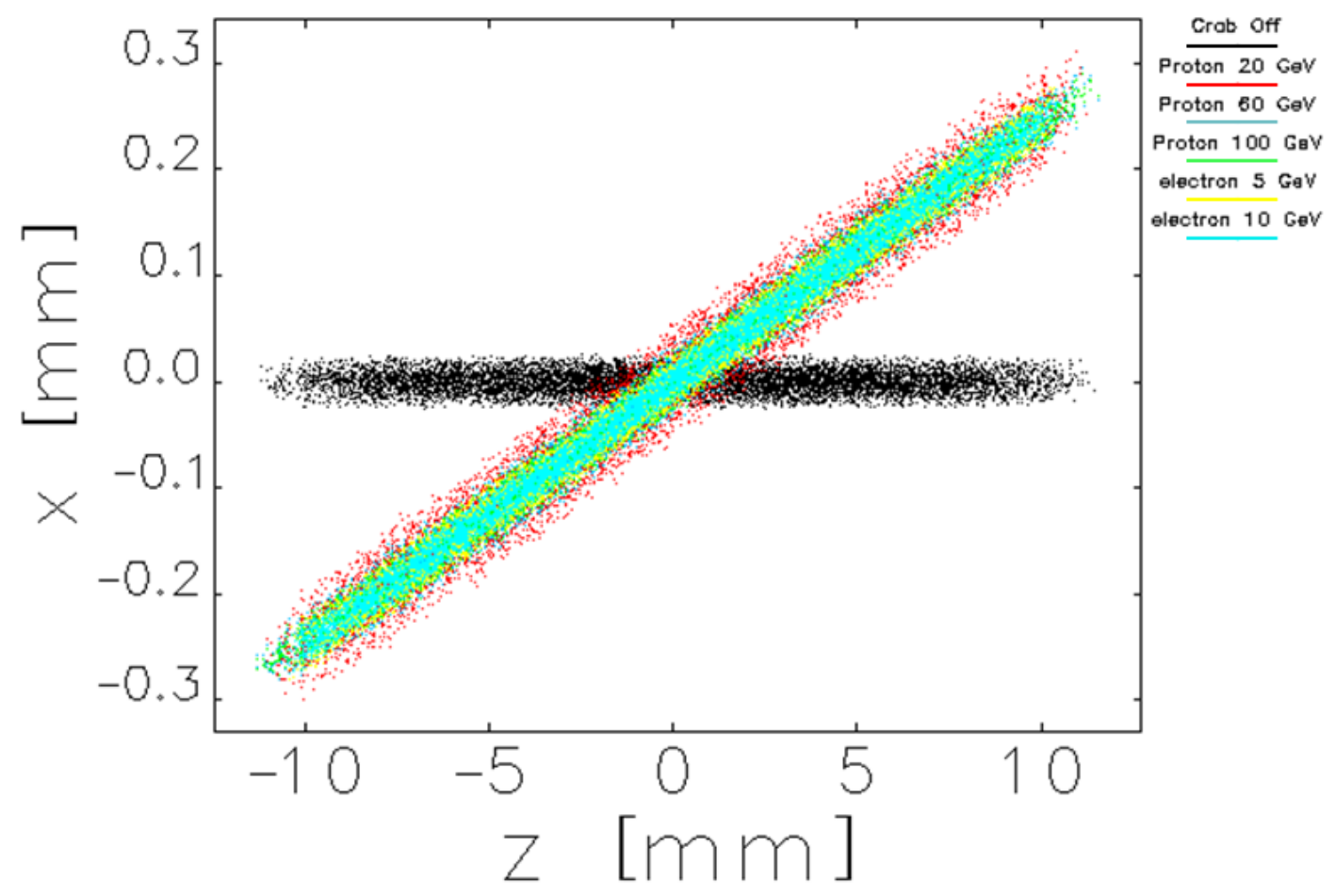

FIG. 38: Crabbed electron and ion bunches at IP at different energies.

Furthermore, Fig. 40 shows the corresponding time evolution of the normalized horizontal emittance in this lattice and we can see that it grows steadily and eventually blows up.

As it was previously mentioned, in this ion ring lattice, the vertical chromatic sextupoles (CCS) are located inside the region of the ring where the bunches are crabbed. Simulation suggests a strong destructive coupling of the vertical CCS non-linear fields with the crabbed bunch that increases the emittance and eventually destroys the beam.

In order to avoid coupling to the vertical CCS observed in the lattice 1, a modified version of the ion ring was implemented, where basically the horizontal and vertical CCB are swapped places to leave the vertical CCS out of the crabbing region. With this new configuration, Fig. 41 shows again the evolution of the crabbed bunch angle at the IP as a function of the number of turns, and it can be noted that the angle is pretty stable as compared to the the lattice 1 case, even at earlier turns.

In this case, the crabbing angle is shown for the ion beam at different energies. The corresponding beam emittance is found to grow in a small, correlated behavior, as will be 


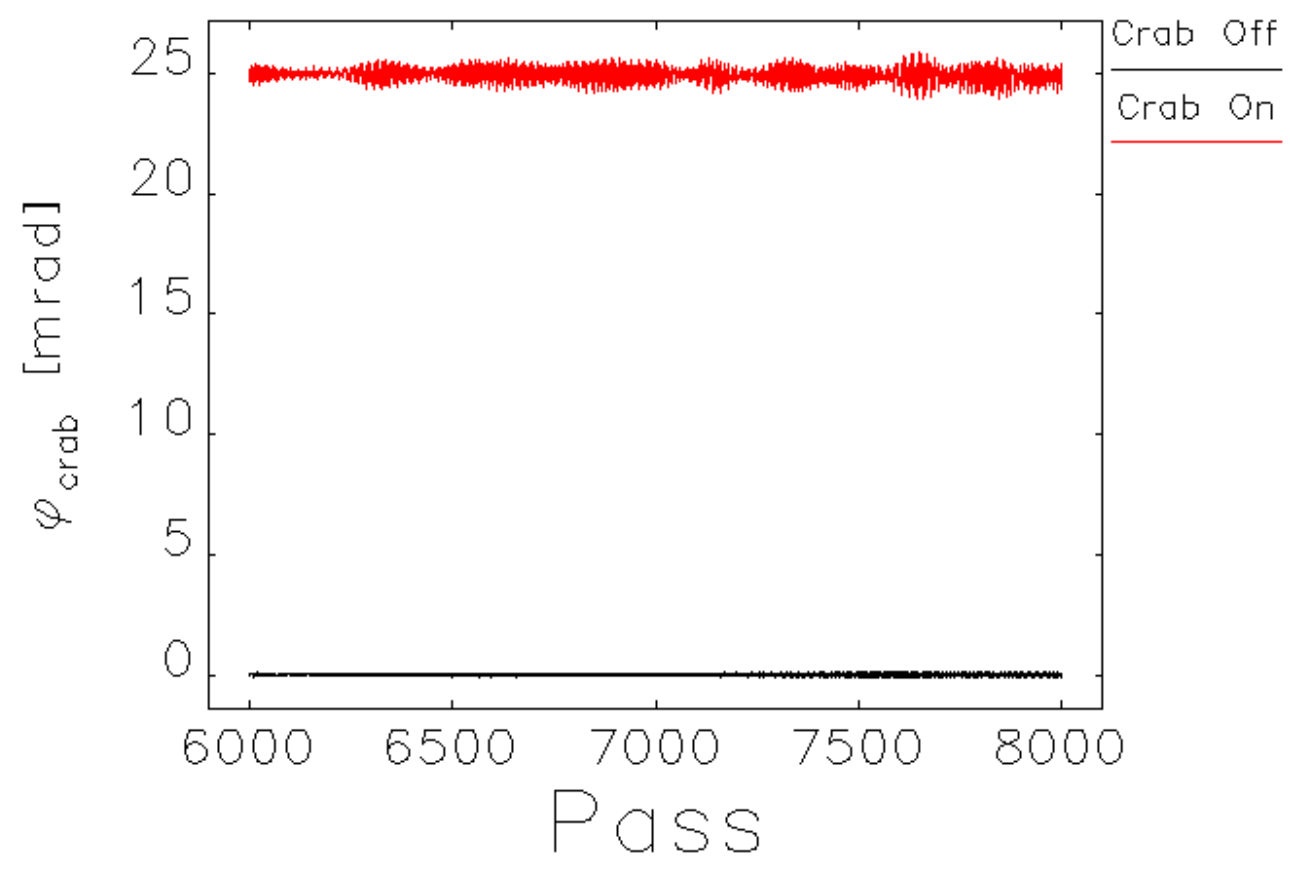

FIG. 39: Crab angle at the IP in the baseline ion ring lattice, Lattice 1.

explained in Section 5.2.1. Also, because this is the more stable ring when the effect of bunch crabbing is included, we use it for the studies of cavity multipoles and rf noise in Sections 5.3 and 5.4

In a similar way, we implement crabbing elements in the available version of the JLEIC electron ring. First let us note back in Fig. 36 that in this lattice the relative phase advances from the corresponding crab cavity locations to the IP are not exactly set up to the phase advance condition in Eq. (104). Once the electron ring becomes more developed, it can be further optimized for crab cavity dynamics. Nevertheless, we study as well the effects of a linear bunch crabbing in this lattice.

Figure 42 shows the crab angle evolution in the electron ring using a $5 \mathrm{GeV}$ electron beam. It is clearly visible that there is some sort of oscillating behavior on the angle formed at the interaction point, even when the crabbing system voltage is zero. In Fig. 43, we show the bunch tilt at the location of the first crab cavity. This suggest that the crab cavity effect on this beam is to amplify an already present oscillation in the ring. We investigate the frequency content of this oscillations by tracking the bunch centroid position at every turn and, through a Fast Fourier Transform implemented with elegant, we can get the fractional 


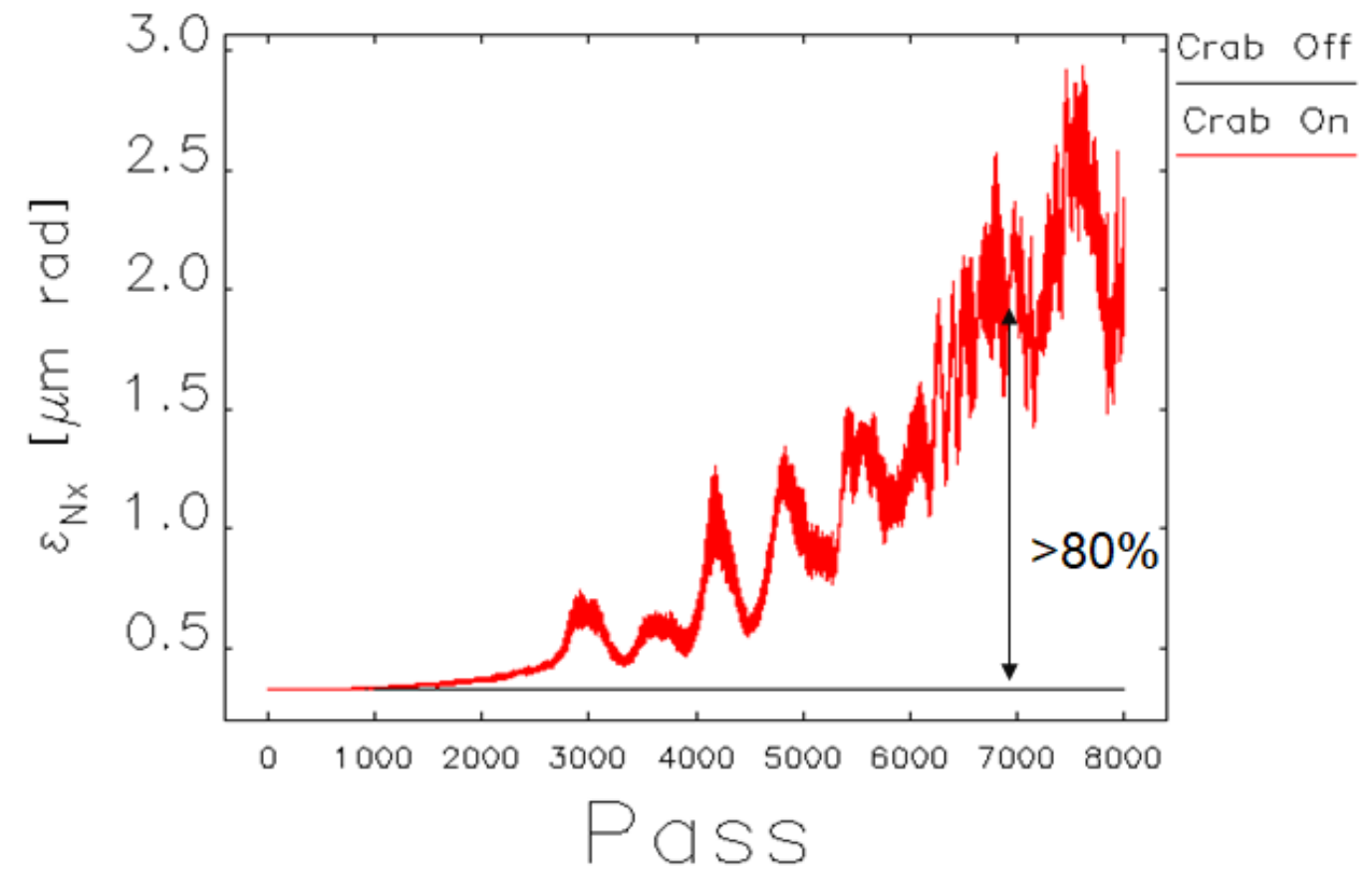

FIG. 40: Emittance growth due to crab cavities in the baseline ion ring lattice.

tunes of the ring, which are shown in Fig. 44 .

The fractional tunes for the electron ring are $\nu_{x}=0.22, \nu_{y}=0.16$ and $\nu_{z}=0.018$. Note that the peaks shown correspond to locations at $\nu_{x} \pm \nu_{z}$ and $\nu_{y} \pm \nu_{z}$, respectively. This suggest that there is synchro-betatron coupling of the beam, that gets amplified by the kick introduced by the crab cavities.

\subsubsection{EFFECT OF CAVITY VOLTAGE TURN ON RATE}

In order to study the emittance increase due to the crab cavities in the baseline lattice, we slowly ramp the voltage over many turns rather than turning it on instantaneously. In Fig. 46, we present the effect on the beam emittance of different crab cavity voltage turn on times: 10, 100, 500, 1000 and 2000 turns for comparison, where each turn along the ring for $100 \mathrm{GeV}$ proton beam is about $0.7 \mu \mathrm{s}$. The beam is left crabbed for some time before being turned off over 2000 turns to allow for comparison. This emittance increase seems to be a correlated effect [39, 50]. The crabbing kick may not be exactly compensated, causing non-zero correlations between the horizontal and longitudinal degrees of freedom. 


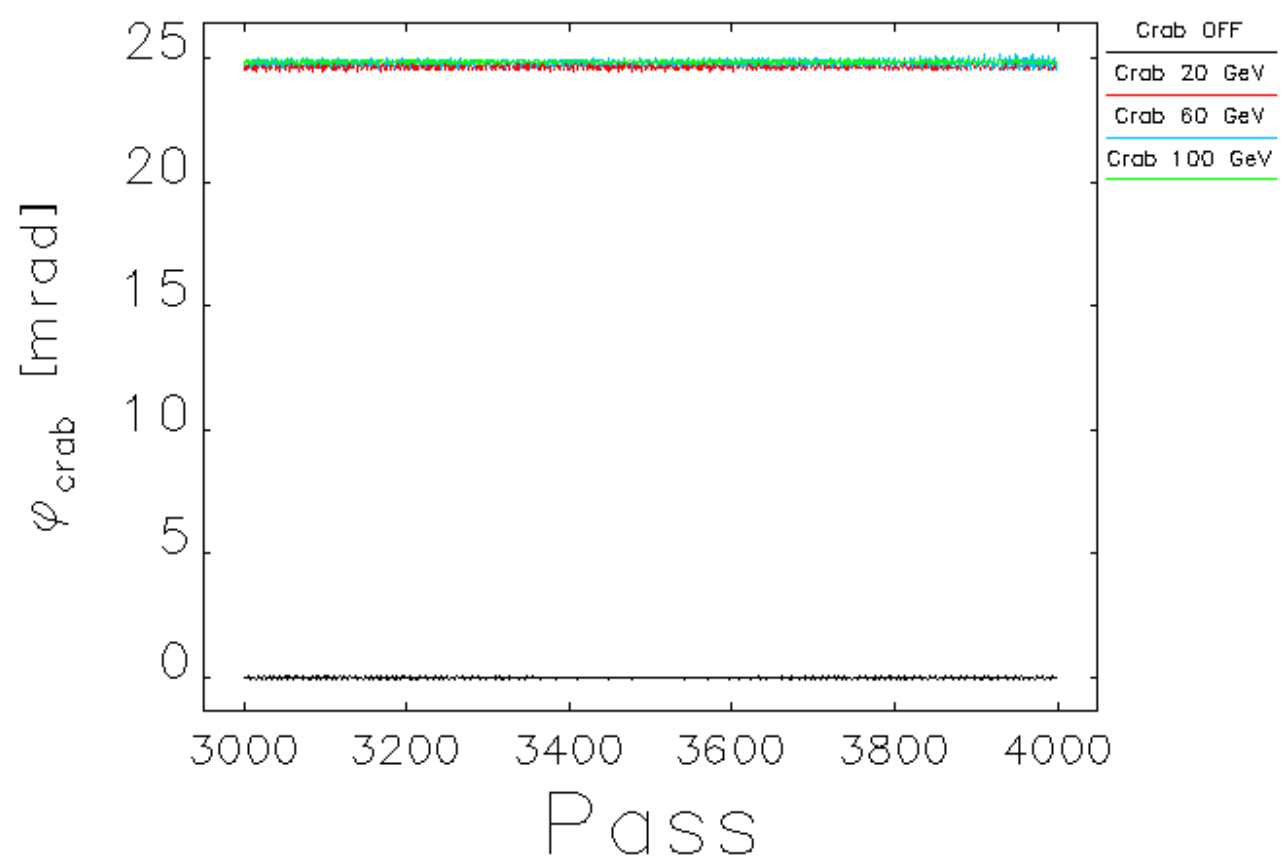

FIG. 41: Crab angle evolution at the IP in the lattice with switched CCB. Angle is shown for low, medium and high energy proton beam.

This establishes a new matched 6D phase-space ellipsoid around the ring. Note that when the crab voltage is ramped over 10 and 100 turns, there exists a residual emittance increase after the voltage is turned off. Otherwise, the non-correlated phase-space emittance is recovered as long as the crab cavity voltage turn-on is done adiabatically over at least 500 turns. This corresponds to a minimum turn-on time of a few hundred turns (milliseconds) for minimum emittance impact. This time is in agreement with the loading time of a typical $Q \sim 10^{6}$ cavity, and thus represents a consistent simulation for JLEIC. 


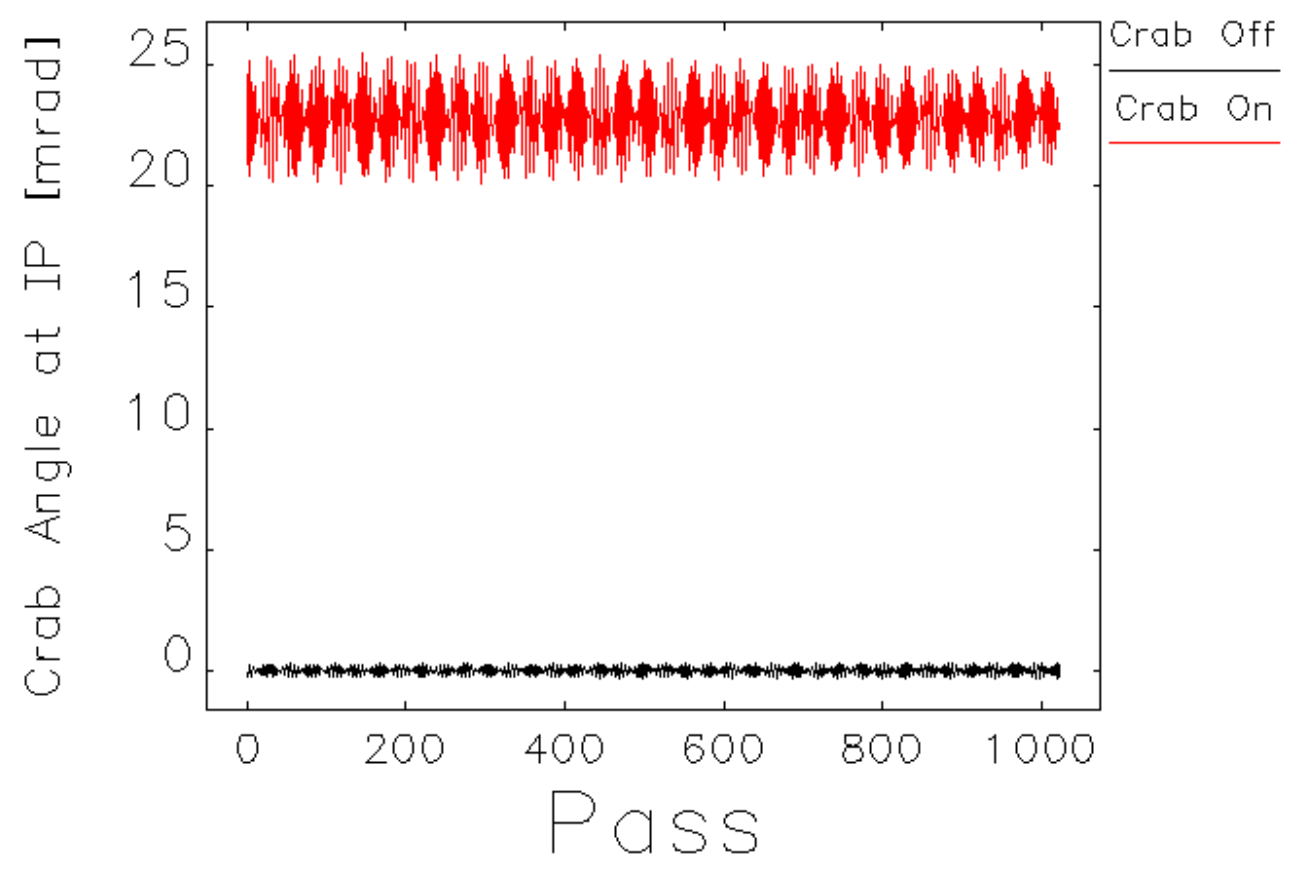

FIG. 42: Crab angle evolution in the electron ring at $5 \mathrm{GeV}$.

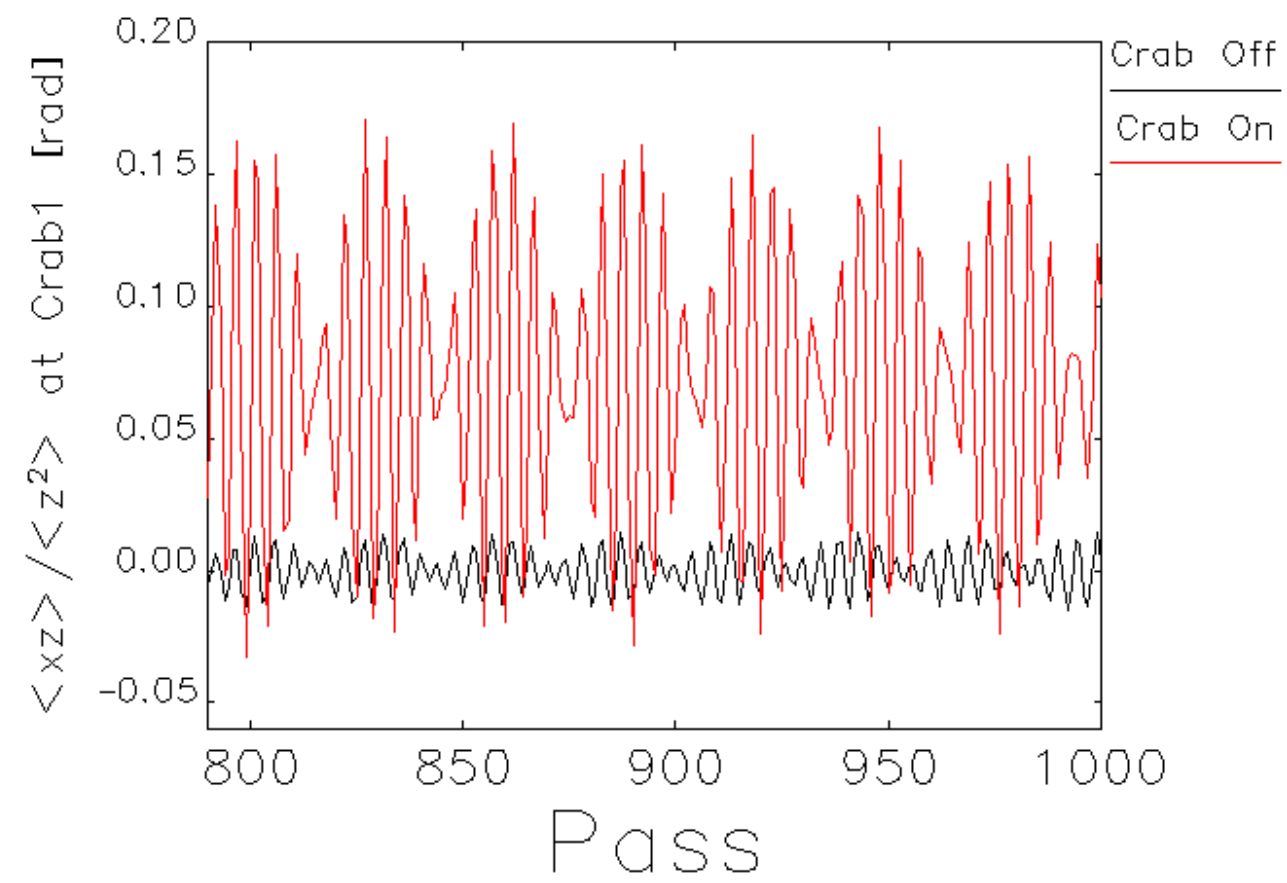

FIG. 43: Correlation of $x z$ coordinate at the location of the first crab cavity. 

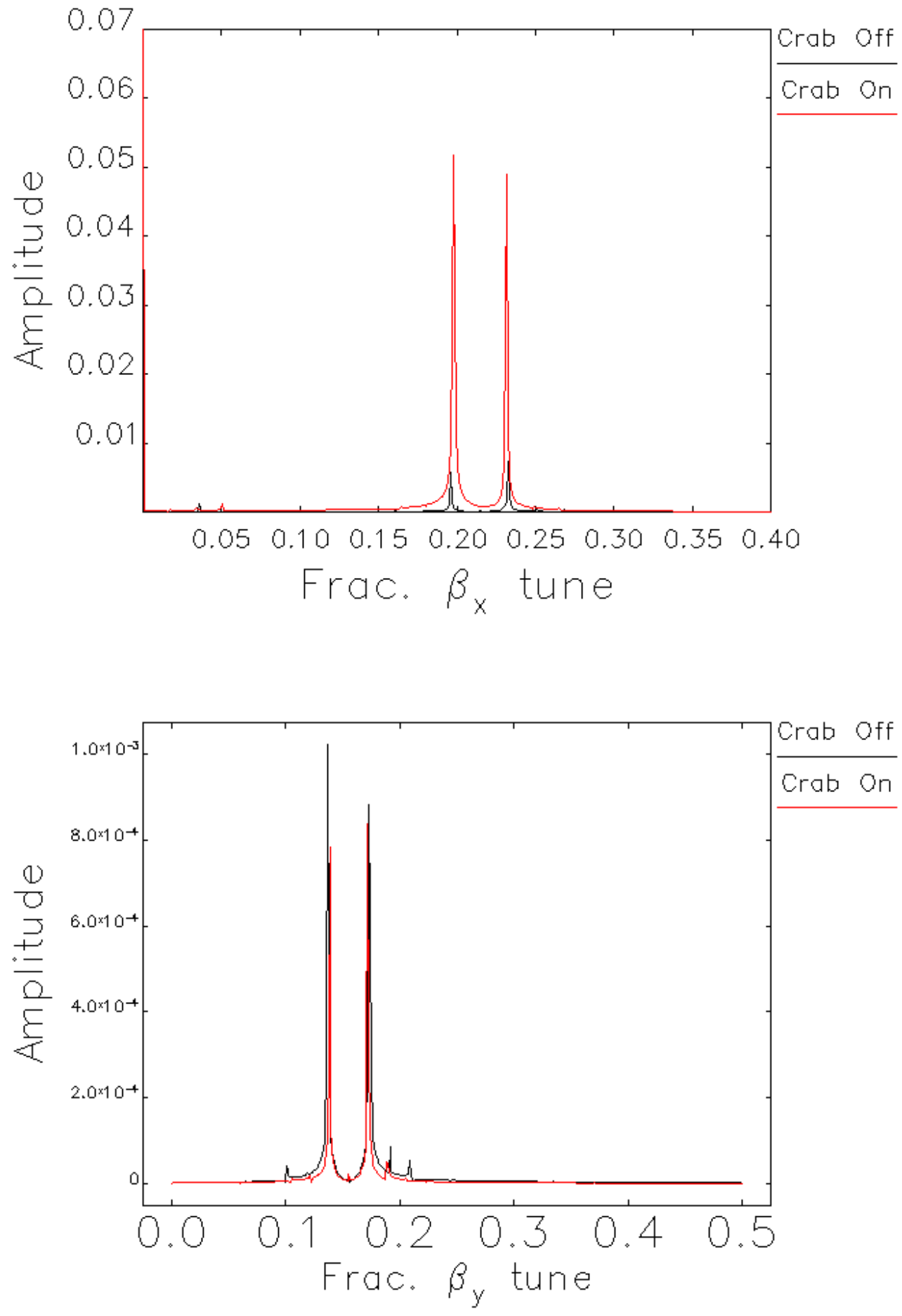

FIG. 44: Fractional horizontal and vertical betatron tunes. 


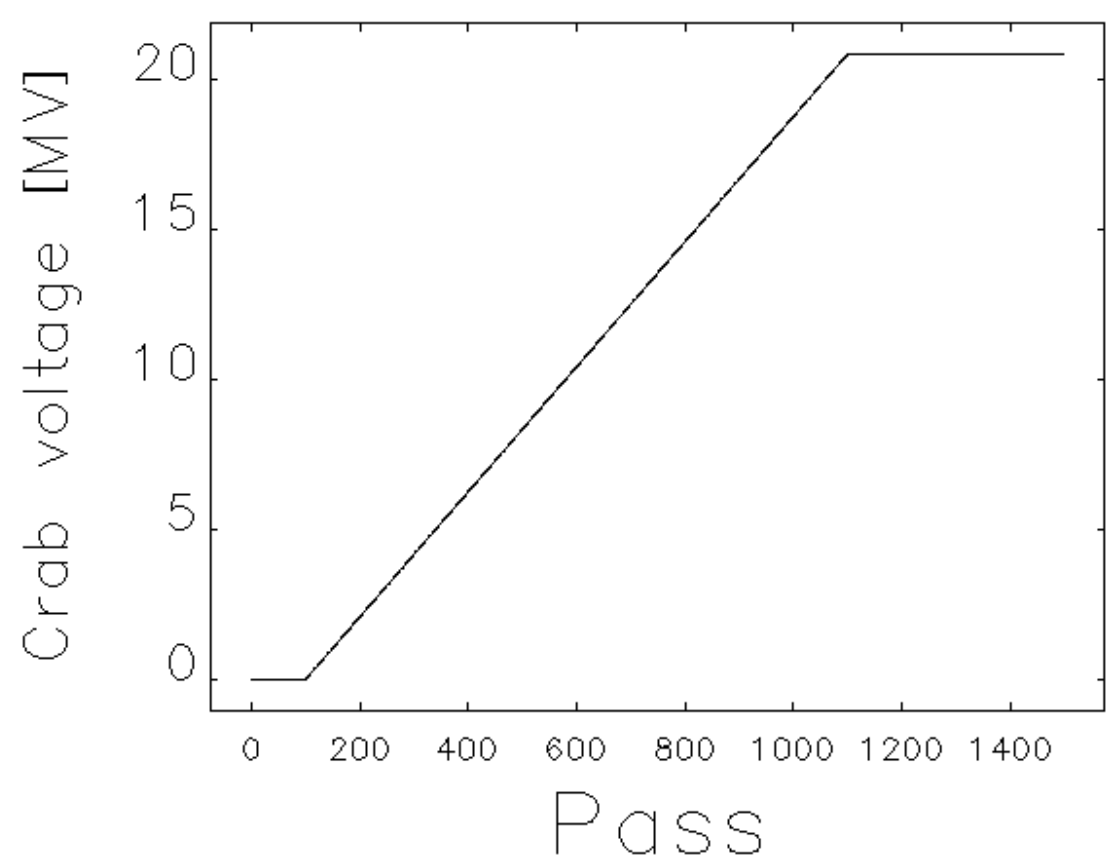

FIG. 45: The crab cavity voltage is linearly ramped over 1000 turns.

\subsection{CRAB CAVITY MULTIPOLES}

New compact crab cavity designs are very advantageous as they do not require large space in the ring. The fundamental mode is in general a non-uniform field along the axis. It is then convenient to describe the fundamental field as a multipolar expansion. In this section, we present the effect of different multipole field coefficients on the dynamics of the beam, particularly its effect on the ring dynamic aperture and report tolerance values for crab cavity multipoles.

In the design and analysis of magnets, the magnetic field can be decomposed into its multipolar terms. Such multipolar expansion can be written as [35]

$$
B(r, \phi)=B_{\mathrm{ref}} \sum_{n=1}^{\infty} a_{n}+i b_{n}\left(\frac{r}{r_{\mathrm{ref}}}\right)^{n-1} e^{i(n-1) \phi}
$$

where $B_{\text {ref }}$ is the reference magnetic field at a given radius of $r_{\text {ref }}$.

In the present analysis, we use a similar approach, with the appropriate inclusion of the time dependence for the rf fields. Detailed analytical treatments of the crab cavity multipole fields can be found in [51, 52]. Particularly, the $n$-th order multipole will produce a horizontal 


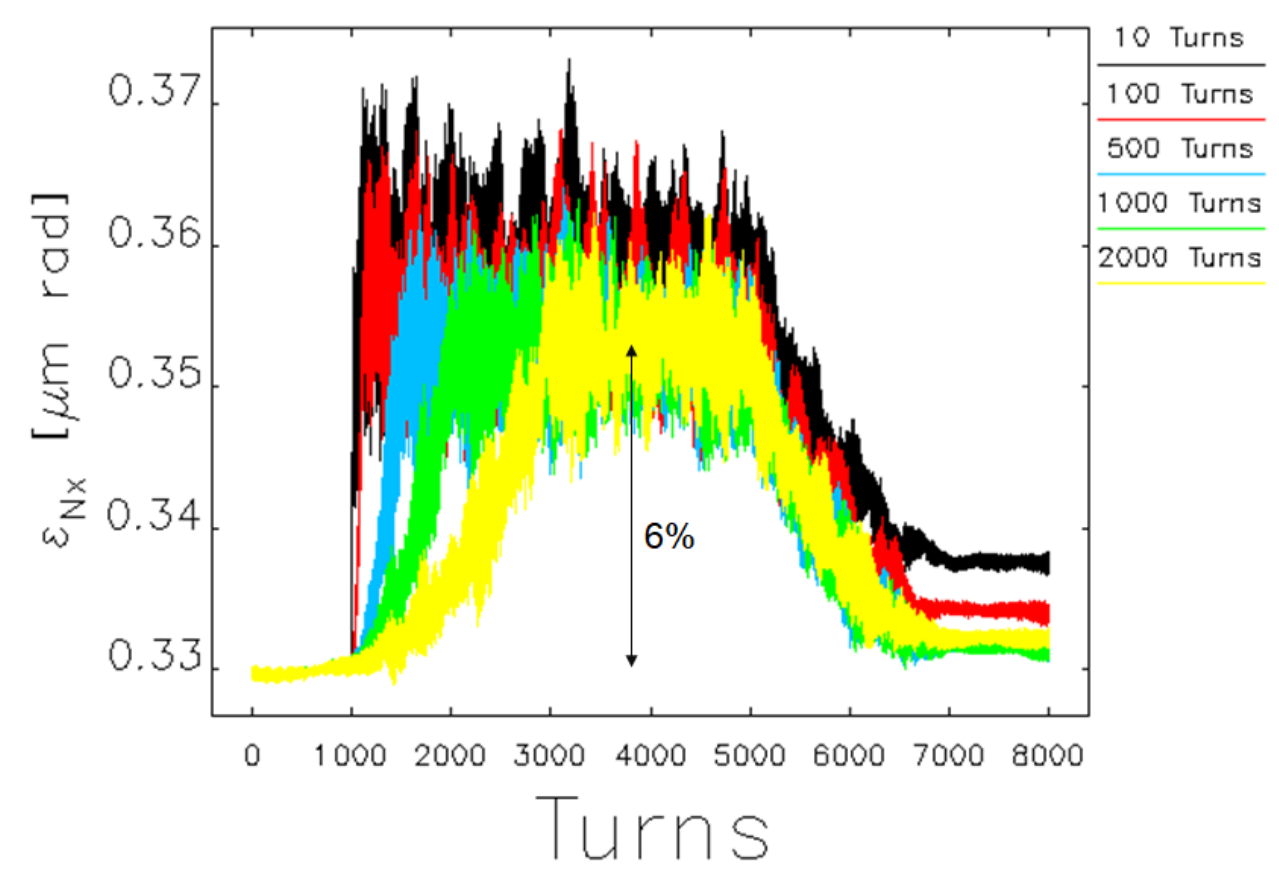

FIG. 46: Projected normalized horizontal beam emittance in the modified lattice. The growth is due to the establishment of a new matched ellipse. The voltage on the crab cavities is ramped over 10, 100, 500, 1000 and 2000 turns for comparison. Emittance can be preserved as long as the turn-on rate is longer than 500 turns.

momentum change from a horizontal displacement (assuming no vertical displacement)

$$
\Delta p_{x}^{(n)}=q x^{n-1} b_{n}
$$

with $q$ the particle charge, $x$ the horizontal position of a particle with respect to the magnet center, and $b_{n}$ are the multipole field strengths. Using a standard definition for magnets, $b_{n}$ are calculated as

$$
b_{n}=\int_{-\infty}^{\infty} B^{(n)}(z) d z, \quad\left[\frac{T}{m^{n-2}}\right] \text {, }
$$

with

$$
B^{(n)}(z)=\frac{1}{(n-1) !} \frac{\partial^{n-1} B_{y}}{\partial x^{n-1}},
$$

the $n$-th term in the field multipole expansion. A change in momentum $\Delta p_{x}^{(n)}$ will deflect particles by

$$
\Delta x_{(n)}^{\prime}=\frac{\Delta p_{x}^{(n)}}{p_{z}}=\frac{q}{\gamma m \beta c} x^{n-1} b_{n} .
$$




\section{Multipole effects on beam dynamics}

Multipole components of the crabbing electric field introduce aberrations in the ring, including a linear tune shift

$$
\Delta Q_{x, y}=\frac{1}{4 \pi} \beta_{x, y} \frac{b_{2}}{B \rho},
$$

minimum tune split from the linear coupling

$$
\Delta Q_{\min }=\frac{1}{4 \pi} \sqrt{\beta_{x} \beta_{y}} \frac{a_{2}}{B \rho},
$$

chromaticity shift

$$
\Delta \xi_{x, y}= \pm \frac{1}{4 \pi} D_{x} \beta_{x, y} \frac{2 b_{3}}{B \rho},
$$

and chromatic coupling:

$$
\frac{\partial Q_{\min }}{\partial \delta}=\frac{1}{2 \pi} \sqrt{\beta_{x} \beta_{y}} D_{y, x} \frac{2(b, a)_{3}}{B \rho}
$$

with $\beta_{x, y}$ and $D_{x, y}$ the beta and dispersion functions, $B \rho$ the magnetic rigidity of the beam and $\delta$ the relative energy spread.

\subsubsection{CRAB CAVITY WITH MULTIPOLES}

It is relevant to study the effect that multipole terms have on the beam dynamics in order to determine acceptable multipoles when designing a particular crab cavity. We use the ion ring Lattice 2 for this studies. In elegant, crab cavities with multipoles up to $n=5$, the decapole term, can be implemented using Multipole RF DeFlector (MRFDF) elements. These elements change the particles transverse momentum by

$$
\Delta p_{x}=\frac{e}{m \omega} \sum_{n=1}^{5} n b_{n} x^{n-1} \cos \phi_{n}
$$

with $m$ the particle mass, $\omega$ the angular rf frequency, $\phi_{n}$ the phase of the multipole term and the particle's deflection is given by $\Delta x^{\prime}=\Delta p_{x} / p_{z}$. In this expression, $b_{n}$ has units $V / m^{n}$. It is useful to have an equivalent expression in units of Tesla $[\mathrm{T}]$, as this follows the convention in the treatment of magnets; from Eqs. (116) and (124), a relation

$$
b_{n}\left[\frac{V}{m^{n}}\right]=b_{n}\left[\frac{T}{m^{n-2}}\right] \cdot \frac{\omega}{n}
$$

can be determined. Using MRFDF elements, different multipoles can be assigned independent amplitude and phase values. Since the multipoles refer to the expansion coefficients of the field providing the crab kick, it is understood that they are all on-phase. 
In Section 5.3, we can get a sense of the expected aberrations due to multipole coefficients through analytical expressions. We now investigate more accurate effects by using our model of the ion ring and including cavities with non-zero multipolar terms. Furthermore, the multipole coefficients used correspond to actual cavity designs modeled in CST Microwave Studio [5].

\subsubsection{DYNAMIC APERTURE CALCULATION WITH ELEGANT}

A more accurate way of estimating the effect of the cavity multipoles is through their effect on the ring dynamic aperture. The way elegant calculates the dynamic aperture of a ring is by partitioning the configuration space into a number $n$ of lines or rays coming out of the origin as shown in Fig. 47 .

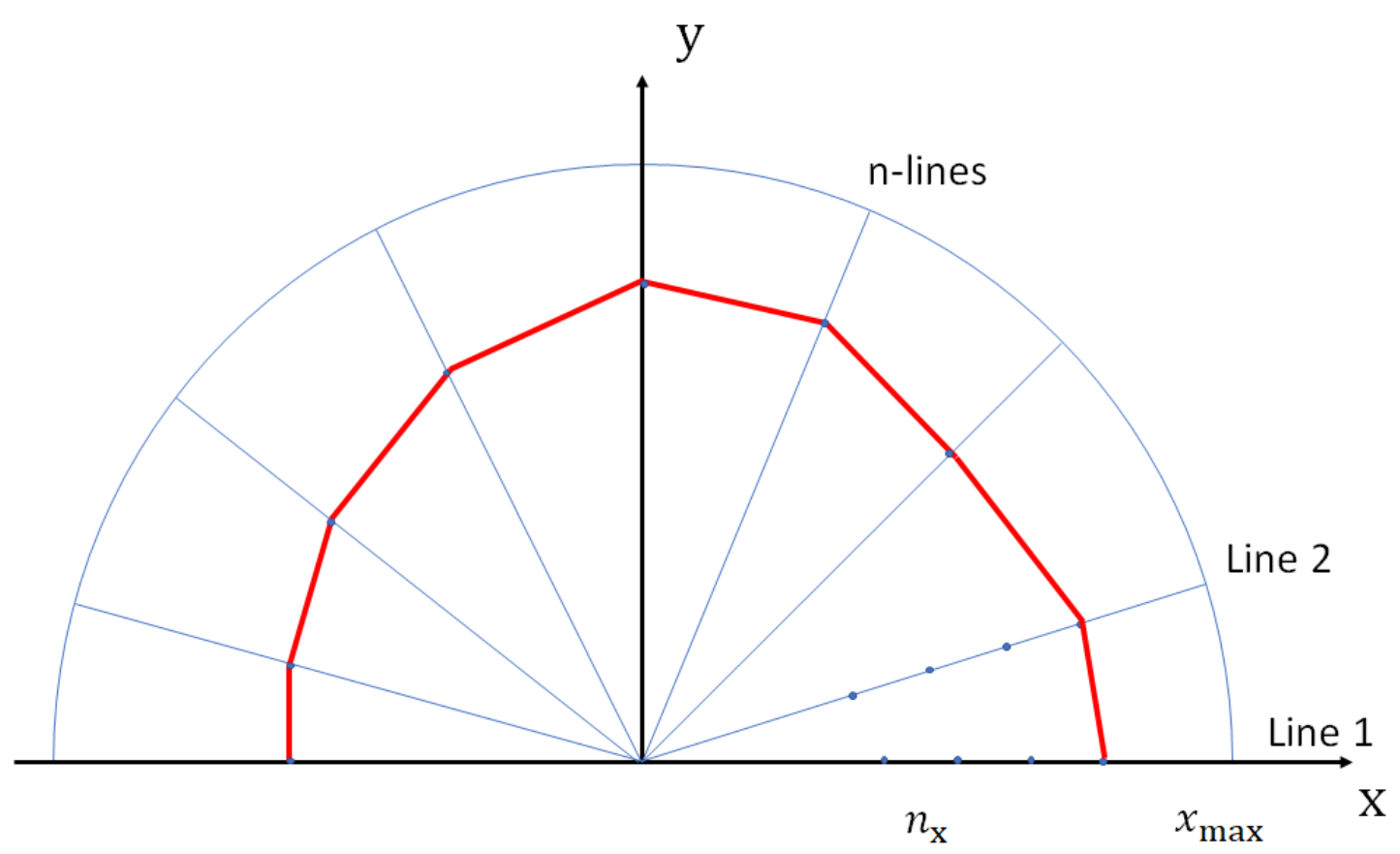

FIG. 47: Calculation of the ring dynamic aperture using elegant.

Each of the rays is then divided into $n_{x}$ intervals up to a selected $x_{m a x}$ value. Starting from the first ray on the axis, a particle is placed at the $x_{\max }$ location and tracked through the ring for $N$ turns, typically a few thousand turns. If the particle survives after the turns, it is said to be stable, and the $x$-value recorded. If it does not survive, however, a new particle is placed in the following point and tracked again. This same process is repeated several times, effectively scanning through the $n_{x}$ partitions in the first ray until a point is found where the particle is stable. In the same way, a particle is now scanned through the 
$n_{x}$ partitions of the second ray, following a counterclockwise order and until a stable point is found. The process repeats for all the $n$-lines rays, and the dynamic aperture is determined by the area enclosed by all the stable points. Vertical symmetry of the ring allows us to calculate only half of the vertical plane, and although a large number of rays and partitions will yield better accuracy, it comes as a trade-off on computing time.

\subsubsection{EFFECT OF MULTIPOLES ON THE RING DYNAMIC APERTURE}

The dynamic aperture (DA) of $100 \mathrm{GeV}$ proton beam is investigated when crab cavities with multipole field content are placed at horizontal CCS locations in the ion ring. The DA is evaluated over 2000 turns and scanned in the $x y$ plane along 55 rays. Strong beam cooling emittance values are assumed, where $\gamma \epsilon_{x}=0.35$ and $\gamma \epsilon_{y}=0.07 \mu \mathrm{m} \cdot \mathrm{rad}$. These values correspond to transverse bunch sizes $\sigma_{x}=0.121 \mathrm{~mm}$ and $\sigma_{y}=0.154 \mathrm{~mm}$ at the beginning of the lattice where the DA is determined. As in the previous section, the starting point is chosen outside the crab region, in a dispersion-free location with $\alpha_{x}=\alpha_{y}=0$ and $\beta_{x}=4.885$ $\mathrm{m}$ and $\beta_{y}=32.681 \mathrm{~m}$. We first study the effect of a crab cavity linear kick, i.e., using the RFDF element with no multipoles. Figure 48 shows the beam DA of the ion ring when the crabbing voltage is turned off and on. No considerable effect is observed in the Dynamic Aperture of the ion ring.

\section{Crab cavity multipoles}

Preliminary crab cavity design concepts for JLEIC have been reported in [32, 33]. In this contribution, multipoles corresponding to different crab cavities (1-cell, 3-cell and squashed elliptical) are used to estimate the impact on the ring DA. The cavities are shown in Fig. 49. We also study two different rf-dipole crab cavity inner pole geometries: flat and curved, which are shown in Fig. 50. The normalized multipole components and its resulting DA are presented in Table 7.

The resulting DA are presented for the rf-dipole cavities in Fig. 52 and for the squashed elliptical in Fig. 53. These results show that both 1-cell and 3-cell cavity designs lie within tolerance. The resulting DA obtained with the 60 and $70 \mathrm{~mm}$ aperture cavities appears to be in agreement. The only significant variation is suggested for the different pole tips. Curved pole tips were initially motivated to study a smaller sextupole coefficient, but the results suggest that by making $b_{3}$ smaller, the $b_{5}$ term contributes to limit the DA. 


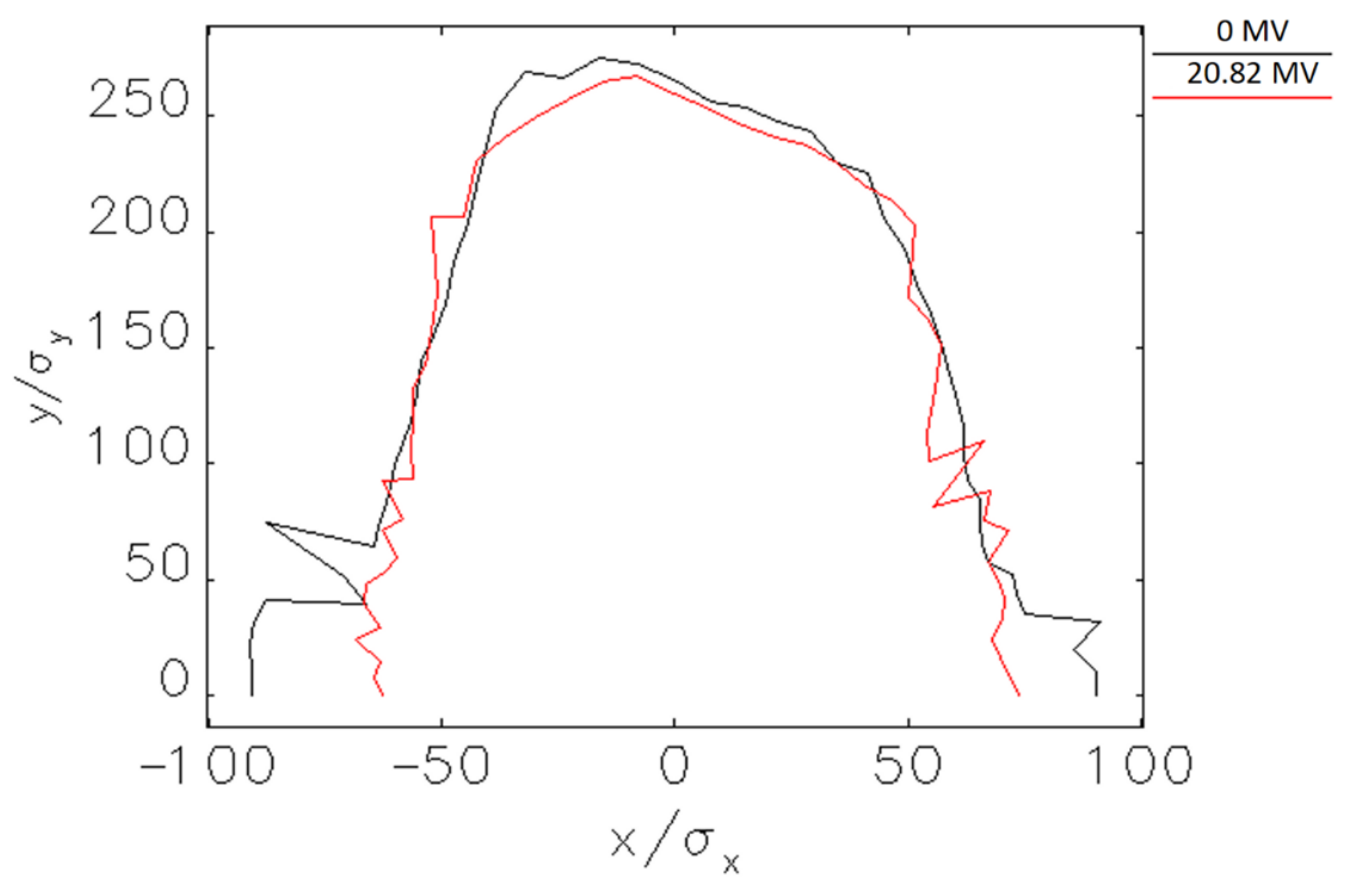

FIG. 48: Beam DA of the ion ring with the crab cavity voltage set to 0 and $20.82 \mathrm{MV}$ for comparison. Only a linear kick is considered in this case.

\section{Multipole tolerance}

Using the tools of the DA estimation, we can define tolerances on the multipoles to get a quick sense on whether a particular geometry will be acceptable or not. We increase each multipole independently until the resulting DA is $\sim \pm 50 \sigma_{x}$. Table 8 presents tolerances on the multipoles of a multi-cell rf-dipole crab cavity with flat poles, as defined by the signs in Table 7. When combined into a "tolerance" cavity, they reduce the ring DA to $\pm 46.2 \sigma_{x}$, and is used for comparison with other crab cavity designs. Figure 51 shows the resulting DA for all the crab cavity geometries considered. 

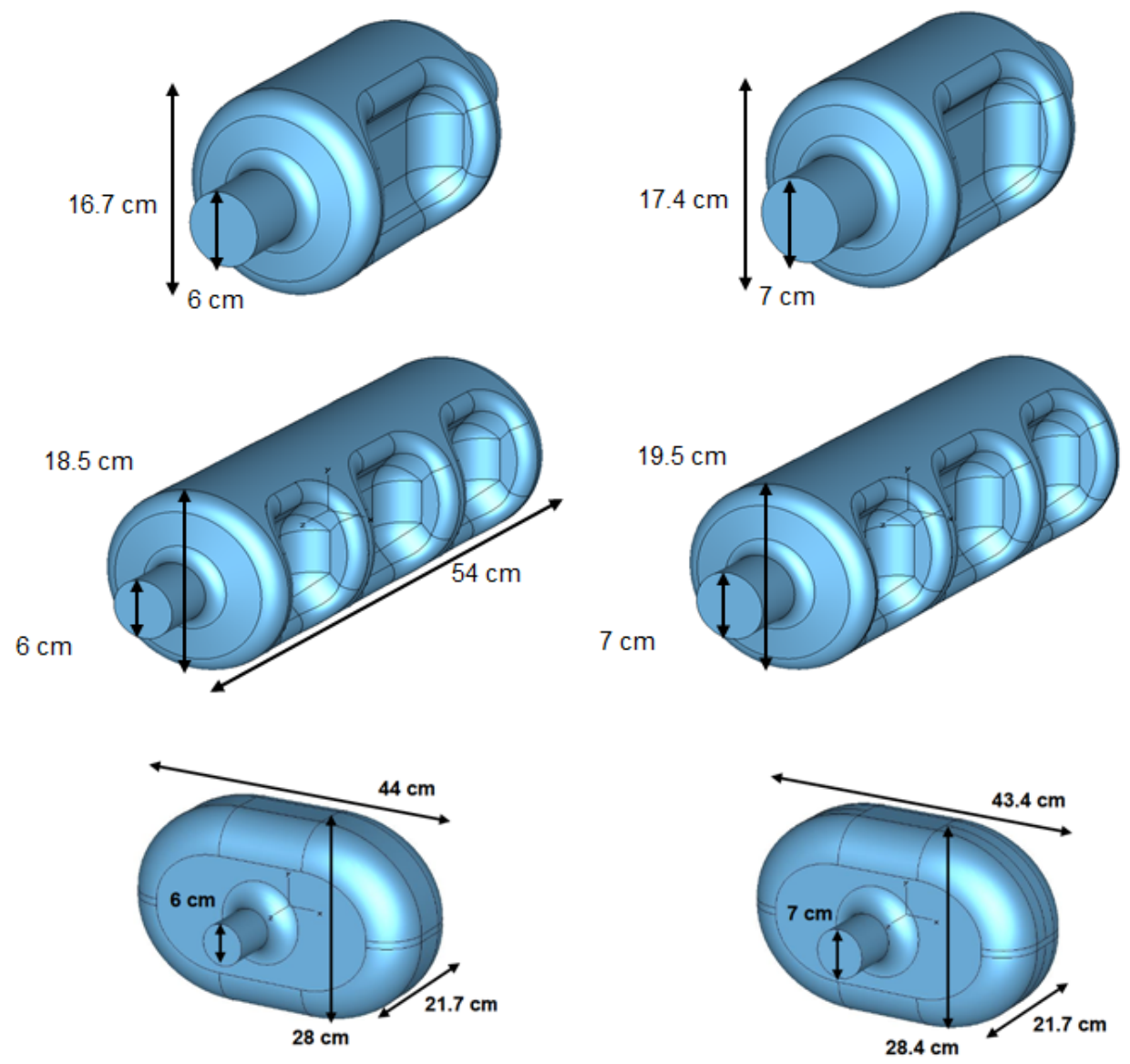

FIG. 49: Crab cavity concepts for JLEIC used for multipole studies. Shown are single-cell, 3-cell crab and squashed elliptical cavity designs with $60 \mathrm{~mm}$ and $70 \mathrm{~mm}$ apertures.
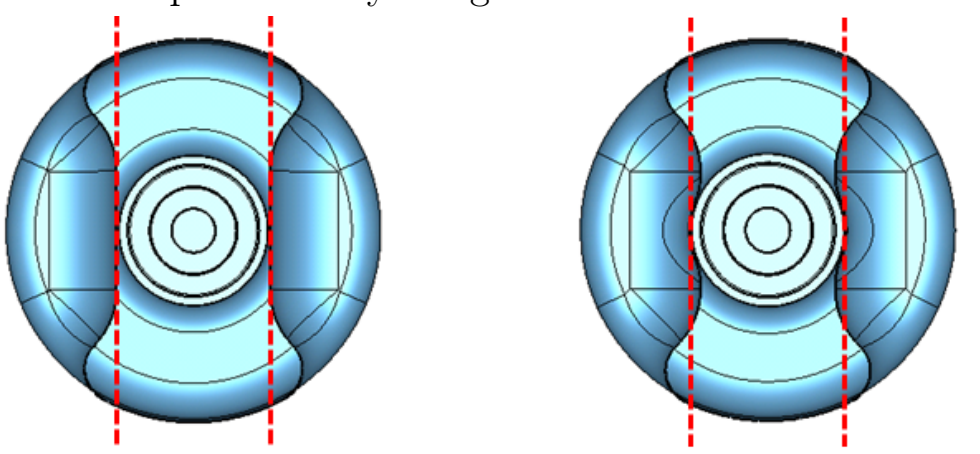

FIG. 50: Different inner pole geometries used on the single-cell and 3-cell rf-dipole crab cavities. Left figure shows a flat pole tip, right figures shows a curved pole tip. 
TABLE 7: Normalized multipole coefficients for 1-cell, 3-cell and squashed elliptical crab cavities. The last row shows the resulting dynamic aperture.

\begin{tabular}{|c|c|c|c|c|c|c|c|c|c|c|}
\hline \multirow{3}{*}{$b_{n}\left[\frac{m T / m^{n-2}}{1 M V}\right]$} & \multicolumn{4}{|c|}{ 1-cell } & \multicolumn{4}{|c|}{ 3-cell } & \multicolumn{2}{|c|}{ Squashed } \\
\hline & \multicolumn{2}{|c|}{$60 \mathrm{~mm}$} & \multicolumn{2}{|c|}{$70 \mathrm{~mm}$} & \multicolumn{2}{|c|}{$60 \mathrm{~mm}$} & \multicolumn{2}{|c|}{$70 \mathrm{~mm}$} & $60 \mathrm{~mm}$ & $70 \mathrm{~mm}$ \\
\hline & Flat & Curved & Flat & Curved & Flat & Curved & Flat & Curved & - & - \\
\hline$V_{t}[\mathrm{MV}]$ & & & & & & 1.0 & & & & \\
\hline$b 1$ & 3.33 & 3.33 & 3.33 & 3.33 & 3.33 & 3.33 & 3.33 & 3.33 & 3.33 & 3.33 \\
\hline$b_{2}\left[\times 10^{-4}\right]$ & -5 & -5 & -5 & -6 & -3 & -4 & -3 & $-8 \times 10^{-5}$ & $-1.7 \times 10^{-5}$ & -12 \\
\hline$b_{3}$ & 853.2 & -293.3 & 797.5 & -1.64 & 697.1 & 45.3 & 610 & 35.1 & 77.9 & 76.4 \\
\hline$b_{4}$ & 1.55 & 1.59 & 1.2 & 1.35 & 0.92 & 1.16 & 0.63 & $-2 \times 10^{-5}$ & $-5 \times 10^{-6}$ & 2.56 \\
\hline$b_{5}\left[\times 10^{5}\right]$ & -1.2 & -13 & -0.44 & -7.5 & -1.1 & -9.1 & -5.4 & -5.7 & $-391.9 \times 10^{-5}$ & -0.028 \\
\hline$b_{6}$ & -3544.4 & -3640.7 & -1866.0 & -2143.2 & -2092.5 & 0.0013 & -1002.7 & -0.024 & -0.012 & -4065.1 \\
\hline$b_{7}\left[\times 10^{7}\right]$ & -4.2 & -44.0 & -1.5 & -26.0 & -4.1 & -35.0 & -1.4 & -10.9 & -0.18 & -0.12 \\
\hline $\mathrm{DA}\left[\sigma_{x}\right]$ & \pm 45.9 & \pm 24.8 & \pm 46.6 & \pm 31.7 & \pm 44.1 & \pm 27.8 & \pm 47 & \pm 30.9 & \pm 45.9 & \pm 45.8 \\
\hline
\end{tabular}


TABLE 8: Normalized multipole coefficients that reduce the horizontal DA to $\pm 50 \sigma_{x}$.

\begin{tabular}{|l|c|}
\hline $\mathbf{b}_{\mathbf{n}}\left[\frac{m T / m^{n-2}}{1 M V}\right]$ & Maximum Tolerance \\
\hline$b_{1}$ & 3.33 \\
\hline$b_{2}$ & -16.08 \\
\hline$b_{3}$ & 217.12 \\
\hline$b_{4}$ & 321.67 \\
\hline$b_{5}$ & $-0.8 \times 10^{5}$ \\
\hline DA $\left[\sigma_{x}\right]$ & \pm 46.2 \\
\hline
\end{tabular}

It is important to mention two things regarding the multipoles of crab cavities. First, the JLEIC ion ring DA is limited to $\pm 10 \sigma$ by the multipole fields of the final focusing quadrupoles (FFQ) [53]. Therefore, the results presented in this contribution suggest that none of the crab cavity designs considered would be a limiting factor to the ring DA. A complete JLEIC collider DA simulation should include effects from both FFQ multipoles and crab cavity multipoles. Second, a more accurate model of the crab cavity multipoles will involve a finite-length cavity, where a field map can be used, rather than a multipole expansion.

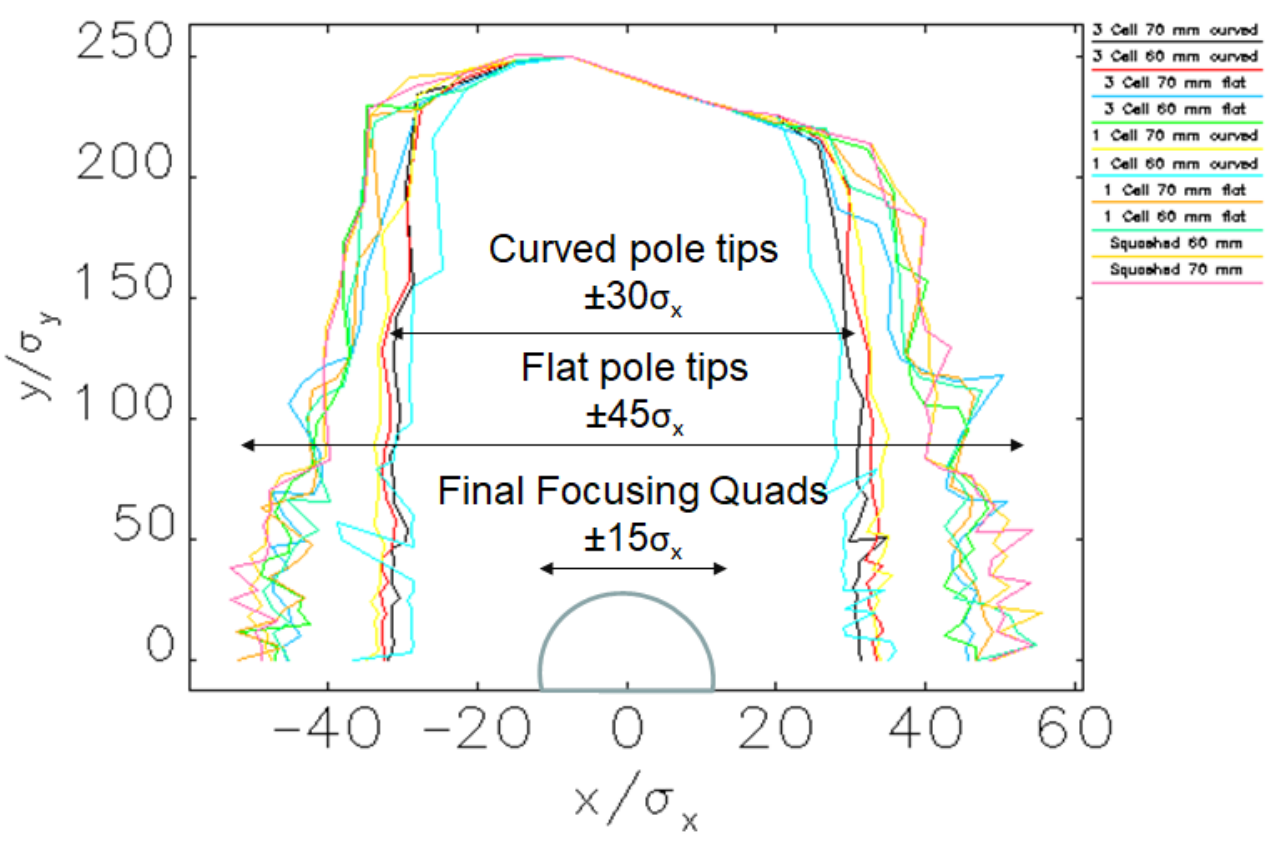

FIG. 51: The resulting DA corresponding to the crab cavity designs shown in Fig. 49 . 


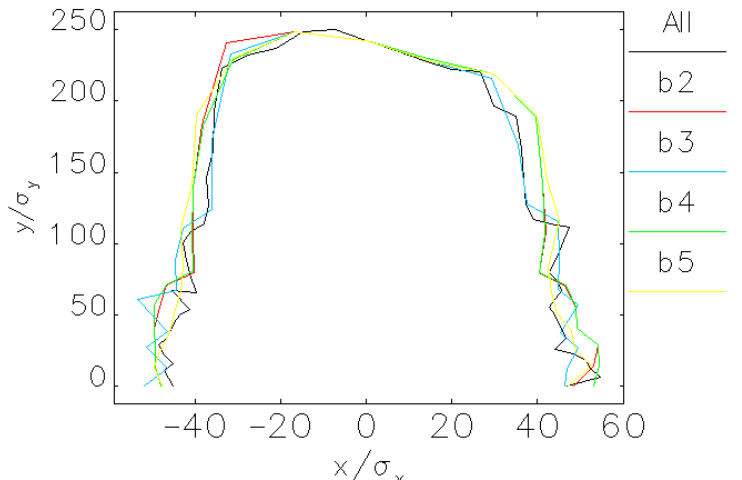

1 Cell $60 \mathrm{~mm}$ flat

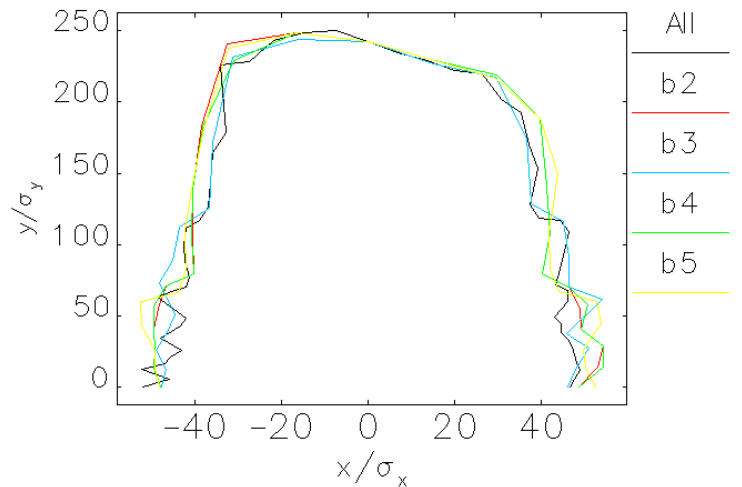

1 Cell $70 \mathrm{~mm}$ flat

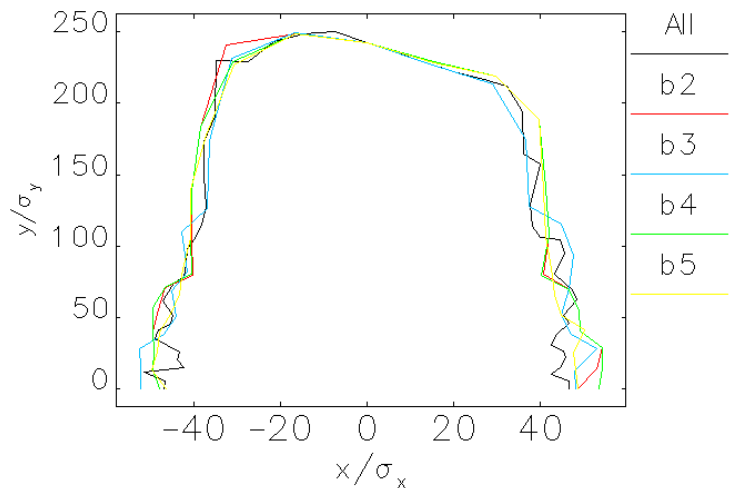

3 Cell $60 \mathrm{~mm}$ flat

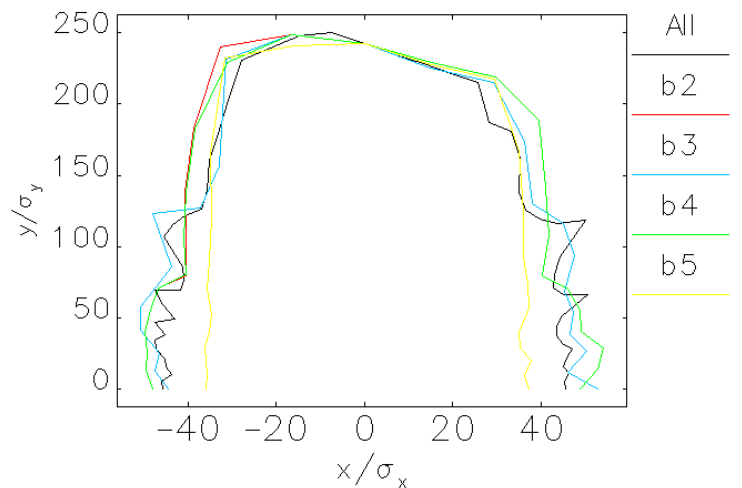

3 Cell $70 \mathrm{~mm}$ flat

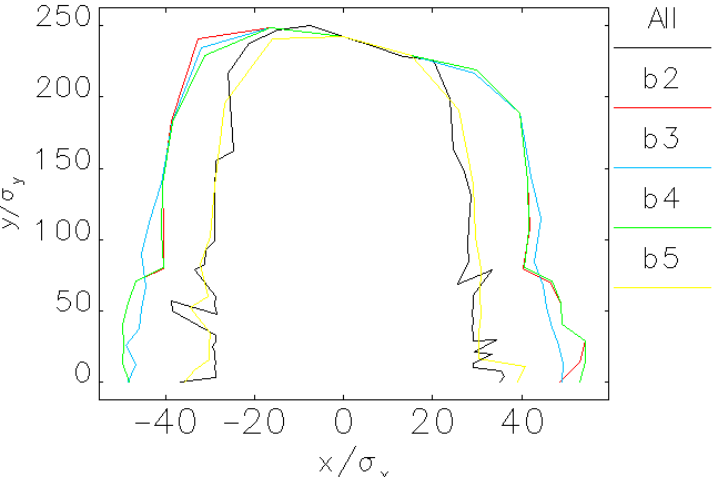

1 Cell $60 \mathrm{~mm}$ curved

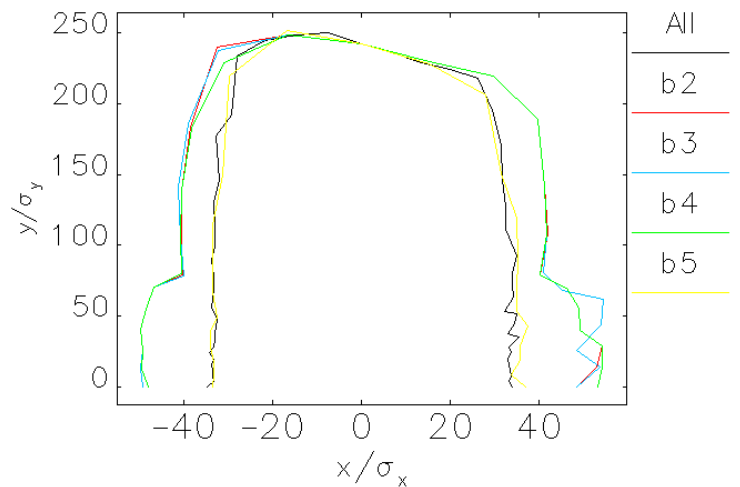

1 Cell $70 \mathrm{~mm}$ curved

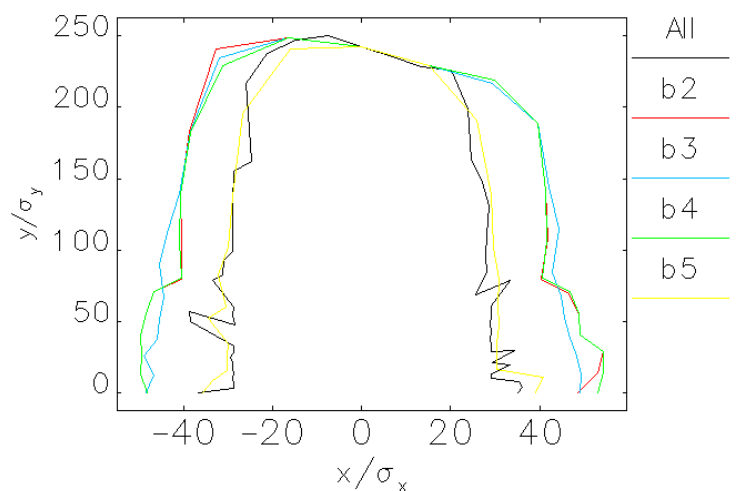

1 Cell $60 \mathrm{~mm}$ curved

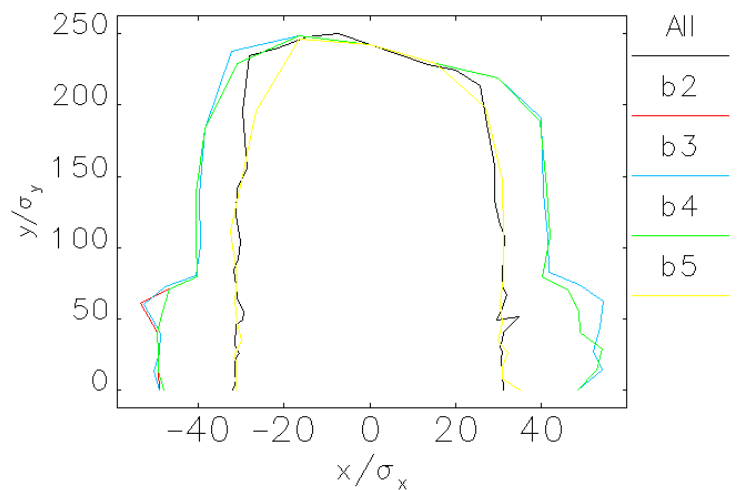

3 Cell $70 \mathrm{~mm}$ curved

FIG. 52: Resulting DA from multipole coefficients of single-cell and 3-cell rf-dipole crab cavity concepts with different apertures and pole tip geometries. 

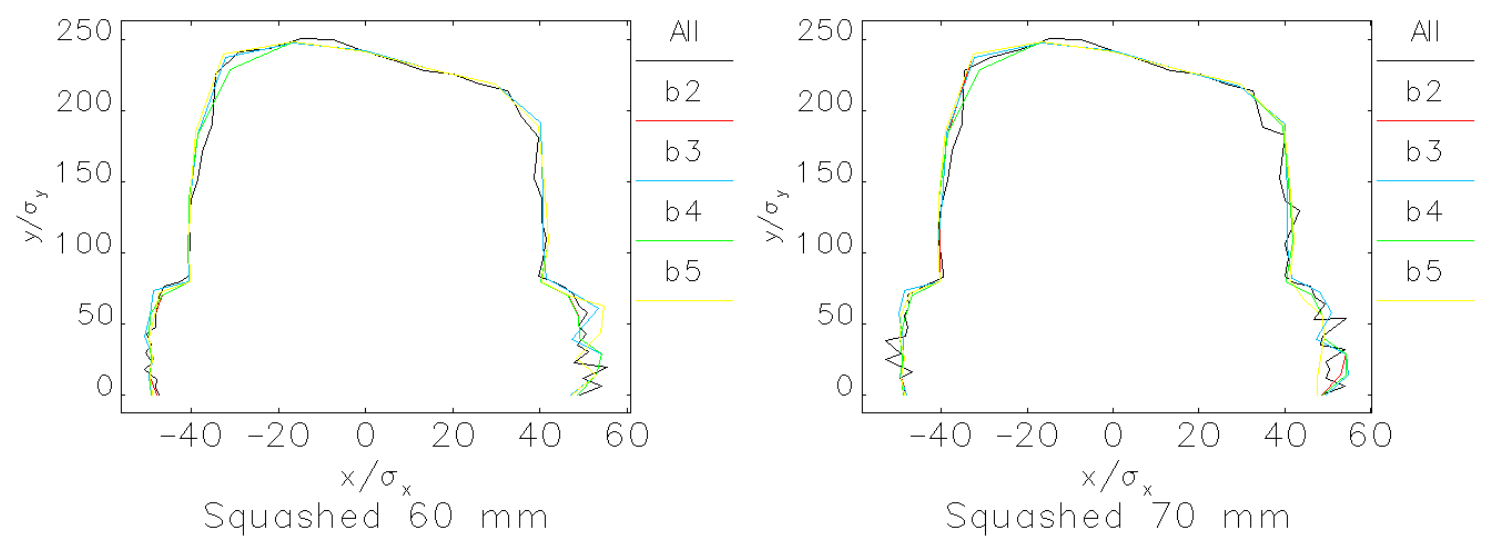

FIG. 53: Resulting DA from multipole coefficients of squashed cavities with $60 \mathrm{~mm}$ and 70 $\mathrm{mm}$ apertures.

\subsection{RF NOISE}

Noise in the amplitude and phase of the crab cavity voltage needs to be controlled in order to avoid degradation of the beam emittance, particularly of the cooled ion beam.

\subsubsection{VOLTAGE AMPLITUDE ERROR}

Any variation on the amplitude of the crab voltage introduces an extra error in the tilt at the interaction point as illustrated in Fig. 54. In a local scheme, synchro-betatron coupling may be excited as the crabbing is not exactly canceled. A sufficient requirement on the voltage amplitude noise is for the residual tilt angle to be much smaller than the geometric bunch diagonal [13],

$$
\frac{\Delta V}{V} \ll \frac{1}{\tan \left(\frac{\phi_{\text {cross }}}{2}\right)} \frac{\sigma_{x}^{*}}{\sigma_{z}} .
$$

Assuming a JLEIC bunch with $\sigma_{x}^{*}=18 \mu \mathrm{m}$ and $\sigma_{z}=1.2 \mathrm{~cm}$, Eq. (126) gives $\Delta V / V \ll 5 \%$. In elegant, we can introduce different noise levels in the ring and evaluate the long-term stability. First, we fully ramp the cavity voltage over 1000 turns, then introduce a fractional rms noise of $0.1 \%, 0.05 \%$ and $0.01 \%$ in both crab cavities. For each noise set point, we track a bunch of 100 particles over $5 \times 10^{4}$ turns. Figure 55 presents the distribution of the produced bunch angle at IP corresponding to each noise value.

For $\Delta V / V=0.1 \%$, we get a $\sigma=0.85 \mathrm{mrad}$ standard deviation on the produced tilt; for $\Delta V / V=0.05 \%$, there is $\sigma=0.42 \mathrm{mrad}$ and for $\Delta V / V=0.01 \%$ there is $\sigma=0.15 \mathrm{mrad}$. In Fig. 56, the beam emittance corresponding to different noise values is tracked over number of 


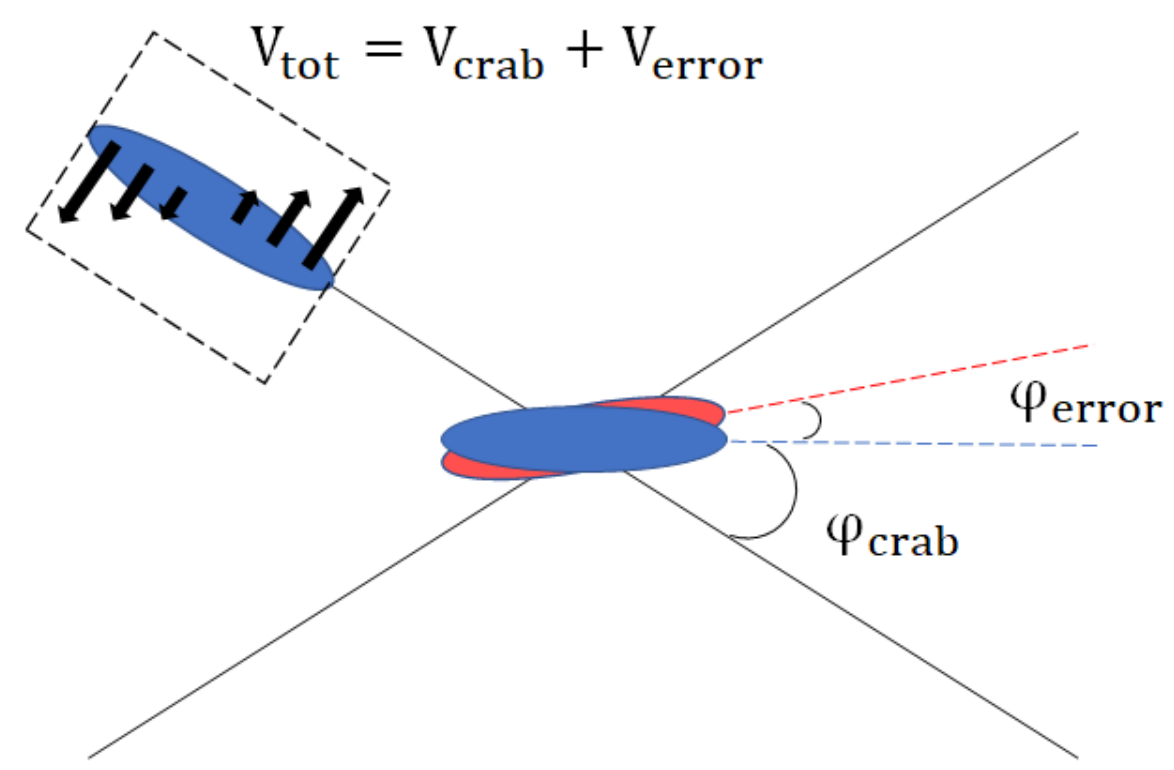

FIG. 54: An error in the crab cavity voltage amplitude produces an error in the tilt angle at the interaction point.

turns. Based on these, we recommend controlling the voltage amplitude noise below $0.01 \%$ for negligible impact on emittance.

\subsubsection{VOLTAGE PHASE ERROR}

In a similar way, a phase or timing error produces a transverse offset, see Fig. 57, of the bunch at the IP

$$
\Delta x_{\mathrm{IP}}=\frac{c \phi_{\mathrm{cross}}}{\omega_{\mathrm{rf}}} \Delta \phi
$$

In this case, the tolerable offset is much smaller than the transverse beam size at IP [13],

$$
\Delta \phi \ll \frac{\omega_{\mathrm{rf}} \sigma_{\mathrm{x}}^{*}}{c \tan \frac{\phi_{\mathrm{cross}}}{2}} .
$$

With JLEIC bunch parameters, this condition is $\Delta \phi \ll 20 \mathrm{mrad} \simeq 1.1 \mathrm{deg}$. We introduce different levels of noise in the phase of the cavity voltage and track over many turns to evaluate its effect. In Fig. 58 we show the beam emittance as a function of turns assuming 0.5, 1 and 5 mdeg phase noise levels. This suggest that the phase jitter needs to be controlled to less than 1 mdeg for negligible effect on the beam emittance. Following Eq. (127), a 1 mdeg phase shift produces a $0.04 \mu \mathrm{m}$ transverse offset at IP, about $0.2 \%$ of the bunch size. 


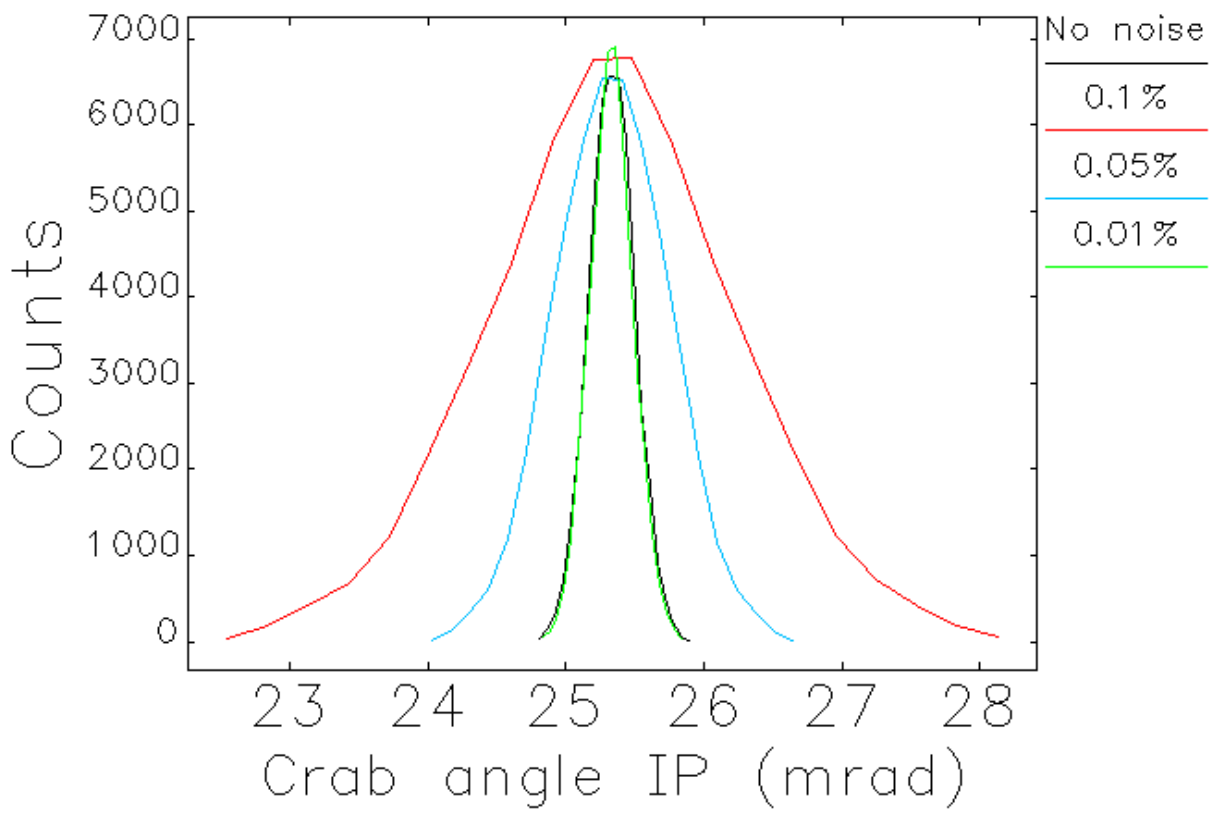

FIG. 55: Distribution of the crab angle at IP over $5 \times 10^{4}$ turns. Fractional rms noise $\Delta V / V=0.1 \%, 0.05 \%$ and $0.01 \%$ are assumed on the amplitude of the crab cavity voltage.

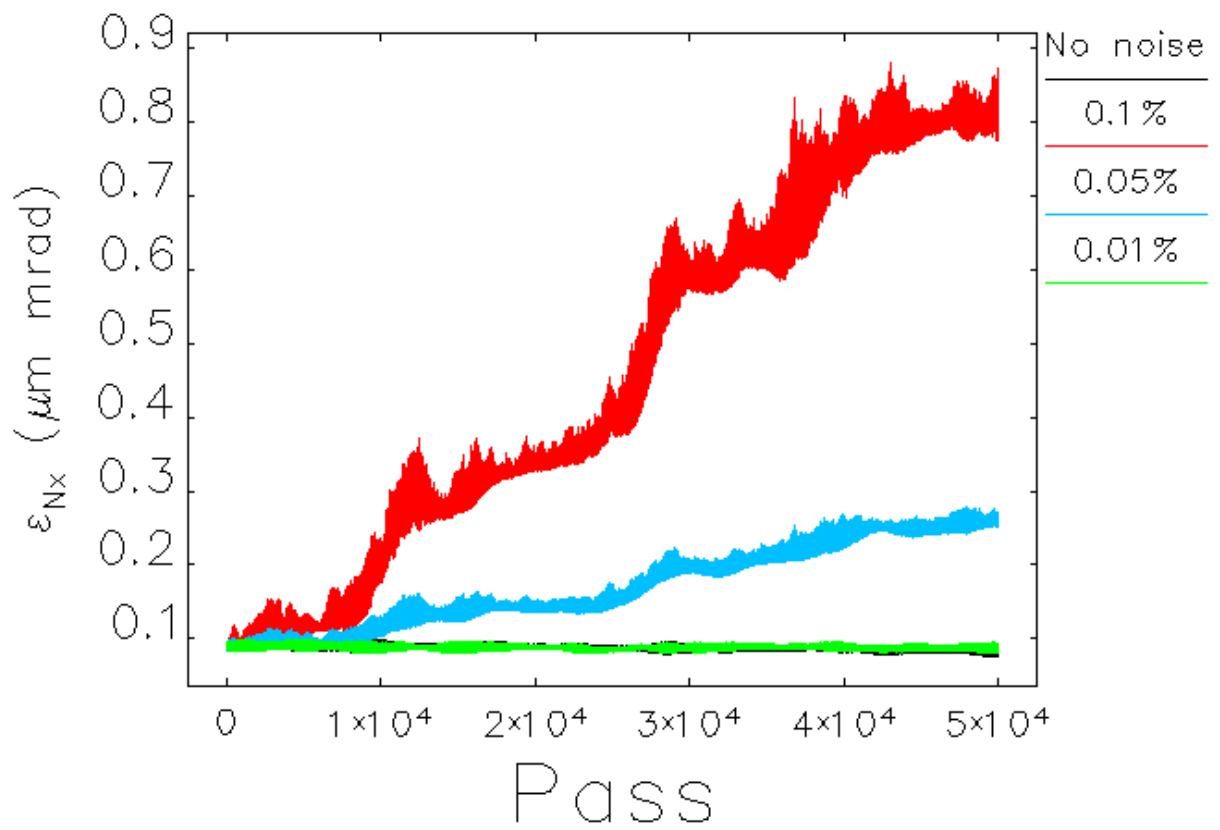

FIG. 56: Emittance increase due to different noise levels in the amplitude of the crab cavity voltage. 


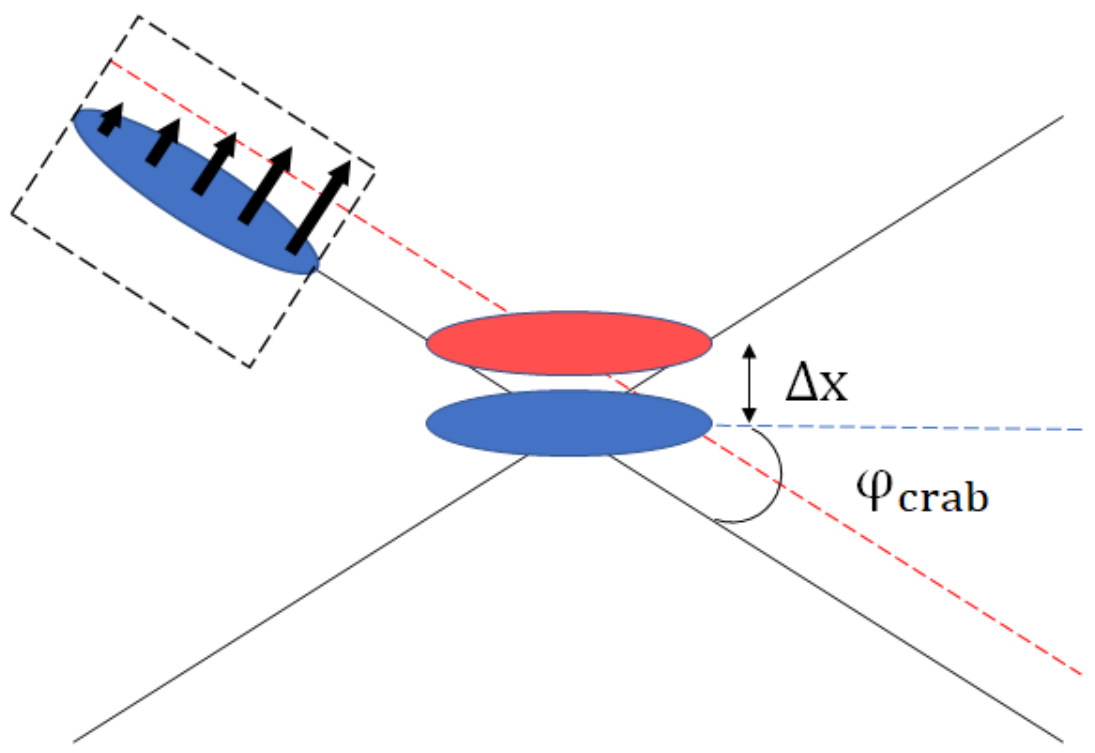

FIG. 57: A crab cavity voltage phase error, or bunch arrival time error, produce a transverse offset of the bunch at the IP.

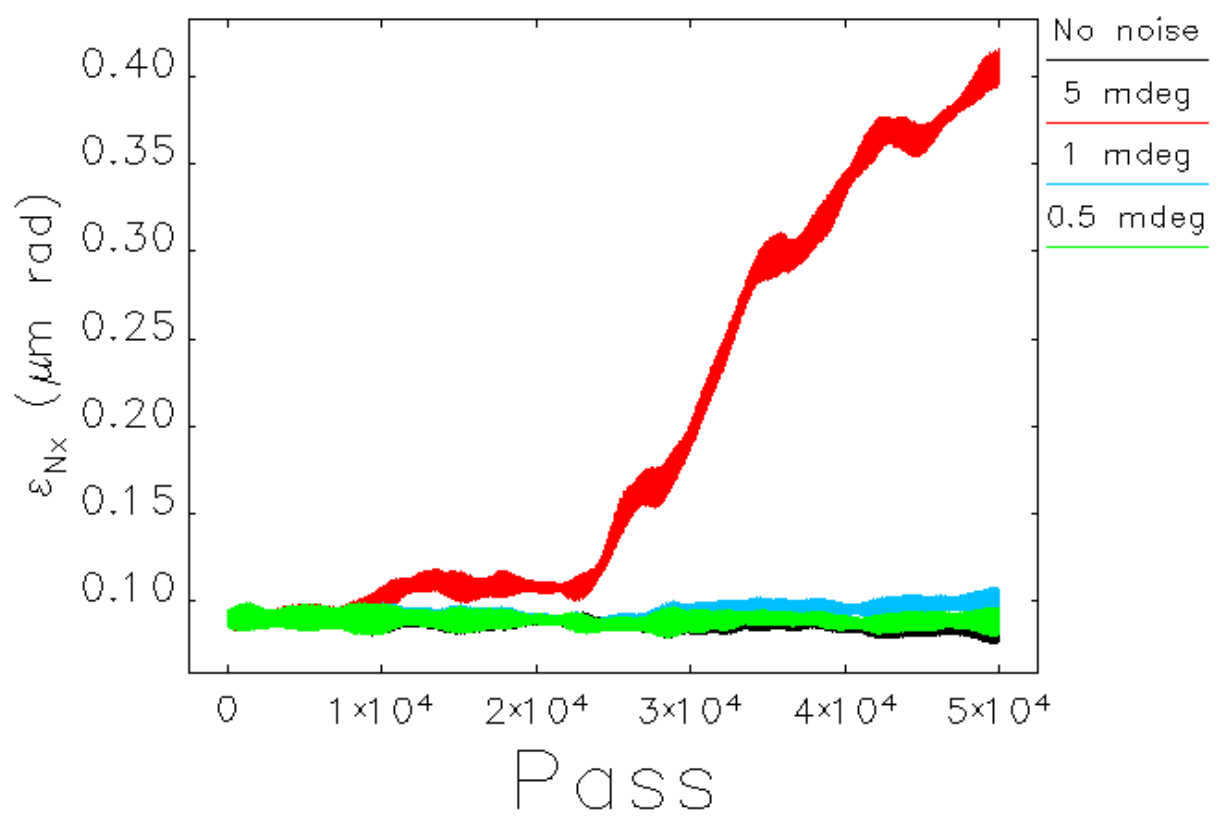

FIG. 58: Beam emittance as a function of turns for different phase noise levels in the crab cavity voltage. 


\section{CHAPTER 6}

\section{HIGHER ORDER MODES}

Up until this point, the discussion of the JLEIC crabbing system has focused on the effects exerted on the beam by the crab cavity. In this chapter, we now consider the effect the beam has on the crab cavity: the high order modes (HOMs) excited in the crab cavity by a passing beam. Two main considerations motivate the analysis of the cavity HOMs. First, HOMs can drive coupled bunch instabilities (CBI) of the beam and second, the excited HOM power can become an excessive power load for the cryogenic bath. Proper design of HOM couplers ensures safe extraction of the HOM power. In Section 6.1 we first discuss the concept of wakefield and the impedance, which helps to understand how the beam "sees" the cavity in the ring. Section 6.2 discusses the HOMs of the crab cavity. In Section 6.3 we

present the coupled-bunch instabilities and perform calculations using parameters from the ion ring and longitudinal HOMs of 2-cell and 3-cell RFD crab cavities. Finally in Section 6.4 we give an estimation of the HOM power generated in the crab cavities by JLEIC type bunches. [54] [55]

\subsection{WAKEFIELD AND IMPEDANCE}

The beam traveling along the ring can induce electromagnetic fields in different components of a particle accelerator ring. From the conservation of energy principle, any charged particle traveling in the beam pipe must leave energy behind whenever the boundary conditions are modified, for example, whenever the cross section of the beam pipe changes, or when traveling through a non-conducting boundary. The energy left behind by the beam is known as a wakefield. Among the ring elements that are common places for the beam to excite wakefields are: bellows, beam position monitors, resistive beam pipes, tapers, and rf cavities. Here our focus will be on the wakefields induced by the beam on resonant elements, specifically on the crab cavities for JLEIC.

Wakefields can be of two types: short and long range. In the former, the wakefield decays very fast and usually acts within the same bunch, space charge in low-energy beams and microwave instabilities are realizations of the effect of a short wakefield in the ring. Another good example, a resistive wall, also produces short-range wakefields. In the long-range type, wakefields can linger for long times, thus being able to act on subsequent bunches. 
Wakefields are a time-domain quantity. The Fourier transform of the wakefield into frequency domain is called the impedance. Short range wakefields become a broadband impedance, while long range wakefields become narrow-band impedance. These are usually high- $Q$ resonant elements like accelerating or crabbing cavities.

\subsubsection{MODEL OF A SINGLE CAVITY RESONANT MODE}

The longitudinal wakefield of a resonant mode characterized by a frequency $\omega_{r}$, impedance $R_{s}$ and quality factor $Q$ is [35]

$$
W(t)= \begin{cases}0, & \text { for } t<0 \\ 2 q_{1} k e^{\gamma t}\left(\cos \omega_{1} t-\frac{\gamma}{\omega_{1}} \sin \omega_{1} t\right), & \text { for } t \geq 0\end{cases}
$$

with $\gamma=\omega_{r} / 2 Q, \omega_{1}=\omega_{r} \sqrt{1-1 / 4 Q^{2}}$ and $k=\omega_{r} R_{s} / 2 Q$. After a Fourier transform, this wakefield becomes a narrow-band impedance peaked at $\omega_{r}$ and given by

$$
Z_{\|}(\omega)=\frac{R_{s}}{1+i Q\left(\frac{\omega_{r}}{\omega}-\frac{\omega}{\omega_{r}}\right)} .
$$

This can be used as a simple model to represent a longitudinal cavity HOM.

\subsection{HIGHER ORDER MODES}

In Chapter 3, we discussed how the cavity geometry, which is defined by its conducting boundaries, only permits a discrete set of stationary electromagnetic modes to exist in the cavity: the eigenmodes of the cavity.

Radio-frequency cavities are designed to operate in a very specific mode, usually the lowest-frequency mode of the cavity, sometimes called the fundamental mode. We therefore call high-order modes (HOMs) to all the remaining electromagnetic modes in the cavity, which have higher frequency than the fundamental mode. In general, however, there could be a particular cavity where we would need to distinguish between lower, similar and higher order modes. HOMs are characterized by their frequency $\omega$, the shunt impedance $R / Q$, and its corresponding quality factor $Q$.

One of the characteristics of the RFD crab cavity is that it does not have lower order modes and therefore the $\mathrm{TE}_{110}$ mode at $952.6 \mathrm{MHz}$ is the fundamental mode. High order modes are excited by a passing bunch charge. A bunch with length $\sigma_{z} \sim \sigma_{t} c$ can excite HOMs with frequencies up to $\omega \sim \sigma_{t}^{-1}$. The JLEIC high-luminosity plan involves using 
short bunches. A JLEIC type bunch with length $\sigma_{z}=1.2 \mathrm{~cm}$ has the potential to excite HOMs up to about $25 \mathrm{GHz}$.

A bunch traveling along the axis of the cavity will excite monopole modes, or longitudinal HOMs. If the bunch travels at an offset relative to the cavity axis, then it will excite transverse HOMs. It is very convenient to treat separately the longitudinal and transverse HOMs.

A cavity in a particle accelerator is not an ideal closed structure because of the beam pipes. Because of this, there can be both trapped and propagating modes inside the cavity. Trapped modes have frequencies below the pipe cut-off frequency and thus can not propagate out of the cavity, while propagating modes can travel outside the cavity into the beam pipe, because they have frequencies higher than the beam pipe cut-off frequency. The pipe cutoff frequency is determined by its diameter, and in turn matched to the cavity aperture diameter.

For the coupled-bunch instability studies in Section 6.3, we are only interested in trapped modes. Considering the beam aperture of crab cavities to be $70 \mathrm{~mm}$, the (cylindrical) beam pipe cut-off frequency is given by

$$
f_{\mathrm{c}}=\frac{c}{\pi D} X
$$

with $X$ the root of the Bessel function and $D$ the beam pipe aperture. This correspond to 2.51 GHz for $\mathrm{TE}_{11}$ and $3.278 \mathrm{GHz}$ for $\mathrm{TM}_{01}$ modes. HOMs up to this frequencies need to be included in the CBI calculation.

\subsubsection{CRAB CAVITY FOR JLEIC}

Two different cavity designs are used for the study of HOMs: a 2-cell and 3-cell 952.6 $\mathrm{MHz}$ RFD crab cavity with $70 \mathrm{~mm}$ beam pipe aperture. Both were modeled using CST Microwave Studio and are shown in Fig. 59. Hooks and probes were added for this study, but further optimization are part of the on-going crab cavity R\&D. Figure 60 shows the impedance of longitudinal HOMs up to $3 \mathrm{GHz}$ for both cavity designs. 

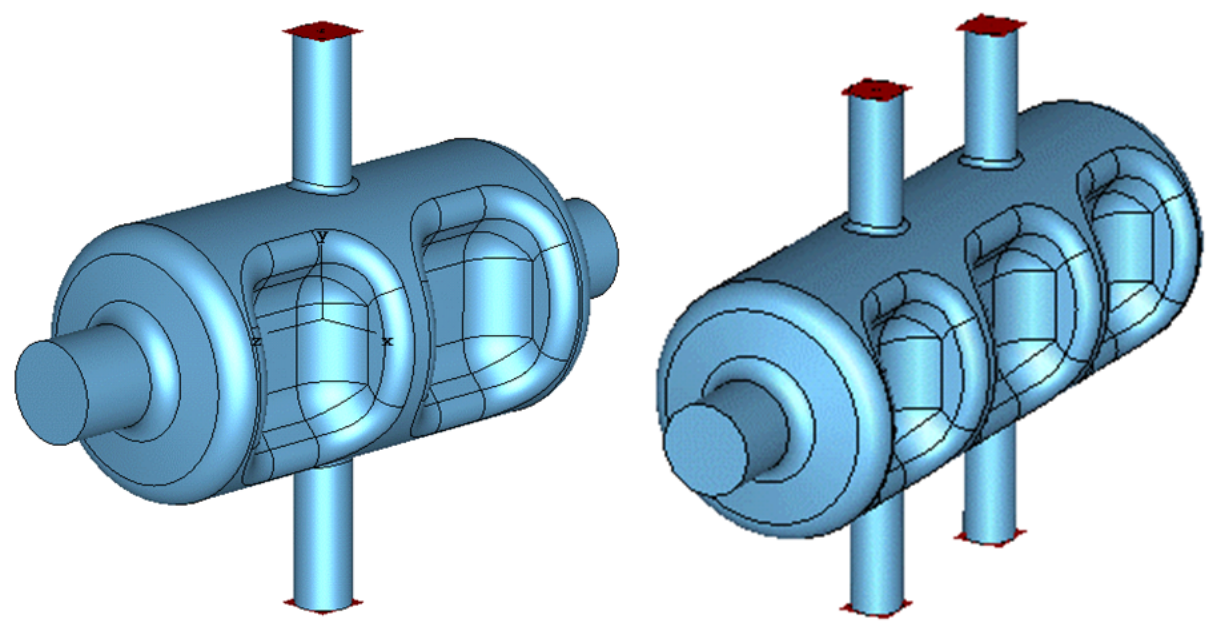

FIG. 59: Crab cavity concepts with hooks and probes for study of HOMs: 2-cell and 3-cell 952.6 $\mathrm{MHz}$ cavities with $70 \mathrm{~mm}$ aperture.

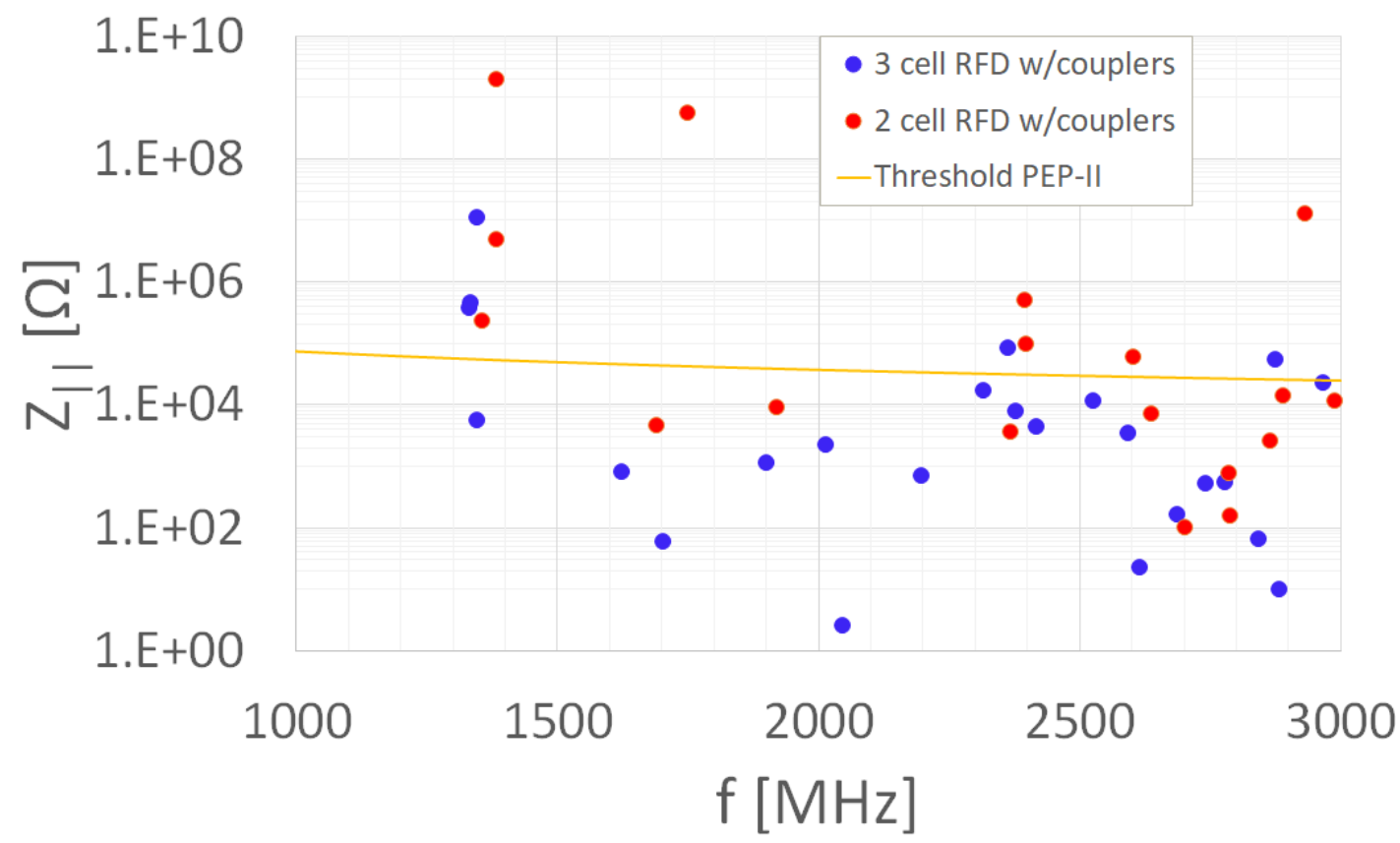

FIG. 60: Longitudinal HOM impedance from 2 and 3-cell crab cavity rf-dipole designs. The solid line shows the threshold impedance assuming the state-of-art rf feedback system used in PEP-II. 


\subsection{COUPLED BUNCH INSTABILITY}

A bunch traveling through the ring can excite wakefields along different machine elements. RF cavities in particular are designed to be highly-resonant structures. The induced wakefields may not be completely damped by the time a new bunch comes into the cavity, as illustrated in Fig. 61. If this is the case, then bunches can become coupled to each other

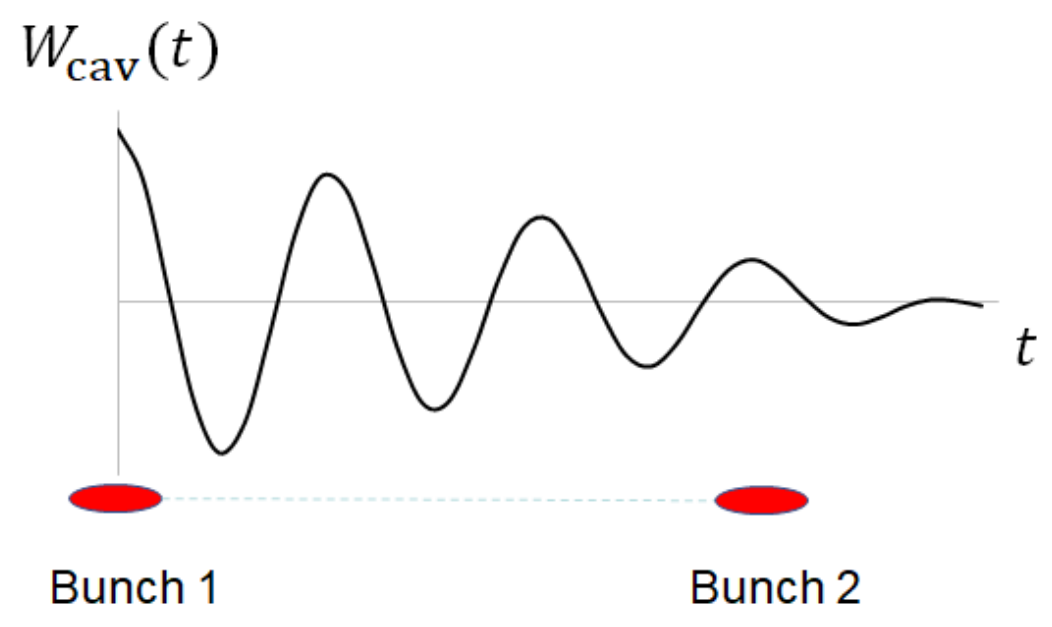

FIG. 61: Bunches in a beam can become coupled by a long-ringing wakefield in an rf cavity.

and may start to oscillate coherently. Physically, the dynamics closely relates to a damped system of coupled oscillators with a driving external force.

Assuming the beam is formed by $M$ equally spaced bunches, there are $M$ different coupled-bunch modes (CBM) of oscillation. The CBM are characterized by an index $\mu$, $0 \leq \mu<M$, where $2 \pi \mu / M$ is the phase shift between the oscillation of adjacent bunches. A second index $a$ describes the oscillating mode of a single bunch in the synchrotron phase space, with $a=1$ the dipole mode of oscillation, where the bunch oscillates rigidly; $a=2$ is the quadrupole mode, with head and tail of the bunch oscillating out of phase, etc.

If the amplitude of oscillation for a particular CBM steadily grows, it can give rise to a Coupled-Bunch Instability (CBI). Trapped modes in the crab cavity can drive CBI since they coherently effect subsequent passing bunches. CBI have a characteristic rise, or growth time $\tau$. A stability criterion for the CBI is for its growth time $\tau_{\text {growth }}^{i}$ to be longer than the damping time $\tau_{\text {damp }}$ :

$$
\tau_{\text {growth }}^{i}>\tau_{\text {damp }},
$$

where the damping can be provided through various mechanisms like synchrotron radiation, 
Landau damping, or bunch by bunch feedback kicker.

Another option is to engineer the cavity HOMs by lowering the quality factor of any particularly disruptive HOMs driving a CBI. With sufficiently damped HOMs, conventional RF feedback systems can be used to handle the instability growth. Figure 62 shows a calculation scheme to identify troublesome HOMs: those coupling stronger to the beam current spectrum.

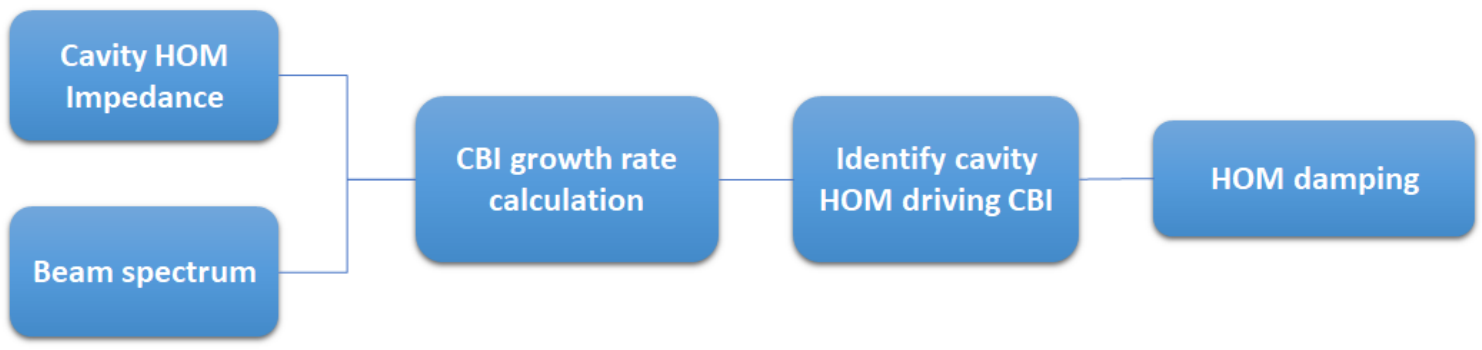

FIG. 62: Diagram of the calculation to identify cavity HOMs driving coupled-bunch oscillations.

\subsubsection{BEAM SPECTRUM}

An ideal case of a bunched beam is that of $M$ equally spaced point charges with electric charge $q_{\mathrm{b}}$. This bunched beam can be modeled as a train of equally spaced delta functions. The beam current at a particular location on the ring is then

$$
I(t)=q \sum_{k=-\infty}^{\infty} \delta\left(t-k \frac{T_{0}}{N}\right)=\frac{N q}{T_{0}} \sum_{k=-\infty}^{\infty} e^{i k N \omega_{0} t},
$$

where $T_{0}=2 \pi R / \beta c$ is the period of revolution. The frequency component of the current is obtained through a Fourier transform

$$
I(\omega)=\int_{-\infty}^{\infty} I(t) e^{-i \omega t} d t=\frac{q N \omega_{0}}{2 \pi} \sum_{k=-\infty}^{\infty} \delta\left(\omega-k N \omega_{0}\right)
$$

The uniform beam spectrum has the sampling frequencies $\omega_{p, \mu, a}=(p M+\mu) \omega_{0}+a \omega_{s}$, where $p$ is an integer, $\omega_{0}$ is the beam revolution frequency, $\omega_{s}$ is the synchrotron frequency and $a$ gives the synchrotron mode of oscillation. Furthermore, if one considers the bunch distribution of particles, then the Fourier transform of the train of bunches becomes an uniformly spaced train of frequencies $\omega_{p, \mu, a}$ with an envelope defined by the single bunch distribution, as shown in Fig. 63. For a Gaussian bunch, the envelope is

$$
\frac{I(\omega)}{q}=\exp \left[-\frac{1}{2}\left(\frac{\omega}{c} \sigma_{r m s}\right)^{2}\right]
$$


while for a parabolic bunch, the distribution is

$$
\frac{I(\omega)}{q}=\frac{1}{2}\left[1+\cos \left(\frac{\omega}{c} \sigma_{r m s}\right)\right]
$$

Figure 63 shows the uniform beam spectrum assuming a $\sigma_{z}=1.2 \mathrm{~cm}$ bunch length and both parabolic and Gaussian distributions.

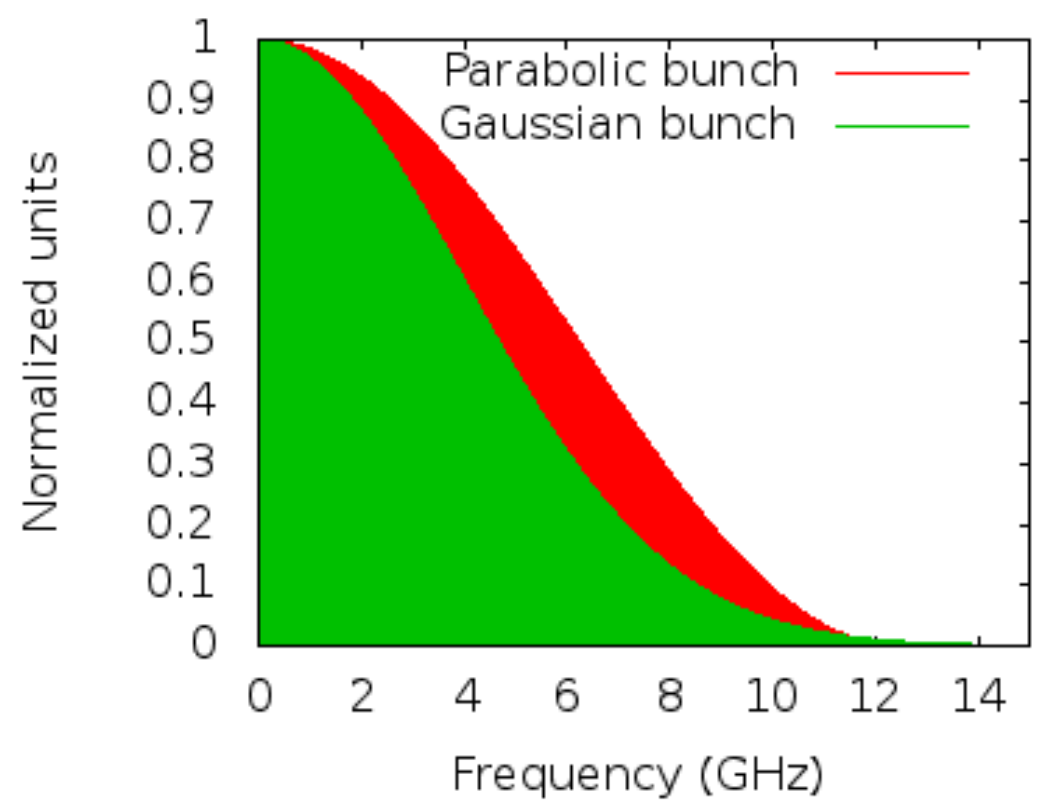

FIG. 63: Beam spectrum of an uniformly-spaced train of bunches with Gaussian and parabolic distributions.

A couple of comments are necessary at this point. First, note the reason high-frequency HOMs do not need to be considered in the CBI calculation is that the beam current spectrum, defined by the bunch length, does not have Fourier components at high frequency, i.e., they can not couple. Second, a uniformly-filled beam spectrum model generally gives an overestimation on the calculations. In reality the beam can have a more interesting structure. For example, the bunch train must have gaps, which are sufficiently long enough for the injection/extraction kicker to turn on, abort gaps, etc. 


\subsubsection{EFFECTIVE IMPEDANCE}

We are mostly concerned about unstable modes of oscillation that give rise to a CBI. A very detailed study of CBI using the oscillations of bunch centroids is presented in [56]. The $\mathrm{CBI}$ is characterized by its growth time, and can be calculated through [35]:

$$
\begin{aligned}
\tau_{\|}^{-1} & =C_{0} \sum_{p} \frac{R e\left[Z_{\|}\left(\omega_{p}\right)\right]}{\left(\omega_{p} / \omega_{0}\right)} \frac{h_{a}\left(\omega_{p}\right)}{S_{a}}, \\
C_{0} & =\frac{I_{b} \omega_{0}^{2} \eta}{6(L / 2 \pi R)^{3} 2 \pi \beta^{2}(E / e) \omega_{s}}
\end{aligned}
$$

this is a weighted bunch power spectra sampled at the beam frequencies. For our calculations, we assume a parabolic bunch power spectra $h_{a}$

$$
h_{a}(\omega)=(a+1)^{2} \frac{1+(-1)^{a} \cos \left(\omega / \omega_{b}\right)}{\left[\left(\omega / \pi \omega_{b}\right)^{2}-(a+1)^{2}\right]^{2}},
$$

$\omega_{b}=\beta c / 2 \sqrt{2} \sigma_{z}$. The Gaussian bunch power spectrum is

$$
h_{a}(\omega)=\left(\frac{\omega}{\omega_{b}}\right)^{2 a} \exp \left[-\left(\frac{\omega}{\omega_{b}}\right)^{2}\right],
$$

$\omega_{b}=\beta c / \sigma_{z}$. The ring parameters used are consistent with CBI calculations for JLEIC accelerating rf cavities as presented in [57], presented in Table 9.

TABLE 9: Parameters used in CBI calculation.

\begin{tabular}{|l|c|}
\hline Parameter & Ion ring \\
\hline Circumference & 2153.9 \\
\hline Energy spread & $2 \times 10^{-4}$ \\
\hline No. of cavities & 14 \\
\hline Bunch length & 1.2 \\
\hline Bunch current & $0.218 \mathrm{~mA}$ \\
\hline Synchrotron tune & 0.0542 \\
\hline Ref. Frequency & $0.139 \mathrm{MHz}$ \\
\hline
\end{tabular}

Various mechanisms can provide some level of damping, for example, synchrotron radiation or bunch by bunch rf feedback system. In the case of the ion beam, however, synchrotron radiation damping time is very long and an rf feedback system is required. Preliminary impedance estimations suggest that the required kickers to damp instabilities in the ion ring will significantly increase the broadband impedance. The use of kickers is therefore not desirable and the crab cavity is being designed to rely on HOM dampers to absorb any beam-damaging HOM. 
The state-of-art feedback system used at PEP-II can handle growth times on the order of milliseconds and can be used to set an impedance threshold on the crab cavity HOM impedance. The longitudinal threshold impedance is

$$
Z_{\|}(f)=\frac{2 \nu_{s} E_{\mathrm{b}}}{N_{\text {cavi }_{\mathrm{b}}} \alpha \tau} \frac{1}{f} .
$$

The transverse threshold impedance is

$$
Z_{x, y}(f)=\frac{2 E_{\mathrm{b}}}{N_{\mathrm{cav}} f_{\mathrm{rev}} I_{\mathrm{b}} \beta_{x, y} \tau_{x, y}}
$$

Parameters used to determine the threshold impedance are given in Table 10.

TABLE 10: Parameters used to estimate the threshold impedance assuming the state-of-art PEP-II feedback system.

\begin{tabular}{|l|c|}
\hline Parameter & Ion ring \\
\hline Beam energy & $100 \mathrm{GeV}$ \\
\hline Rev. Frequency & $0.139 \mathrm{MHz}$ \\
\hline Synch. tune & 0.0542 \\
\hline Mom. compaction factor & $6.41 \times 10^{-3}$ \\
\hline$\beta_{x}$ & $363.44 \mathrm{~m}$ \\
\hline$\beta_{y}$ & $1 \mathrm{~m}$ \\
\hline Beam current & $0.75 \mathrm{~A}$ \\
\hline No. of Cavities & 14 \\
\hline
\end{tabular}

Figure 60 includes the longitudinal threshold impedance assuming the state-of-art damping time provided by the PEP-II bunch by bunch feedback system. This shows that further optimization on the HOM absorbers is needed to reduce the HOM impedance of the cavity.

\subsubsection{CALCULATIONS}

We use a simple model for the longitudinal impedance of a cavity

$$
Z_{\|}(\omega)=\sum_{k} \frac{R_{s, k}}{1+i Q_{k}\left(\frac{\omega_{k}}{\omega}-\frac{\omega}{\omega_{k}}\right)},
$$

where the index $k$ runs over all possible HOMs. ZAP [58] allows for an uniform fill estimation, which is actually an overestimation of the realistic case. ZAP can also calculate different instability modes (synchrotron modes for longitudinal and betatron modes for transverse coupled bunch instabilities).

We calculate the longitudinal CBI growth rate for all the possible beam frequencies up to $3 \mathrm{GHz}$ using JLEIC ion ring parameters [57]. A factor of 0.1 is assumed to lower the $Q$ 
of HOMs to simulate a frequency spread due to cavity to cavity differences, as implemented in ZAP [58]. Figure 64 shows the CBI growth rate as a function of the CBM number $\mu$. Negative growth rates represented damped or stable modes of oscillation.

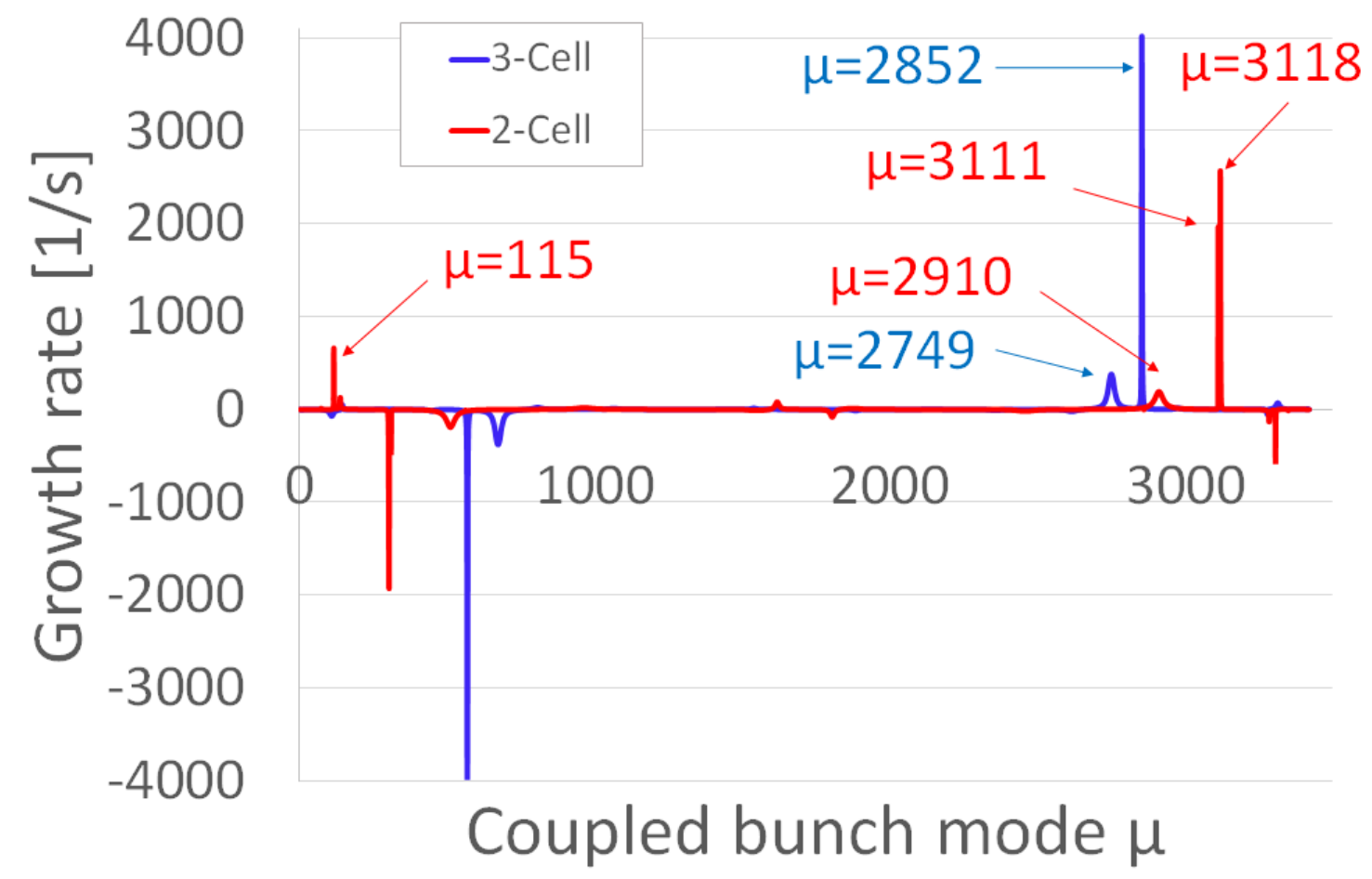

FIG. 64: Coupled-bunch instability growth rate vs. mode number corresponding to the 2 sets of crab cavity longitudinal HOMs.

For the 2-cell cavity, the CBM with $\mu=3118$ has the fastest CBI growth time of 0.39 ms. Similarly, for the 3-cell cavity, the fastest growth time is $0.25 \mathrm{~ms}$ for CBM $\mu=2852$. These modes can not be handled by a PEP-II type rf feedback system, and thus need to be addressed by optimizing the HOM couplers on the cavity. With the knowledge of the growth rate and CBM number, we can identify which HOM drives the instability by evaluating the beam frequency at the corresponding $\mu$ and scanning over the integers $p$ to find the closest beam frequency to a cavity HOM. Table 11 shows these beam frequencies and coupling HOM for the two cavity designs. 
TABLE 11: Beam spectrum frequencies matched to crab cavity HOMs. Top (bottom) values correspond to the 2(3)-cell cavity.

\begin{tabular}{|c|c|c|c|c|}
\hline Mode & $\mu$ & $\mathbf{p}$ & Beam $_{\mu, \mathbf{p}}[\mathrm{MHz}]$ & HOM f $[\mathbf{M H z}]$ \\
\hline 1 & 3118 & 2 & 1384.17 & 1385 \\
\hline 2 & 3111 & 2 & 1383.2 & 1385 \\
\hline 3 & 115 & 6 & 2868.27 & 2866 \\
\hline 4 & 2910 & 2 & 1355.26 & 1357 \\
\hline \hline 1 & 2852 & 2 & 1347.2 & 1349 \\
\hline 2 & 2749 & 2 & 1332.89 & 1334 \\
\hline 3 & 2749 & 5 & 2759.02 & 2743 \\
\hline
\end{tabular}

\subsection{HOM POWER}

Besides the instability considerations arising from HOMs, the HOM power induced by the beam needs to be taken into account when designing the refrigeration load required on the cryogenic module of the crab cavity. If HOMs in the cavity are not carefully engineered, the amount of power deposited on the cryogen can become excessive. Here we present an estimation assuming the worst possible scenario: where a beam spectrum line falls exactly on resonance with an HOM peak. In this case, the HOM power can be estimated [59] using

$$
P_{H O M}=I_{\mathrm{b}}^{2} \frac{R}{Q} Q_{\mathrm{L}} F_{n}^{2},
$$

where $I_{\mathrm{b}}$ is the average beam current, $(R / Q) Q_{\mathrm{L}}$ the impedance of the HOM and $F_{n}$ is the bunch form factor, extracted from the bunch power distribution shown in Fig. 63. The estimated HOM power under this assumptions is shown in Fig. 65, where we have assumed a $Q_{\mathrm{L}}=10^{6}$ for both crab cavities.

In a more realistic scenario, the HOM power dissipated can be calculated with [59]

$$
P(\omega)=\sum_{k=0}^{\infty} I(\omega)^{2} \cdot \operatorname{Re}[Z(\omega)],
$$

where a more accurate HOM impedance model can be calculated for a given structure. Similarly for the beam current spectrum; beams have a more interesting structure than a uniform fill, for example, they should include gaps for injection and extraction. Bunches may have different charges, etc, making the HOM power calculation more interesting. An example of a HOM power calculation using a beam spectrum involving trains of bunches is presented in Ref. [60]. Estimations of beam instabilities due to RF systems are generally done using available software. 


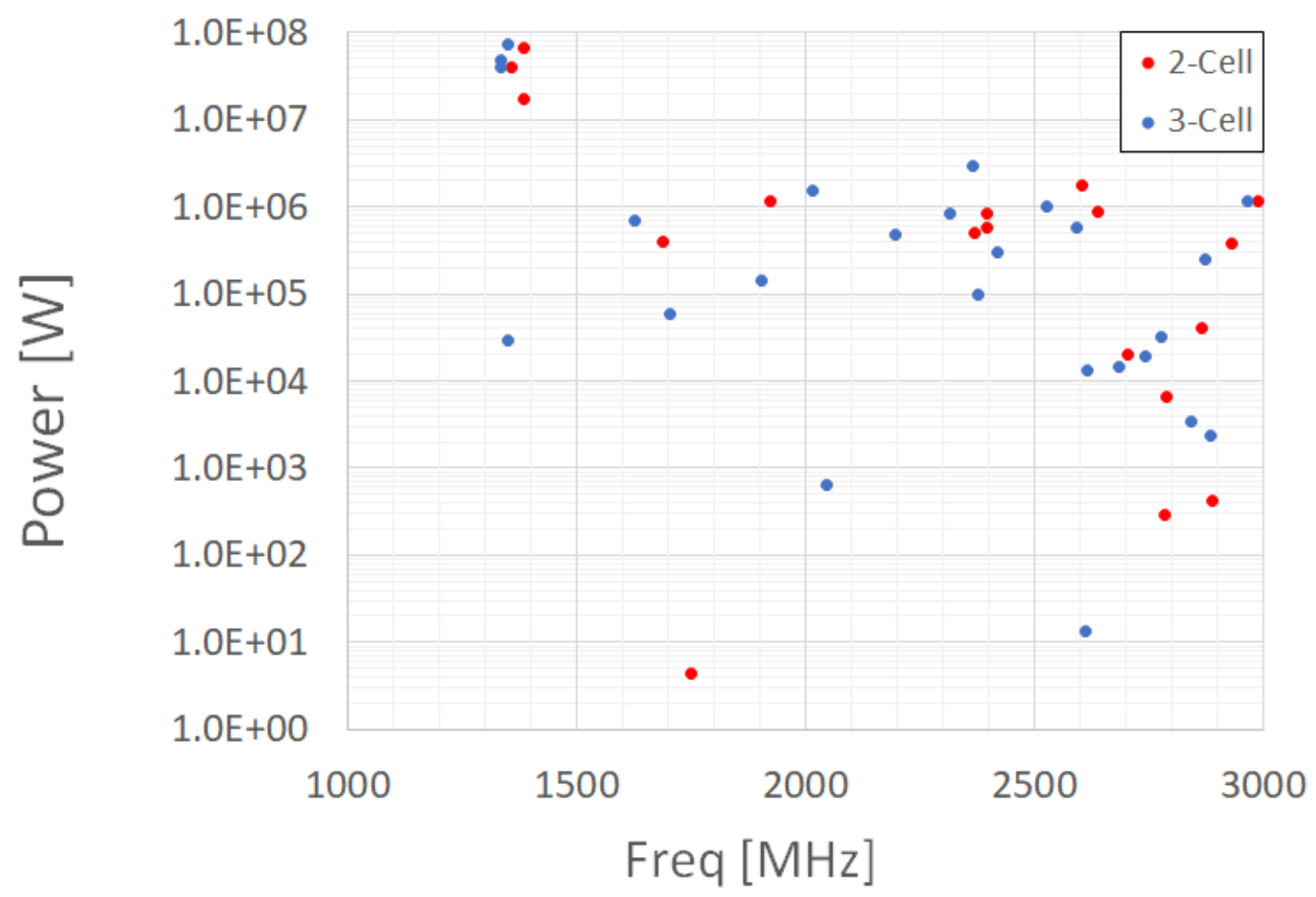

FIG. 65: Power from HOMs estimated for on-resonance excitation case.

A code developed at ANL called clinchor calculates the growth rates of longitudinal and transverse coupled bunch modes due to RF cavity HOMs. Clinchor code allows for a general beam current spectrum, not necessarily uniform, this is based on a normal mode analysis depeloped by K. Thompson [61]. This is helpful on providing an instability estimation based on a realistic beam filling scheme. Clinchor only estimates bunch centroid motion mode. Clinchor has been used in the past to study APS multi-cell RF cavities in deflecting modes [62, 63]. 


\section{CHAPTER 7}

\section{CONCLUSIONS}

One of the goals driving the design of JLEIC is the requirement of high-luminosity collisions in the $10^{34} \mathrm{~cm}^{-2} \mathrm{~s}^{-1}$ range. Part of the JLEIC strategy towards high-luminosity involves the integration of a crab crossing scheme in the interaction region. This scheme compensates for the geometric luminosity loss due to a relatively large $50 \mathrm{mrad}$ beam crossing angle. This scheme requires the use of superconducting radio-frequency crab cavities. Different designs of a crab cavity for JLEIC are currently being investigated. Of these, a multi-cell RFD crab cavity seems an adequate cavity, as it can provide the required crabbing voltage while keeping a compact size. On the other hand, single-cell RFD crab cavities become prohibitive because of the large number of cavities needed for high-energy ion beams.

In this dissertation, we implemented a bunch crabbing scheme in the available models of JLEIC electron and ion rings. Particularly, in the baseline for the ion ring we achieved sufficiently stable beams to use this model for further studies of dynamic aperture and rf noise. We also presented calculations of the coupled-bunch instability (CBI) growth time using preliminary longitudinal HOM data corresponding to 2-cell and 3-cell RFD crab cavities and assuming a uniform bunch fill in the ring. The uniform beam fill generally overestimates the CBI growth time. The fastest CBI growth time, which is a fraction of a millisecond for both cavities, is faster than the state-of-art rf feedback system $(\sim 2.5 \mathrm{~ms})$. The HOM dampers need to be optimized to make the CBI growth time longer and to reduce the induced HOM power. This is work on progress by H. Park and S. de Silva at CAS-ODU.

\subsection{CRAB CAVITY REQUIREMENTS}

Based on the JLEIC ring configuration presented in Chapter 4, the particle tracking studies in Chapter 5, and the CBI calculations in Chapter 6, we summarize the following requirements on a crab cavity for JLEIC:

- The rings of JLEIC are designed with chromaticity compensation blocks (CCB). These optical sections have high $\beta_{\mathbf{x}}$ values and betatron phase advance consistent with a local crabbing scheme. 
- The cavity aperture must have at least $70 \mathrm{~mm}$ diameter. Although a smaller aperture would give better rf performance, the cavity must be compatible with the ring during beam injection, when the beam has the largest size. A $70 \mathrm{~mm}$ cavity aperture guarantees enough space for the beam in injection mode.

- Cavity multipoles must not exceed (individually) the tolerances presented in Table 8. These values are based on the effect of multipoles on the dynamic aperture (DA) of the ring. All cavities considered yield acceptable dynamic apertures and are not a limitation to the DA.

- The RF voltage noise needs to be controlled at the $0.01 \%$ level for negligible impact on beam emittance. The $\mathbf{R F}$ phase noise needs to be kept below the 1 mdeg level for beam stability. This seems to be a limiting issue. A more relaxed 10 mdeg level would be desirable.

- Further optimization of crab cavity HOM absorbers is required for longer CBI growth times and reduced HOM power, even in the assumed worst case of HOM resonance excitation.

\subsection{FUTURE WORK}

We now discuss the impressions on work that needs to be completed next and recent changes that have been discussed on the ion ring relevant to the crab crossing scheme.

\subsubsection{OPTIMIZED VERSION OF JLEIC RINGS}

It is still necessary to perform bunch crabbing simulations with updated versions of the JLEIC electron and ion rings that are also optimized for crab cavities. A newer version of the ion ring has already reserved $10 \mathrm{~m}$ of space for crabbing systems but is not yet available for simulations. Once the cavity space is reserved in the ring, it would be possible to use the field map of a cavity instead of a linear model. This will give more precise results on the effect of multipoles in the ring.

Similarly, it has recently been discussed [45] that the optics of the ion ring can be adjusted to have a higher beta value, $\beta_{x}=450 \mathrm{~m}$ at the crab cavity locations, thus reducing the crab cavity voltage by a few MV. Crabbing parameters with this update are shown in Table 12 .

\subsubsection{REVISITED FREQUENCY OF THE CRAB CAVITY}


TABLE 12: Crabbing parameters of the ion ring with a higher betatron function value $\beta_{x}=450 \mathrm{~m}$ at the locations of crab cavities.

\begin{tabular}{|c|c|c|c|c|}
\hline Parameter & \multicolumn{3}{|c|}{ Ion ring } & Units \\
\hline Energy & 20 & 60 & 100 & $\mathrm{GeV}$ \\
\hline Frequency & \multicolumn{3}{|c|}{952.6} & $\mathrm{MHz}$ \\
\hline Crossing angle & \multicolumn{3}{|c|}{50} & mrad \\
\hline$\beta_{x}^{*}$ & \multicolumn{3}{|c|}{0.1} & $\mathrm{~m}$ \\
\hline$\beta_{x}^{c r a b}$ & \multicolumn{3}{|c|}{450} & $\mathrm{~m}$ \\
\hline Crab voltage & 3.74 & 11.2 & 18.7 & MV \\
\hline
\end{tabular}

It has also been discussed that a $476.3 \mathrm{MHz}$ crab cavity involves less technological risk, therefore making a stronger case for the JLEIC design before the EIC selection in 2020. For a $476.3 \mathrm{MHz}$ cavity, updated crabbing parameters of both electron and ion rings are presented in Table 13. Although the required crabbing voltage seems to be higher for the TABLE 13: Crabbing parameters of the electron and ion rings with $476.3 \mathrm{MHz}$ crab cavities.

\begin{tabular}{|l|c|c|c|c|c|c|}
\hline Parameter & Electron ring & \multicolumn{3}{|c|}{ Ion ring } & Units \\
\hline Energy & 5 & 10 & 20 & 60 & 100 & $\mathrm{GeV}$ \\
\hline Frequency & \multicolumn{5}{|c|}{$\mathbf{4 7 6 . 3}$} & $\mathrm{MHz}$ \\
\hline Crossing angle & \multicolumn{5}{|c|}{00} & $\mathrm{mrad}$ \\
\hline$\beta_{x}^{*}$ & \multicolumn{5}{|c|}{0.1} & $\mathrm{~m}$ \\
\hline$\beta_{x}^{\text {crab }}$ & \multicolumn{5}{|c|}{200} & \multicolumn{4}{|c|}{} & $\mathrm{m}$ \\
\hline Crab voltage & $\mathbf{2 . 8}$ & $\mathbf{5 . 6}$ & $\mathbf{7 . 4 7}$ & $\mathbf{2 2 . 4}$ & $\mathbf{3 7 . 3 5}$ & $\mathrm{MV}$ \\
\hline
\end{tabular}

ion ring, preliminary studies using CST Microwave Studio suggest a $476.3 \mathrm{MHz}$ cavity has a larger field gradient, yet the number of cavities needed to produce the crabbing voltage in the ion ring is similar as the $952.6 \mathrm{MHz}$ cavity: $\sim 10-12$ per beam per side of the interaction region. Figure 66 shows a comparison of the crabbed bunch at IP using a $476.3 \mathrm{MHz}$ element in the ion ring over one turn. 


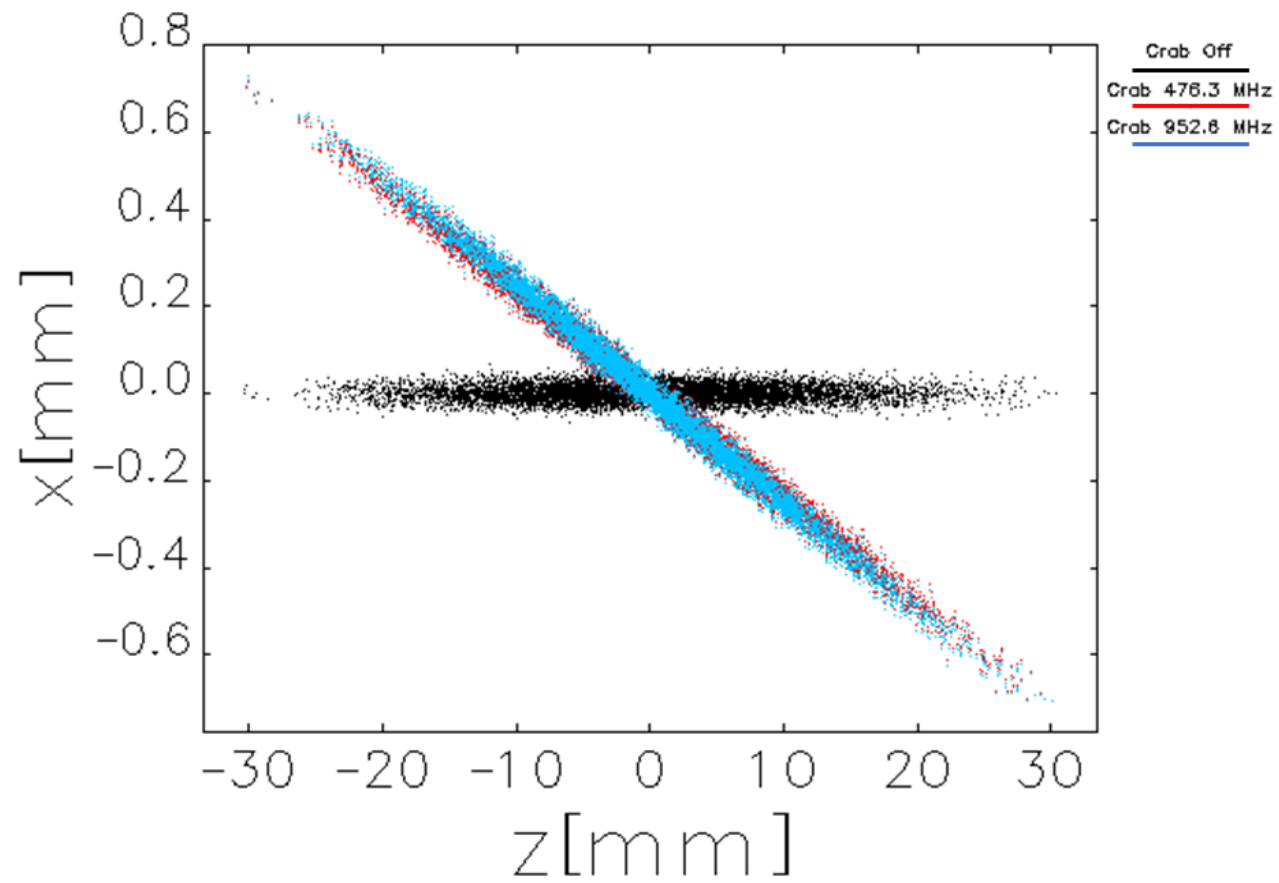

FIG. 66: Crabbed bunch at IP using 476.3 MHz and 952.6 MHz crabbing parameters.

\subsubsection{HOM STUDIES}

Similarly, calculations using the HOM of crab cavities where the HOM absorbers have been optimized need to be completed, this would include calculation of transverse CBI. In this work, our calculations were based on a simple HOM model. Using an optimized cavity, it is possible to numerically calculate the impedance of the actual cavity given a beam passing through it. This type of calculation can be done using CST Microwave Studio. A more accurate model of CBI calculation will also include a more realistic beam structure that includes gaps, or the actual JLEIC beam structure when the beam formation scheme is completed. With a more mature model of the JLEIC rings, it will also be possible to implement consistent particle tracking studies including crab cavity HOM impedance. 


\section{BIBLIOGRAPHY}

[1] Y. Zhang, "JLEIC Baseline Update," (2018), spring 2018 JLEIC Collaboration Meeting, Jefferson Lab.

[2] Y. Funakoshi (for the KEKB Commissioning Group), "Operational experience with crab cavities at KEKB,", 27-36. 10 p (2014).

[3] Frank Zimmermann and Ulrich Dorna, Progress of Beam-Beam Compensation Schemes, Tech. Rep. (CERN, 2005).

[4] "World's first crabbing of a proton beam," (2018), Accelerating News, Issue 25.

[5] Microwave Studio, CST, Tech. Rep. (GmbH, Darmstadt, Germany, 2000).

[6] F. Lin et al., "JLEIC Collider Rings' design," (2018), spring 2018 JLEIC Collaboration Meeting, Jefferson Lab.

[7] "2007 NSAC Long Range Plan: The Frontiers of Nuclear Science," https://science. energy.gov/ /media/np/nsac/pdf/docs/NuclearScienceHighRes.pdf (2007).

[8] "2015 NSAC Long Range Plan: Reaching for the Horizon," https://science.energy . gov/ /media/np/nsac/pdf/2015LRP/2015_LRPNS_091815.pdf (2015).

[9] A. Accardi, J. L. Albacete, M. Anselmino, N. Armesto, E. C. Aschenauer, A. Bacchetta, D. Boer, W. K. Brooks, T. Burton, N. B. Chang, W. T. Deng, A. Deshpande, M. Diehl, A. Dumitru, R. Dupré, R. Ent, S. Fazio, H. Gao, V. Guzey, H. Hakobyan, Y. Hao, D. Hasch, R. Holt, T. Horn, M. Huang, A. Hutton, C. Hyde, J. JalilianMarian, S. Klein, B. Kopeliovich, Y. Kovchegov, K. Kumar, K. Kumerički, M. A. C. Lamont, T. Lappi, J. H. Lee, Y. Lee, E. M. Levin, F. L. Lin, V. Litvinenko, T. W. Ludlam, C. Marquet, Z. E. Meziani, R. McKeown, A. Metz, R. Milner, V. S. Morozov, A. H. Mueller, B. Müller, D. Müller, P. Nadel-Turonski, H. Paukkunen, A. Prokudin, V. Ptitsyn, X. Qian, J. W. Qiu, M. Ramsey-Musolf, T. Roser, F. Sabatié, R. Sassot, G. Schnell, P. Schweitzer, E. Sichtermann, M. Stratmann, M. Strikman, M. Sullivan, S. Taneja, T. Toll, D. Trbojevic, T. Ullrich, R. Venugopalan, S. Vigdor, W. Vogelsang, C. Weiss, B. W. Xiao, F. Yuan, Y. H. Zhang, and L. Zheng, "Electron-Ion Collider: The next QCD frontier," The European Physical Journal A 52, 268 (2016). 
[10] M Farkhondeh and V Ptitsyn, eRHIC Zeroth-Order Design Report, Tech. Rep. (Brookhaven National Laboratory, NY (USA), 2004).

[11] S Abeyratne, D Barber, A Bogacz, P Brindza, Y Cai, A Camsonne, A Castilla, P Chevtsov, E Daly, YS Derbenev, Krafft G. A. (editor), et al., "MEIC design summary," arXiv preprint arXiv:1504.07961 (2015).

[12] R. B. Palmer, "Energy scaling, crab crossing, and the pair problem," eConf 8806271, 613-619 (1988).

[13] Katsunobu Oide and Kaoru Yokoya, "Beam-beam collision scheme for storage-ring colliders," Physical Review A 40, 315 (1989).

[14] Apollinari G., Béjar Alonso I., Brüning O., Fessia P., Lamont M., Rossi L., and Tavian L., High-Luminosity Large Hadron Collider (HL-LHC): Technical Design Report V. 0.1, CERN Yellow Reports: Monographs (CERN, Geneva, 2017).

[15] Lia Merminga, David R. Douglas, and Geoffrey A. Krafft, "High-Current EnergyRecovering Electron Linacs," Annual Review of Nuclear and Particle Science 53, 387429 (2003), https://doi.org/10.1146/annurev.nucl.53.041002.110456.

[16] Vasiliy Morozov, Yaroslav Derbenev, Leigh Harwood, Andrew Hutton, Fanglei Lin, Fulvia Pilat, Yuhong Zhang, Yunhai Cai, Y. M. Nosochkov, Michael Sullivan, M.-H. Wang, Uli Wienands, James Gerity, Thomas Mann, Peter McIntyre, Nathaniel Pogue, and Akhdiyor Sattarov, "Status of the MEIC ion collider ring design," in 6th International Particle Accelerator Conference, IPAC 2015 (2015).

[17] Ya. S. Derbenev, V. S. Morozov, F. Lin, Y. Zhang, A M. Kondratenko, M A. Kondratenko, and Yu Filatov, "Polarization Preservation and Control in a Figure-8 Ring," International Journal of Modern Physics: Conference Series 40, 1660090 (2016).

[18] Christoph W. Leemann, David R. Douglas, and Geoffrey A. Krafft, "The Continuous Electron Beam Accelerator Facility: CEBAF at the Jefferson Laboratory," Annual Review of Nuclear and Particle Science 51, 413-450 (2001), https://doi.org/10.1146/annurev.nucl.51.101701.132327.

[19] Michael F. Spata, "12 GeV CEBAF Initial Operational Experience and Challenges," 10.18429/JACoW-IPAC2018-WEYGBD1. 
[20] E.W. Nissen and T. Satogata, "Revised Optics Design for the JLEIC Ion Booster," in Proc. 9th International Particle Accelerator Conference (IPAC'18), Vancouver, BC, Canada, April 29-May 4, 2018, International Particle Accelerator Conference No. 9 (JACoW Publishing, Geneva, Switzerland, 2018) pp. 3537-3539, https://doi.org/10.18429/JACoW-IPAC2018-THPAK126.

[21] J. D. Jackson, Classical Electrodynamics (Wiley, New York, 1999).

[22] Hasan Padamsee, RF Superconductivity for Accelerators (Wiley-VCH, Weinheim, 2008).

[23] J. Bardeen, L. N. Cooper, and J. R. Schrieffer, "Theory of Superconductivity," Phys. Rev. 108, 1175-1204 (1957).

[24] W. K. H. Panofsky and W. A. Wenzel, "Some considerations concerning the transverse deflection of charged particles in radio-frequency fields," Review of Scientific Instruments 27, 967-967 (1956).

[25] Binping Xiao, Luís Alberty, Sergey Belomestnykh, Ilan Ben-Zvi, Rama Calaga, Chris Cullen, Ofelia Capatina, Lee Hammons, Zenghai Li, C Marques, John Skaritka, Silvia Andrés, and Qiong Wu, "Design, prototyping and testing of a compact superconducting double quarter wave crab cavity," Physical Review Special Topics - Accelerators and Beams 18 (2015), 10.1103/PhysRevSTAB.18.041004.

[26] S. U. De Silva and J. R. Delayen, "Design evolution and properties of superconducting parallel-bar rf-dipole deflecting and crabbing cavities," Phys. Rev. ST Accel. Beams 16, 012004 (2013).

[27] S. U. De Silva, A. Castilla, and J. R. Delayen, "Compact Superconducting RF-dipole Cavity Designs for Deflecting and Crabbing Applications," in Proceedings, 4th International Particle Accelerator Conference (IPAC 2013): Shanghai, China, May 12-17, 2013 (2013) p. WEPWO080.

[28] A. Castilla and J. R. Delayen, "Analysis of a $750 \mathrm{MHz}$ SRF Dipole Cavity," in Proceedings, 17th International Conference on RF Superconductivity (SRF2015): Whistler, Canada, September 13-18, 2015 (2015) p. THPB047.

[29] J. R. Delayen, A. Castilla, and G. A. Krafft, "Design of Electron and Ion Crabbing Cavities for an Electron-Ion Collider," in Proceedings, 3rd International Conference on 
Particle accelerator (IPAC 2012): New Orleans, USA, May 2-25, 2012, Vol. C1205201 (2012) pp. 2447-2449.

[30] S. U. De Silva and J. R. Delayen, "Cryogenic test of a proof-of-principle superconducting rf-dipole deflecting and crabbing cavity," Phys. Rev. ST Accel. Beams 16, 082001 (2013).

[31] A. Castilla, J. R. Delayen, and H. Park, "Cryogenic Test of a 750 MHz Superconducting RF Dipole Crabbing Cavity," (2014).

[32] S. U. De Silva, J. R. Delayen, and H. Park, "Multi-Cell RF-Dipole Deflecting and Crabbing Cavity," in Proceedings, 7th International Particle Accelerator Conference (IPAC 2016): Busan, Korea, May 8-13, 2016 (2016) p. WEPMW022.

[33] HyeKyoung Park, Alejandro Castilla, Subashini De Silva, Jean Delayen, and Vasiliy Morozov, "Analyses of $476 \mathrm{MHz}$ and $952 \mathrm{MHz}$ Crab Cavities for JLab Electron IonCollider," in 7th International Particle Accelerator Conference (IPAC'16), Busan, Korea, May 8-13, 2016 (JACOW, Geneva, Switzerland, 2016) pp. 2348-2350.

[34] Helmut Wiedemann, Particle Accelerator Physics (Springer, Berlin New York, 2007).

[35] Alexander Chao, Handbook of Accelerator Physics and Engineering (World Scientific Pub. Co, Singapore Hackensack, N.J, 2013).

[36] K M Potter, "Luminosity measurements and calculations; 1994 ed." (1994).

[37] M. Syphers, "Some Notes on Luminosity Calculations," (2007).

[38] Yong Ho Chin, "Effects of Non-Zero Dispersion at Crab Cavities on the Beam Dynamics," Lawrence Berkeley National Laboratory (2010).

[39] Yi-Peng Sun, Ralph Assmann, Javier Barranco, Rogelio Tomás, Thomas Weiler, Frank Zimmermann, Rama Calaga, Akio Morita, et al., "Beam dynamics aspects of crab cavities in the CERN Large Hadron Collider," Physical Review Special Topics-Accelerators and Beams 12, 101002 (2009).

[40] Shahid Ahmed, Geoffrey A. Krafft, Kirsten Deitrick, Subashini U. De Silva, Jean R. Delayen, Mike Spata, Michael Tiefenback, Alicia Hofler, and Kevin Beard, "Beam dynamics studies for transverse electromagnetic mode type rf deflectors," Phys. Rev. ST Accel. Beams 15, 022001 (2012). 
[41] Alejandro Castilla, Crabbing System for an Electron-Ion Collider, Ph.D. thesis, Old Dominion University (2017).

[42] Vasiliy S Morozov, Ya S Derbenev, Fanglei Lin, and Rolland P Johnson, "Symmetric achromatic low-beta collider interaction region design concept," Phys. Rev. ST Accel. Beams 16, 011004 (2013).

[43] Y. M. Nosochkov, Y. Cai, M. Sullivan, /SLAC, Ya.S. Derbenev, F. Lin, V.S. Morozov, F. Pilat, G.H. Wei, Y. Zhang, /Jefferson Lab., and M.-H. Wang, "Compensation of Chromaticity in the JLEIC Electron Collider Ring," (2017).

[44] Vasiliy Morozov, Yaroslav Derbenev, Fanglei Lin, Fulvia C. Pilat, Guohio Wei, Yuhong Zhang, Y. M. Nosochkov, Yunhai Cai, Michael K. Sullivan, and M. H. Wang, "Injection Optics for the JLEIC Ion Collider Ring," (2016).

[45] V. Morozov, private communication.

[46] S. U. De Silva, K. Deitrick, H. Park, and J. R. Delayen, "Beam dynamics studies of $499 \mathrm{MHz}$ superconducting rf-dipole deflecting cavity system," (2015).

[47] Alejandro Castilla, Vasiliy S Morozov, T Satogata, and Jean R Delayen, "Modeling Crabbing Dynamics in an Electron-Ion Collider," (2015).

[48] W. Herr and F. Schmidt, "A MAD-X primer," in Intermediate accelerator physics. Proceedings, CERN Accelerator School, Zeuthen, Germany, September 15-26, 2003 (2004) pp. 505-528.

[49] Michael Borland, Elegant: A flexible SDDS-compliant code for accelerator simulation, Tech. Rep. (Argonne National Lab., IL (USA), 2000).

[50] A Morita, Emittance growth due to crab cavity ramping for LHC beam-1 lattice, Tech. Rep. (CERN, 2008).

[51] J Barranco Garcia, R Calaga, R De Maria, M Giovannozzi, A Grudiev, and R Tomás, "Study of Multipolar RF Kicks from the main deflecting mode in compact crab cavities for LHC," in 3rd International Particle Accelerator Conference (2012) p. 1873.

[52] Payagalage Subashini Uddika De Silva and Jean Roger Delayen, "Multipole Field Effects for the Superconducting Parallel-Bar Deflecting/Crabbing Cavities," (2012). 
[53] Guohui Wei, Fanglei Lin, Vasiliy Morozov, Yuri Nosochkov, Fulvia Pilat, Min-Huey Wang, and Yuhong Zhang, "Influence of Magnet Multipole Field Components on Beam Dynamics in the JLEIC Ion Collider Ring," in 7th International Particle Accelerator Conference (IPAC'16), Busan, Korea, May 8-13, 2016 (JACOW, Geneva, Switzerland, 2016) pp. 3525-3527.

[54] R. A. Rimmer, J. M. Byrd, and D. Li, "Comparison of calculated, measured, and beam sampled impedances of a higher-order-mode-damped rf cavity," Phys. Rev. ST Accel. Beams 3, 102001 (2000).

[55] P. Baudrenghien and T. Mastoridis, "Fundamental cavity impedance and longitudinal coupled-bunch instabilities at the High Luminosity Large Hadron Collider," Phys. Rev. Accel. Beams 20, 011004 (2017).

[56] A Mosnier, "Cures of Coupled Bunch Instabilities," (1999).

[57] R. Li, "Estimations of Coherent Instabilities for JLEIC," (2017).

[58] M. Zisman et al., ZAP User's Manual, Tech. Rep. (LBL-21270 (USA), 1986).

[59] Rama Calaga and Benoit Salvant, "Comments on Crab Cavity HOM Power," (2013).

[60] Ivan Karpov, Rama Calaga, and Elena Shaposhnikova, "High order mode power loss evaluation in future circular electron-positron collider cavities," Phys. Rev. Accel. Beams 21, 071001 (2018).

[61] K. A. Thompson and R. D. Ruth, "Transverse coupled-bunch instabilities in damping rings of high-energy linear colliders," Phys. Rev. D 43, 3049-3062 (1991).

[62] L. Emery, T. Berenc, M. Borland, and R. Lindberg, "Multi-Bunch Stability Analysis of the Advanced Photon Source Upgrade Including the Higher-Harmonic Cavity," in Proceedings, 6th International Particle Accelerator Conference (IPAC 2015): Richmond, Virginia, USA, May 3-8, 2015 (2015) p. TUPJE065.

[63] L. Emery, "Required cavity HOM deQing calculated from probability estimates of coupled bunch instabilities in the APS ring," in Proceedings of the 1993 Particle Accelerator Conference (PAC 93): May 17-20, 1993 Washington D.C., Vol. C930517 (1993) pp. 3360-3362. 


\section{APPENDIX A}

\section{CODE FILES}

Code files used in this dissertation for particle tracking in elegand and for CBI calculations in python. Section A.1 is an elegant file used to define a proton bunch at $100 \mathrm{GeV}$, with the crabbing voltage ramped over 1000 turns. The resulting bunch phase space of the crabbed bunch is saved into a file. Section A.2 is a file used to track a crabbed bunch in the JLEIC ion ring baseline. The input bunch in this file is the output bunch of the code in Section A.1. The file in Section A.3 is used to calculate the dynamic aperture of the ion ring with crab cavities that have multipoles. Finally, the code in Section A.4 is used to calculate the CBI growth time corresponding to all the coupled-bunch modes of oscillation. Input is a text file with HOM data columns. The output is a file containing CBM mode number $\mu$ vs growth time $\tau$.

\section{A.1 ELEGANT FILE TO GENERATE A CRABBED BUNCH.}

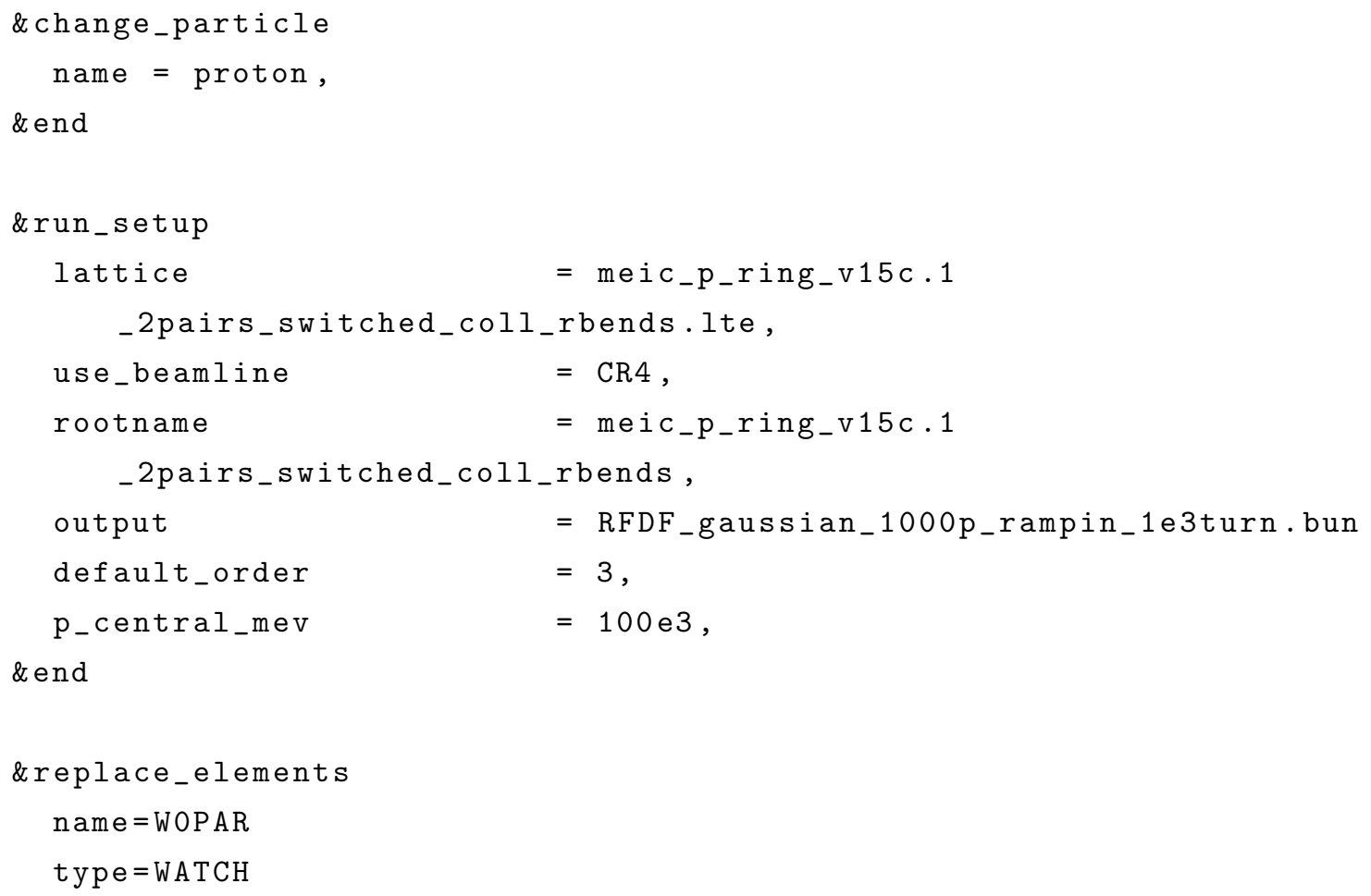


element_def = "rWOPAR: MARK"

\&end

\&replace_elements

name $=$ WIP

type $=$ WATCH

element_def $=$ "rWIP : MARK"

\&end

\&ramp_elements

item $=$ VOLTAGE

type $=$ RFDF

start_pass $=0$,

end_pass $=1000$,

start_value $=0$,

end_value $=20813739.2$,

verbose $=0$,

record $=r a m p . s d d s$,

\&end

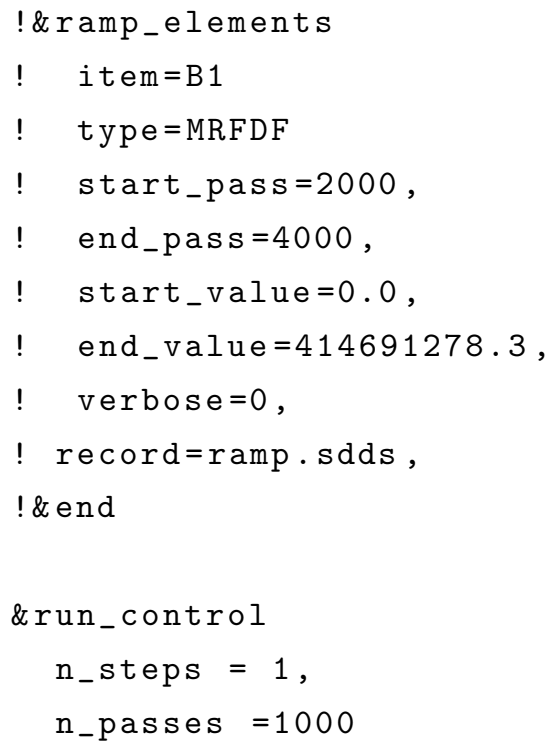




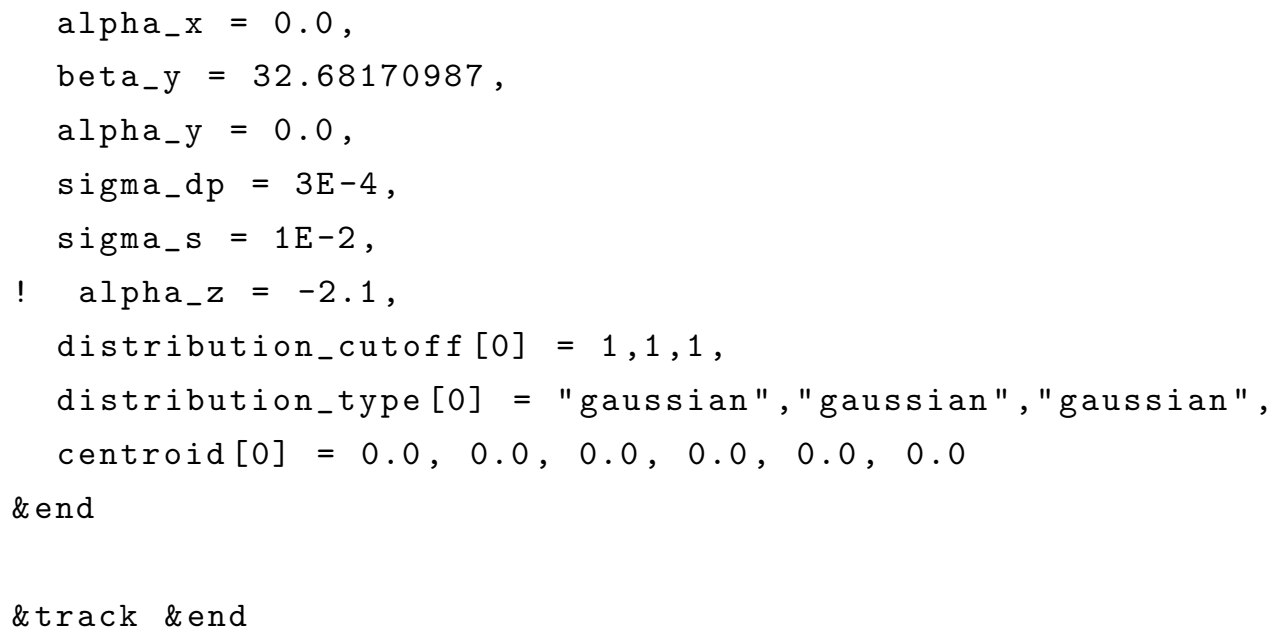

\section{A.2 ELEGANT CONFIGURATION FILE TO TRACK A CRABBED BUNCH IN THE ION RING.}

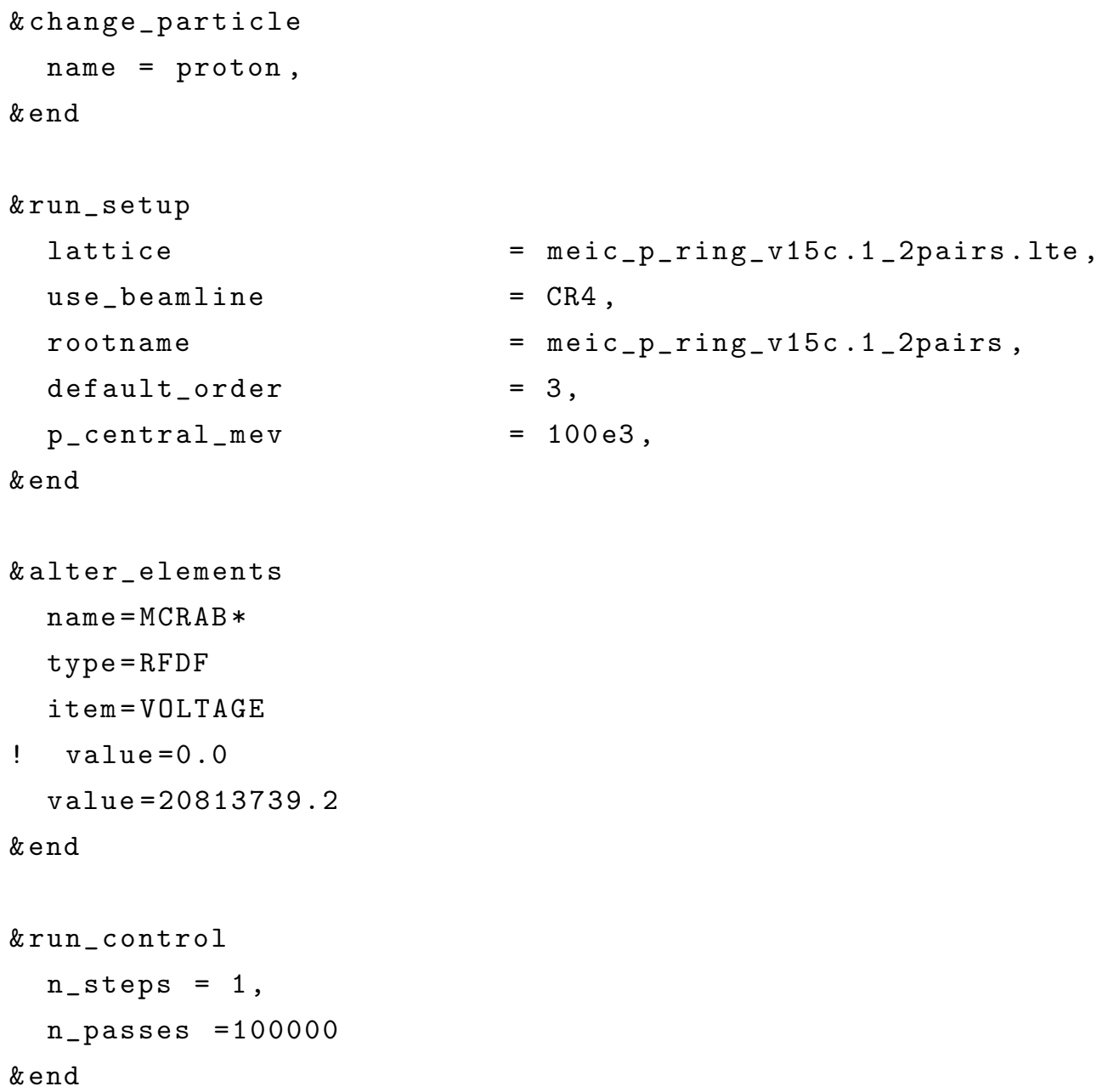




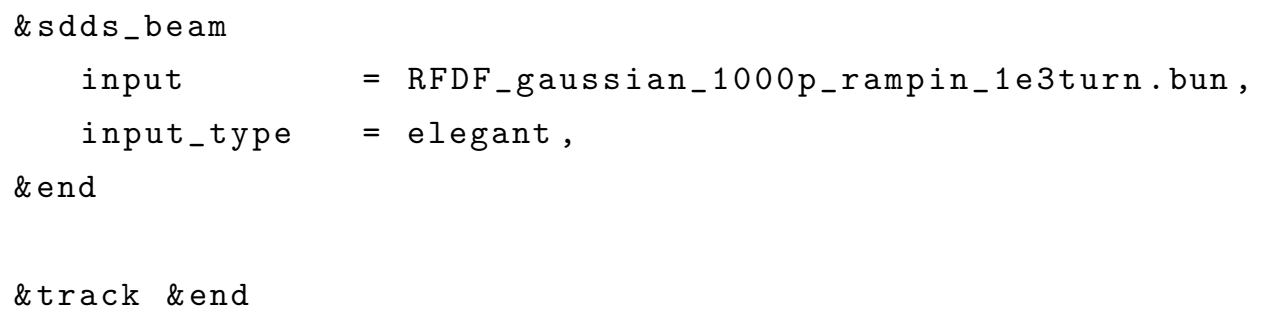

\section{A.3 ELEGANT FILE TO CALCULATE THE DYNAMIC APERTURE.}

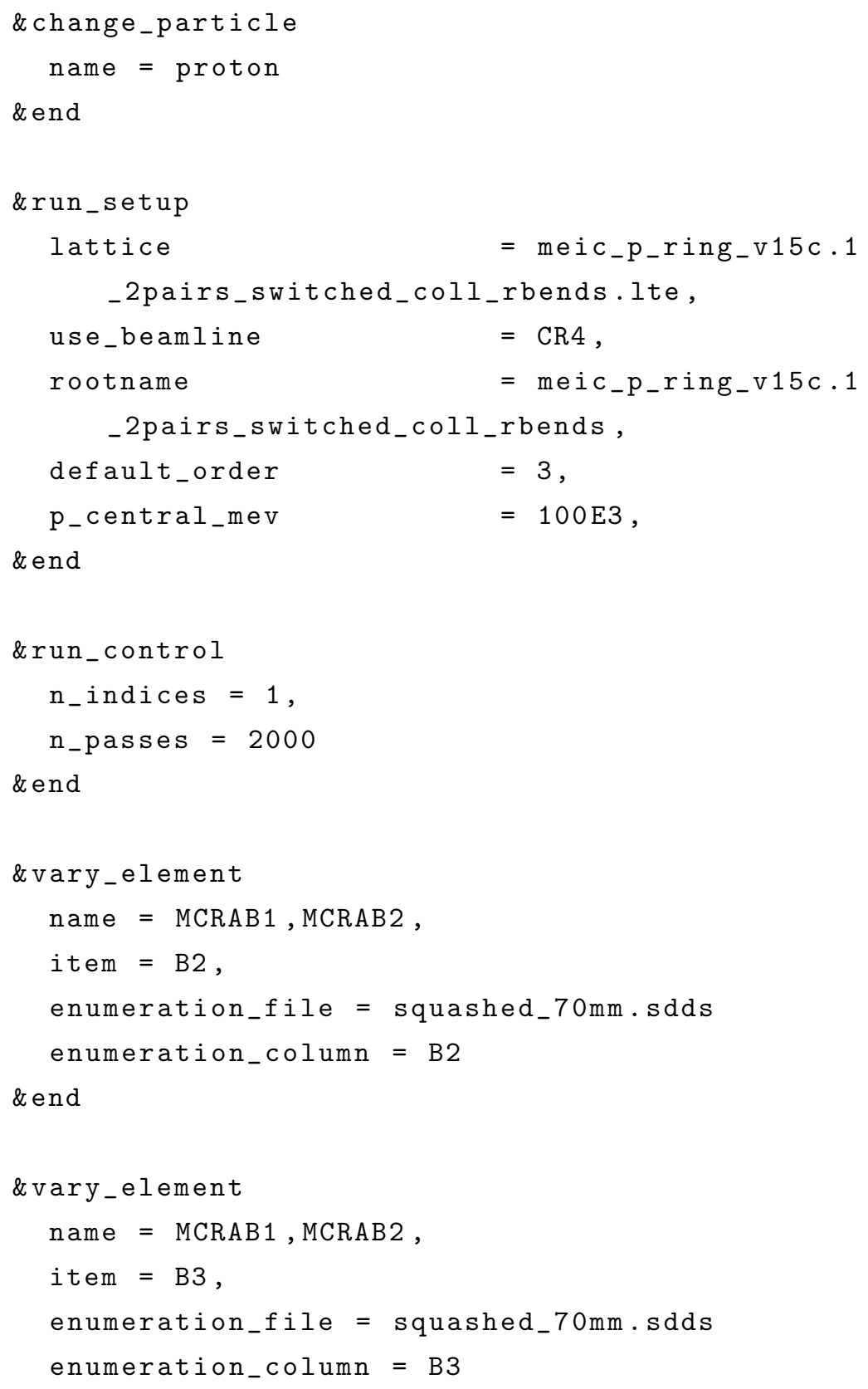




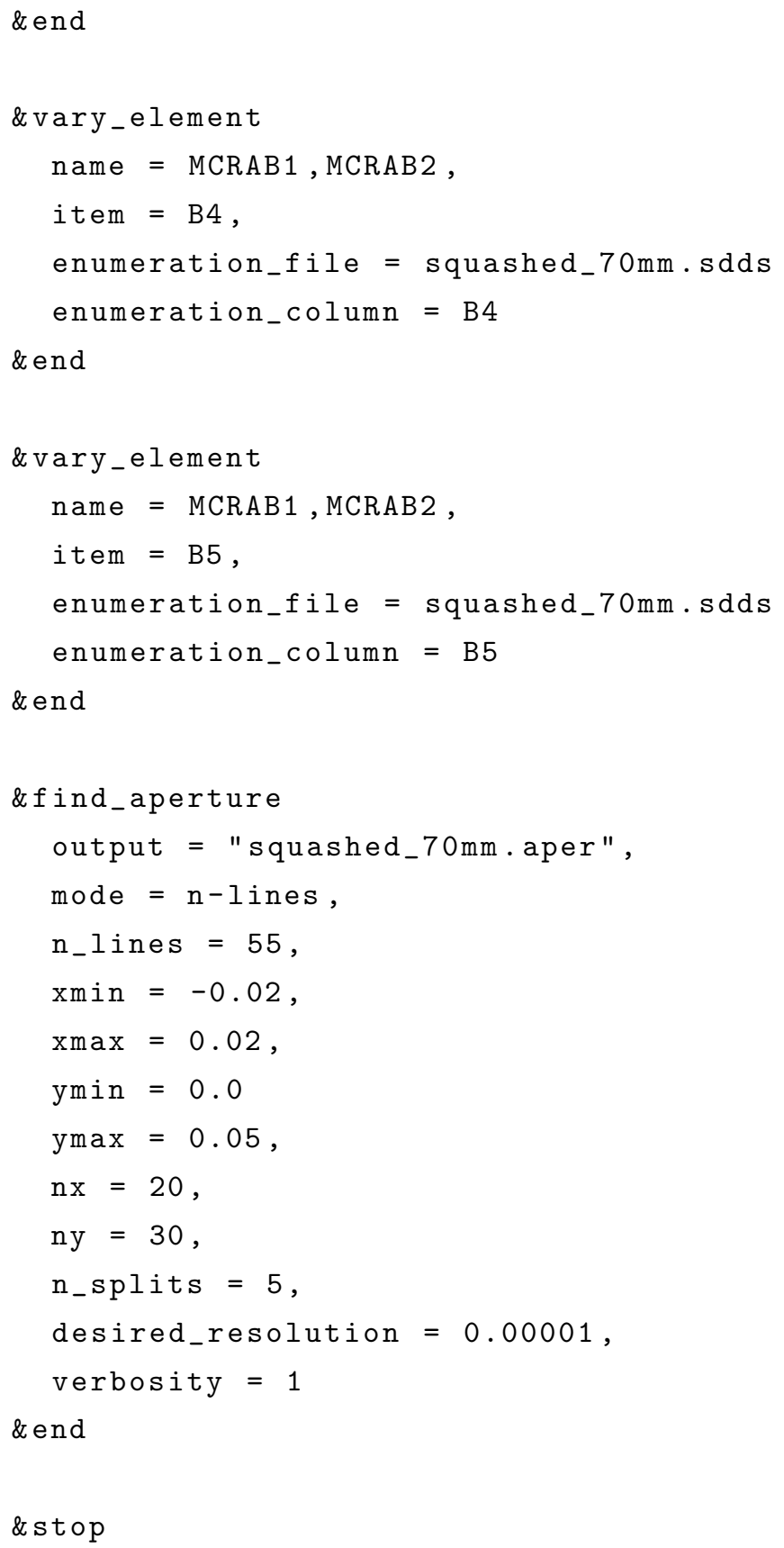

\section{A.4 PYTHON SCRIPT FOR LONGITUDINAL CBI CALCULATION.}

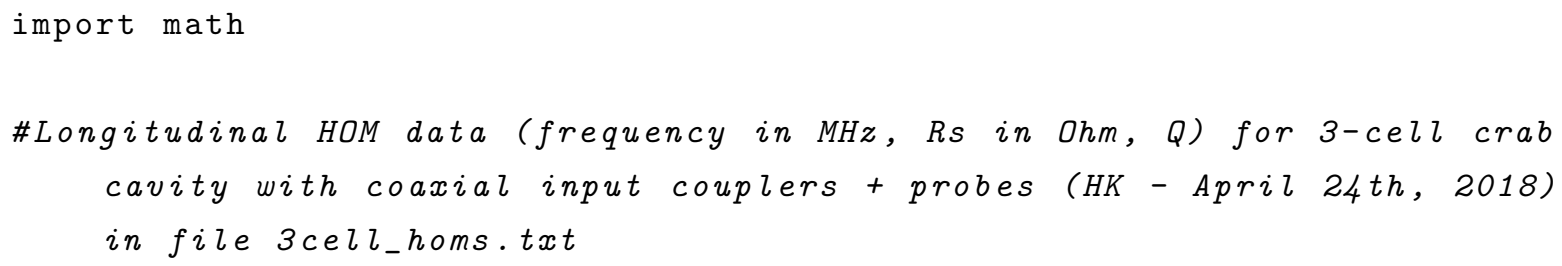




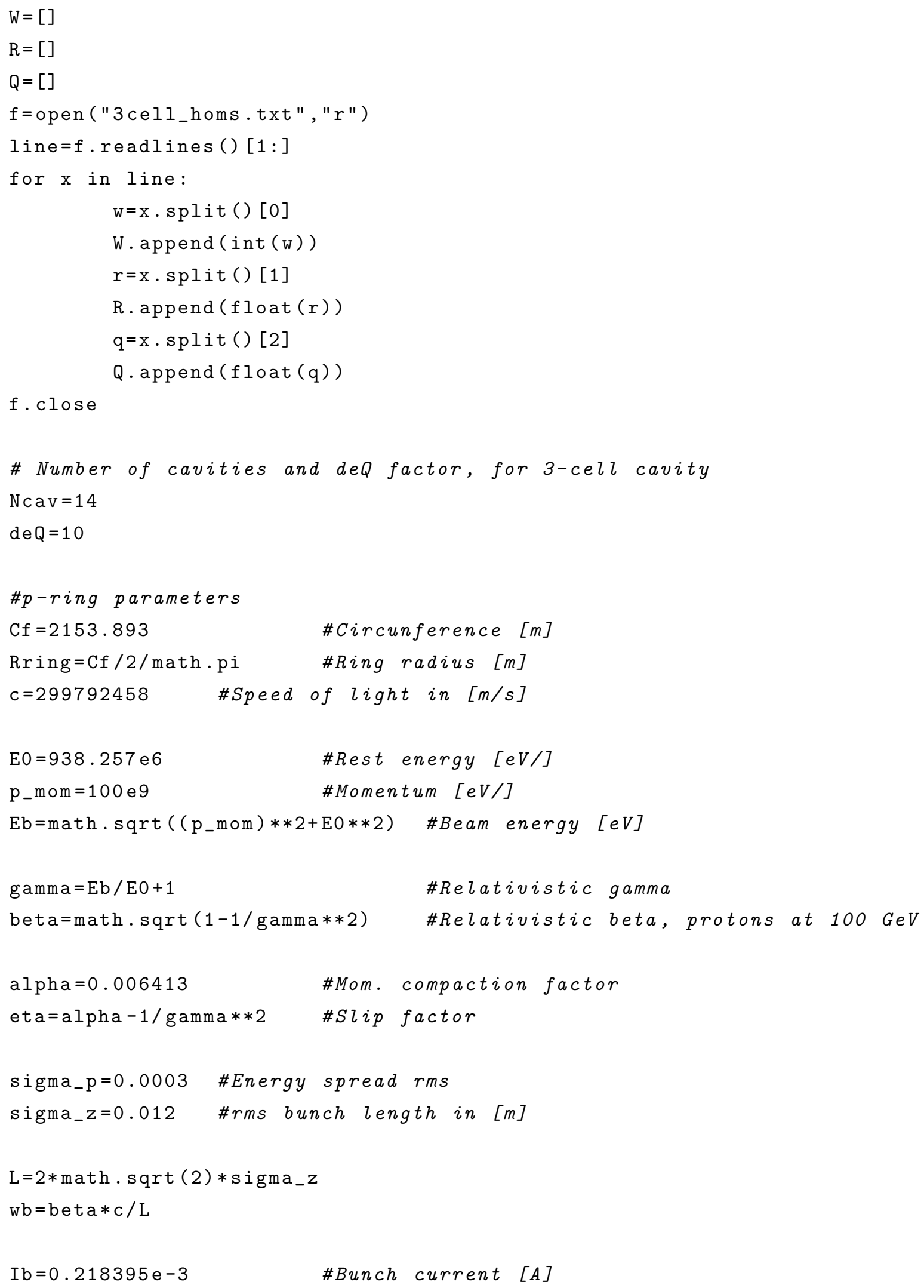




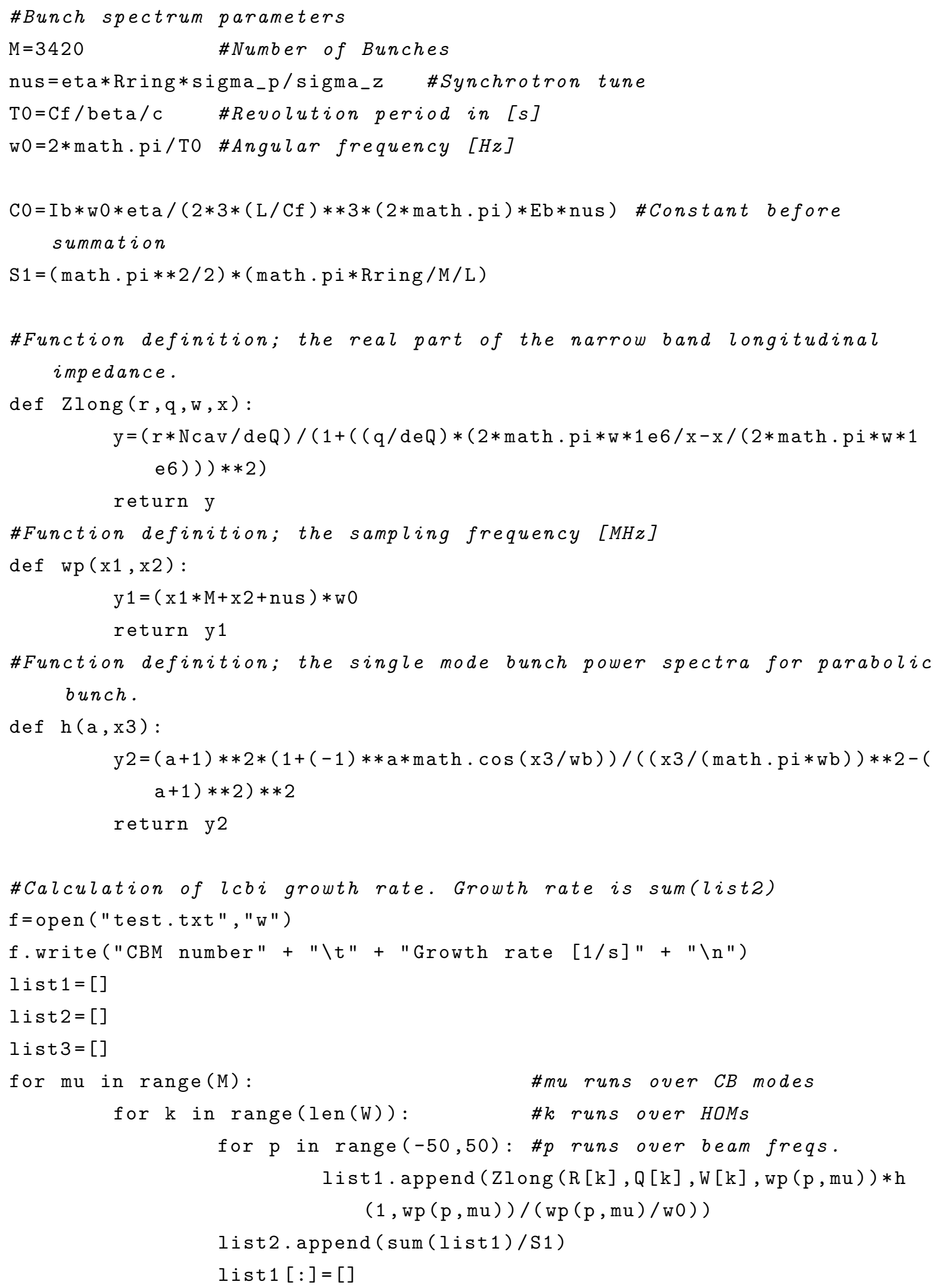


1 ist 3 . append ( $0 * \operatorname{sum}(1$ ist 2$))$

f. write $(\operatorname{str}(m u)+" \backslash t "+\operatorname{str}(1 i s t 3[m u])+" \backslash n ")$

list $2[:]=[]$

f.close ()

\#Print output

print 'Min lcbi growth time is, + str(1000/max(list3)) + ' ms for CBM

,$+\operatorname{str}(1$ ist $3 . \operatorname{index}(\max (1$ ist 3$)))$

\#print 'Growth rate is' $+\operatorname{str}(\max ($ list3)) 


\section{APPENDIX B}

\section{JLEIC RING FILES}

Files in elegant format containing the ion ring baseline lattice, Section B.1, and electron ring lattice, Section B.2. These rings were developed and provided by V. Morozov and F. Lei.

\section{B.1 ION RING}

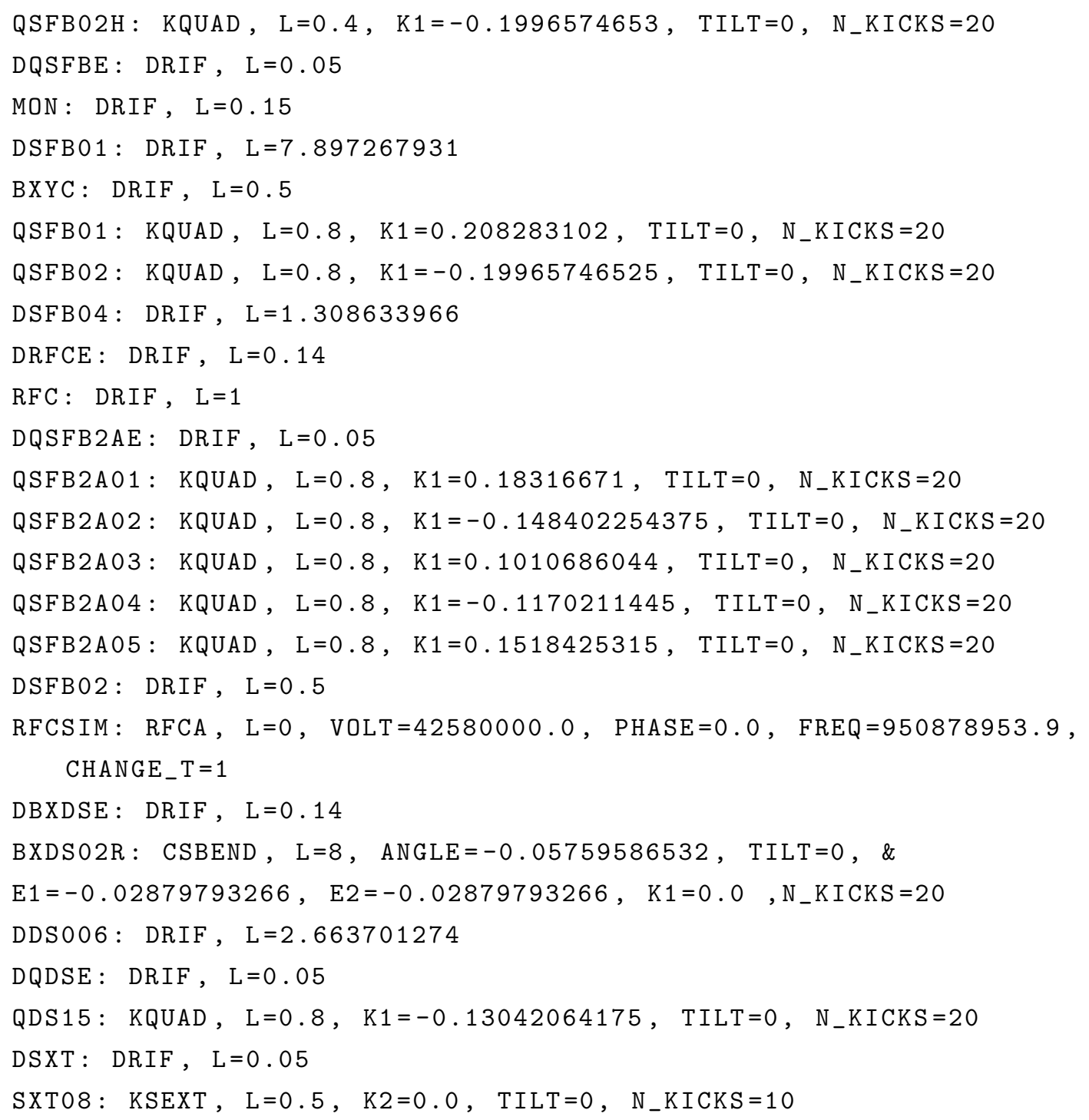




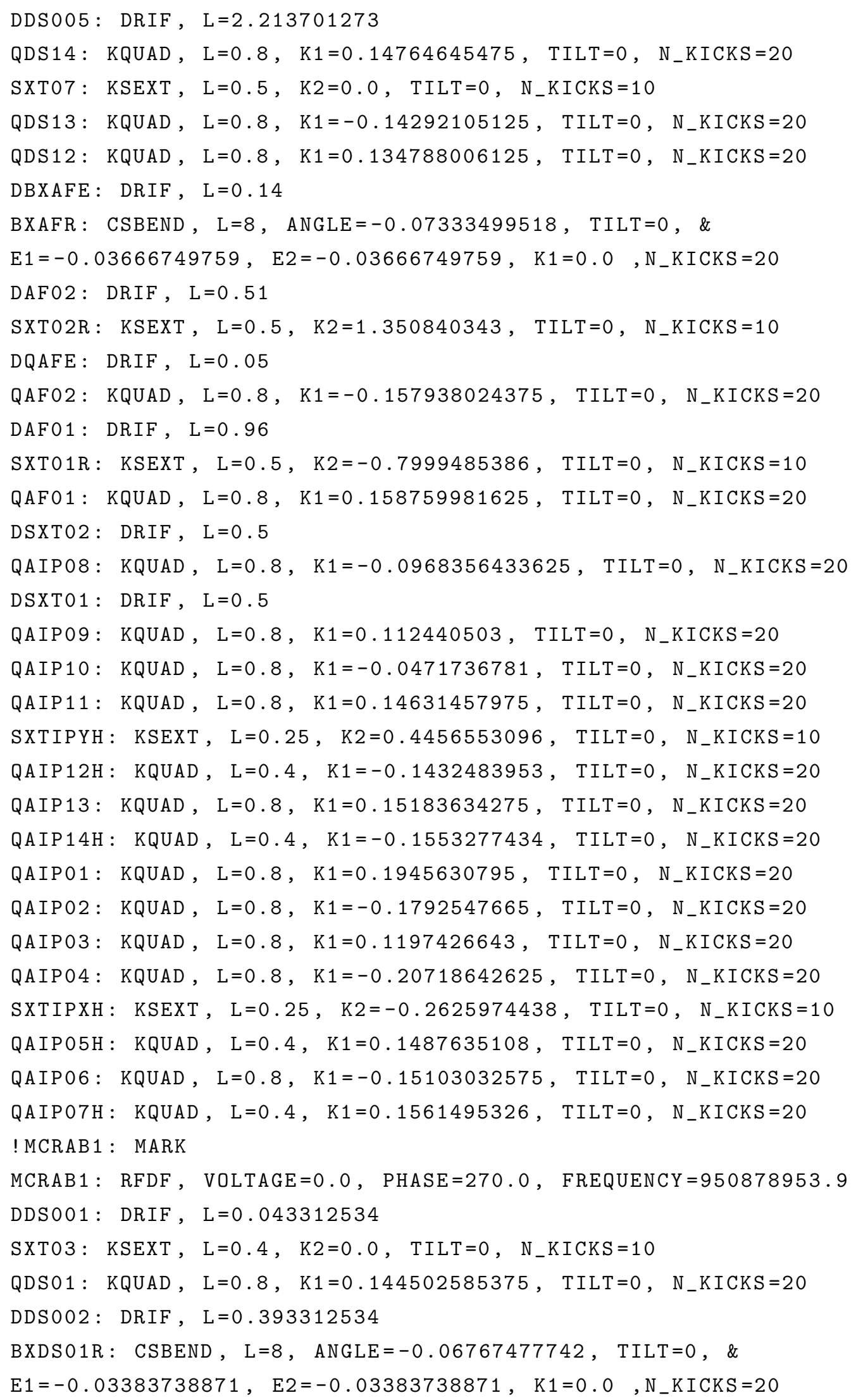




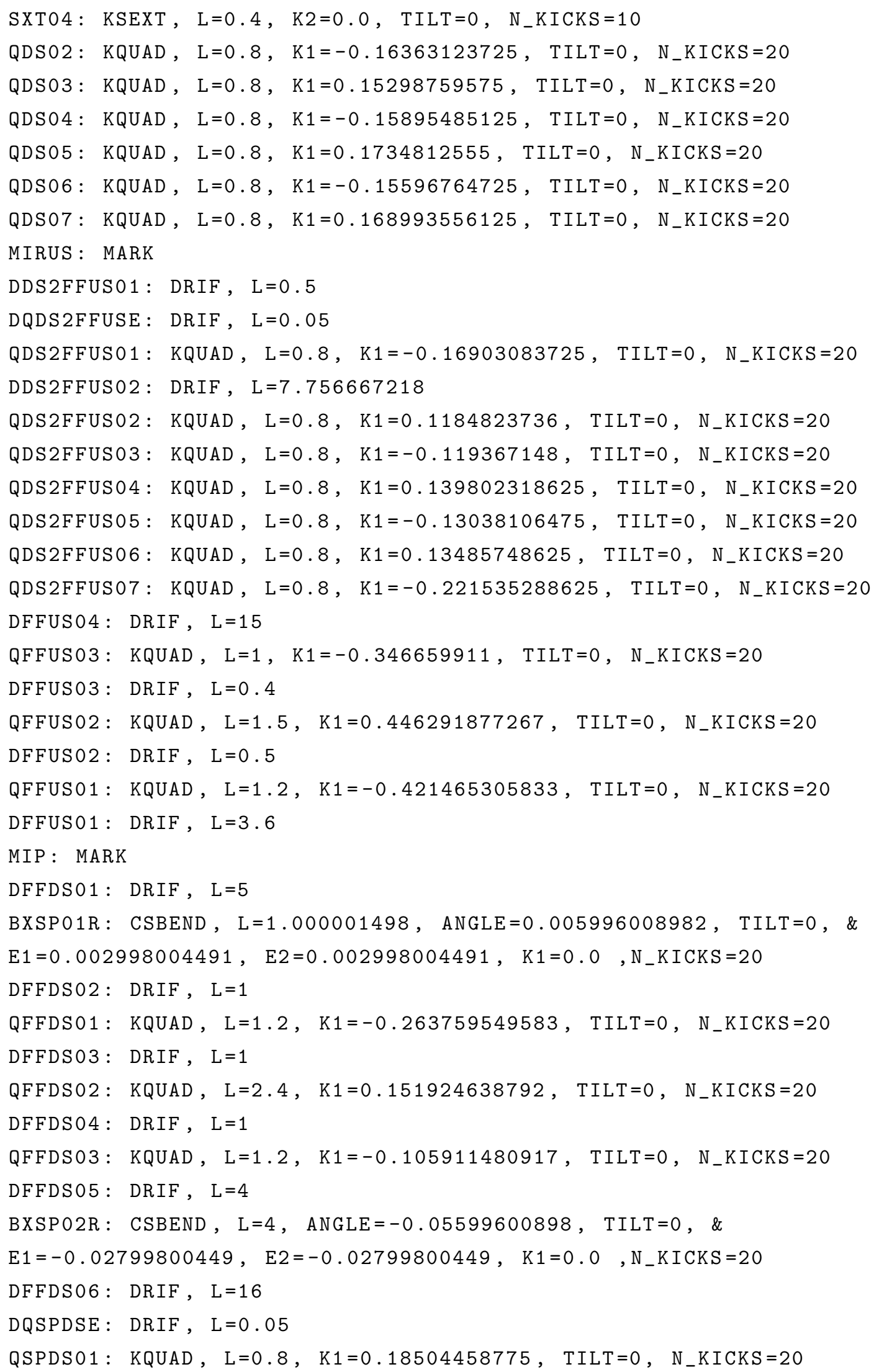




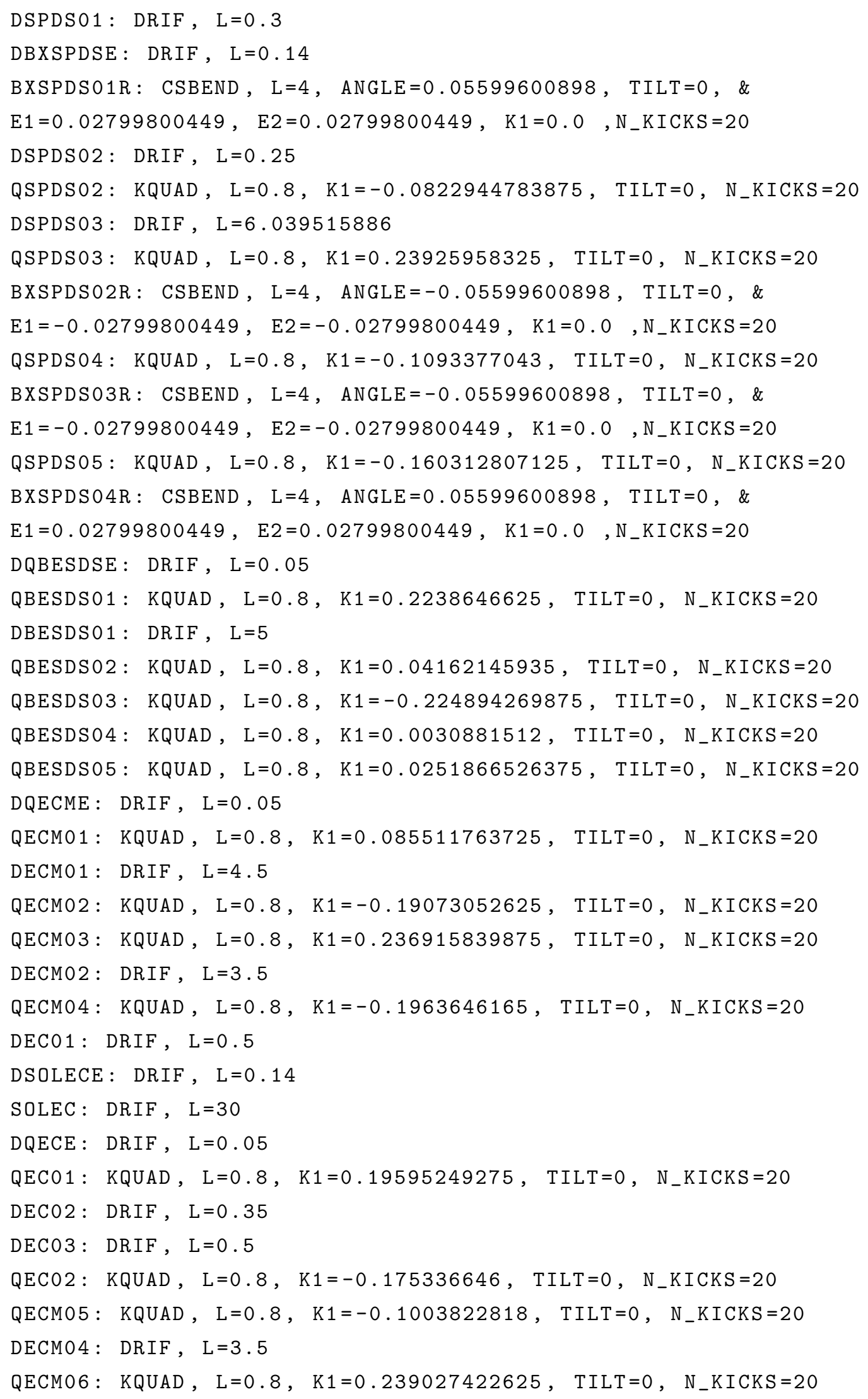




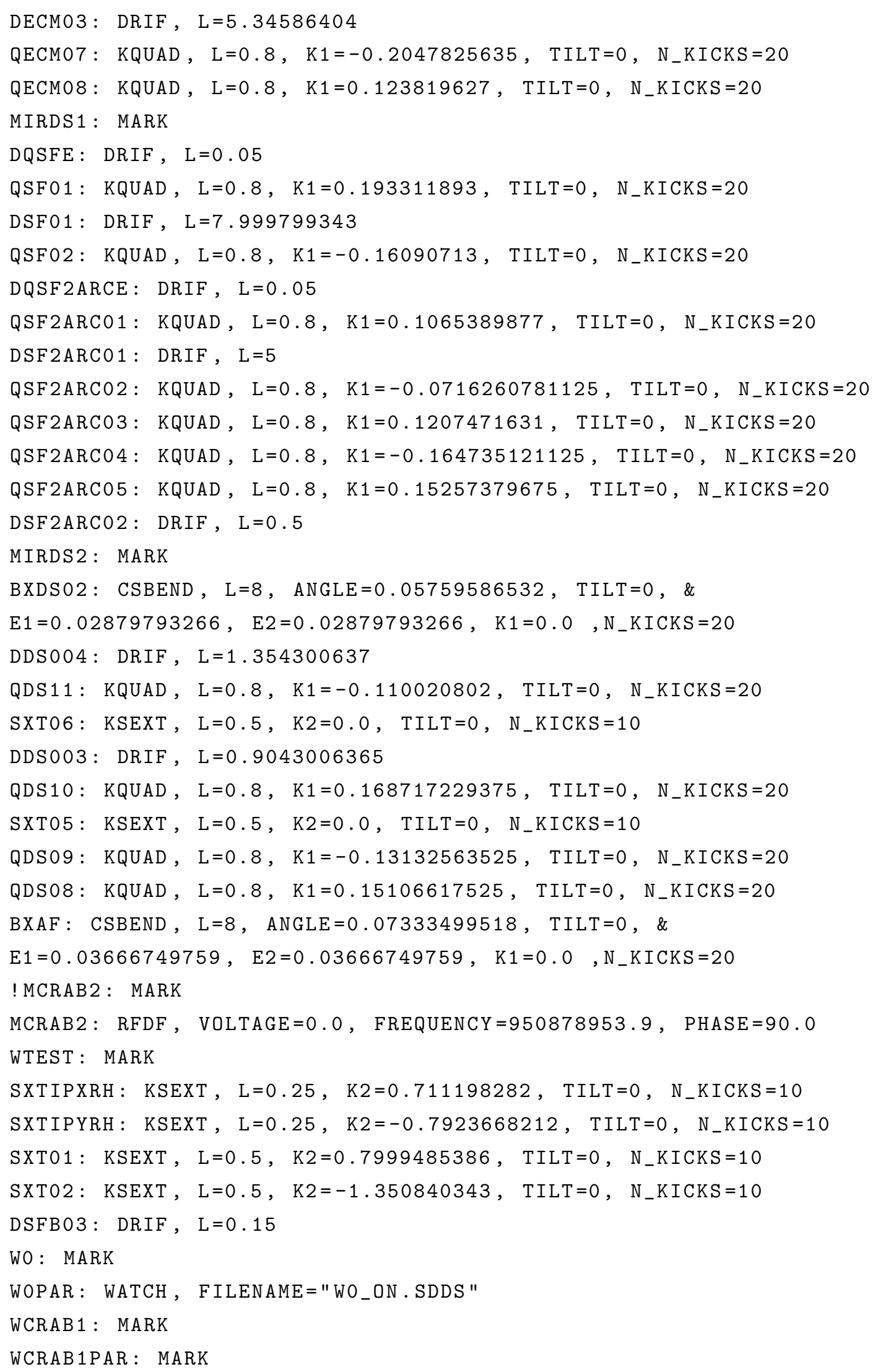


WIP: WATCH, FILENAME $=$ "WIP_ON. SDDS"

WIPPAR : MARK

WCRAB2: MARK

WCRAB2PAR: MARK

W1: MARK

W1PAR: MARK

CR4: LINE $=(\&$

WO, WOPAR, QSFB02H, DQSFBE, MON , DSFB01, BXYC , DQSFBE, QSFB01, DQSFBE, MON , DSFBO1

, \&

BXYC , DQSFBE, QSFB02, DQSFBE, MON , DSFB01, BXYC , DQSFBE, QSFB01, DQSFBE , \&

MON , DSFBO 1, BXYC, DQSFBE, QSFBO2, DQSFBE, MON , DSFBO1, BXYC , DQSFBE , \&

QSFBO 1 , DQSFBE , MON , DSFB04, DRFCE , RFC , RFC , RFC , RFC , RFC , \&

DRFCE , DSFBO4, BXYC, DQSFBE, QSFBO2, DQSFBE, MON , DSFBO4, DRFCE , RFC , \&

RFC , RFC , RFC , RFC , DRFCE , DSFB04, BXYC , DQSFBE , QSFB01, DQSFBE , \&

MON , DSFBO 4 , DRFCE , RFC, RFC , RFC , RFC , RFC, DRFCE , DSFBO 4 , \&

BXYC , DQSFBE , QSFB02, DQSFBE, MON , DSFB04, DRFCE , RFC , RFC , RFC , \&

RFC , RFC , DRFCE , DSFB04, BXYC, DQSFBE, QSFB01, DQSFBE, MON , DSFB04, \&

DRFCE , RFC , RFC , RFC , RFC , RFC , DRFCE , DSFBO4 , BXYC , DQSFBE , \&

QSFB02, DQSFBE, MON , DSFB04, DRFCE , RFC , RFC , RFC , RFC , RFC , \&

DRFCE , DSFB04, BXYC, DQSFBE, QSFB01, DQSFBE, MON, DSFB04, DRFCE, RFC , \&

RFC , RFC , RFC , RFC , DRFCE , DSFB04, BXYC, DQSFBE, QSFBO2, DQSFBE , \&

MON , DSFBO4 , DRFCE , RFC , RFC , RFC , RFC , RFC , DRFCE , DSFBO 4 , \&

BXYC, DQSFB2AE, QSFB2A01, DQSFB2AE, MON , DSFB01, BXYC, DQSFB2AE, QSFB2A02 ,

DQSFB2AE , \&

MON , DSFB01, BXYC, DQSFB2AE, QSFB2A03, DQSFB2AE, MON , DSFB01, BXYC, DQSFB2AE , \&

QSFB2A04, DQSFB2AE, MON , DSFB01, BXYC, DQSFB2AE, QSFB2A05, DQSFB2AE, MON , DSFB02

, \&

RFCSIM , DBXDSE, BXDS02R, DBXDSE, DDS006, MON, DQDSE, QDS15, DQDSE, DSXT , \&

SXT08, DSXT, DDS005, DBXDSE, BXDS02R, DBXDSE, DDS006, MON , DQDSE, QDS 14 , \&

DQDSE , DSXT , SXT07, DSXT, DDS005, DBXDSE, BXDS02R, DBXDSE, DDS006, MON , \&

DQDSE , QDS13, DQDSE, DSXT , SXT08, DSXT , DDS005, DBXDSE, BXDS02R, DBXDSE , \&

DDS006, MON , DQDSE , QDS12, DQDSE , DSXT , SXT07 , DSXT , DDS005 , DBXAFE , \&

BXAFR, DBXAFE, DAFO2, DSXT , SXTO2R, DSXT, DQAFE, QAFO2, DQAFE, MON \& \&

DAF01, DBXAFE , BXAFR, DBXAFE, DAF02, DSXT, SXT01R, DSXT, DQAFE, QAF01, \&

DQAFE, MON , DAF01, DBXAFE, BXAFR, DBXAFE, DAF02, DSXT, SXT02R, DSXT , \&

DQAFE, QAF02, DQAFE, MON , DAF01, DBXAFE , BXAFR, DBXAFE , DAF02, DSXT , \&

SXT01R, DSXT, DQAFE, QAF01, DQAFE, MON , DAF01, DBXAFE, BXAFR , DBXAFE , \&

DAFO2 , DSXT , SXT02R, DSXT, DQAFE, QAF02, DQAFE, MON , DAF01, DBXAFE , \&

BXAFR, DBXAFE, DAF02, DSXT , SXT01R, DSXT, DQAFE, QAF01, DQAFE , MON , \&

DAF01, DBXAFE, BXAFR, DBXAFE, DAF02, DSXT, SXT02R, DSXT, DQAFE, QAF02, \&

DQAFE, MON , DAF01, DBXAFE, BXAFR, DBXAFE, DAF02, DSXT, SXT01R, DSXT, \& 
DQAFE, QAF01, DQAFE, MON , DAF01, DBXAFE, BXAFR, DBXAFE, DAF02, DSXT \& SXT02R, DSXT, DQAFE, QAF02, DQAFE, MON , DAF01, DBXAFE, BXAFR, DBXAFE \& \& DAF02, DSXT, SXT01R, DSXT, DQAFE, QAF01, DQAFE, MON, DAF01, DBXAFE, \& BXAFR, DBXAFE, DAF02, DSXT , SXT02R, DSXT, DQAFE, QAFO2, DQAFE, MON \& \& DAF01, DBXAFE, BXAFR, DBXAFE, DAF02, DSXT, SXT01R, DSXT, DQAFE, QAF01, \& DQAFE , MON , DAF01, DBXAFE, BXAFR, DBXAFE, DAF02, DSXT , SXT02R, DSXT , \& DQAFE, QAF02, DQAFE, MON , DAF01, DBXAFE, BXAFR, DBXAFE, DAF02, DSXT, \& SXT01R, DSXT, DQAFE, QAF01, DQAFE, MON , DAF01, DBXAFE, BXAFR, DBXAFE , \& DAF02, DSXT, SXTO2R, DSXT, DQAFE, QAF02, DQAFE, MON, DAF01, DBXAFE, \& BXAFR, DBXAFE, DAF02, DSXT , SXT01R, DSXT, DQAFE, QAF01, DQAFE, MON \& DAF01, DBXAFE, BXAFR , DBXAFE, DAF02, DSXT , SXT02R, DSXT, DQAFE, QAF02, \& DQAFE, MON , DAF01, DBXAFE, BXAFR, DBXAFE, DAF02, DSXT, SXT01R, DSXT , \& DQAFE, QAF01, DQAFE, MON , DAF01, DBXAFE, BXAFR, DBXAFE, DAF02, DSXT \& SXT02R, DSXT, DQAFE, QAF02, DQAFE, MON, DAF01, DBXAFE, BXAFR, DBXAFE , \& DAF02, DSXT, SXT01R, DSXT, DQAFE, QAF01, DQAFE, MON, DAF01, DBXAFE, \& BXAFR, DBXAFE, DAF02, DSXT , SXT02R, DSXT, DQAFE, QAFO2, DQAFE, MON \& DAF01, DBXAFE, BXAFR, DBXAFE, DAF02, DSXT, SXT01R, DSXT, DQAFE, QAF01, \& DQAFE , MON , DAFO1, DBXAFE , BXAFR, DBXAFE, DAF02, DSXT , SXT02R, DSXT , \& DQAFE, QAF02, DQAFE, MON , DAF01, DBXAFE, BXAFR, DBXAFE, DAF02, DSXT \& SXT01R, DSXT, DQAFE, QAF01, DQAFE, MON , DAF01, DBXAFE, BXAFR, DBXAFE , \& DAF02, DSXT, DSXT02, DSXT, DQAFE, QAIP08, DQAFE, MON , DAF01, DBXAFE , \& BXAFR, DBXAFE, DAF02, DSXT , DSXT01, DSXT , DQAFE, QAIP09, DQAFE, MON , \& DAF01, DBXAFE, BXAFR, DBXAFE, DAF02, DSXT, DSXT02, DSXT, DQAFE, QAIP10, \& DQAFE , MON , DAFO1, DBXAFE , BXAFR, DBXAFE, DAF02, DSXT , DSXT01, DSXT , \& DQAFE , QAIP11, DQAFE, MON , DAF01, DBXAFE, BXAFR, DBXAFE, DAF02, DSXT \& SXTIPYH , SXTIPYH, DSXT, DQAFE, QAIP12H, QAIP12H, DQAFE, MON , DAF01, DBXAFE , \& BXAFR, DBXAFE, DAF02, DSXT, DSXT01, DSXT, DQAFE, QAIP13, DQAFE, MON \& DAF01, DBXAFE, BXAFR, DBXAFE, DAF02, DSXT, DSXT02, DSXT, DQAFE, QAIP14H , \& QAIP14H, DQAFE, MON, DAF01, DBXAFE, BXAFR, DBXAFE, DAF02, DSXT, DSXT01, \& DSXT , DQAFE, QAIP13, DQAFE, MON , DAF01, DBXAFE, BXAFR , DBXAFE, DAF01, \& MON , DQAFE , QAIP12H, QAIP12H, DQAFE, DSXT , SXTIPYH, SXTIPYH , DSXT, DAFO2 \& DBXAFE, BXAFR , DBXAFE, DAF02, DSXT , DSXT01, DSXT, DQAFE, QAIP11, DQAFE , \& MON , DAF01, DBXAFE , BXAFR , DBXAFE, DAF02, DSXT , DSXT02, DSXT , DQAFE , \& QAIP10, DQAFE, MON , DAF01, DBXAFE, BXAFR , DBXAFE, DAF02, DSXT , DSXT01, \& DSXT , DQAFE, QAIP09, DQAFE, MON , DAFO1, DBXAFE, BXAFR, DBXAFE, DAF02, \& DSXT , DSXT02, DSXT, DQAFE, QAIP08, DQAFE, MON , DAF01, DBXAFE, BXAFR \& DBXAFE, DAF02, DSXT , DSXT01, DSXT, DQAFE, QAIP01, DQAFE, MON , DAF01, \& DBXAFE, BXAFR, DBXAFE, DAFO2, DSXT, DSXT02, DSXT, DQAFE, QAIPO2, DQAFE , \& MON , DAF01, DBXAFE, BXAFR, DBXAFE, DAF02, DSXT, DSXT01, DSXT, DQAFE , \& QAIP03, DQAFE, MON, DAF01, DBXAFE, BXAFR, DBXAFE, DAF02, DSXT, DSXT02, \& DSXT, DQAFE, QAIP04, DQAFE, MON, DAFO1, DBXAFE, BXAFR, DBXAFE, DAF02, \& 
DSXT , SXTIPXH , SXTIPXH , DSXT , DQAFE , QAIP05H , QAIP05H , DQAFE , MON , DAF01, \& DBXAFE, BXAFR, DBXAFE, DAF02, DSXT, DSXT02, DSXT, DQAFE, QAIP06, DQAFE , \& MON , DAF01, DBXAFE, BXAFR, DBXAFE, DAF02, DSXT, DSXT01, DSXT, DQAFE, \& QAIP07H , QAIP07H , DQAFE, MON , DAF01, DBXAFE, BXAFR, DBXAFE, DAF02, DSXT \& DSXT02, DSXT, DQAFE, QAIP06, DQAFE, MON , DAF01, DBXAFE, BXAFR , DBXAFE, \& DAF01, MON , DQAFE, QAIP05H, QAIP05H, DQAFE, DSXT, SXTIPXH , SXTIPXH , DSXT , \& MCRAB1, WCRAB1, WCRAB1PAR, DAF02, DBXAFE, BXAFR, DBXAFE, DAF02, DSXT, DSXT02, DSXT , DQAFE , \& QAIP04, DQAFE , MON , DAF01, DBXAFE , BXAFR, DBXAFE , DAF02, DSXT , DSXT01, \& DSXT , DQAFE , QAIP03, DQAFE, MON , DAF01, DBXAFE, BXAFR, DBXAFE , DAF02, \& DSXT , DSXTO2, DSXT, DQAFE, QAIP02, DQAFE, MON , DAF01, DBXAFE , BXAFR , \& DBXAFE , DAF02, DSXT , DSXT01, DSXT, DQAFE, QAIP01, DQAFE, MON , DAF01, \& DBXAFE , BXAFR, DBXAFE, DAF02, DSXT, DSXT02, DSXT, DQAFE, QAF02, DQAFE, \& MON , DAF01, DBXAFE , BXAFR , DBXAFE, DDS001, DSXT , SXT03, DSXT , DQDSE , \& QDS01, DQDSE, MON , DDS002, DBXDSE, BXDS01R, DBXDSE, DDS001, DSXT , SXT04,\& DSXT , DQDSE , QDSO2, DQDSE , MON , DDS002, DBXDSE , BXDS01R , DBXDSE , DDS001, \& DSXT , SXT03, DSXT , DQDSE , QDS03, DQDSE , MON , DDS002, DBXDSE , BXDS01R , \& DBXDSE , DDS001, DSXT , SXT04, DSXT , DQDSE, QDS04, DQDSE , MON , DDS002, \& DBXDSE, BXDS01R, DBXDSE, DDS001, DSXT , SXT03, DSXT, DQDSE, QDS05, DQDSE, \& MON , DDS002, DBXDSE , BXDS01R, DBXDSE, DDS001, DSXT , SXT04, DSXT , DQDSE , \& QDS06, DQDSE, MON , DDS002, DBXDSE, BXDS01R, DBXDSE, DDS001, DSXT, SXT03,\& DSXT , DQDSE , QDS07, DQDSE , MON , DDS002, DBXDSE , BXDS01R, DBXDSE, MIRUS , \& DDS2FFUS01, BXYC , DQDS2FFUSE, QDS2FFUS01, DQDS2FFUSE, MON , DDS2FFUSO2, BXYC , DQDS2FFUSE, QDS2FFUS02, \&

DQDS2FFUSE , MON , DDS2FFUSO2, BXYC, DQDS2FFUSE, QDS2FFUS03, DQDS2FFUSE , MON , DDS2FFUSO2, BXYC , \&

DQDS2FFUSE, QDS2FFUS04, DQDS2FFUSE, MON , DDS2FFUSO2, BXYC, DQDS2FFUSE , QDS2FFUS05, DQDS2FFUSE , MON , \&

DDS2FFUS02, BXYC , DQDS2FFUSE , QDS2FFUS06, DQDS2FFUSE , MON , DDS2FFUS02, BXYC , DQDS2FFUSE, QDS2FFUS07, \&

DQDS2FFUSE, DQDS2FFUSE, QDS2FFUS07, DQDS2FFUSE, MON , DFFUS04, QFFUS03, DFFUSO3 , QFFUS02, DFFUSO2, \&

QFFUS01, DFFUS01, MIP ,WIP ,WIPPAR, DFFDS01, BXSP01R, DFFDS02, QFFDS01, DFFDS03 , QFFDSO2, DFFDS04, \&

QFFDS03,DFFDS05 , BXSP02R , DFFDS06 , BXYC , DQSPDSE , QSPDS01, DQSPDSE , MON , DSPDS01, \&

DBXSPDSE , BXSPDS01R , DBXSPDSE , DSPDS02, BXYC , DQSPDSE , QSPDSO2 , DQSPDSE , MON , DSPDSO3,\&

BXYC , DQSPDSE , QSPDS03, DQSPDSE , MON , DSPDS01, DBXSPDSE , BXSPDSO2R , DBXSPDSE , DSPDSO 2 , \& 


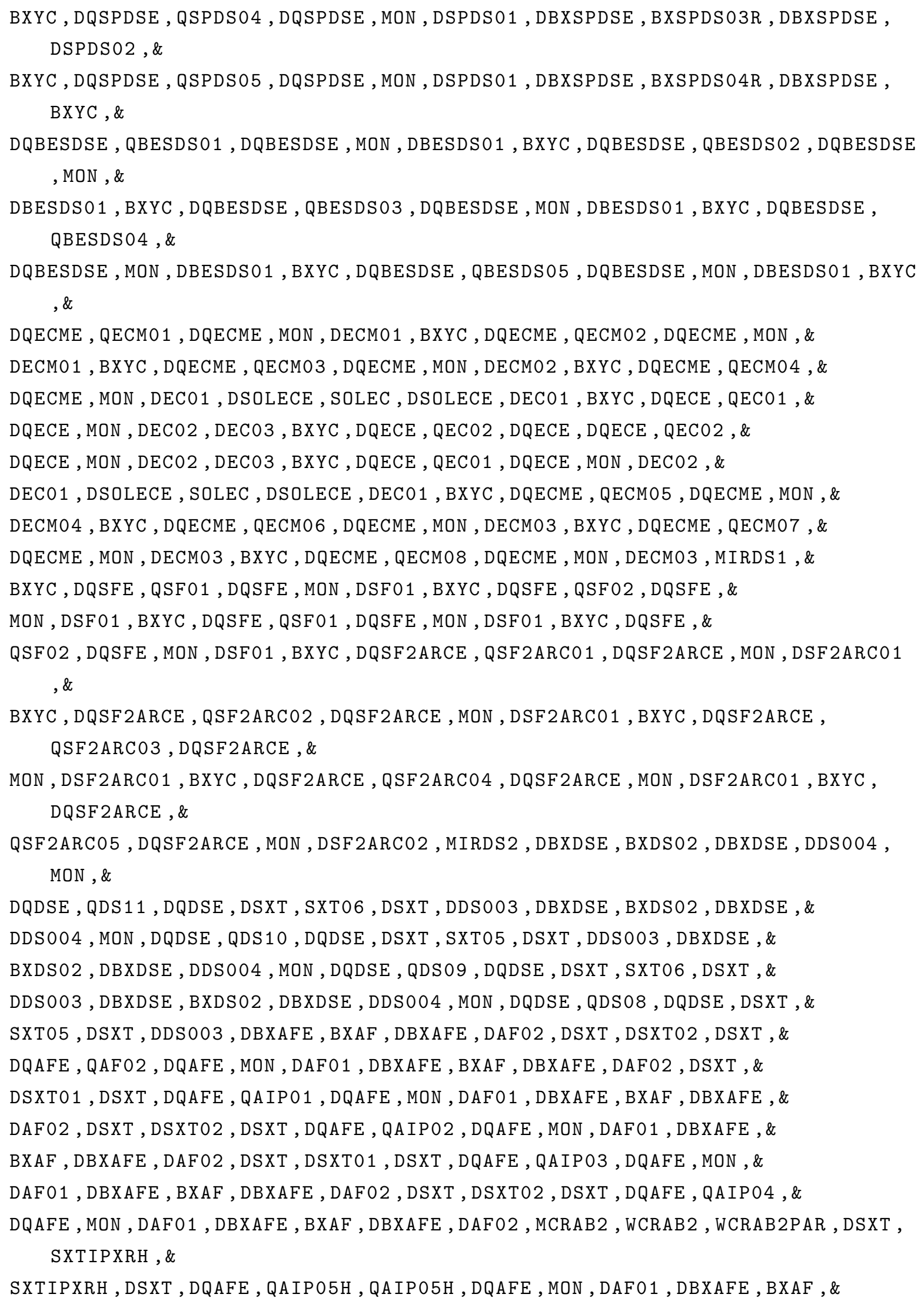


DBXAFE , DAF02, DSXT , DSXT02, DSXT , DQAFE, QAIP06, DQAFE, MON , DAF01, \& DBXAFE , BXAF, DBXAFE , DAF02, DSXT , DSXT01, DSXT , DQAFE, QAIP07H, QAIP07H , \& DQAFE, MON , DAF01, DBXAFE, BXAF, DBXAFE, DAFO2, DSXT, DSXT02, DSXT \& \& DQAFE , QAIP06, DQAFE, MON , DAF01, DBXAFE , BXAF , DBXAFE , DAF01, MON \& \& DQAFE, QAIP05H, QAIP05H, DQAFE, DSXT , SXTIPXRH , SXTIPXRH , DSXT ,WTEST , DAF02, DBXAFE , \&

BXAF, DBXAFE , DAF02, DSXT , DSXT02, DSXT , DQAFE, QAIP04, DQAFE , MON \& \& DAF01, DBXAFE, BXAF, DBXAFE, DAF02, DSXT, DSXT01, DSXT, DQAFE, QAIP03 \& \& DQAFE, MON , DAF01, DBXAFE, BXAF, DBXAFE, DAF02, DSXT, DSXT02, DSXT, \& DQAFE , QAIP02, DQAFE, MON , DAF01, DBXAFE, BXAF, DBXAFE, DAF02, DSXT , \& DSXT01, DSXT, DQAFE, QAIP01, DQAFE, MON , DAFO1, DBXAFE, BXAF, DBXAFE , \& DAF02, DSXT , DSXT02, DSXT, DQAFE, QAIP08, DQAFE, MON , DAF01, DBXAFE , \& BXAF, DBXAFE , DAF02, DSXT , DSXT01, DSXT, DQAFE, QAIP09, DQAFE, MON \& \& DAF01, DBXAFE, BXAF, DBXAFE, DAF02, DSXT, DSXT02, DSXT, DQAFE, QAIP10, \& DQAFE, MON , DAF01, DBXAFE, BXAF, DBXAFE, DAF02, DSXT, DSXT01, DSXT, \& DQAFE , QAIP11, DQAFE , MON , DAF01, DBXAFE , BXAF, DBXAFE , DAF02, DSXT , \& SXTIPYRH , SXTIPYRH , DSXT , DQAFE, QAIP12H, QAIP12H, DQAFE, MON , DAF01, DBXAFE , \& BXAF, DBXAFE, DAF02, DSXT , DSXT01, DSXT , DQAFE, QAIP13, DQAFE, MON , \& DAFO1, DBXAFE, BXAF, DBXAFE, DAF02, DSXT, DSXT02, DSXT, DQAFE, QAIP14H \& QAIP14H, DQAFE, MON , DAF01, DBXAFE, BXAF, DBXAFE, DAF02, DSXT , DSXT01, \& DSXT , DQAFE, QAIP13, DQAFE, MON , DAF01, DBXAFE, BXAF, DBXAFE, DAF01, \& MON , DQAFE , QAIP12H , QAIP12H, DQAFE, DSXT , SXTIPYRH, SXTIPYRH, DSXT , DAF02, \& DBXAFE, BXAF, DBXAFE, DAF02, DSXT, DSXT01, DSXT, DQAFE, QAIP11, DQAFE , \& MON , DAF01, DBXAFE, BXAF, DBXAFE, DAF02, DSXT, DSXT02, DSXT, DQAFE, \& QAIP10, DQAFE, MON , DAF01, DBXAFE, BXAF, DBXAFE, DAFO2, DSXT, DSXT01, \& DSXT , DQAFE , QAIP09, DQAFE, MON , DAF01, DBXAFE, BXAF, DBXAFE, DAF02, \& DSXT, DSXT02, DSXT, DQAFE, QAIP08, DQAFE, MON, DAF01, DBXAFE, BXAF, \& DBXAFE , DAF02, DSXT , SXT01, DSXT , DQAFE , QAF01, DQAFE, MON , DAF01, \& DBXAFE , BXAF, DBXAFE, DAF02, DSXT , SXT02, DSXT, DQAFE, QAF02, DQAFE , \& MON , DAF01, DBXAFE, BXAF, DBXAFE, DAF02, DSXT , SXT01, DSXT , DQAFE \& \& QAF01, DQAFE, MON, DAF01, DBXAFE, BXAF, DBXAFE, DAF02, DSXT, SXT02, \& DSXT , DQAFE, QAFO2, DQAFE, MON, DAFO1, DBXAFE, BXAF, DBXAFE, DAFO2, \& DSXT , SXT01, DSXT, DQAFE, QAF01, DQAFE, MON , DAF01, DBXAFE, BXAF , \& DBXAFE, DAF02, DSXT , SXT02, DSXT, DQAFE, QAF02, DQAFE, MON , DAF01, \& DBXAFE , BXAF, DBXAFE , DAFO2, DSXT , SXT01, DSXT, DQAFE, QAF01, DQAFE , \& MON , DAF01, DBXAFE, BXAF, DBXAFE, DAF02, DSXT, SXT02, DSXT, DQAFE , \& QAF02, DQAFE, MON, DAF01, DBXAFE, BXAF, DBXAFE, DAF02, DSXT, SXT01, \& DSXT, DQAFE, QAF01, DQAFE, MON, DAFO1, DBXAFE, BXAF, DBXAFE, DAFO2, \& DSXT , SXT02, DSXT, DQAFE, QAF02, DQAFE, MON, DAF01, DBXAFE, BXAF , \& DBXAFE, DAF02, DSXT , SXT01, DSXT, DQAFE, QAF01, DQAFE, MON , DAF01, \& DBXAFE, BXAF, DBXAFE, DAF02, DSXT, SXT02, DSXT, DQAFE, QAF02, DQAFE, \& 
MON , DAF01, DBXAFE , BXAF, DBXAFE, DAF02, DSXT, SXT01, DSXT, DQAFE , \& QAF01, DQAFE, MON , DAF01, DBXAFE, BXAF, DBXAFE, DAF02, DSXT , SXT02, \& DSXT , DQAFE , QAF02, DQAFE, MON , DAF01, DBXAFE, BXAF, DBXAFE, DAF02, \& DSXT , SXT01, DSXT , DQAFE, QAF01, DQAFE, MON , DAF01, DBXAFE, BXAF , \& DBXAFE, DAF02, DSXT , SXT02, DSXT, DQAFE, QAF02, DQAFE, MON , DAF01, \& DBXAFE, BXAF, DBXAFE, DAF02, DSXT, SXT01, DSXT, DQAFE, QAF01, DQAFE , \& MON , DAF01, DBXAFE, BXAF, DBXAFE, DAF02, DSXT, SXT02, DSXT, DQAFE, \& QAF02, DQAFE, MON , DAF01, DBXAFE, BXAF, DBXAFE, DAF02, DSXT, SXT01, \& DSXT, DQAFE, QAF01, DQAFE, MON , DAF01, DBXAFE, BXAF, DBXAFE, DAF02, \& DSXT , SXT02, DSXT, DQAFE, QAF02, DQAFE, MON , DAF01, DBXAFE, BXAF , \& DBXAFE , DAF02, DSXT , SXT01, DSXT, DQAFE, QAF01, DQAFE, MON , DAF01, \& DBXAFE , BXAF, DBXAFE, DAF02, DSXT, SXT02, DSXT, DQAFE, QAF02, DQAFE, \& MON , DAF01, DBXAFE, BXAF, DBXAFE, DAF02, DSXT, SXT01, DSXT, DQAFE, \& QAF01, DQAFE, MON , DAF01, DBXAFE, BXAF, DBXAFE, DAF02, DSXT , SXT02, \& DSXT , DQAFE , QAF02, DQAFE, MON , DAF01, DBXAFE, BXAF, DBXAFE, DAF02, \& DSXT , DSXT01, DSXT, DQAFE, QAF01, DQAFE, MON , DAF01, DBXAFE, BXAF , \& DBXAFE , DAF02, DSXT, DSXT02, DSXT, DQAFE, QAFO2, DQAFE, MON , DAF01, \& DBXAFE , BXAF, DBXAFE, DAF02, DSXT, DSXT01, DSXT, DQAFE, QAF01, DQAFE, \& MON , DAF01, DBXAFE, BXAF, DBXAFE, DAF02, DSXT, DSXT02, DSXT, DQAFE, \& QAF02, DQAFE , MON , DAF01, DBXAFE , BXAF , DBXAFE, DDS005, DSXT, SXT07, \& DSXT , DQDSE , QDS12, DQDSE , MON , DDS006, DBXDSE , BXDS02, DBXDSE , DDS005 \& DSXT , SXT08, DSXT , DQDSE, QDS13, DQDSE, MON , DDS006, DBXDSE , BXDS02, \& DBXDSE , DDS005, DSXT , SXT07, DSXT , DQDSE, QDS14, DQDSE , MON , DDS006 \& DBXDSE , BXDS02, DBXDSE, DDS005, DSXT , SXT08, DSXT , DQDSE, QDS15, DQDSE , \& MON , DDS006, DBXDSE , BXDS02, DBXDSE, DSFB03, BXYC, DQSFB2AE, QSFB2A05, DQSFB2AE , \&

MON , DSFB01, BXYC , DQSFB2AE, QSFB2A04, DQSFB2AE, MON , DSFBO1, BXYC, DQSFB2AE, \& QSFB2A03, DQSFB2AE, MON , DSFB01, BXYC, DQSFB2AE, QSFB2A02, DQSFB2AE, MON, DSFBO1 , \&

BXYC, DQSFB2AE, QSFB2A01, DQSFB2AE, MON , DSFB01, BXYC, DQSFBE, QSFB02, DQSFBE , \& MON , DSFB01, BXYC , DQSFBE , QSFB01, DQSFBE , MON , DSFB01, BXYC , DQSFBE , \& QSFBO2, DQSFBE , MON , DSFB01, BXYC , DQSFBE , QSFB01, DQSFBE , MON , DSFB01, \& BXYC , DQSFBE , QSFB02, DQSFBE , MON , DSFB01, BXYC, DQSFBE , QSFBO1, DQSFBE , \& MON , DSFBO1, BXYC , DQSFBE , QSFBO2, DQSFBE , MON , DSFB01, BXYC, DQSFBE , \& QSFB01, DQSFBE , MON , DSFB01, BXYC, DQSFBE , QSFB02, DQSFBE , MON , DSFB01, \& BXYC , DQSFBE , QSFB01, DQSFBE, MON , DSFB01, BXYC , DQSFBE , QSFBO2, DQSFBE , \& MON , DSFBO1, BXYC , DQSFBE , QSFB01, DQSFBE , MON , DSFBO1, BXYC, DQSFBE , \& QSFB02H, W1, W1PAR )

$\mathrm{USE}, \mathrm{CR} 4$

RETURN 


\section{B.2 ELECTRON RING}

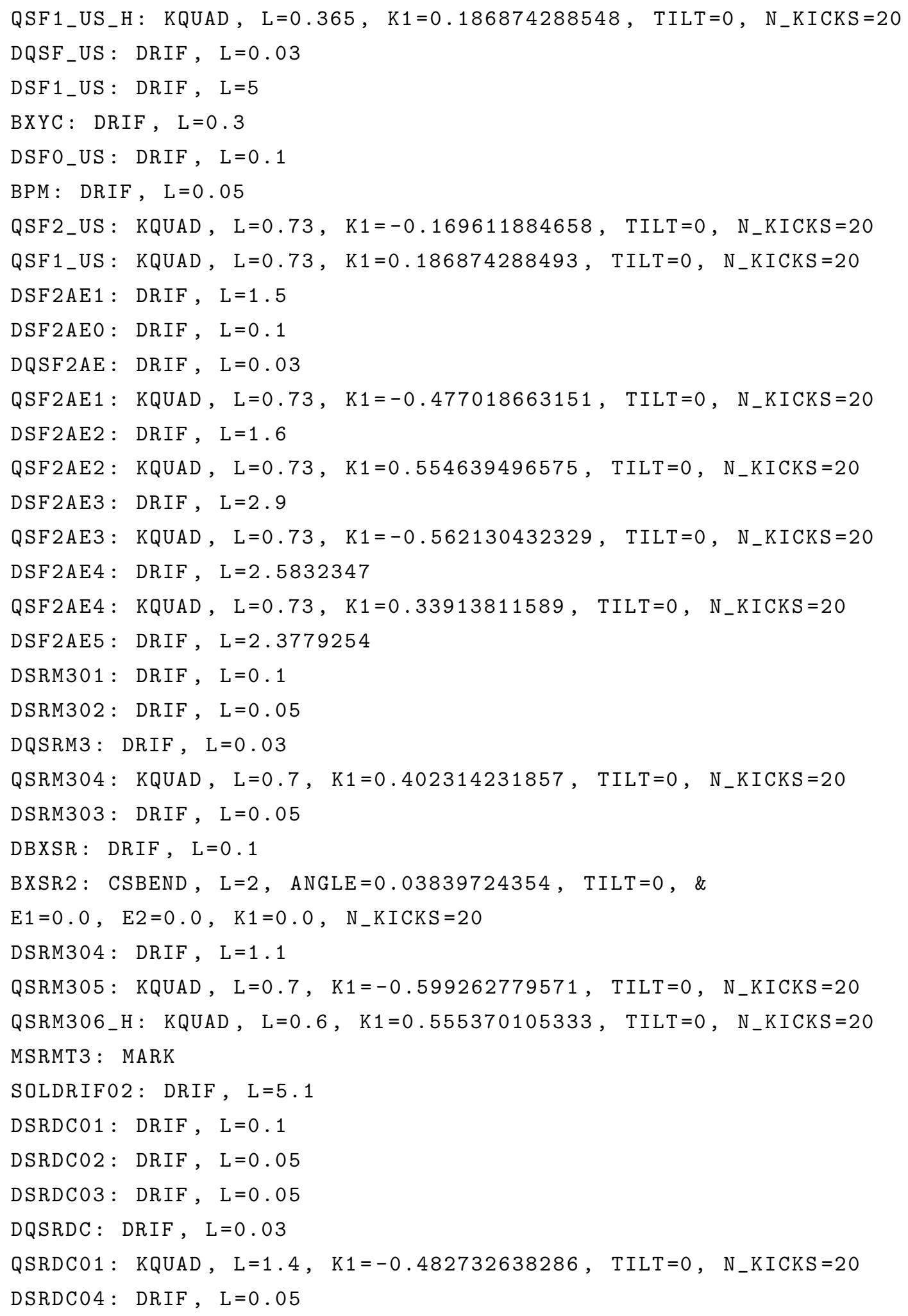




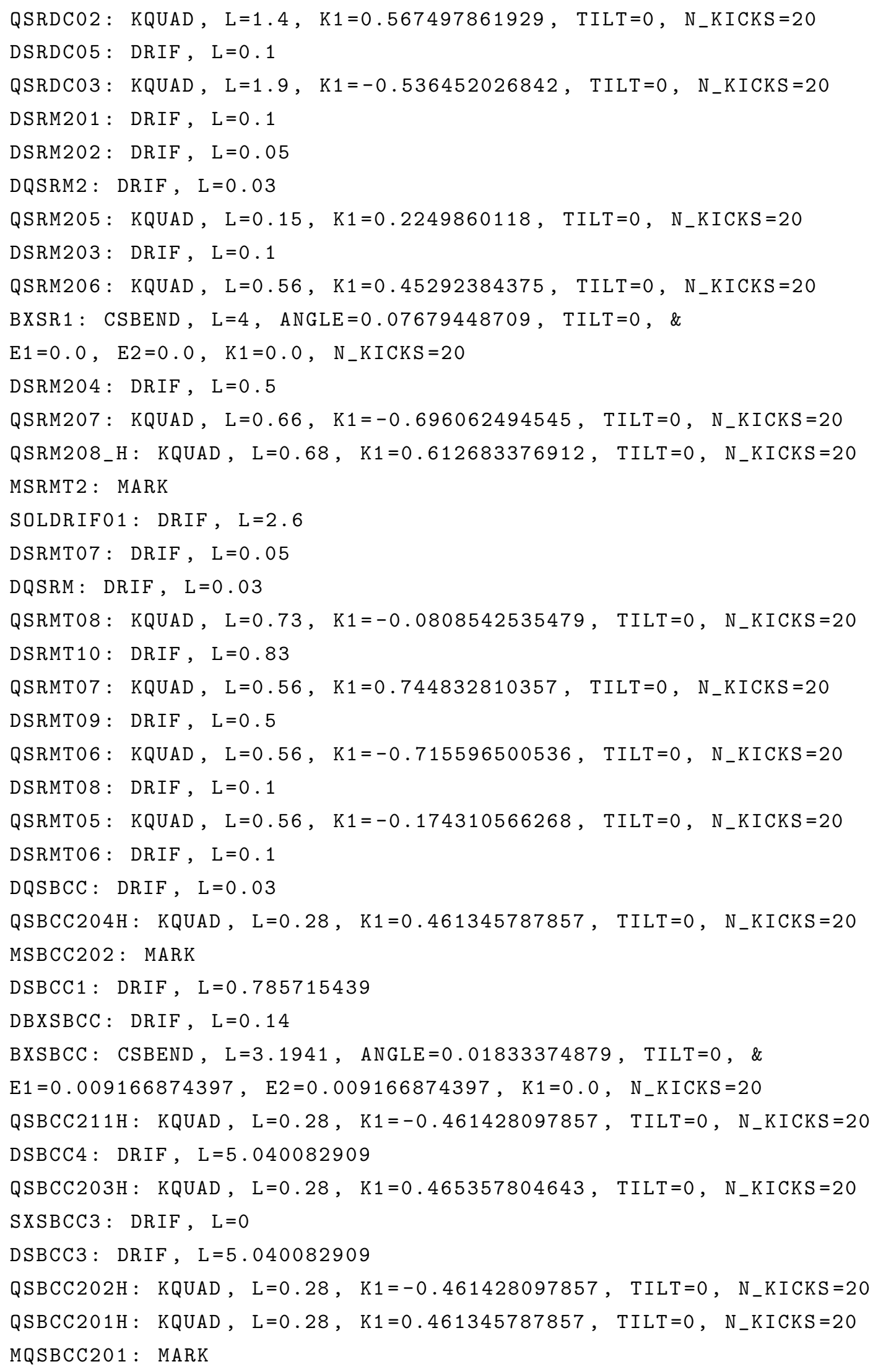




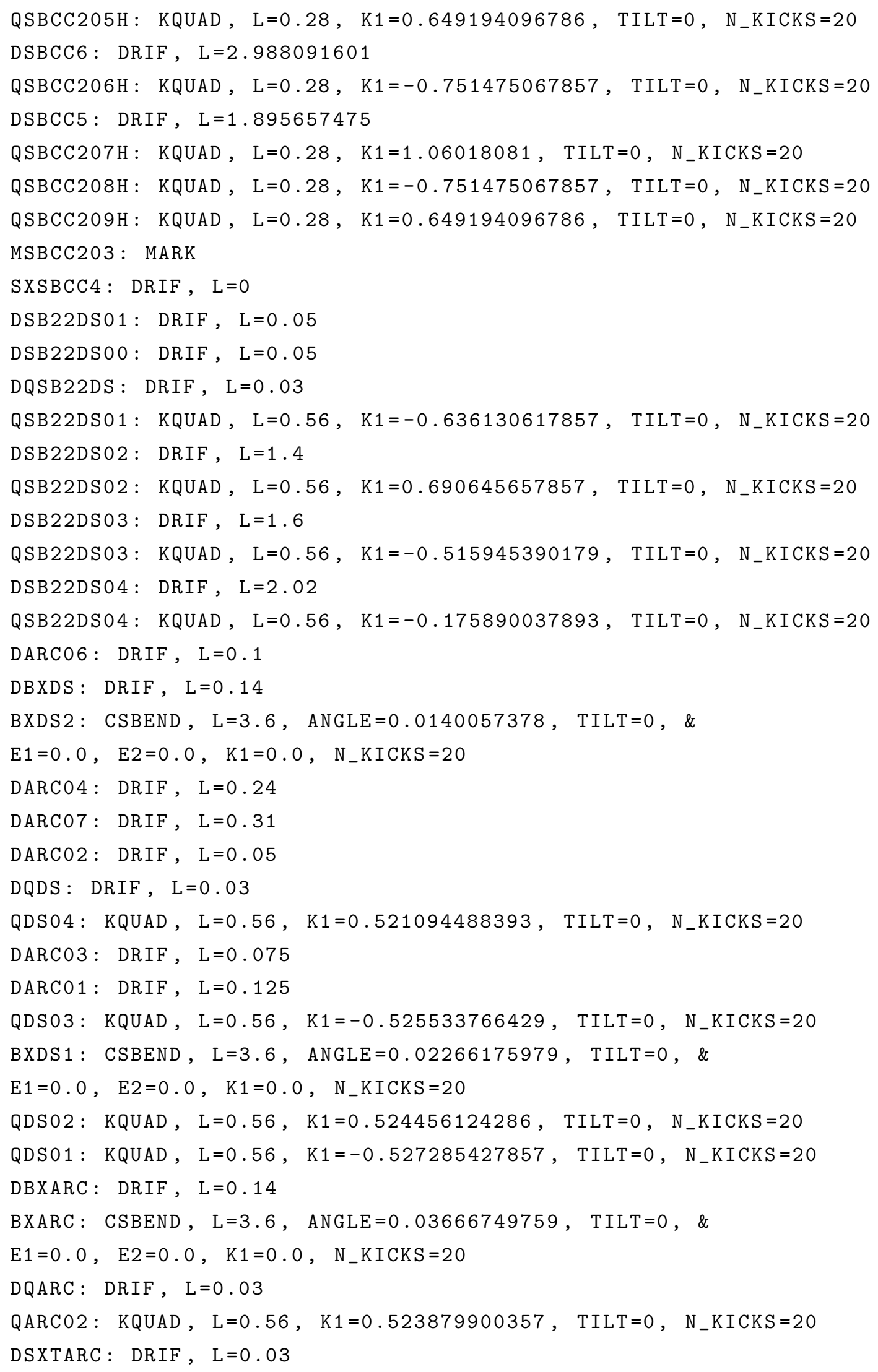




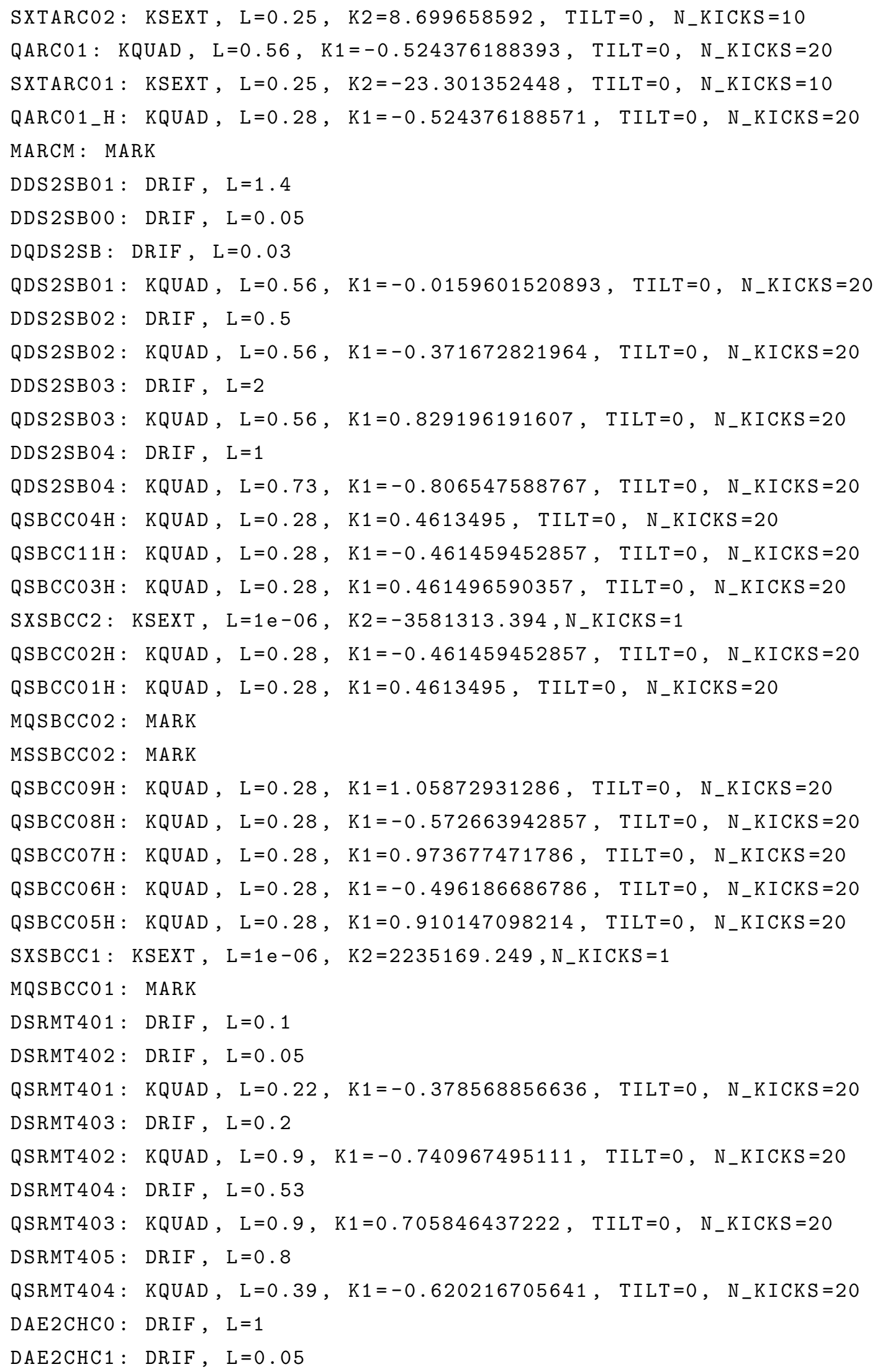




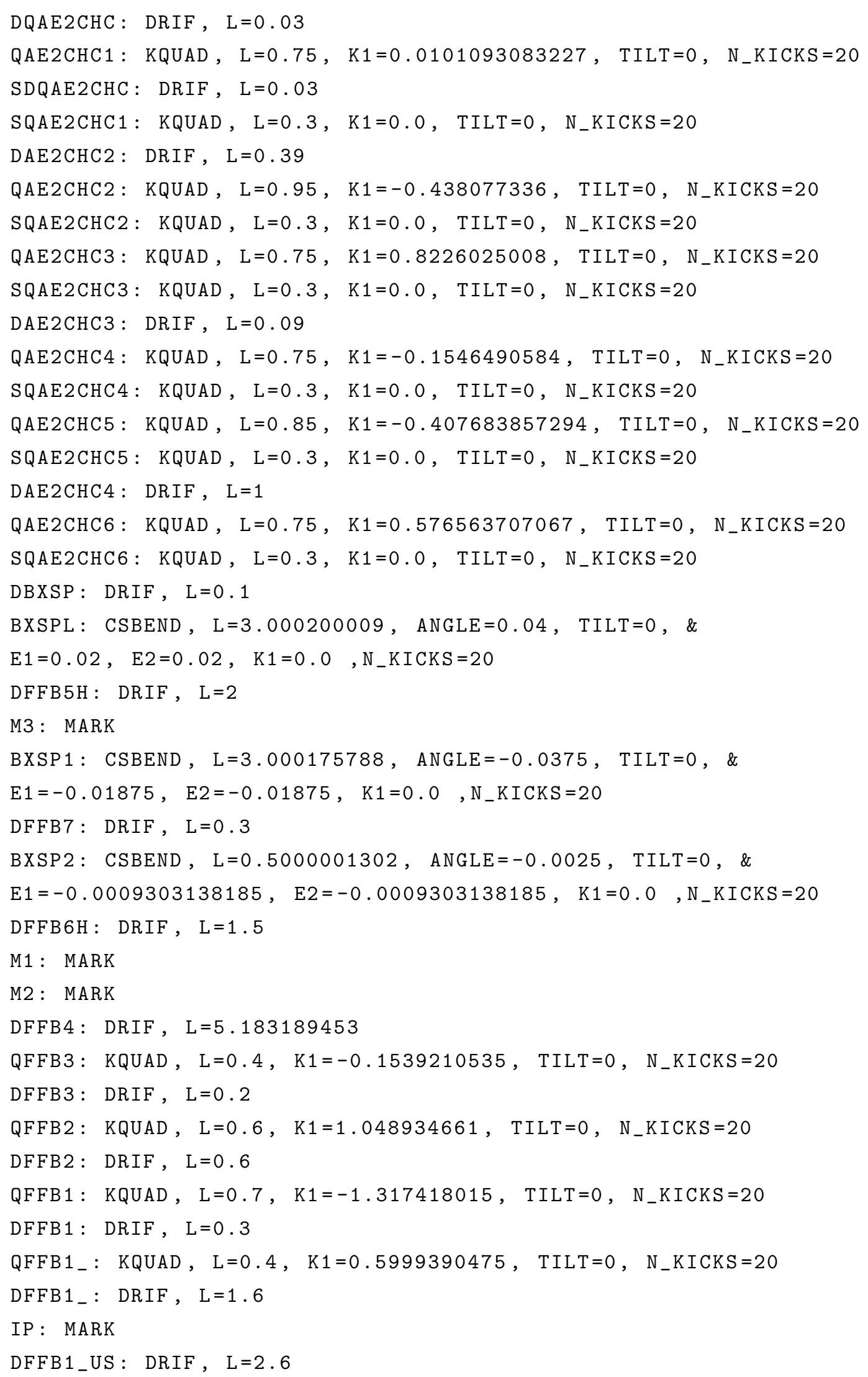




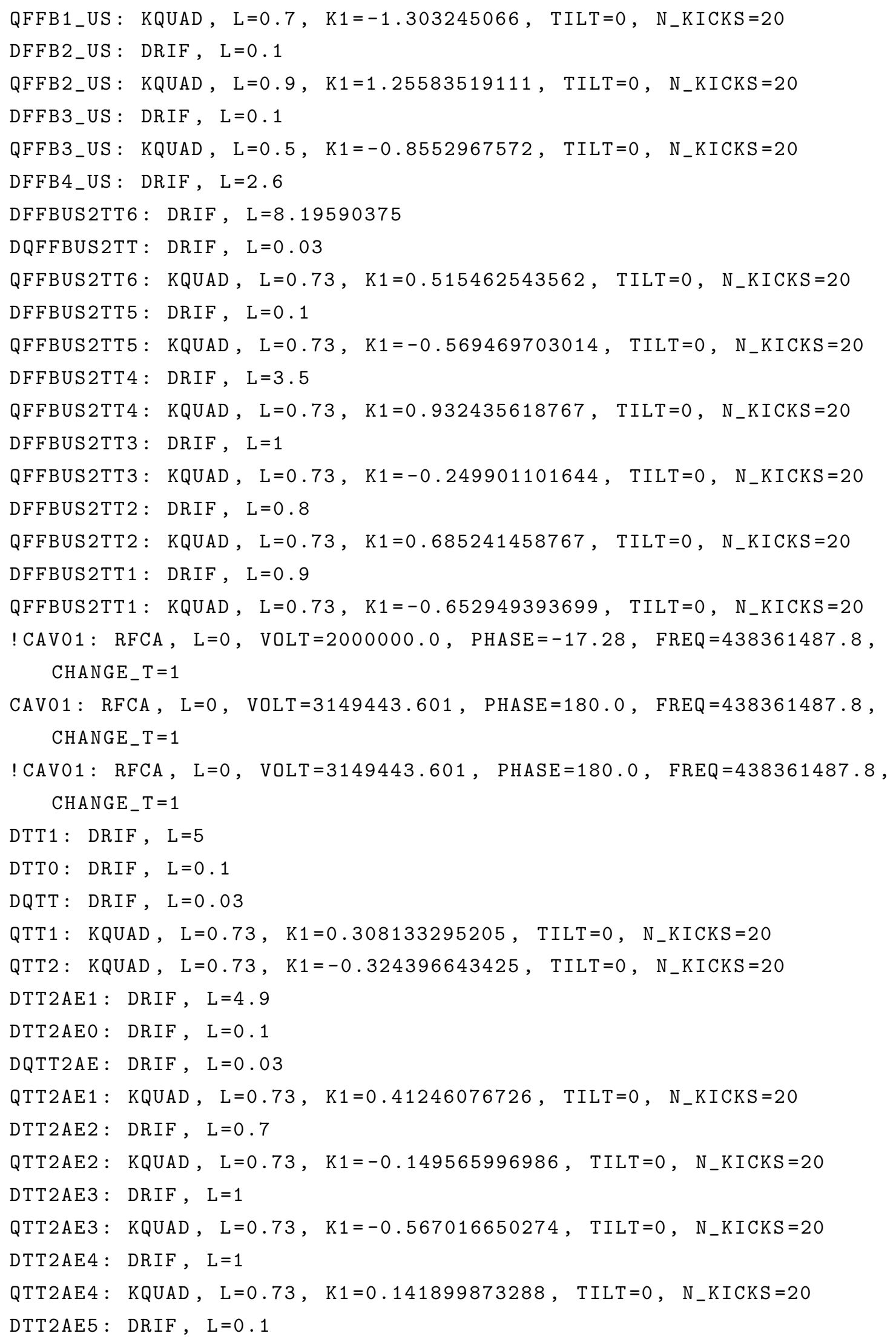




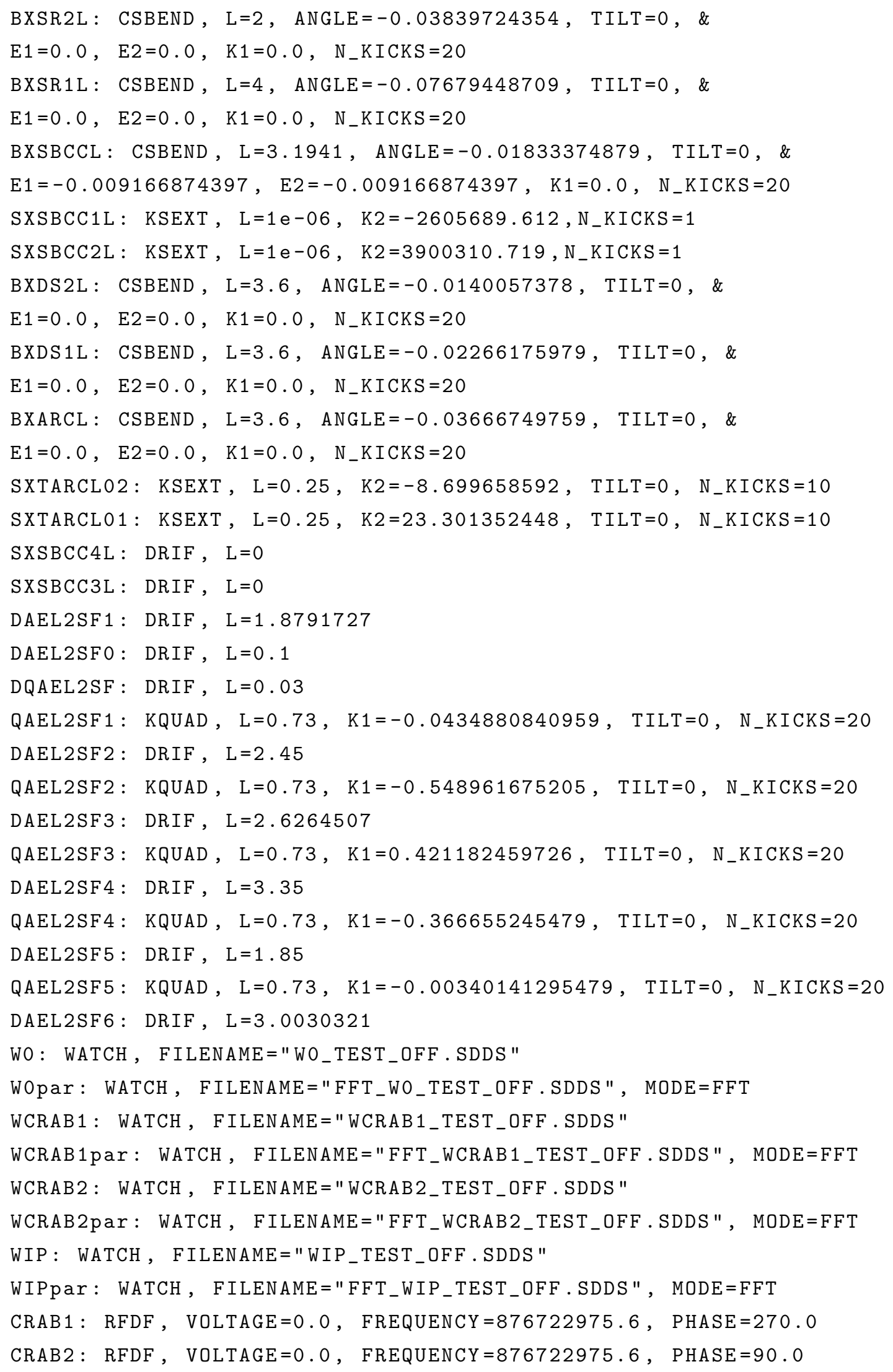


CR4: LINE $=(\&$

WO, WOpar, QSF1_US_H , DQSF_US, DSF1_US , BXYC, DSFO_US , BPM, DSFO_US, DQSF_US , QSF2_US, DQSF_US , \&

DSF1_US , BXYC , DSFO_US , BPM , DSFO_US , DQSF_US, QSF1_US, DQSF_US , DSF1_US , BXYC , \& DSF0_US, BPM , DSFO_US, DQSF_US, QSF2_US, DQSF_US , DSF1_US, BXYC, DSFO_US , BPM , \& DSFO_US, DQSF_US, QSF1_US, DQSF_US , DSF1_US , BXYC , DSFO_US , BPM , DSFO_US , DQSF_US , \&

QSF2_US , DQSF_US, DSF1_US , BXYC , DSFO_US , BPM , DSF0_US , DQSF_US , QSF1_US , DQSF_US , \& DSF1_US , BXYC , DSFO_US , BPM , DSF0_US, DQSF_US , QSF2_US , DQSF_US , DSF1_US , BXYC , \& DSFO_US, BPM , DSFO_US, DQSF_US, QSF1_US, DQSF_US , DSF1_US , BXYC , DSFO_US , BPM , \& DSFO_US, DQSF_US, QSF2_US, DQSF_US , DSF1_US , BXYC , DSFO_US , BPM , DSFO_US , DQSF_US , \& QSF1_US, DQSF_US, DSF1_US, BXYC, DSFO_US, BPM , DSF0_US , DQSF_US, QSF2_US , DQSF_US , \& DSF2AE1, BXYC, DSF2AE0, BPM, DSF2AE0, DQSF2AE, QSF2AE1, DQSF2AE, DSF2AE2, BXYC , \& DSF2AE0, BPM , DSF2AE0, DQSF2AE, QSF2AE2, DQSF2AE, DSF2AE3, BXYC, DSF2AE0, BPM , \& DSF2AE0, DQSF2AE, QSF2AE3, DQSF2AE, DSF2AE4, BXYC, DSF2AE0, BPM, DSF2AE0, DQSF $2 A E$, \& QSF2AE4, DQSF2AE, DSF2AE5, DSRM301, BPM, DSRM302, BXYC, DSRM302, DQSRM3, QSRM304 , \&

DQSRM3 , DSRM303, DBXSR , BXSR2, DBXSR , DSRM304, BPM, DSRM302, BXYC , DSRM302, \& DQSRM3 , QSRM305, DQSRM3 , DSRM304, DQSRM3 , QSRM306_H , MSRMT3 , QSRM306_H , DQSRM3 , DSRM30 4 , \&

DQSRM3 , QSRM305 , DQSRM3 , DSRM302, BXYC , DSRM302 , BPM , DSRM304, DBXSR , BXSR2 , \& DBXSR , DSRM303, DQSRM3 , QSRM304, DQSRM3 , DSRM302, BXYC , DSRM302, BPM , DSRM301, \& SOLDRIF02, DSRDC01, BPM, DSRDC02, BXYC, DSRDC03 , DQSRDC , QSRDC01, DQSRDC , DSRDC0 4 , \&

DQSRDC , QSRDC02, DQSRDC , DSRDC0 5 , BPM , DSRDC02 , BXYC , DSRDC03 , DQSRDC , QSRDC0 3 , \& DQSRDC , DSRDC03, BPM , DSRDC02, BXYC , DSRDC05, DQSRDC, QSRDC02, DQSRDC, DSRDC0 4 \& DQSRDC , QSRDC01, DQSRDC , DSRDC03 , BPM , DSRDC02, BXYC , DSRDC01, SOLDRIF02 , DSRM201, \&

BPM , DSRM202, BXYC , DSRM202, DQSRM2 , QSRM205, DQSRM2 , DSRM203, BPM , DSRM202 , \& BXYC , DSRM202, DQSRM2, QSRM206, DQSRM2, DSRM203, DBXSR, BXSR1, DBXSR, DSRM204, \& BPM , DSRM202 , BXYC , DSRM202, DQSRM2 , QSRM207, DQSRM2 , DSRM204, DQSRM2, QSRM208_H , \&

MSRMT2, QSRM208_H , DQSRM2 , DSRM204,DQSRM2, QSRM207, DQSRM2 , DSRM202, BXYC , DSRM202, \&

BPM , DSRM204, DBXSR , BXSR1, DBXSR , DSRM203, DQSRM2, QSRM206, DQSRM2 , DSRM202 , \& BXYC , DSRM202, BPM , DSRM203, DQSRM2, QSRM205, DQSRM2 , DSRM202, BXYC , DSRM202 , \& BPM , DSRM201, SOLDRIF01, DSRDC01, BPM , DSRDC02, BXYC , DSRDC03, DQSRDC , QSRDC01, \& 
DQSRDC , DSRDC04, DQSRDC , QSRDC02, DQSRDC , DSRDC05, BPM , DSRDC02, BXYC , DSRDC03 , \& DQSRDC , QSRDC03, DQSRDC , DSRDC03 , BPM , DSRDC02 , BXYC , DSRDC0 5 , DQSRDC , QSRDC02 , \& DQSRDC , DSRDC04, DQSRDC , QSRDC01, DQSRDC , DSRDC03, BPM , DSRDC02, BXYC, DSRDC01, \& SOLDRIF01, DSRMT07, DQSRM, QSRMT08, DQSRM, DSRMT07, BPM , DSRMT07 , BXYC , DSRMT10

, \&

DSRMT07, DQSRM , QSRMT07, DQSRM , DSRMT07, BPM , DSRMT07, BXYC , DSRMT09 , DSRMT07 , \& DQSRM , QSRMT06 , DQSRM , DSRMT07 , BPM , DSRMT07 , BXYC , DSRMT08 , DSRMT07 , DQSRM , \& QSRMT05 , DQSRM , DSRMT07 , BPM , DSRMT07 , BXYC , DSRMT06 , DQSBCC , QSBCC204H , QSBCC204H , \& DQSBCC, MSBCC 202 , DSBCC 1 , DBXSBCC, BXSBCC, DBXSBCC, DSBCC 1, DQSBCC, QSBCC $211 \mathrm{H}$, QSBCC $211 \mathrm{H}, \&$

DQSBCC , DSBCC4, DQSBCC, QSBCC203H, QSBCC203H, DQSBCC , SXSBCC3, DSBCC3, DQSBCC, QSBCC $202 \mathrm{H}, \&$

QSBCC 202H, DQSBCC, DSBCC1, DBXSBCC, BXSBCC, DBXSBCC, DSBCC1, DQSBCC, QSBCC201H, MQSBCC 201 , \&

QSBCC201H, DQSBCC , DSBCC1, DBXSBCC , BXSBCC , DBXSBCC , DSBCC1, DQSBCC, QSBCC202H , QSBCC $202 \mathrm{H}$, \&

DQSBCC , DSBCC3, DQSBCC, QSBCC 203H , QSBCC203H, DQSBCC , SXSBCC3, DSBCC4, DQSBCC, QSBCC $211 \mathrm{H}, \&$

QSBCC 211H, DQSBCC , DSBCC1, DBXSBCC , BXSBCC, DBXSBCC, DSBCC1, DQSBCC , QSBCC205H , QSBCC $205 \mathrm{H}, \&$

DQSBCC , DSBCC6, DQSBCC , QSBCC206H, QSBCC206H, DQSBCC, DSBCC5, DQSBCC , QSBCC 207H , QSBCC207H , \&

DQSBCC , DSBCC5, DQSBCC , QSBCC 208H, QSBCC208H, DQSBCC , DSBCC6, DQSBCC , QSBCC209H , QSBCC209H , \&

DQSBCC, MSBCC203, DSBCC 1, DBXSBCC, BXSBCC, DBXSBCC, DSBCC1, DQSBCC, QSBCC211H, QSBCC $211 \mathrm{H}, \&$

DQSBCC , SXSBCC4, DSBCC4, DQSBCC , QSBCC203H, QSBCC203H, DQSBCC, DSBCC3, DQSBCC , QSBCC $202 \mathrm{H}, \&$

QSBCC 202H, DQSBCC, DSBCC1, DBXSBCC, BXSBCC, DBXSBCC, DSBCC1, DQSBCC, QSBCC 201H, QSBCC $201 \mathrm{H}, \&$

DQSBCC , DSBCC1, DBXSBCC, BXSBCC, DBXSBCC, DSBCC1, DQSBCC, QSBCC202H, QSBCC202H, DQSBCC , \&

SXSBCC4, DSBCC3, DQSBCC, QSBCC203H, QSBCC203H , DQSBCC , DSBCC4, DQSBCC , QSBCC $211 \mathrm{H}, \mathrm{QSBCC} 211 \mathrm{H}$, \&

DQSBCC , DSBCC1, DBXSBCC, BXSBCC, DBXSBCC, DSBCC1, DQSBCC, QSBCC 204H, QSBCC204H, DQSBCC , \&

DSB22DS01, BXYC, DSB22DS00, BPM , DSB22DS00, DQSB22DS, QSB22DS01, DQSB22DS , DSB22DSO0, DSB22DSO2, \&

BXYC , DSB22DS00, BPM, DSB22DS00, DQSB22DS, QSB22DSO2, DQSB22DS, DSB22DS00 , DSB22DSO3, BXYC , \& 
DSB22DS00, BPM, DSB22DS00, DQSB22DS, QSB22DS03, DQSB22DS, DSB22DS00, DSB22DS0 4 , BXYC , DSB22DSO0, \&

BPM , DSB22DS00, DQSB22DS , QSB22DS04, DQSB22DS , DSB22DS00, DARC06, DBXDS , BXDS2 , DBXDS , \&

DARC04, DARC07, DARC02, DQDS , QDS04, DQDS , DARC03, BPM , DARC02, BXYC , \& DARC01, DBXDS , BXDS2 , DBXDS , DARC04, DARC07, DARC02, DQDS , QDS03 , DQDS , \& DARC03 , BPM , DARC02, BXYC, DARC01, DBXDS , BXDS1, DBXDS , DARC04, DARC07, \& DARC02 , DQDS , QDS02 , DQDS , DARC03 , BPM , DARC02, BXYC , DARC01, DBXDS , \& BXDS1, DBXDS , DARC04, DARC07, DARC02, DQDS , QDS01, DQDS , DARC03 , BPM , \& DARC02, BXYC , DARC01, DBXARC, BXARC , DBXARC , DARC01, BXYC , DARC02, BPM , \& DARC03, DQARC, QARC02, DQARC, DARC02, DSXTARC , SXTARC02, DSXTARC, DARC04, DBXARC , \&

BXARC, DBXARC, DARC01, BXYC, DARC02, BPM , DARC03, DQARC, QARC01, DQARC , \& DARC02, DSXTARC, SXTARC01, DSXTARC, DARC04, DBXARC, BXARC, DBXARC, DARC01, BXYC , \& DARC02, BPM , DARC03, DQARC, QARC02, DQARC, DARC02, DSXTARC, SXTARC02, DSXTARC , \& DARC04, DBXARC, BXARC, DBXARC, DARC01, BXYC, DARC02, BPM , DARC03, DQARC , \& QARC01, DQARC, DARC02, DSXTARC, SXTARC01, DSXTARC, DARC04, DBXARC, BXARC, DBXARC , \&

DARC01, BXYC , DARC02, BPM , DARC03, DQARC, QARC02, DQARC, DARC02, DSXTARC , \& SXTARCO2, DSXTARC, DARC04, DBXARC, BXARC, DBXARC, DARC01, BXYC , DARC02, BPM , \& DARC03, DQARC, QARC01, DQARC, DARC02, DSXTARC, SXTARC01, DSXTARC, DARC04, DBXARC , \&

BXARC, DBXARC, DARC01, BXYC, DARC02, BPM , DARC03, DQARC, QARC02, DQARC , \& DARC02, DSXTARC, SXTARC02, DSXTARC, DARC04, DBXARC, BXARC, DBXARC, DARC01, BXYC , \&

DARC02, BPM , DARC03, DQARC, QARC01, DQARC, DARC02, DSXTARC, SXTARC01, DSXTARC , \& DARC04, DBXARC, BXARC, DBXARC, DARC01, BXYC, DARC02, BPM , DARC03, DQARC , \& QARC02, DQARC, DARC02, DSXTARC, SXTARC02, DSXTARC, DARC04, DBXARC, BXARC, DBXARC , \&

DARC01, BXYC, DARC02, BPM , DARC03, DQARC, QARC01, DQARC, DARC02, DSXTARC , \& SXTARC01, DSXTARC, DARC04, DBXARC, BXARC, DBXARC, DARC01, BXYC, DARC02, BPM , \& DARC03, DQARC, QARC02, DQARC, DARC02, DSXTARC, SXTARC02, DSXTARC, DARC04, DBXARC , \&

BXARC, DBXARC , DARC01, BXYC, DARC02, BPM , DARC03, DQARC , QARC01, DQARC , \& DARC02, DSXTARC, SXTARC01, DSXTARC, DARC04, DBXARC, BXARC, DBXARC, DARC01, BXYC , \&

DARC02, BPM , DARC03, DQARC, QARC02, DQARC, DARC02, DSXTARC, SXTARC02, DSXTARC \& DARC04, DBXARC, BXARC, DBXARC , DARC01, BXYC , DARC02, BPM , DARC03, DQARC , \& QARC01, DQARC, DARC02, DSXTARC, SXTARC01, DSXTARC, DARC04, DBXARC, BXARC, DBXARC , \& 
DARC01, BXYC, DARC02, BPM, DARC03, DQARC, QARC02, DQARC, DARC02, DSXTARC, \& SXTARC02, DSXTARC , DARC04, DBXARC, BXARC , DBXARC , DARC01, BXYC , DARC02, BPM , \& DARC03, DQARC , QARC01, DQARC, DARC02, DSXTARC , SXTARC01, DSXTARC, DARC04, DBXARC , \&

BXARC, DBXARC, DARC01, BXYC, DARC02, BPM, DARC03, DQARC , QARC02, DQARC , \& DARC02, DSXTARC, SXTARC02, DSXTARC, DARC04, DBXARC, BXARC, DBXARC, DARC01, BXYC , \& DARC02, BPM , DARC03, DQARC, QARC01, DQARC, DARC02, DSXTARC , SXTARC01, DSXTARC , \& DARC0 4, DBXARC , BXARC , DBXARC , DARC01, BXYC , DARC02, BPM , DARC03, DQARC , \& QARCO2, DQARC, DARC02, DSXTARC, SXTARC02, DSXTARC, DARC04, DBXARC, BXARC, DBXARC , \& DARC01, BXYC , DARC02, BPM , DARC03, DQARC, QARC01, DQARC, DARC02, DSXTARC , \& SXTARC01, DSXTARC, DARC04, DBXARC , BXARC, DBXARC, DARC01, BXYC, DARC02, BPM , \& DARC03, DQARC , QARC02, DQARC, DARC02, DSXTARC , SXTARC02, DSXTARC , DARC04, DBXARC , \& BXARC, DBXARC, DARC01, BXYC, DARC02, BPM , DARC03, DQARC, QARC01, DQARC , \& DARC02, DSXTARC, SXTARC01, DSXTARC, DARC04, DBXARC, BXARC, DBXARC, DARC01, BXYC , \&

DARC02, BPM , DARC03, DQARC, QARC02, DQARC, DARC02, DSXTARC, SXTARC02, DSXTARC \& DARC04, DBXARC, BXARC, DBXARC, DARC01, BXYC, DARC02, BPM , DARC03, DQARC , \& QARC01, DQARC, DARC02, DSXTARC, SXTARC01, DSXTARC, DARC04, DBXARC, BXARC, DBXARC , \&

DARC01, BXYC, DARC02, BPM , DARC03, DQARC, QARC02, DQARC, DARC02, DSXTARC , \& SXTARCO2, DSXTARC, DARC04, DBXARC, BXARC, DBXARC, DARC01, BXYC, DARC02, BPM , \& DARC03, DQARC, QARC01, DQARC, DARC02, DSXTARC, SXTARC01, DSXTARC, DARC04, DBXARC , \&

BXARC, DBXARC, DARC01, BXYC, DARC02, BPM, DARC03, DQARC, QARC02, DQARC , \& DARC02, DSXTARC, SXTARC02, DSXTARC, DARC04, DBXARC, BXARC, DBXARC, DARC01, BXYC , \&

DARC02, BPM , DARC03, DQARC, QARC01, DQARC, DARC02, DSXTARC, SXTARC01, DSXTARC , \& DARC0 4 , DBXARC , BXARC , DBXARC , DARC01, BXYC , DARCO2, BPM , DARC03, DQARC , \& QARC02, DQARC, DARC02, DSXTARC, SXTARC02, DSXTARC, DARC04, DBXARC, BXARC, DBXARC , \&

DARC01, BXYC , DARC02, BPM , DARC03, DQARC, QARC01, DQARC , DARC02, DSXTARC , \& SXTARC01, DSXTARC, DARC04, DBXARC, BXARC, DBXARC, DARC01, BXYC, DARC02, BPM , \& DARC03, DQARC, QARC02, DQARC, DARC02, DSXTARC, SXTARC02, DSXTARC, DARC04, DBXARC , \&

BXARC, DBXARC, DARC01, BXYC, DARC02, BPM, DARC03, DQARC, QARC01, DQARC , \& DARC02, DSXTARC, SXTARC01, DSXTARC, DARC04, DBXARC, BXARC, DBXARC, DARC01, BXYC , \&

DARC02, BPM , DARC03, DQARC, QARC02, DQARC, DARC02, DSXTARC, SXTARC02, DSXTARC , \& 
DARC04, DBXARC, BXARC, DBXARC , DARC01, BXYC , DARC02, BPM, DARC03, DQARC , \& QARC01, DQARC, DARC02, DSXTARC, SXTARC01, DSXTARC, DARC04, DBXARC, BXARC, DBXARC , \& DARC01, BXYC , DARC02, BPM , DARC03, DQARC, QARC02, DQARC , DARC02, DSXTARC , \& SXTARC02, DSXTARC, DARC04, DBXARC, BXARC, DBXARC, DARC01, BXYC, DARC02, BPM , \& DARC03, DQARC, QARC01, DQARC, DARC02, DSXTARC , SXTARC01, DSXTARC, DARC04, DBXARC , \& BXARC, DBXARC , DARC01, BXYC , DARC02, BPM , DARC03 , DQARC , QARC02, DQARC , \& DARC02, DSXTARC, SXTARC02, DSXTARC, DARC04, DBXARC, BXARC, DBXARC, DARC01, BXYC , \&

DARC02, BPM , DARC03, DQARC, QARC01, DQARC, DARC02, DSXTARC , SXTARC01, DSXTARC \& DARC0 4, DBXARC , BXARC , DBXARC , DARC01, BXYC , DARC02 , BPM , DARC03, DQARC , \& QARC02, DQARC, DARC02, DSXTARC, SXTARC02, DSXTARC, DARC04, DBXARC, BXARC, DBXARC , \&

DARC01, BXYC , DARC02, BPM , DARC03, DQARC, QARC01, DQARC, DARC02, DSXTARC , \& SXTARC01, DSXTARC, DARC04, DBXARC, BXARC, DBXARC, DARC01, BXYC, DARC02, BPM , \& DARC03, DQARC , QARC02, DQARC , DARC02, DARC07, DARC04, DBXARC , BXARC , DBXARC , \& DARC01, BXYC , DARC02, BPM , DARC03, DQARC, QARC01, DQARC , DARC02, DARC07 , \& DARC0 4, DBXARC , BXARC , DBXARC , DARC01, BXYC , DARC02, BPM , DARC03, DQARC , \& QARC02, DQARC, DARC02, DARC07, DARC04, DBXARC, BXARC, DBXARC, DARC01, BXYC , \& DARC02 , BPM , DARC03, DQARC , QARC01, DQARC , DARC02, DARC07, DARC04, DBXARC , \& BXARC, DBXARC, DARC01, BXYC, DARC02, BPM , DARC03, DQARC, QARC02, DQARC , \& DARC02, DARC07, DARC04, DBXARC, BXARC, DBXARC , DARC01, BXYC, DARC02, BPM , \& DARC03, DQARC, QARC01, DQARC, DARC02, DARC07, DARC04, DBXARC, BXARC, DBXARC , \& DARC01, BXYC , DARC02 , BPM , DARC03 , DQARC , QARC02, DQARC , DARC02, DARC07 , \& DARC04, DBXARC, BXARC, DBXARC, DARC01, BXYC, DARC02, BPM , DARC03, DQARC , \& QARC01, DQARC , DARC02, DARC07, DARC04, DBXARC , BXARC, DBXARC , DARC01, BXYC , \& DARC02, BPM , DARC03, DQARC, QARC02, DQARC, DARC02, DARC07, DARC04, DBXARC , \& BXARC, DBXARC , DARC01, BXYC , DARC02 , BPM , DARC03, DQARC , QARC01_H , MARCM , \& QARC01_H, DQARC, DARC02, DARC07, DARC04, DBXARC, BXARC, DBXARC, DARC01, BXYC , \& DARC02, BPM , DARC03, DQARC, QARC02, DQARC, DARC02, DARC07, DARC04, DBXARC , \& BXARC, DBXARC, DARC01, BXYC, DARC02, BPM , DARC03, DQARC , QARC01, DQARC , \& DARC02, DARC07, DARC04, DBXARC, BXARC , DBXARC , DARC01, BXYC , DARC02, BPM , \& DARC03, DQARC, QARC02, DQARC, DARC02, DARC07, DARC04, DBXARC, BXARC, DBXARC , \& DARC01, BXYC , DARC02, BPM , DARC03 , DQARC , QARC01, DQARC , DARC02, DARC07 , \& DARC0 4, DBXARC, BXARC, DBXARC, DARC01, BXYC, DARC02, BPM, DARC03, DQARC , \& QARC02, DQARC, DARC02, DARC07, DARC04, DBXARC, BXARC, DBXARC, DARC01, BXYC , \& DARC02, BPM , DARC03, DQARC, QARC01, DQARC, DARC02, DARC07, DARC04, DBXARC , \& BXARC, DBXARC, DARC01, BXYC, DARC02, BPM , DARC03, DQARC , QARC02, DQARC , \& DARC02, DARC07, DARC04, DBXARC, BXARC, DBXARC, DARC01, BXYC , DARC02, BPM , \& DARC03, DQARC , QARC01, DQARC , DARC02, DARC07, DARC04, DBXARC , BXARC , DBXARC , \& 
DARC01, BXYC , DARC02, BPM , DARC03, DQARC, QARC02, DQARC , DARC02, DSXTARC , \& SXTARC02, DSXTARC , DARC04, DBXARC, BXARC , DBXARC , DARC01, BXYC , DARC02, BPM , \& DARC03, DQARC , QARC01, DQARC, DARC02, DSXTARC , SXTARC01, DSXTARC, DARC04, DBXARC , \&

BXARC, DBXARC , DARC01, BXYC, DARC02, BPM , DARC03, DQARC , QARC02, DQARC , \& DARC02, DSXTARC, SXTARC02, DSXTARC, DARC04, DBXARC, BXARC, DBXARC, DARC01, BXYC , \& DARC02, BPM , DARC03, DQARC, QARC01, DQARC, DARC02, DSXTARC , SXTARC01, DSXTARC , \& DARC0 4, DBXARC , BXARC , DBXARC , DARC01, BXYC , DARC02, BPM , DARC03, DQARC , \& QARCO2, DQARC, DARC02, DSXTARC, SXTARC02, DSXTARC, DARC04, DBXARC, BXARC, DBXARC , \& DARC01, BXYC , DARC02, BPM , DARC03, DQARC , QARC01, DQARC , DARC02, DSXTARC , \& SXTARC01, DSXTARC, DARC04, DBXARC , BXARC, DBXARC, DARC01, BXYC, DARC02, BPM , \& DARC03, DQARC , QARC02, DQARC, DARC02, DSXTARC , SXTARC02, DSXTARC , DARC04, DBXARC , \& BXARC, DBXARC, DARC01, BXYC, DARC02, BPM , DARC03, DQARC, QARC01, DQARC , \& DARC02, DSXTARC, SXTARC01, DSXTARC, DARC04, DBXARC, BXARC, DBXARC, DARC01, BXYC , \&

DARC02, BPM , DARC03, DQARC, QARC02, DQARC, DARC02, DSXTARC, SXTARC02, DSXTARC \& DARC04, DBXARC, BXARC, DBXARC, DARC01, BXYC, DARC02, BPM , DARC03, DQARC , \& QARC01, DQARC, DARC02, DSXTARC, SXTARC01, DSXTARC, DARC04, DBXARC, BXARC, DBXARC , \&

DARC01, BXYC, DARC02, BPM , DARC03, DQARC, QARC02, DQARC, DARC02, DSXTARC , \& SXTARCO2, DSXTARC, DARC04, DBXARC, BXARC, DBXARC, DARC01, BXYC, DARC02, BPM , \& DARC03, DQARC, QARC01, DQARC, DARC02, DSXTARC, SXTARC01, DSXTARC, DARC04, DBXARC , \&

BXARC, DBXARC, DARC01, BXYC, DARC02, BPM, DARC03, DQARC, QARC02, DQARC , \& DARC02, DSXTARC, SXTARC02, DSXTARC, DARC04, DBXARC, BXARC, DBXARC, DARC01, BXYC , \&

DARC02, BPM , DARC03, DQARC, QARC01, DQARC, DARC02, DSXTARC, SXTARC01, DSXTARC , \& DARCO 4 , DBXARC , BXARC , DBXARC , DARC01, BXYC , DARCO2, BPM , DARC03, DQARC , \& QARC02, DQARC, DARC02, DSXTARC, SXTARC02, DSXTARC, DARC04, DBXARC, BXARC, DBXARC , \&

DARC01, BXYC , DARC02, BPM , DARC03, DQARC, QARC01, DQARC, DARC02, DSXTARC , \& SXTARC01, DSXTARC, DARC04, DBXARC, BXARC, DBXARC, DARC01, BXYC, DARC02, BPM , \& DARC03, DQARC, QARC02, DQARC, DARC02, DSXTARC, SXTARC02, DSXTARC, DARC04, DBXARC , \&

BXARC, DBXARC, DARC01, BXYC, DARC02, BPM, DARC03, DQARC, QARC01, DQARC , \& DARC02, DSXTARC, SXTARC01, DSXTARC, DARC04, DBXARC, BXARC, DBXARC, DARC01, BXYC , \&

DARC02, BPM , DARC03, DQARC, QARC02, DQARC, DARC02, DSXTARC, SXTARC02, DSXTARC , \& 
DARC0 4, DBXARC, BXARC, DBXARC, DARC01, BXYC, DARC02, BPM, DARC03, DQARC , \& QARC01, DQARC, DARC02, DSXTARC, SXTARC01, DSXTARC, DARC04, DBXARC, BXARC, DBXARC , \&

DARC01, BXYC , DARC02, BPM , DARC03, DQARC, QARC02, DQARC , DARC02, DSXTARC , \& SXTARC02, DSXTARC, DARC04, DBXARC, BXARC, DBXARC, DARC01, BXYC, DARC02, BPM , \& DARC03, DQARC, QARC01, DQARC, DARC02, DSXTARC , SXTARC01, DSXTARC, DARC04, DBXARC , \& BXARC, DBXARC , DARC01, BXYC, DARC02, BPM , DARC03, DQARC , QARC02, DQARC , \& DARC02, DSXTARC, SXTARC02, DSXTARC, DARC04, DBXARC, BXARC, DBXARC, DARC01, BXYC , \&

DARC02, BPM , DARC03, DQARC, QARC01, DQARC, DARC02, DSXTARC , SXTARC01, DSXTARC \& DARC0 4, DBXARC , BXARC , DBXARC , DARC01, BXYC , DARC02 , BPM , DARC03, DQARC , \& QARC02, DQARC, DARC02, DSXTARC, SXTARC02, DSXTARC, DARC04, DBXARC, BXARC, DBXARC , \&

DARC01, BXYC , DARC02, BPM , DARC03, DQARC, QARC01, DQARC, DARC02, DSXTARC , \& SXTARC01, DSXTARC, DARC04, DBXARC, BXARC, DBXARC, DARC01, BXYC, DARC02, BPM , \& DARC03, DQARC, QARC02, DQARC, DARC02, DSXTARC, SXTARC02, DSXTARC, DARC04, DBXARC , \&

BXARC, DBXARC, DARC01, BXYC, DARC02, BPM, DARC03, DQARC, QARC01, DQARC , \& DARC02, DSXTARC, SXTARC01, DSXTARC, DARC04, DBXARC, BXARC, DBXARC, DARC01, BXYC , \&

DARC02, BPM , DARC03, DQARC, QARC02, DQARC, DARC02, DSXTARC, SXTARC02, DSXTARC , \& DARCO 4 , DBXARC , BXARC , DBXARC , DARC01, BXYC, DARC02, BPM , DARC03, DQARC , \& QARC01, DQARC, DARC02, DSXTARC, SXTARC01, DSXTARC, DARC04, DBXARC, BXARC, DBXARC , \&

DARC01, BXYC , DARC02, BPM , DARC03, DQARC, QARC02, DQARC , DARC02, DSXTARC , \& SXTARCO2, DSXTARC, DARC04, DBXARC, BXARC, DBXARC, DARC01, BXYC, DARCO2, BPM , \& DARC03, DQARC, QARC01, DQARC, DARC02, DSXTARC, SXTARC01, DSXTARC, DARC04, DBXARC , \&

BXARC, DBXARC, DARC01, BXYC, DARC02, BPM, DARC03, DQARC, QARC02, DQARC , \& DARC02, DSXTARC, SXTARC02, DSXTARC, DARC04, DBXARC, BXARC, DBXARC, DARC01, BXYC , \&

DARC02, BPM , DARC03, DQARC, QARC01, DQARC, DARC02, DSXTARC , SXTARC01, DSXTARC , \& DARC04, DBXARC, BXARC, DBXARC, DARC01, BXYC, DARC02, BPM , DARC03, DQARC , \& QARC02, DQARC, DARC02, DSXTARC, SXTARC02, DSXTARC, DARC04, DBXARC, BXARC, DBXARC , \&

DARC01, BXYC, DARC02, BPM , DARC03, DQARC, QARC01, DQARC, DARC02, DSXTARC , \& SXTARC01, DSXTARC, DARC04, DBXARC, BXARC, DBXARC, DARC01, BXYC, DARCO2, BPM , \& DARC03, DQARC, QARC02, DQARC, DARC02, DSXTARC, SXTARC02, DSXTARC, DARC04, DBXARC , \&

BXARC, DBXARC, DARC01, BXYC, DARC02, BPM, DARC03, DQARC, QARC01, DQARC , \& 
DARC02, DSXTARC, SXTARC01, DSXTARC, DARC04, DBXARC, BXARC, DBXARC, DARC01, BXYC , \&

DARC02, BPM , DARC03, DQARC, QARC02, DQARC, DARC02, DSXTARC, SXTARC02, DSXTARC , \& DARC0 4, DBXARC , BXARC , DBXARC , DARC01, BXYC , DARC02, BPM , DARC03, DQARC , \& QARC01, DQARC, DARC02, DSXTARC, SXTARC01, DSXTARC, DARC04, DBXARC, BXARC, DBXARC , \&

DARC01, BXYC , DARC02, BPM , DARC03, DQARC, QARC02, DQARC, DARC02, DARC07 , \& DARC04, DBXARC , BXARC , DBXARC , DARC01, BXYC , DARC02, BPM , DARC03, DQDS , \& QDS01, DQDS , DARC02 , DARC07 , DARC04 , DBXDS , BXDS1 , DBXDS , DARC01, BXYC , \& DARC02 , BPM , DARC03 , DQDS , QDS02 , DQDS , DARC02, DARC07 , DARC04 , DBXDS , \& BXDS1 , DBXDS , DARC01 , BXYC , DARC02 , BPM , DARC03 , DQDS , QDS03 , DQDS , \& DARC02 , DARC07 , DARC04, DBXDS , BXDS2 , DBXDS , DARC01, BXYC , DARC02, BPM , \& DARC03 , DQDS , QDS04, DQDS , DARC02, DARC07 , DARC04 , DBXDS , BXDS2 , DBXDS , \& DARC06, DDS2SB01, BXYC , DDS2SB00, BPM , DDS2SB00, DQDS2SB, QDS2SB01, DQDS2SB , DDS2SBOO, \&

DDS2SB02, BXYC , DDS2SB00, BPM, DDS2SB00, DQDS2SB, QDS2SB02, DQDS2SB, DDS2SB00, DDS2SBO3, \&

BXYC, DDS2SB00, BPM , DDS2SB00, DQDS2SB, QDS2SB03, DQDS2SB, DDS2SB00, DDS2SB0 4 , $B X Y C$, \&

DDS2SB00, BPM, DDS2SB00, DQDS2SB, QDS2SB04, DQDS2SB, DDS2SB00, DQSBCC, QSBCC04H , QSBCCO4H, \&

DQSBCC, DSBCC1, DBXSBCC, BXSBCC, DBXSBCC, DSBCC1, DQSBCC, QSBCC11H, QSBCC 11H, DQSBCC , \&

DSBCC4, DQSBCC, QSBCC03H, QSBCC03H, DQSBCC, DSBCC3, SXSBCC2, DQSBCC, QSBCC02H, QSBCCO2H , \&

DQSBCC, DSBCC1, DBXSBCC, BXSBCC, DBXSBCC, DSBCC1, DQSBCC, QSBCC01H, MQSBCC02, QSBCC01H , \&

DQSBCC, DSBCC1, DBXSBCC, BXSBCC, DBXSBCC, DSBCC1, DQSBCC, QSBCC02H, QSBCC02H, DQSBCC , \&

DSBCC 3, DQSBCC , QSBCC03H, QSBCC03H, DQSBCC, DSBCC4, MSSBCC02, SXSBCC2, DQSBCC, QSBCC11H , \&

QSBCC11H, DQSBCC, DSBCC1, DBXSBCC, BXSBCC, DBXSBCC, DSBCC1, DQSBCC, QSBCC09H, QSBCCO9H , \&

DQSBCC , DSBCC6, DQSBCC, QSBCC08H, QSBCC08H, DQSBCC, DSBCC5, DQSBCC, QSBCC07H , QSBCCO7H , \&

DQSBCC , DSBCC5, DQSBCC, QSBCC06H, QSBCC06H, DQSBCC, DSBCC6, DQSBCC, QSBCC05H , QSBCC05H , \&

DQSBCC, DSBCC1, DBXSBCC, BXSBCC, DBXSBCC, DSBCC1, DQSBCC, QSBCC11H, QSBCC11H, DQSBCC , \&

DSBCC4, SXSBCC1, DQSBCC, QSBCC03H, QSBCC03H, DQSBCC, DSBCC3, DQSBCC, QSBCC02H, $\mathrm{QSBCC} 02 \mathrm{H}$, \& 
DQSBCC , DSBCC1, DBXSBCC, BXSBCC, DBXSBCC, DSBCC1, DQSBCC , QSBCC01H, MQSBCC01, QSBCC01H, \&

DQSBCC, DSBCC1, DBXSBCC, BXSBCC, DBXSBCC, DSBCC1, DQSBCC, QSBCC02H, QSBCC02H, DQSBCC , \&

DSBCC3, SXSBCC1, CRAB1, WCRAB1, WCRAB1Par, DQSBCC, QSBCC03H, QSBCC03H, DQSBCC , DSBCC 4, DQSBCC , QSBCC $11 \mathrm{H}, \mathrm{QSBCC} 11 \mathrm{H}$, \&

DQSBCC, DSBCC1, DBXSBCC, BXSBCC, DBXSBCC, DSBCC1, DQSBCC, QSBCC04H, QSBCC04H, DQSBCC , \&

DSRMT401, BXYC , DSRMT402, DSRMT402, DQSRM, QSRMT401, DQSRM, DSRMT402, DSRMT403, $B X Y C, \&$

DSRMT402, BPM , DSRMT402, DQSRM, QSRMT402, DQSRM, DSRMT402, DSRMT404, BXYC , DSRMT $402, \&$

DSRMT402, DQSRM , QSRMT403, DQSRM, DSRMT402, DSRMT405, BXYC , DSRMT402, BPM , DSRMT $402, \&$

DQSRM , QSRMT404, DQSRM , DSRMT402, SOLDRIF01, DSRDC01, BXYC , DSRDC02, BPM , DSRDC0 3 , \&

DQSRDC , QSRDC01, DQSRDC , DSRDC04, DQSRDC , QSRDC02, DQSRDC , DSRDC05, BXYC , DSRDC02, \&

BPM , DSRDC03, DQSRDC , QSRDC03, DQSRDC , DSRDC03 , BXYC , DSRDC02 , BPM , DSRDC05 , \& DQSRDC , QSRDC02, DQSRDC , DSRDC04, DQSRDC , QSRDC01, DQSRDC , DSRDC03 , BXYC , DSRDCO2, \&

BPM , DSRDC01, SOLDRIF01, DSRM201, BPM , DSRM202, BXYC , DSRM202, DQSRM2 , QSRM205 , \& DQSRM2 , DSRM203, BPM , DSRM202, BXYC , DSRM202, DQSRM2, QSRM206, DQSRM2, DSRM203 , \& DBXSR , BXSR1 , DBXSR , DSRM204, BPM , DSRM202, BXYC , DSRM202, DQSRM2 , QSRM207 , \& DQSRM2 , DSRM204, DQSRM2, QSRM208_H , MSRMT2, QSRM208_H , DQSRM2 , DSRM204, DQSRM2 , QSRM207 , \&

DQSRM2 , DSRM202, BXYC , DSRM202, BPM , DSRM204, DBXSR , BXSR1, DBXSR , DSRM203 , \& DQSRM2 , QSRM206, DQSRM2, DSRM202, BXYC , DSRM202, BPM , DSRM203, DQSRM2 , QSRM205 , \& DQSRM2, DSRM202, BXYC , DSRM202, BPM , DSRM201, SOLDRIF02, DSRDC01, BXYC , DSRDC02 , \&

BPM , DSRDC0 3, DQSRDC , QSRDC01, DQSRDC, DSRDC04, DQSRDC, QSRDC02, DQSRDC, DSRDC05 \&

BXYC , DSRDC02, BPM , DSRDC0 3, DQSRDC , QSRDC03, DQSRDC , DSRDC0 3, BXYC , DSRDC0 2 \& BPM , DSRDC0 5 , DQSRDC , QSRDC02, DQSRDC , DSRDC04, DQSRDC , QSRDC01, DQSRDC , DSRDC0 3 ,$\&$

BXYC , DSRDC02, BPM , DSRDC01, SOLDRIF02, DSRM301, BPM, DSRM302, BXYC , DSRM302, \& DQSRM3 , QSRM304, DQSRM3 , DSRM303 , DBXSR , BXSR2 , DBXSR , DSRM304, BPM , DSRM302, \& BXYC , DSRM302 , DQSRM3 , QSRM305 , DQSRM3 , DSRM304 , DQSRM3 , QSRM306_H , MSRMT3 , QSRM306_H , \&

DQSRM3 , DSRM304, DQSRM3 , QSRM305, DQSRM3 , DSRM302, BXYC, DSRM302, BPM , DSRM304, \& DBXSR , BXSR2 , DBXSR , DSRM303, DQSRM3 , QSRM304, DQSRM3 , DSRM302, BXYC , DSRM302, \& 


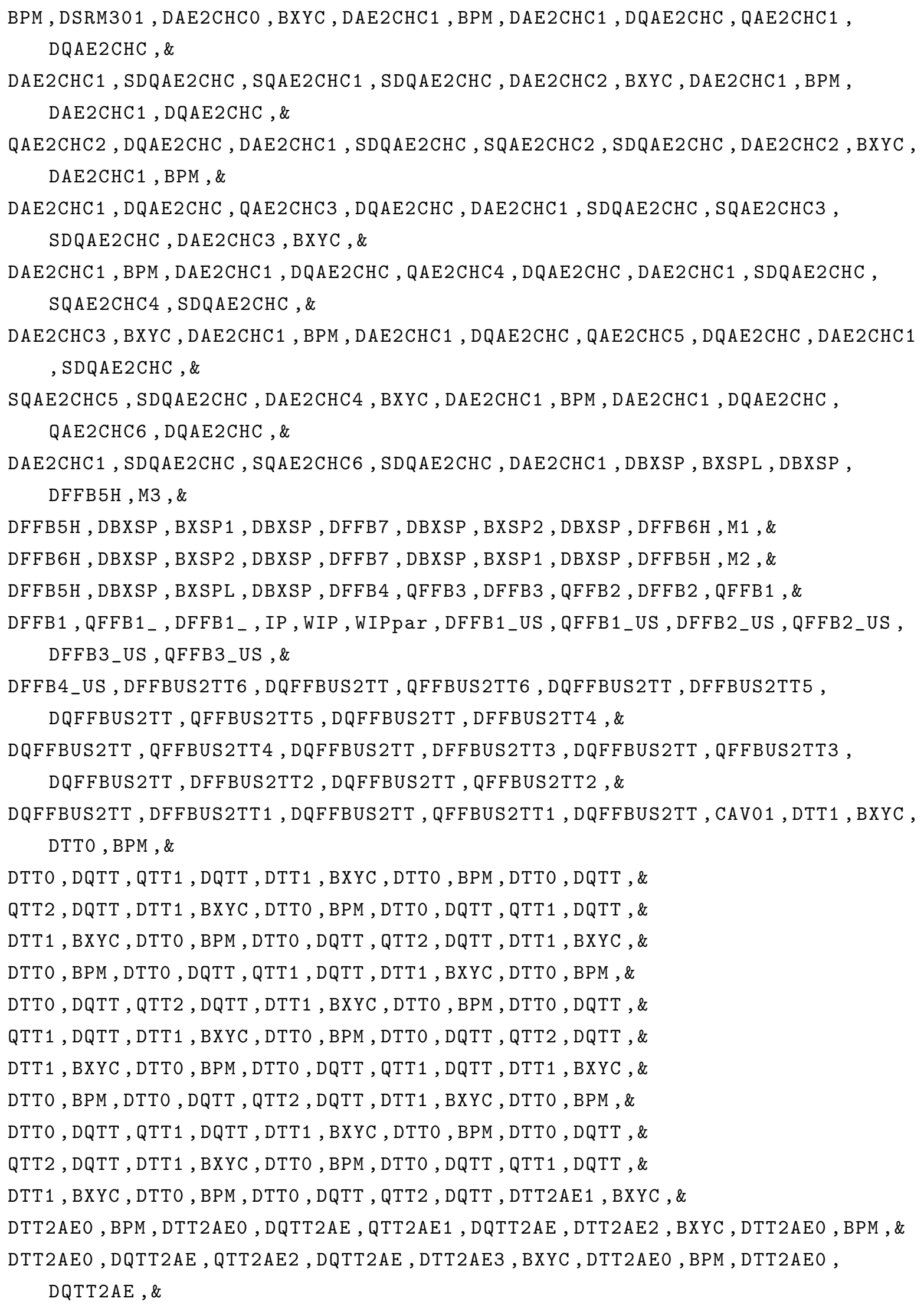


QTT2AE3, DQTT2AE, DTT2AE4, BXYC, DTT2AE0, BPM, DTT2AE0, DQTT2AE, QTT2AE4, DQTT2AE , \&

DTT2AE5 , DSRM301, BPM , DSRM302, BXYC , DSRM302, DQSRM3 , QSRM304, DQSRM3 , DSRM303 , \&

DBXSR , BXSR2L , DBXSR , DSRM304, BPM , DSRM302, BXYC , DSRM302, DQSRM3 , QSRM305 \& DQSRM3 , DSRM304, DQSRM3 , QSRM306_H , MSRMT3 , QSRM306_H , DQSRM3 , DSRM304 , DQSRM3 , QSRM305, \& DQSRM3 , DSRM302 , BXYC , DSRM302, BPM , DSRM304, DBXSR , BXSR2L , DBXSR , DSRM303 , \& DQSRM3 , QSRM304, DQSRM3 , DSRM302, BXYC , DSRM302 , BPM , DSRM301, SOLDRIF02 , DSRDC01, \&

BPM , DSRDC02, BXYC , DSRDC0 3 , DQSRDC , QSRDC01, DQSRDC , DSRDC04, DQSRDC , QSRDC02 \& DQSRDC , DSRDC05 , BPM , DSRDC02 , BXYC , DSRDC03, DQSRDC , QSRDC03, DQSRDC , DSRDC03 , \& BPM , DSRDC02, BXYC , DSRDC05, DQSRDC , QSRDC02, DQSRDC , DSRDC04, DQSRDC , QSRDC0 1 , \& DQSRDC , DSRDC0 3, BPM , DSRDC02, BXYC , DSRDC01, SOLDRIF02, DSRM201, BPM , DSRM202, \& BXYC , DSRM202, DQSRM2, QSRM205, DQSRM2, DSRM203, BPM , DSRM202, BXYC , DSRM202, \& DQSRM2, QSRM206, DQSRM2, DSRM203, DBXSR , BXSR1L , DBXSR, DSRM204, BPM , DSRM202 , \& BXYC , DSRM202, DQSRM2 , QSRM207, DQSRM2 , DSRM204,DQSRM2, QSRM208_H , MSRMT2 , QSRM208_H , \&

DQSRM2 , DSRM204, DQSRM2, QSRM207, DQSRM2, DSRM202, BXYC , DSRM202, BPM , DSRM204, \& DBXSR , BXSR1L , DBXSR, DSRM203, DQSRM2, QSRM206, DQSRM2, DSRM202, BXYC , DSRM202, \& BPM , DSRM203, DQSRM2, QSRM205, DQSRM2, DSRM202, BXYC , DSRM202, BPM , DSRM201, \& SOLDRIF01, DSRDC01, BPM , DSRDC02, BXYC , DSRDC03, DQSRDC , QSRDC01, DQSRDC , DSRDC0 4 , \&

DQSRDC , QSRDC02, DQSRDC , DSRDC05, BPM , DSRDC02, BXYC, DSRDC03, DQSRDC , QSRDC03 \& \& DQSRDC , DSRDC03, BPM , DSRDC02, BXYC , DSRDC05, DQSRDC , QSRDC02, DQSRDC , DSRDC04, \& DQSRDC , QSRDC01, DQSRDC , DSRDC03 , BPM , DSRDC02, BXYC , DSRDC01, SOLDRIF01 , DSRMT 402 , \&

DQSRM , QSRMT404, DQSRM , DSRMT402, BPM , DSRMT402, BXYC , DSRMT405, DSRMT402, DQSRM , \&

QSRMT403, DQSRM, DSRMT402, DSRMT402, BXYC, DSRMT404, DSRMT402, DQSRM, QSRMT402, DQSRM , \&

DSRMT402, BPM, DSRMT402, BXYC , DSRMT403, DSRMT402, DQSRM, QSRMT401, DQSRM , DSRMT $402, \&$

DSRMT402, BXYC, DSRMT401, DQSBCC, QSBCC04H, QSBCC04H, DQSBCC, DSBCC1, DBXSBCC , BXSBCCL , \&

DBXSBCC, DSBCC1, DQSBCC, QSBCC11H, QSBCC11H, DQSBCC, DSBCC4, DQSBCC, QSBCC03H , $\mathrm{QSBCCO} 3 \mathrm{H}, \&$

DQSBCC, CRAB2, WCRAB2, WCRAB2par, SXSBCC1L, DSBCC3, DQSBCC, QSBCC02H, QSBCC02H, DQSBCC , DSBCC 1 , DBXSBCC , BXSBCCL , \&

DBXSBCC, DSBCC1, DQSBCC, QSBCC01H, QSBCC01H, DQSBCC, DSBCC1, DBXSBCC, BXSBCCL , $\mathrm{DBXSBCC}, \&$ 
DSBCC1, DQSBCC, QSBCC02H, QSBCC02H, DQSBCC, DSBCC3, DQSBCC, QSBCC03H, QSBCC03H, DQSBCC , \&

SXSBCC1L, DSBCC4, DQSBCC, QSBCC11H, QSBCC11H, DQSBCC, DSBCC1, DBXSBCC, BXSBCCL , DBXSBCC , \&

DSBCC1, DQSBCC, QSBCC05H, QSBCC05H, DQSBCC, DSBCC6, DQSBCC, QSBCC06H, QSBCC06H , DQSBCC , \&

DSBCC5, DQSBCC, QSBCC07H, QSBCC07H , DQSBCC , DSBCC5, DQSBCC , QSBCC08H, QSBCC08H , $D Q S B C C, \&$

DSBCC6, DQSBCC , QSBCC09H, QSBCC09H, DQSBCC, DSBCC1, DBXSBCC , BXSBCCL, DBXSBCC , DSBCC 1 , \&

DQSBCC , QSBCC11H , QSBCC11H, DQSBCC , SXSBCC2L , DSBCC4, DQSBCC , QSBCC 03H , QSBCCO3H , DQSBCC , \&

DSBCC 3, DQSBCC, QSBCC02H, QSBCC02H, DQSBCC, DSBCC1, DBXSBCC, BXSBCCL, DBXSBCC , DSBCC 1 , \&

DQSBCC , QSBCC01H, QSBCC01H, DQSBCC, DSBCC1, DBXSBCC, BXSBCCL, DBXSBCC, DSBCC1, $D Q S B C C, \&$

QSBCC02H, QSBCC02H, DQSBCC , SXSBCC2L , DSBCC3, DQSBCC, QSBCC03H, QSBCC03H , DQSBCC , DSBCC4, \&

DQSBCC , QSBCC11H, QSBCC11H, DQSBCC, DSBCC1, DBXSBCC , BXSBCCL, DBXSBCC, DSBCC1, DQSBCC , \&

QSBCC04H, QSBCC04H, DQSBCC, DDS2SB00, DQDS2SB, QDS2SB04, DQDS2SB, DDS2SB00, BPM , DDS2SB00, \&

BXYC, DDS2SB04, DDS2SB00, DQDS2SB, QDS2SB03, DQDS2SB, DDS2SB00, BPM, DDS2SB00, $\mathrm{BXYC}, \&$

DDS2SB03, DDS2SB00, DQDS2SB, QDS2SB02, DQDS2SB, DDS2SB00, BPM, DDS2SB00, BXYC , DDS2SBO2, \&

DDS2SB00, DQDS2SB, QDS2SB01, DQDS2SB, DDS2SB00, BPM, DDS2SB00, BXYC, DDS2SB01, DARCO6, \&

DBXDS , BXDS2L , DBXDS , DARC04, DARC07, DARC02, DQDS , QDSO 4 , DQDS , DARC03 , \& BPM , DARC02, BXYC , DARC01, DBXDS , BXDS2L, DBXDS , DARC04, DARC07, DARC02 , \& DQDS , QDS03 , DQDS , DARC03 , BPM , DARC02, BXYC , DARC01, DBXDS , BXDS1L , \& DBXDS , DARC04 , DARC07 , DARC02, DQDS , QDS02, DQDS , DARC03 , BPM , DARC02 , \& BXYC , DARC01, DBXDS , BXDS1L , DBXDS , DARC04 , DARC07, DARC02 , DQDS , QDSO 1 , \& DQDS , DARC03 , BPM , DARC02, BXYC , DARC01, DBXARC , BXARCL , DBXARC , DARC01, \& BXYC , DARC02, BPM , DARC03, DQARC , QARC02 , DQARC , DARC02, DSXTARC , SXTARCL02, \& DSXTARC , DARC04, DBXARC , BXARCL , DBXARC , DARC01, BXYC , DARC02, BPM , DARC03 , \& DQARC, QARC01, DQARC, DARC02, DSXTARC, SXTARCL01, DSXTARC , DARC04, DBXARC, BXARCL , \& DBXARC , DARC01, BXYC , DARC02, BPM , DARC03, DQARC, QARC02, DQARC , DARC02 , \& DSXTARC, SXTARCLO2, DSXTARC, DARC04, DBXARC, BXARCL, DBXARC, DARC01, BXYC, DARCO2, \& 
BPM, DARC03, DQARC, QARC01, DQARC, DARC02, DSXTARC , SXTARCL01, DSXTARC , DARC04, \& DBXARC , BXARCL , DBXARC, DARC01, BXYC, DARC02, BPM , DARC03, DQARC, QARC02 , \& DQARC, DARC02, DSXTARC, SXTARCLO2, DSXTARC, DARC04, DBXARC, BXARCL, DBXARC , DARC0 1 , \& BXYC , DARC02, BPM , DARC03, DQARC , QARC01, DQARC, DARC02, DSXTARC , SXTARCL01, \& DSXTARC , DARC04, DBXARC, BXARCL , DBXARC , DARC01, BXYC , DARC02, BPM , DARC03 , \& DQARC, QARC02, DQARC, DARC02, DSXTARC , SXTARCL02, DSXTARC, DARC04, DBXARC , BXARCL , \&

DBXARC , DARC01, BXYC , DARC02, BPM , DARC03, DQARC, QARC01, DQARC, DARC02 , \& DSXTARC , SXTARCL01, DSXTARC, DARC04, DBXARC , BXARCL , DBXARC , DARC01, BXYC ,

DARCO2, \&

BPM , DARC03, DQARC , QARC02, DQARC, DARC02, DSXTARC , SXTARCL02, DSXTARC, DARC04, \& DBXARC , BXARCL , DBXARC, DARC01, BXYC , DARC02, BPM , DARC03 , DQARC , QARC01 , \& DQARC, DARC02, DSXTARC, SXTARCL01, DSXTARC, DARC04, DBXARC, BXARCL, DBXARC , DARC0 1 , \& BXYC , DARC02, BPM, DARC03, DQARC, QARC02, DQARC, DARC02, DSXTARC, SXTARCLO2 , \& DSXTARC , DARC04, DBXARC, BXARCL , DBXARC , DARC01, BXYC , DARC02, BPM , DARC03 , \& DQARC, QARC01, DQARC, DARC02, DSXTARC, SXTARCL01, DSXTARC , DARC04, DBXARC , BXARCL , \& DBXARC, DARC01, BXYC, DARC02, BPM , DARC03, DQARC, QARC02, DQARC, DARC02 , \& DSXTARC, SXTARCLO2, DSXTARC, DARC04, DBXARC, BXARCL, DBXARC, DARC01, BXYC, DARCO2, \&

BPM , DARC03, DQARC, QARC01, DQARC, DARC02, DSXTARC, SXTARCL01, DSXTARC, DARC04, \& DBXARC , BXARCL , DBXARC, DARC01, BXYC , DARC02, BPM , DARC03, DQARC, QARC02 , \& DQARC , DARC02, DSXTARC , SXTARCLO2, DSXTARC , DARC04, DBXARC , BXARCL , DBXARC , DARC0 1 , \& BXYC, DARC02, BPM , DARC03, DQARC , QARC01, DQARC, DARC02, DSXTARC , SXTARCL01, \& DSXTARC , DARC04, DBXARC , BXARCL , DBXARC , DARC01, BXYC , DARC02, BPM , DARC03 , \& DQARC , QARC02, DQARC , DARC02, DSXTARC , SXTARCL02, DSXTARC , DARC04, DBXARC , BXARCL , \& DBXARC , DARC01, BXYC , DARC02, BPM , DARC03, DQARC , QARC01, DQARC, DARC02 , \& DSXTARC, SXTARCL01, DSXTARC, DARC04, DBXARC, BXARCL , DBXARC, DARC01, BXYC, DARCO 2 , \& BPM , DARC03, DQARC, QARC02, DQARC, DARC02, DSXTARC, SXTARCL02, DSXTARC, DARC04, \& DBXARC , BXARCL , DBXARC , DARC01, BXYC , DARC02 , BPM , DARC03 , DQARC , QARC01 , \& DQARC, DARC02, DSXTARC, SXTARCL01, DSXTARC, DARC04, DBXARC, BXARCL , DBXARC , DARC0 1 , \& BXYC, DARC02, BPM , DARC03, DQARC, QARC02, DQARC, DARC02, DSXTARC, SXTARCL02, \& DSXTARC , DARC04, DBXARC , BXARCL , DBXARC , DARC01, BXYC , DARC02, BPM , DARC03 , \& DQARC, QARC01, DQARC, DARC02, DSXTARC, SXTARCL01, DSXTARC , DARC04, DBXARC , BXARCL , \& 
DBXARC , DARC01, BXYC , DARC02, BPM , DARC03, DQARC, QARC02, DQARC , DARC02 , \& DSXTARC, SXTARCLO2, DSXTARC, DARC04, DBXARC, BXARCL, DBXARC, DARC01, BXYC,

DARCO2, \&

BPM, DARC03, DQARC, QARC01, DQARC, DARC02, DSXTARC, SXTARCL01, DSXTARC, DARC0 4 \& DBXARC , BXARCL , DBXARC, DARC01, BXYC, DARC02, BPM, DARC03, DQARC, QARC02, \& DQARC, DARC02, DSXTARC, SXTARCLO2, DSXTARC, DARCO4, DBXARC, BXARCL, DBXARC , DARC0 1 , \& BXYC , DARC02, BPM , DARC03, DQARC, QARC01, DQARC, DARC02, DSXTARC , SXTARCL01, \& DSXTARC , DARC04, DBXARC , BXARCL , DBXARC , DARC01, BXYC , DARC02, BPM , DARC03 , \& DQARC, QARC02, DQARC, DARC02, DSXTARC , SXTARCLO2, DSXTARC , DARC04, DBXARC ,

BXARCL , \& DBXARC , DARC01, BXYC , DARC02, BPM , DARC03 , DQARC , QARC01, DQARC , DARC02 , \& DSXTARC , SXTARCL01, DSXTARC, DARC04, DBXARC, BXARCL , DBXARC, DARC01, BXYC, DARCO2 \& BPM , DARC03, DQARC, QARC02, DQARC, DARC02, DSXTARC, SXTARCLO2, DSXTARC, DARCO4 \& DBXARC, BXARCL , DBXARC, DARC01, BXYC, DARC02, BPM, DARC03, DQARC, QARC0 1 , \& DQARC , DARC02, DSXTARC, SXTARCL01, DSXTARC, DARC04, DBXARC, BXARCL, DBXARC , DARC0 1 , \&

BXYC, DARC02, BPM , DARC03, DQARC, QARC02, DQARC, DARC02, DSXTARC, SXTARCLO2 , \& DSXTARC , DARC04, DBXARC , BXARCL , DBXARC , DARC01, BXYC , DARC02, BPM , DARC03 , \& DQARC, QARC01, DQARC, DARC02, DSXTARC, SXTARCL01, DSXTARC , DARC04, DBXARC, BXARCL , \&

DBXARC , DARC01, BXYC, DARC02, BPM , DARC03, DQARC, QARC02, DQARC , DARC02 , \& DSXTARC , SXTARCL02, DSXTARC, DARC04, DBXARC, BXARCL, DBXARC, DARC01, BXYC , DARCO2, \& BPM , DARC03, DQARC , QARC01, DQARC, DARC02, DSXTARC , SXTARCL01, DSXTARC, DARC04, \& DBXARC , BXARCL , DBXARC, DARC01, BXYC, DARC02, BPM, DARC03, DQARC, QARC02 , \& DQARC, DARC02, DSXTARC, SXTARCLO2, DSXTARC, DARC04, DBXARC, BXARCL, DBXARC , DARC0 1 , \& BXYC , DARC02 , BPM , DARC03, DQARC , QARC01, DQARC , DARC02, DSXTARC , SXTARCL01, \& DSXTARC , DARC04, DBXARC , BXARCL , DBXARC , DARC01, BXYC , DARC02, BPM , DARC03 , \& DQARC, QARC02, DQARC, DARC02, DSXTARC, SXTARCLO2, DSXTARC, DARC04, DBXARC , BXARCL , \& DBXARC , DARC01, BXYC , DARC02, BPM , DARC03, DQARC , QARC01, DQARC , DARC02 , \& DSXTARC, SXTARCL01, DSXTARC, DARC04, DBXARC, BXARCL, DBXARC, DARC01, BXYC, DARCO2, \& BPM , DARC03, DQARC, QARC02, DQARC, DARC02, DSXTARC, SXTARCLO2, DSXTARC, DARC04, \& DBXARC , BXARCL , DBXARC, DARC01, BXYC , DARC02, BPM , DARC03, DQARC, QARC01, \& DQARC, DARC02, DSXTARC, SXTARCL01, DSXTARC, DARC04, DBXARC, BXARCL, DBXARC , DARC0 1 , \& BXYC, DARC02, BPM , DARC03, DQARC, QARC02, DQARC, DARC02, DARC07, DARC04, \& 
DBXARC , BXARCL , DBXARC, DARC01, BXYC , DARC02, BPM , DARC03, DQARC, QARC01, \& DQARC , DARC02, DARC07, DARC04, DBXARC , BXARCL , DBXARC , DARC01, BXYC, DARC02, \& BPM , DARC03, DQARC, QARC02, DQARC, DARC02, DARC07, DARC04, DBXARC, BXARCL \& DBXARC , DARC01, BXYC , DARC02, BPM , DARC03, DQARC , QARC01, DQARC , DARC02 , \& DARC07, DARC04, DBXARC, BXARCL, DBXARC, DARC01, BXYC, DARC02, BPM, DARC0 3 \& DQARC, QARC02, DQARC , DARC02, DARC07, DARC04, DBXARC, BXARCL , DBXARC , DARC01, \& BXYC , DARC02, BPM , DARC03, DQARC, QARC01, DQARC , DARC02, DARC07, DARC04, \& DBXARC, BXARCL , DBXARC , DARC01, BXYC , DARC02 , BPM , DARC03, DQARC , QARC02 , \& DQARC, DARC02, DARC07, DARC04, DBXARC, BXARCL, DBXARC, DARC01, BXYC, DARCO2 , \& BPM , DARC03, DQARC , QARC01, DQARC , DARC02, DARC07, DARC04, DBXARC , BXARCL , \& DBXARC , DARC01, BXYC , DARC02, BPM , DARC03, DQARC, QARC02, DQARC , DARC02 , \& DARC07, DARC04, DBXARC, BXARCL , DBXARC, DARC01, BXYC, DARC02, BPM , DARC03 , \& DQARC, QARC01_H , MARCM , QARC01_H, DQARC, DARC02, DARC07, DARC04, DBXARC , BXARCL , \&

DBXARC , DARC01, BXYC, DARC02, BPM , DARC03, DQARC, QARC02, DQARC , DARC02 , \& DARC07, DARC04, DBXARC, BXARCL , DBXARC , DARC01, BXYC , DARC02, BPM , DARC03 , \& DQARC, QARC01, DQARC, DARC02, DARC07, DARC04, DBXARC, BXARCL , DBXARC, DARC01, \& BXYC , DARC02, BPM , DARC03 , DQARC , QARC02, DQARC , DARC02 , DARC07, DARC0 4 , \& DBXARC, BXARCL , DBXARC, DARC01, BXYC, DARC02, BPM, DARC03, DQARC, QARC01, \& DQARC , DARC02, DARC07, DARC04, DBXARC , BXARCL , DBXARC , DARC01, BXYC , DARC02, \& BPM , DARC03, DQARC, QARC02, DQARC, DARC02, DARC07, DARC04, DBXARC, BXARCL , \& DBXARC, DARC01, BXYC , DARC02, BPM , DARC03, DQARC, QARC01, DQARC, DARC02 , \& DARC07, DARC04, DBXARC, BXARCL, DBXARC, DARC01, BXYC, DARC02, BPM, DARC03 , \& DQARC, QARC02, DQARC , DARC02, DARC07, DARC04, DBXARC, BXARCL , DBXARC, DARC01, \& BXYC , DARC02 , BPM , DARC03 , DQARC , QARC01, DQARC , DARC02, DARC07, DARC04 , \& DBXARC , BXARCL , DBXARC, DARC01, BXYC, DARC02, BPM , DARC03, DQARC, QARC02 , \& DQARC, DARC02, DSXTARC, SXTARCLO2, DSXTARC, DARCO4, DBXARC, BXARCL, DBXARC , DARCO 1 , \& BXYC , DARC02, BPM , DARC03, DQARC, QARC01, DQARC, DARC02, DSXTARC, SXTARCL01, \& DSXTARC, DARC04, DBXARC, BXARCL , DBXARC, DARC01, BXYC , DARC02, BPM , DARC03, \& DQARC, QARC02, DQARC, DARC02, DSXTARC, SXTARCLO2, DSXTARC , DARC04, DBXARC, BXARCL , \& DBXARC , DARC01, BXYC , DARC02 , BPM , DARC03, DQARC , QARC01, DQARC , DARC02 , \& DSXTARC, SXTARCL01, DSXTARC, DARC04, DBXARC, BXARCL, DBXARC, DARC01, BXYC, DARCO2, \&

BPM, DARC03, DQARC, QARC02, DQARC, DARC02, DSXTARC, SXTARCL02, DSXTARC, DARC04, \& DBXARC, BXARCL , DBXARC, DARC01, BXYC, DARC02, BPM , DARC03, DQARC, QARC01, \& DQARC, DARC02, DSXTARC, SXTARCL01, DSXTARC, DARC04, DBXARC, BXARCL, DBXARC , DARC0 1 , \& BXYC, DARC02, BPM, DARC03, DQARC, QARC02, DQARC, DARC02, DSXTARC, SXTARCLO2 , \& DSXTARC, DARC04, DBXARC, BXARCL, DBXARC, DARC01, BXYC, DARC02, BPM, DARC03, \& 
DQARC, QARC01, DQARC, DARC02, DSXTARC, SXTARCL01, DSXTARC, DARC04, DBXARC , BXARCL , \&

DBXARC, DARC01, BXYC , DARC02, BPM , DARC03, DQARC, QARC02, DQARC, DARC02, \& DSXTARC, SXTARCL02, DSXTARC, DARC04, DBXARC, BXARCL, DBXARC, DARC01, BXYC ,

$\mathrm{DARCO} 2, \&$

BPM , DARC03, DQARC, QARC01, DQARC, DARC02, DSXTARC, SXTARCL01, DSXTARC, DARC04, \& DBXARC, BXARCL , DBXARC, DARC01, BXYC , DARC02, BPM , DARC03, DQARC, QARC02, \& DQARC, DARC02, DSXTARC, SXTARCLO2, DSXTARC, DARC04, DBXARC, BXARCL, DBXARC, DARC01, \& BXYC, DARC02, BPM , DARC03, DQARC, QARC01, DQARC, DARC02, DSXTARC, SXTARCL01, \& DSXTARC , DARC04, DBXARC, BXARCL , DBXARC, DARC01, BXYC, DARC02, BPM , DARC03, \& DQARC, QARC02, DQARC, DARC02, DSXTARC , SXTARCL02, DSXTARC, DARC04, DBXARC , BXARCL , \& DBXARC , DARC01, BXYC , DARC02, BPM , DARC03, DQARC, QARC01, DQARC, DARC02, \& DSXTARC, SXTARCL01, DSXTARC, DARC04, DBXARC, BXARCL , DBXARC , DARC01, BXYC , DARCO $2, \&$ BPM , DARC03, DQARC, QARC02, DQARC, DARC02, DSXTARC, SXTARCL02, DSXTARC, DARC04, \& DBXARC, BXARCL , DBXARC, DARC01, BXYC, DARC02, BPM , DARC03, DQARC, QARC01, \& DQARC, DARC02, DSXTARC, SXTARCL01, DSXTARC, DARC04, DBXARC, BXARCL, DBXARC, DARC0 $1, \&$

BXYC , DARC02, BPM , DARC03, DQARC, QARC02, DQARC, DARC02, DSXTARC, SXTARCL02, \& DSXTARC, DARC04, DBXARC, BXARCL , DBXARC, DARC01, BXYC, DARC02, BPM , DARC03, \& DQARC, QARC01, DQARC, DARC02, DSXTARC , SXTARCL01, DSXTARC, DARC04, DBXARC , BXARCL , \& DBXARC , DARC01, BXYC , DARC02, BPM , DARC03, DQARC, QARC02, DQARC, DARC02, \& DSXTARC, SXTARCL02, DSXTARC, DARC04, DBXARC , BXARCL , DBXARC , DARC01, BXYC , DARCO $2, \&$ BPM , DARC03, DQARC, QARC01, DQARC, DARC02, DSXTARC, SXTARCL01, DSXTARC, DARC04, \& DBXARC, BXARCL , DBXARC, DARC01, BXYC, DARC02, BPM , DARC03, DQARC, QARC02, \& DQARC, DARC02, DSXTARC, SXTARCL02, DSXTARC, DARC04, DBXARC, BXARCL, DBXARC , $\mathrm{DARC} 01, \&$ BXYC , DARC02, BPM , DARC03, DQARC, QARC01, DQARC, DARC02, DSXTARC, SXTARCL01, \& DSXTARC , DARC04, DBXARC, BXARCL , DBXARC, DARC01, BXYC, DARC02, BPM , DARC03, \& DQARC, QARC02, DQARC, DARC02, DSXTARC, SXTARCLO2, DSXTARC, DARC04, DBXARC , BXARCL , \& DBXARC , DARC01, BXYC , DARC02, BPM , DARC03, DQARC, QARC01, DQARC, DARC02, \& DSXTARC, SXTARCL01, DSXTARC, DARC04, DBXARC, BXARCL , DBXARC, DARC01, BXYC , DARC02, \& BPM , DARC03, DQARC , QARC02, DQARC, DARC02, DSXTARC, SXTARCL02, DSXTARC, DARC04, \& DBXARC, BXARCL , DBXARC, DARC01, BXYC , DARC02, BPM , DARC03, DQARC, QARC01,\& 
DQARC, DARC02, DSXTARC, SXTARCL01, DSXTARC, DARC04, DBXARC, BXARCL , DBXARC , DARCO 1 , \&

BXYC, DARC02, BPM , DARC03, DQARC, QARC02, DQARC, DARC02, DSXTARC , SXTARCLO2 , \& DSXTARC , DARC04, DBXARC , BXARCL , DBXARC , DARC01, BXYC , DARC02, BPM , DARC03 \& \& DQARC, QARC01, DQARC, DARC02, DSXTARC , SXTARCL01, DSXTARC , DARC04, DBXARC , BXARCL , \&

DBXARC, DARC01, BXYC, DARC02, BPM , DARC03, DQARC, QARC02, DQARC, DARC02 , \& DSXTARC, SXTARCLO2, DSXTARC, DARC04, DBXARC, BXARCL, DBXARC, DARC01, BXYC,

DARCO2, \&

BPM, DARC03, DQARC, QARC01, DQARC, DARC02, DSXTARC, SXTARCL01, DSXTARC, DARC04, \& DBXARC , BXARCL , DBXARC, DARC01, BXYC, DARC02, BPM, DARC03, DQARC, QARCO2 , \& DQARC, DARC02, DSXTARC, SXTARCLO2, DSXTARC, DARC04, DBXARC , BXARCL , DBXARC, DARCO $1, \&$ BXYC, DARC02, BPM, DARC03, DQARC, QARC01, DQARC, DARC02, DSXTARC, SXTARCL01, \& DSXTARC , DARC04, DBXARC, BXARCL , DBXARC, DARC01, BXYC , DARC02, BPM , DARC03 , \& DQARC, QARC02, DQARC, DARC02, DSXTARC, SXTARCLO2, DSXTARC, DARC04, DBXARC, BXARCL , \& DBXARC , DARC01, BXYC , DARC02, BPM , DARC03, DQARC , QARC01, DQARC , DARC02 , \& DSXTARC, SXTARCL01, DSXTARC, DARC04, DBXARC, BXARCL, DBXARC, DARC01, BXYC, DARCO2, \&

BPM, DARC03, DQARC, QARC02, DQARC, DARC02, DSXTARC, SXTARCL02, DSXTARC, DARC04, \& DBXARC , BXARCL , DBXARC, DARC01, BXYC , DARC02 , BPM , DARC03, DQARC , QARC01 , \& DQARC , DARC02, DSXTARC, SXTARCL01, DSXTARC, DARC04, DBXARC, BXARCL, DBXARC , DARCO 1 , \&

BXYC, DARC02, BPM, DARC03, DQARC, QARC02, DQARC, DARC02, DSXTARC, SXTARCLO2, \& DSXTARC, DARC04, DBXARC, BXARCL , DBXARC, DARC01, BXYC, DARC02, BPM , DARC03, \& DQARC, QARC01, DQARC, DARC02, DSXTARC, SXTARCL01, DSXTARC, DARC04, DBXARC , BXARCL , \& DBXARC , DARC01, BXYC , DARC02 , BPM , DARC03, DQARC , QARC02 , DQARC , DARC02 , \& DSXTARC, SXTARCLO2, DSXTARC, DARC04, DBXARC, BXARCL, DBXARC, DARC01, BXYC, DARCO2, \&

BPM , DARC03, DQARC, QARC01, DQARC, DARC02, DSXTARC, SXTARCL01, DSXTARC, DARC04, \& DBXARC , BXARCL , DBXARC , DARC01, BXYC , DARC02, BPM , DARC03, DQARC, QARC02 , \& DQARC , DARC02, DARC07, DARC04, DBXARC, BXARCL, DBXARC, DARC01, BXYC, DARC02, \& BPM , DARC03, DQDS , QDS01, DQDS , DARC02, DARC07, DARC04, DBXDS , BXDS1L , \& DBXDS , DARC01, BXYC , DARC02, BPM , DARC03 , DQDS , QDS02, DQDS , DARC02 , \& DARC07, DARC0 4, DBXDS , BXDS1L, DBXDS , DARC01, BXYC , DARC02 , BPM , DARC0 3 , \& DQDS , QDS03 , DQDS , DARC02 , DARC07 , DARC04, DBXDS , BXDS2L , DBXDS , DARC01 \& BXYC, DARC02, BPM , DARC03, DQDS, QDS04, DQDS, DARC02, DARC07, DARC04, \& DBXDS , BXDS2L, DBXDS, DARC06, DSB22DS00, DQSB22DS, QSB22DSO4, DQSB22DS , DSB22DSO0, BPM , \& 


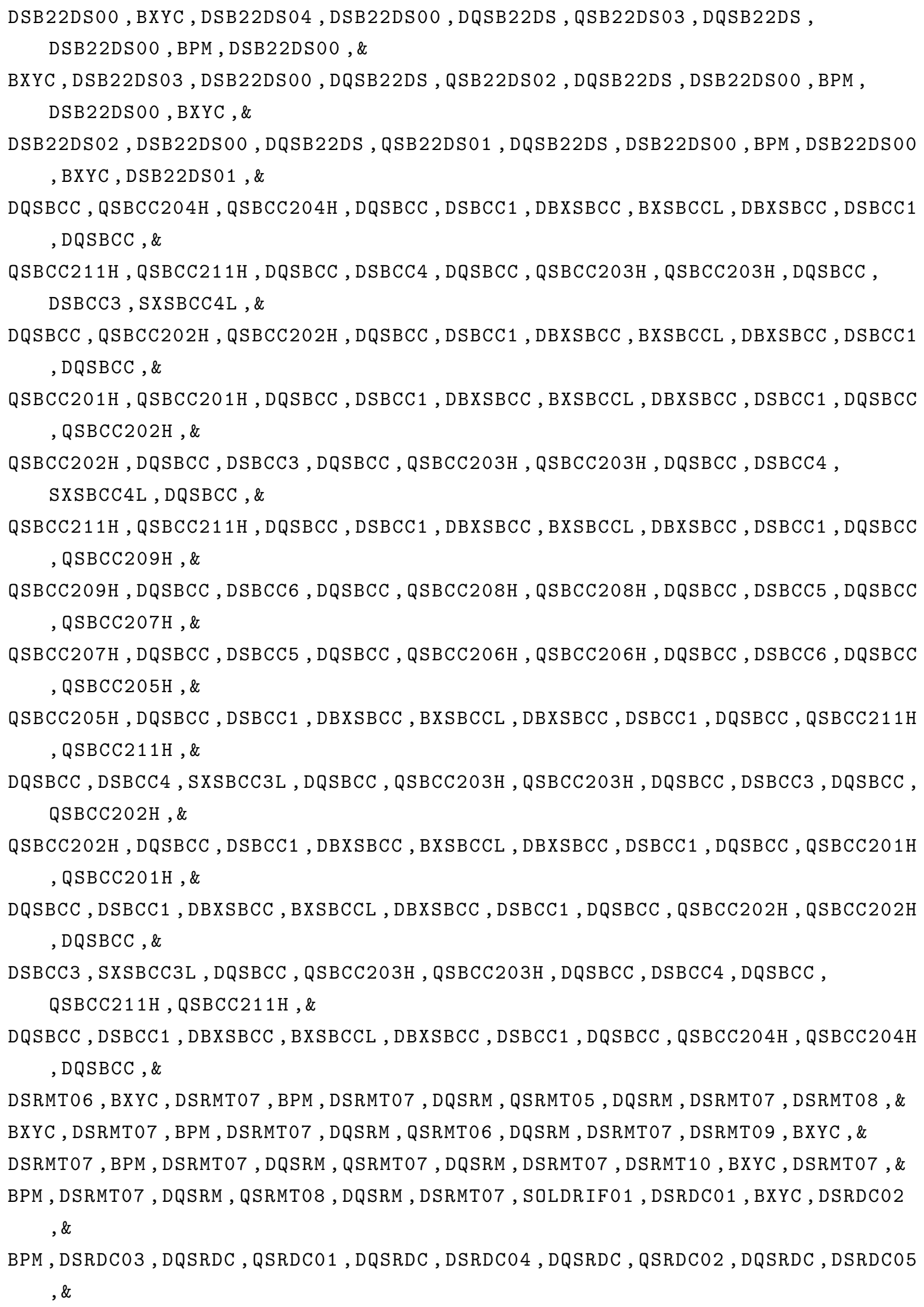


BXYC , DSRDC0 2 , BPM , DSRDC0 3, DQSRDC , QSRDC03, DQSRDC , DSRDC0 3, BXYC , DSRDC0 2 \& BPM , DSRDC05, DQSRDC , QSRDC02, DQSRDC , DSRDC04, DQSRDC , QSRDC01, DQSRDC , DSRDC03 , \& BXYC , DSRDC02, BPM , DSRDC01, SOLDRIF01, DSRM201, BPM, DSRM202, BXYC , DSRM202 , \& DQSRM2 , QSRM205, DQSRM2 , DSRM203, BPM , DSRM202, BXYC , DSRM202, DQSRM2 , QSRM206 , \& DQSRM2 , DSRM203, DBXSR , BXSR1L , DBXSR , DSRM204, BPM , DSRM202 , BXYC , DSRM202 , \& DQSRM2 , QSRM207, DQSRM2 , DSRM204, DQSRM2 , QSRM208_H , MSRMT2 , QSRM208_H , DQSRM2 , DSRM204, \&

DQSRM2 , QSRM207, DQSRM2 , DSRM202 , BXYC , DSRM202, BPM , DSRM204 , DBXSR , BXSR1L , \& DBXSR , DSRM203, DQSRM2, QSRM206 , DQSRM2 , DSRM202, BXYC , DSRM202, BPM , DSRM203 , \& DQSRM2 , QSRM205 , DQSRM2 , DSRM202, BXYC , DSRM202 , BPM , DSRM201, SOLDRIF02 , DSRDC01, \&

BXYC , DSRDC02 , BPM , DSRDC0 3 , DQSRDC , QSRDC01, DQSRDC , DSRDC04, DQSRDC , QSRDC02 \& DQSRDC , DSRDC05, BXYC , DSRDC02, BPM , DSRDC03, DQSRDC , QSRDC03, DQSRDC, DSRDC03 \& \& BXYC , DSRDC0 2 , BPM , DSRDC05, DQSRDC , QSRDC02, DQSRDC , DSRDC04, DQSRDC, QSRDC0 1 , \& DQSRDC , DSRDC03, BXYC, DSRDC02, BPM, DSRDC01, SOLDRIF02, DSRM301, BPM, DSRM302, \& BXYC , DSRM302, DQSRM3 , QSRM304, DQSRM3 , DSRM303, DBXSR , BXSR2L, DBXSR, DSRM304, \& BPM , DSRM302 , BXYC , DSRM302, DQSRM3 , QSRM305, DQSRM3 , DSRM304 , DQSRM3 , QSRM306_H , \&

MSRMT3 , QSRM306_H , DQSRM3 , DSRM304, DQSRM3 , QSRM305 , DQSRM3 , DSRM302 , BXYC , DSRM302, \&

BPM , DSRM304, DBXSR , BXSR2L , DBXSR, DSRM303, DQSRM3 , QSRM304, DQSRM3 , DSRM302, \&

BXYC , DSRM302, BPM , DSRM301, DAEL2SF1, BXYC, DAEL2SFO, BPM , DAEL2SFO, DQAEL2SF , \& QAEL2SF1, DQAEL2SF, DAEL2SF2, BXYC, DAEL2SFO, BPM , DAEL2SFO, DQAEL2SF, QAEL2SF2 , DQAEL2SF , \&

DAEL2SF 3, BXYC , DAEL2SFO, BPM, DAEL2SFO, DQAEL2SF, QAEL2SF3, DQAEL2SF, DAEL2SF 4 , BXYC, \&

DAEL2SF0, BPM, DAEL2SF0, DQAEL2SF, QAEL2SF4, DQAEL2SF, DAEL2SF5, BXYC, DAEL2SFO , BPM , \&

DAEL2SF0, DQAEL2SF, QAEL2SF5, DQAEL2SF, DAEL2SF6, DSF1_US, BXYC, DSF0_US , BPM , DSFO_US , \&

DQSF_US, QSF1_US, DQSF_US , DSF1_US , BXYC , DSFO_US, BPM , DSFO_US, DQSF_US , QSF2_US , \&

DQSF_US, DSF1_US, BXYC, DSFO_US, BPM, DSFO_US , DQSF_US , QSF1_US, DQSF_US , DSF1_US , \&

BXYC , DSFO_US , BPM , DSFO_US , DQSF_US, QSF2_US, DQSF_US , DSF1_US , BXYC, DSFO_US , \& BPM , DSFO_US , DQSF_US , QSF1_US, DQSF_US , DSF1_US , BXYC, DSFO_US , BPM, DSFO_US , \& DQSF_US , QSF2_US, DQSF_US , DSF1_US , BXYC, DSFO_US , BPM, DSFO_US , DQSF_US , QSF1_US , \&

DQSF_US , DSF1_US, BXYC, DSFO_US , BPM, DSFO_US, DQSF_US , QSF2_US, DQSF_US , DSF1_US , \& 
BXYC , DSF0_US, BPM, DSF0_US, DQSF_US, QSF1_US, DQSF_US, DSF1_US, BXYC, DSF0_US , \& BPM , DSFO_US , DQSF_US , QSF2_US , DQSF_US , DSF1_US , BXYC , DSFO_US , BPM , DSFO_US , \& DQSF_US, QSF1_US_H)

USE , CR 4

RETURN 


\section{VITA}

Salvador Isaac Sosa Güitrón

Department of Physics

Old Dominion University

Norfolk, VA 23529

\section{Education}

Old Dominion University, Norfolk, Va.

- PhD Candidate in Accelerator Physics, 2014-Present.

- Master of Science, 2014.

Benemérita Universidad Autónoma de Puebla, Puebla, Mexico.

- Bachelor of Science in Physics, 2012.

\section{Research Experience}

Center for Accelerator Science, Old Dominion University, Norfolk, Va.

- 2016-Present: Graduate research assistant simulating the dynamics of JLEIC beams with local bunch crabbing scheme in the interaction region.

- 2014-2016: Graduate research assistant measuring the surface resistance of superconducting films for accelerator applications at the SRF Institute in Jefferson Lab.

Typeset using $\mathrm{AT}_{\mathrm{EX}}$. 